\title{
Molecular studies of FLCN-related diseases
}

Citation for published version (APA):

Luijten, M. N. H. (2014). Molecular studies of FLCN-related diseases. [Doctoral Thesis, Maastricht University]. Universitaire Pers Maastricht. https://doi.org/10.26481/dis.20141212ml

Document status and date:

Published: 01/01/2014

DOI:

10.26481/dis.20141212ml

Document Version:

Publisher's PDF, also known as Version of record

\section{Please check the document version of this publication:}

- A submitted manuscript is the version of the article upon submission and before peer-review. There can be important differences between the submitted version and the official published version of record.

People interested in the research are advised to contact the author for the final version of the publication, or visit the DOI to the publisher's website.

- The final author version and the galley proof are versions of the publication after peer review.

- The final published version features the final layout of the paper including the volume, issue and page numbers.

Link to publication

\footnotetext{
General rights rights.

- You may freely distribute the URL identifying the publication in the public portal. please follow below link for the End User Agreement:

www.umlib.nl/taverne-license

Take down policy

If you believe that this document breaches copyright please contact us at:

repository@maastrichtuniversity.nl

providing details and we will investigate your claim.
}

Copyright and moral rights for the publications made accessible in the public portal are retained by the authors and/or other copyright owners and it is a condition of accessing publications that users recognise and abide by the legal requirements associated with these

- Users may download and print one copy of any publication from the public portal for the purpose of private study or research.

- You may not further distribute the material or use it for any profit-making activity or commercial gain

If the publication is distributed under the terms of Article $25 \mathrm{fa}$ of the Dutch Copyright Act, indicated by the "Taverne" license above, 


\section{Molecular studies of FLCN-related diseases}

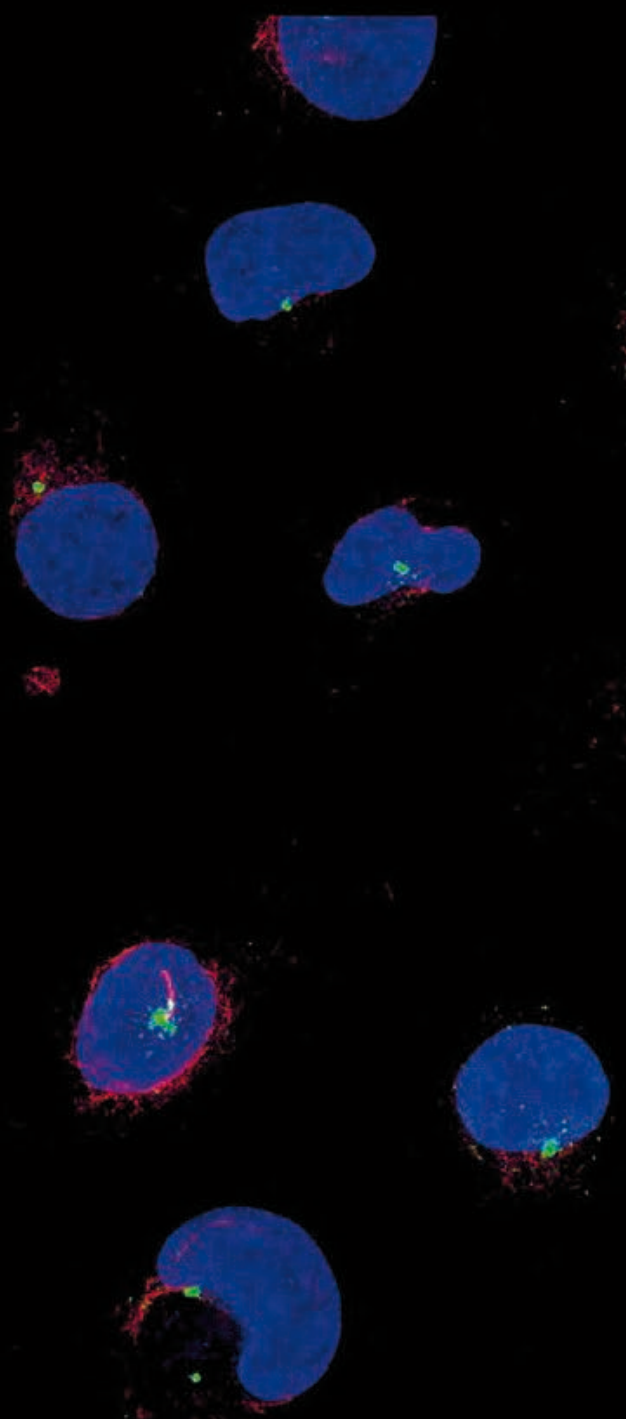

Monique N.H. Luijten
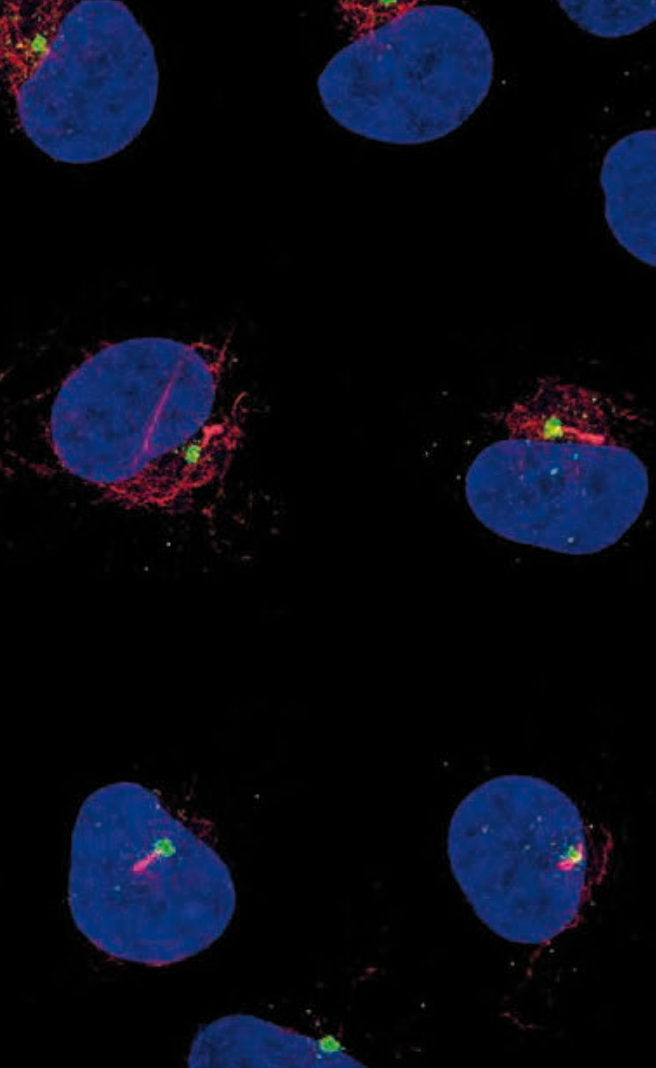


\section{Molecular studies of FLCN-related diseases}

Monique N.H. Luijten

ISBN 9789461593726

Cover design by Sarah Zwarts

Layout by Monique N.H. Luijten

Printed by Datawyse | Universitaire Pers Maastricht

Copyright (C) 2014 M.N.H. Luijten, Maastricht, The Netherlands

All rights reserved. No part of this thesis may be reproduced, stored or transmitted in any form or by any means without prior written permission of the author.

Cover illustration: Maximum projection of HK-2 cells immunofluorescently stained for acetylated alpha tubulin which labels cilia in red, pericentrin which labels the basal body in green and DAPI which labels DNA in blue. 


\title{
Molecular studies of FLCN-related diseases
}

\author{
PROEFSCHRIFT
}

ter verkrijging van de graad van doctor aan de Universiteit Maastricht op gezag van de Rector Magnificus, Prof. dr. L.L.G. Soete volgens het besluit van het College van Decanen in het openbaar te verdedigen op vrijdag 12 december 2014 om 10.00 uur

door

Monique Nicole Helena Luijten

Geboren op 26 november 1986 te Cadier en Keer

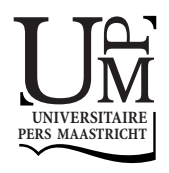




\section{Promotor}

Prof. dr. M.A.M. van Steensel

\section{Copromotores}

Dr. B.J. Coull

Prof. dr. F. Müller (University of Birmingham, United Kingdom)

\section{Beoordelingscommissie}

Prof. dr. F.C.S. Ramaekers (voorzitter)

Dr. F. van Eeden (University of Sheffield, United Kingdom)

Prof. dr. M. van Engeland

Prof. dr. V.V.A.M. Knoers (Universitair Medisch Centrum Utrecht)

Dr. J. Vanoevelen

This work was financially supported by KWF Kankerbestrijding, Stichting Sint Annadal, De Raad van Bestuur Maastricht UMC+, The Association for International Cancer Research and Myrovlytis Trust. 
Aan mijn ouders 



\section{Contents}

$\begin{array}{lll}\text { CHAPTER } 1 \quad \text { General introduction } & 9\end{array}$

CHAPTER 2 Birt-Hogg-Dubé syndrome is a novel ciliopathy 33

CHAPTER 3 The role of flcn in zebrafish embryonic development $\quad 71$

CHAPTER 4 Familial multiple discoid fibromas, a Birt-Hogg-Dubé 95 syndrome lookalike, is caused by a truncating FNIP1 mutation

$\begin{array}{lll}\text { CHAPTER } 5 \quad \text { General discussion } & 119\end{array}$

$\begin{array}{llr}\text { CHAPTER } 6 \quad \text { Valorisation } & 149\end{array}$

$\begin{array}{lll}\text { CHAPTER } 7 & \text { Summary } & 161\end{array}$

$\begin{array}{ll}\text { Samenvatting } & 166\end{array}$

$\begin{array}{ll}\text { Acknowledgements / Dankwoord } & 170\end{array}$

$\begin{array}{ll}\text { Curriculum vitae } & 172\end{array}$ 



\section{CHAPTER 1}

\section{General introduction}

Adapted from:

Claessens T, Vernooij M, Luijten M, Coull BJ, Van Steensel MAM. What's new in Birt-Hogg-Dubé syndrome? Expert Review of Dermatology. 2012 Dec;7(6):521-8.

Vernooij M, Claessens T, Luijten M, van Steensel MA, Coull BJ. Birt-Hogg-Dube syndrome and the skin. Familial cancer. 2013 Sep;12(3):381-5. 
CHAPTER 1 


\section{Birt-Hogg-Dubé syndrome clinical characteristics}

In 1975, Otto Paul Hornstein and Monika Knickenberg described two siblings with multiple perifollicular fibromas on the head and upper body as a distinct inherited condition (1). One of the two siblings was reported to have polyps of the colon (1). Two years later, the Canadian physicians Arthur R. Birt, Georgina R. Hogg and William J. Dubé presented a report of a new disorder characterised by development of skin fibrofolliculomas in association with medullary carcinoma of the thyroid (2), wherefrom Birt-Hogg-Dubé syndrome (BHD; OMIM \#135150) derived its name. In 1999 it became clear that there are no histological differences between the perifollicular fibromas described by Hornstein and Knickenberg and the fibrofolliculomas in BHD (3) and thus they are the same disease, which should more appropriately be named Hornstein-Birt-Hogg-Dubé syndrome (4).

Approximately $80 \%$ of BHD patients will develop fibrofolliculomas, which are asymptomatic, white or skin-coloured, dome-shaped papules on the skin (Figure 1A). They usually present during the third or fourth decade of life on the face, neck and/or upper torso $(2,5-8)$. The number of lesions can vary greatly according to the anatomical site in a single patient and from one individual to another $(5,7,9)$. Histologically, fibrofolliculomas are characterised by thin, elongated strands of epithelial cells extending from the hair follicle (2).
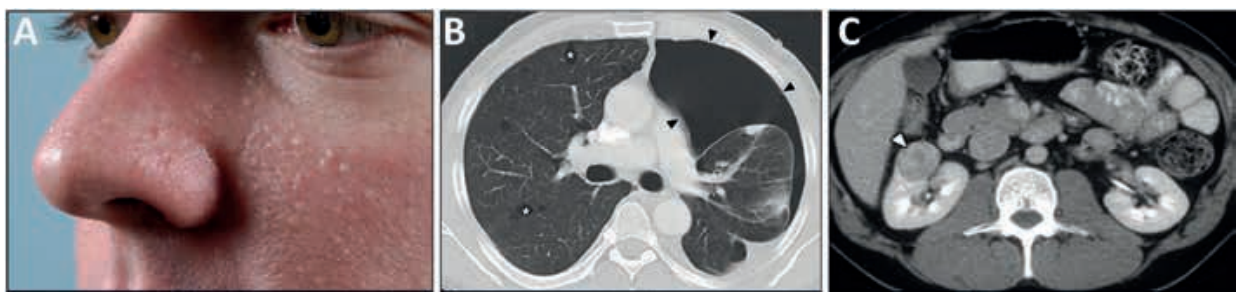

Figure 1 - The Birt-Hogg-Dubé syndrome phenotype

Patients with Birt-Hogg-Dubé syndrome have an increased risk of (A) fibrofolliculomas, (B) lung cysts (asterisks) and pneumothorax (arrowheads) and (C) renal cancer (arrowhead). Adapted from $(10,11)$ permission from Elsevier and AACR.

Although BHD was first characterised based on the skin phenotype, more than $80 \%$ of BHD patients develop multiple pulmonary cysts in the basal regions of the lung (Figure 1B) (5-8). These cysts are considered precursor lesions for spontaneous pneumothorax, leading to a 50-fold increased risk for BHD patients compared to their unaffected siblings (11). Patients have been described with one or recurrent pneumothoraxes at an age ranging from 7 to 46 years $(6-8,11)$.

The most threatening manifestation of BHD is the increased risk of renal cancer (Figure 1C), which is seven times higher in BHD patients compared to their unaffected siblings (11). The frequency of renal cancer among BHD-affected individuals ranges between $20-30 \%$ in different cohorts $(5,6,8)$. BHD-associated renal carcinomas are mainly of chromophobe or mixed chromophobe and 
oncocytic histological subtypes, however clear cell, papillary carcinoma and several mixed patterns can also occur (Figure 2) (12). Tumours are usually diagnosed at a relatively young age and can develop uni- or bilaterally (5). They rarely metastasise, but patients with metastasis have been described $(8,9,13,14)$.

Initially BHD was also considered to be associated with colorectal neoplasia (1). However, subsequent studies could not confirm a causal relationship between BHD and colon polyps or carcinomas $(7,8,11)$ and it has been suggested that this relationship only exists in specific families $(9,15)$. Association of BHD with various neoplasms, including parathyroid adenoma $(7,16)$, thyroid nodules $(7)$ and cancer $(2,6)$, parotid tumours $(6,17)$ and melanoma $(6,9)$ has been reported, but remains controversial.
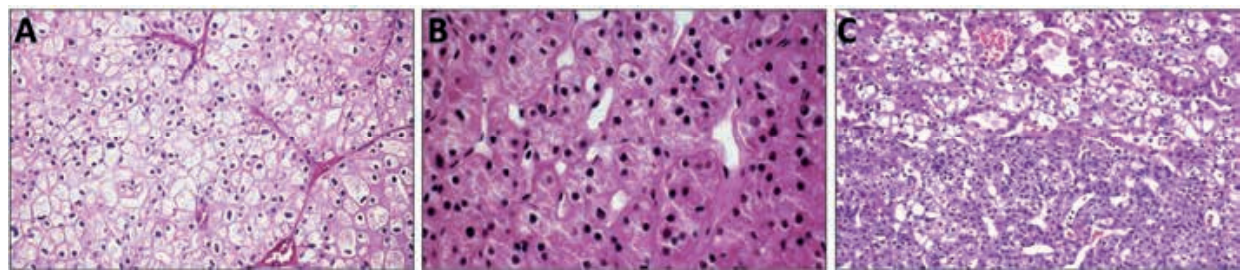

Figure 2 - Histopathology of BHD renal tumours

BHD renal tumours can be of varying histopathology, including (A) chromphobe renal carcinoma, (B) oncocytoma and (C) oncocytic hybrid. Adapted from (18) with permission from Elsevier.

\section{FLCN - The Birt-Hogg-Dubé syndrome causative gene}

BHD has autosomal dominant inheritance. In 2001, the BHD-associated gene locus was mapped to chromosome $17 \mathrm{p} 11.2(19,20)$ and subsequently the causative gene was identified as the FLCN (also known as BHD) gene, encoding the highly evolutionarily conserved protein folliculin (FLCN) (21). Mutations in FLCN are found along the entire length of the gene in BHD patients (Figure 3)(5). To date, over 100 unique pathogenic mutations have been identified and are documented in two FLCN mutation databases $(22,23)$. While the majority of FLCN mutations are either frameshift or nonsense, splice site and missense mutations have also been reported. Almost all mutations are predicted to prematurely truncate the FLCN protein $(5,21)$. In $53 \%$ of all BHD families an insertion or deletion has been found at the mononucleotide $(\mathrm{C})_{8}$ tract within exon 11 , making it a mutational hotspot $(5,9,21)$. Worldwide approximately 200 families have been clinically diagnosed with BHD and in approximately 85\% of these families a germline FLCN mutation has been identified $(5,6,8,10)$. As a result of its clinical heterogeneity, the disease is probably underdiagnosed. Not all FLCN mutation carriers develop fibrofolliculomas and patients have been reported with either renal neoplasia, or pneumothorax, or both $(5,6,9)$. The variability in phenotypic expression is also observed among patients from the same family, indicating that environmental 
exposure may contribute to the variability of phenotypic expression (5). To date, no convincing genotype-phenotype relationship has been determined for BHD (6).

Current therapeutic options for BHD patients are limited and can only treat symptoms, not the underlying cause. Fibrofolliculomas can be temporarily removed using surgery, cautery or laser therapy, but none of these techniques are curative $(24,25)$. Despite the presence of cysts, the BHD lung functions normally (26). Therefore, the lungs do not need treatment unless pneumothorax occurs, which is treated similarly to sporadic primary pneumothorax (10). Renal cancer is generally treated using nephron-sparing surgery to spare renal function and prevent metastasis, but after treatment the cancer can recur. Also, patients can develop several tumours during their lifetime, necessitating multiple surgeries. Conclusively, there is a need for BHD-specific treatment options and strategies to prevent renal cancers from developing. Therefore, it is crucial to unravel BHD pathophysiology and FLCN's function in cellular physiology.

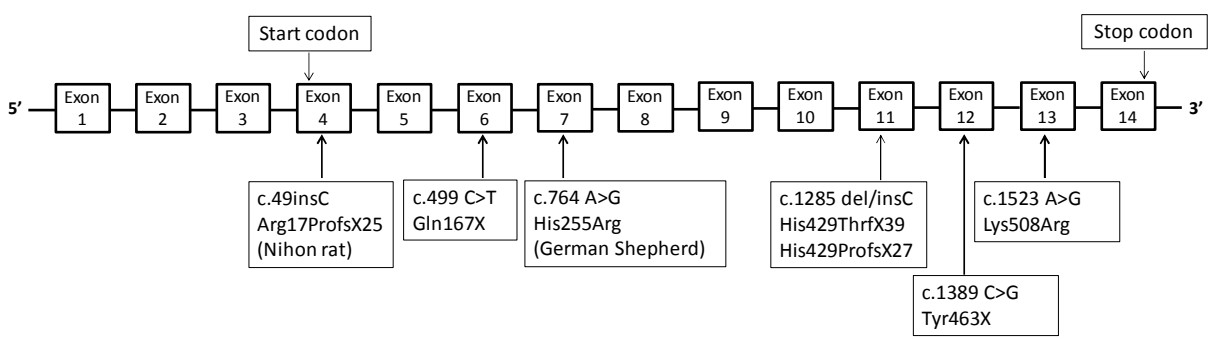

Figure 3 - Schematic of the FLCN gene with selected pathogenic mutations

A complete overview of all mutations can be found in the FLCN mutation databases $(22,23)$.

\section{FLCN as a tumour suppressor}

The exact function of FLCN is currently unknown, but the protein is highly conserved across species (Figure 4), suggesting an important biological role in a wide range of organisms. Genomic deletion of the Drosophila FLCN homolog $(D B H D)$ resulted in a nutrition-dependent growth delay in the larvae which could be partially rescued by human FLCN (27), indicating that DBHD and human FLCN are involved in common mechanisms.

FLCN is thought to act as a tumour suppressor, as it has been reported that BHD tumours have no expression of FLCN mRNA (28) and a high frequency of somatic second hit mutations (29). Two naturally occurring animal models for BHD have been described. One model is the Nihon rat (30), resulting from an insertion of a cytosine in the $\mathrm{C}_{5}$ tract of exon 3 , causing a frameshift and premature stop codon (31). The second model is in German Shepherd dogs (32), caused by a missense mutation in exon 7, resulting in FLCN-H225R on the protein level (33). In both models, homozygosity for the mutation is embryonically lethal. This was also observed for mouse knockouts and suggests that FLCN has a vital role in 
embryogenesis (34-38). Heterozygous animals develop bilateral, multiple kidney cysts, which further develop into renal tumours. Both canine and murine tumours show either loss of heterozygosity or somatic second hit mutations $(31,39)$, supporting the Knudson two hit model that postulates both wild-type alleles of a tumour suppressor gene must be inactivated prior to tumour growth (40). Furthermore, injection of the UOK257 cell line (considered to be FLCN-null (41)) derived from a BHD renal carcinoma into nude mice induced tumour development. This tumour cell growth was suppressed by re-expression of FLCN (42).

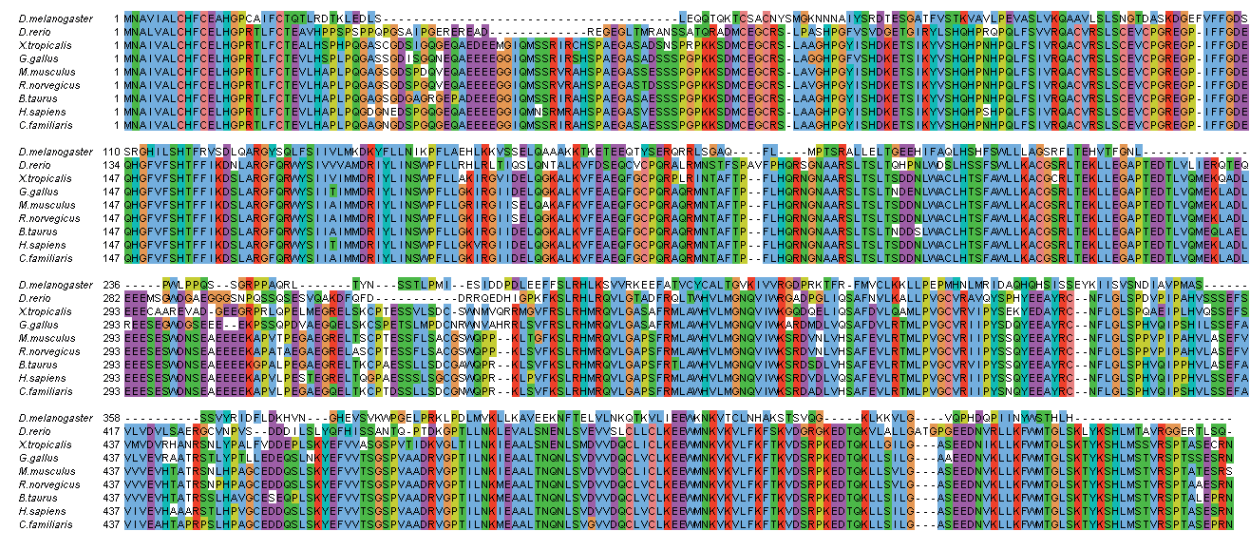

Figure 4 - FLCN conservation across species

Alignment of FLCN protein sequence of indicated species made using Clustal Omega $(43,44)$, manually edited using Jalview (45) and displayed with Clustal X colour scheme.

\section{FLCN protein function: link with mTOR signalling}

The BHD phenotype is similar to that of Von-Hippel Lindau disease (VHL; OMIM \#193300, caused by mutation in pVHL). In addition, BHD is a member of the hamartoma syndrome family, which includes Cowden syndrome (OMIM \#158350, PTEN), Peutz-Jeghers syndrome (OMIM \#175200, LKB1) and tuberous sclerosis complex (TSC; OMIM \#191100, hamartin, tuberin). These syndromes are all dominantly inherited and predispose to a variety of benign and malignant growths in multiple organ systems, including the kidney. The hamartoma syndromes have been reported to show mTOR deregulation (46). As a result, many groups have looked at the FLCN-mTOR relationship, which seems to be complex and context dependent.

Rodent models for BHD have been extensively characterised, but have yielded conflicting data on the consequences of FLCN mutations for mTOR regulation. Conditional, kidney-specific knockout of the Flcn gene in mice at two independent occasions resulted in the rapid development of enlarged polycystic kidneys and death from renal failure by 3 weeks of age $(37,38)$. Kidney lesions were characterised by the activation of Raf-Erk1/2 and Akt-mTOR pathways, increased 
expression of cell cycle proteins and cell proliferation $(37,38)$. Rapamycin (an inhibitor of mTOR) was able to mitigate the phenotype. From these data and in vitro work with BHD-derived tumour cells, it was concluded that FLCN has a role in regulating mTOR, probably downstream of AMPK (47). However, Hudon et al. examined another kidney-specific knockout model and determined that loss of FLCN expression leads to context-dependent effects on mTOR activation, as measured by $\mathrm{S} 6$ phosphorylation (48). Indeed, solid tumours and normal kidneys show decreased phospho-S6 upon diminished FLCN expression. In FLCN-negative renal cysts, phospho-S6 was either elevated or absent. In support of Hudon's findings, Hartman et al. reported mice heterozygous for a truncating Flcn mutation and which developed kidney tumours that did not show significant mTOR activation (35). Using fission yeast (Schizosaccharomyces pombe) as their model, Slegtenhorst et al. showed that the $S$. pombe FLCN ortholog activated Tor2 (yeast TOR), thus having an opposite effect to the TSC proteins (49). This finding seems to run counter to expectations. Slegtenhorst et al. proposed a model in which $S$. pombe Bhd regulates Tor 2 separately from the Tsc proteins. It does not explain why in humans both mTOR activation and lack thereof could cause similar phenotypes. Finally, we examined a BHD patient kidney tumour and did not find any indications of mTOR activation (50). Treatment of BHD fibrofolliculomas with topical rapamycin did not result in cosmetic improvement (51). Taken together these data suggest that mTOR deregulation is not a major determinant of the BHD phenotype and that knockout mice are probably not the best tools for studying BHD pathophysiology.

\section{The FLCN protein functions in various signalling pathways}

In 2006, the first direct interactor of FLCN was identified and named folliculininteracting protein 1 (FNIP1) (47). FNIP1's function is currently unknown, but it has been shown to interact with 5'-AMP activated protein kinase (AMPK) (47), which is a regulator of cellular energy homeostasis (52). In 2008, two groups identified a second novel binding partner for FLCN, FNIP1-like or Folliculin interacting protein 2 (FNIP2/FNIPL/MAP01), which showed 49\% identity to FNIP1 $(53,54)$. FNIP2 was found to form protein complexes with FLCN, AMPK and FNIP1 and is involved in $0^{6}$-methylguanine-induced apoptosis (55). FLCN also interacts with plakophilin-4/p0071, a component of the desmosomal and adherens junctions, which modulate cell adhesion $(56,57)$. Using Drosophila melanogaster as their model, Gaur et al. determined an interaction between dFLCN and the 19S proteasomal ATPase Rpt4, through which FLCN negatively regulates rRNA synthesis (58). Additionally, FLCN has been linked to JAK-STAT signalling as the Drosophila melanogaster FLCN ortholog functions downstream or in parallel with the JAK-STAT pathway to regulate male germline stem cell differentiation (59). In mammalian FLCN-null cells the kinase Jak1 was shown to be downregulated (42). 


\section{CHAPTER 1}

The Caenorhabditis elegans FLCN ortholog F22D3.2 regulates longevity in a hypoxia-inducible factor-1 (hif-1)-dependent manner (60). In addition, it was shown in vitro that FLCN deficiency results in increased HIF transcriptional activity (61). HIF transcription factors are known to be involved in sporadic renal cancer (62). It was determined that known downstream targets of both HIF1 $\alpha$ and HIF2 $\alpha$, including VEGF, BNIP3, CCND1 and GLUT1, were also upregulated. In addition, cells lacking FLCN have lost metabolic flexibility and favour glycolytic rather than lipid metabolism (the 'Warburg effect'). BHD renal tumour cells utilised L-lactate as a metabolic fuel. As a result of their higher dependency on glycolysis, growth of these cells was selectively inhibited by treatment with 2-deoxyglucose (61). Perhaps glycolytic metabolism constitutes a potential therapeutic target in BHD. Certainly, it seems that FLCN is involved in the regulation of cellular metabolism as increased HIF activity was previously reported to negatively regulate mitochondrial biogenesis (63). In contrast, Klomp et al. used gene expression profiling to show activation of PGC1 $\alpha$-mediated signalling in BHD-derived renal tumours (64). PGC1 $\alpha$ regulates mitochondrial biogenesis, and indeed the tumours examined contained more mitochondria than normal kidney tissue. It is not clear to what extent this phenomenon is specific to BHD, as increased numbers of mitochondria have been previously observed in various types of kidney cancer (65).

Additionally, two groups independently reported an interaction with TGFß signalling. Hong et al. found decreased expression of several TGFß pathway genes, including TGFß2 and activin A (66). The latter protein suppressed anchorageindependent growth of FLCN-deficient UOK257 (41) renal cancer cells, whereas TGFß2, curiously, seemed to increase it. Hong et al. concluded that suppression of TGFß-signalling contributes to malignancy in BHD. While this report places FLCN upstream of TGFß, Cash et al. found an effect of BHD downstream of TGFßsignalling, on canonical Smad target gene promoters (67). They did not find any effects on the signalling components themselves, contrary to what Hong et al. reported. Cash et al. suggest that the discrepancy might be due to the fact that they used BHD-null embryonic stem cells rather than UOK257. Apparently, cell type matters a great deal when studying FLCN's functions. However, we noted that transcription factor E3 (TFE3) collaborates with Smad proteins in regulating the transcription of various TGFß-induced genes such as PAI-1 (68). Hong et al. demonstrated that loss of FLCN causes increased nuclear accumulation of the latter transcription factor, which has been previously implicated in renal carcinogenesis (69). These data were confirmed by Betschinger et al., who showed that the exclusion of TFE3 from the nucleus by FLCN is necessary for embryonic stem cells to exit naive pluripotency (70). FLCN was also shown to regulate nuclear localisation of transcription factor EB (TFEB), closely related to TFE3, via phosphorylation of TFEB serine 211 (71). Thus, FLCN appears to act as a negative regulator of transcription for a number of potentially oncogenic pathways. 
In conclusion, the absence or knockdown of FLCN has widespread consequences (Figure 5). Most of these are not well understood and how they lead to the BHD phenotype remains to be determined. Therefore, we decided to return to the clinic to investigate if the BHD phenotype could provide us with clues about the pathophysiology.

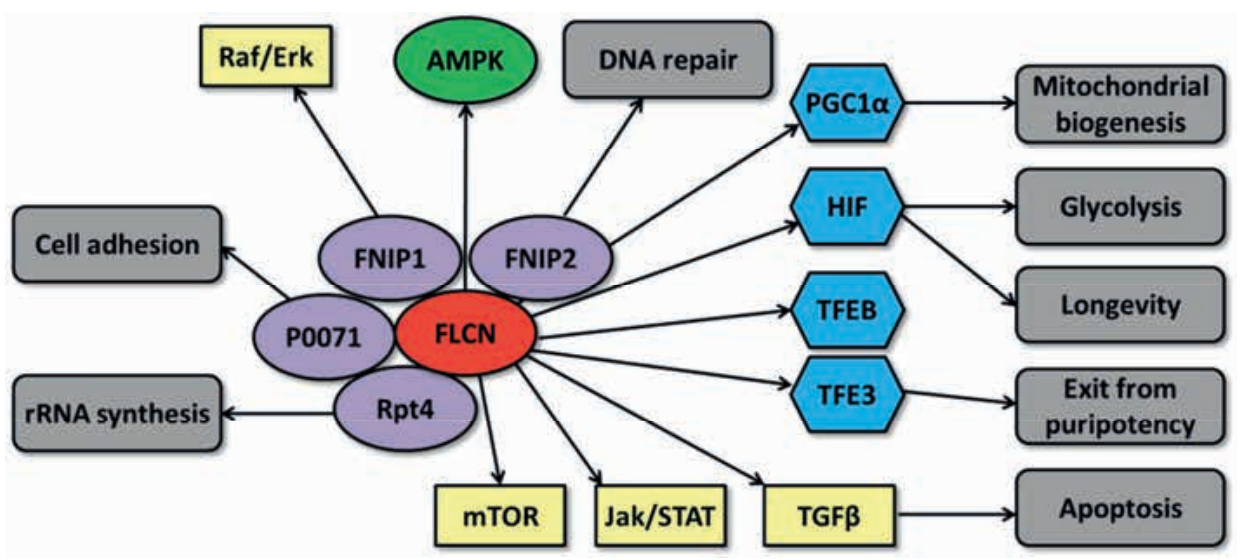

Figure 5 - FLCN-associated signalling pathways

Known interacting proteins (purple) are depicted around FLCN (red). Pathways and targets regulated by FLCN are indicated in yellow, blue and green. Biological processes known to be affected by FLCN are indicated in grey. Reviewed in $(72,73)$.

\section{The primary cilium}

In addition to the abovementioned characteristics of BHD we have observed that almost all patients develop cysts in organs beside the lung, including liver, pancreas and kidneys (unpublished data and (7)). This observation is supported by the mammalian BHD animal models, all of which develop cystic kidneys $(31,33$, 35-38). Development of cystic organs is one of the hallmarks of a large group of disorders caused by malfunctioning of the primary cilium, the so-called ciliopathies (74-76). This suggests a common pathogenic mechanism for these diseases and BHD.

The primary, immotile cilium is a conserved, solitary structure that projects from the apical cell surface of virtually all epithelial cells into the extracellular environment (Figure 6). It is composed of a central axoneme consisting of nine microtubule doublets in a "9+0 arrangement" in contrast to the motile cilia, which have a " $9+2$ arrangement" as the nine doublets surround a central microtubule pair (75). The axoneme is covered by a specialised plasma membrane which is continuous with the normal plasma membrane, but has a unique complement of proteins and lipids (77). The primary cilium is anchored to the cytoplasm by the basal body, which is a modified mother centriole (78). The primary cilium is a highly dynamic organelle that only assembles in quiescent or G1 cells. Cell cycle 
re-entry is preceded by cilium reabsorption before S phase or during G2 and after cells exit mitosis new cilia are formed. Ciliogenesis can only start after the establishment of apico-basal polarity by the formation of tight junctions along the basolateral membrane (79). Next, the mother centriole develops into the basal body by acquisition of (sub)distal appendages and docks to a ciliary vesicle, which fuses with the cell membrane (78). New cilia are then constructed from the basal body microtubules through an evolutionarily conserved process termed intraflagellar transport (IFT). Since the ciliary cytoplasm is devoid of ribosomes and separated from the cellular cytoplasm by the transition fibers, all proteins necessary for ciliary construction, maintenance and function are transported via bidirectional IFT along the axoneme $(75,80)$. Anterograde transport toward the ciliary tip is driven by kinesin- 2 and retrograde transport to the ciliary base is mediated by dynein coupled to different IFT protein complexes $(81,82)$.

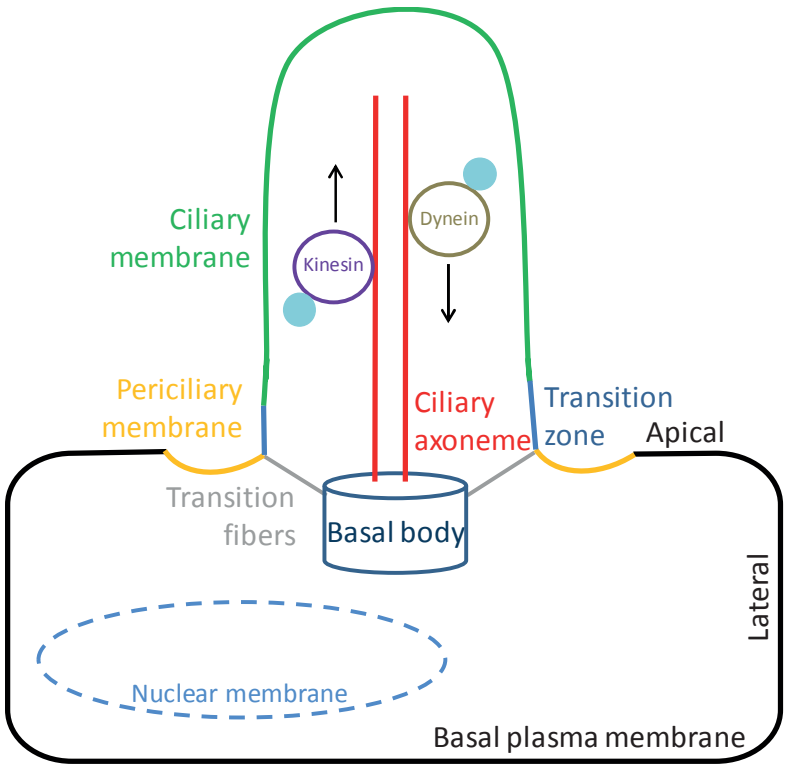

Figure 6 - Schematic of the primary cilium

Schematic of the primary cilium that projects from the apical cell surface and is covered by a specialised plasma membrane (green). The cilium is anchored to the plasma membrane (black) by the basal body (dark blue) via transition fibers (grey). Transport within the primary cilium takes place via intraflagellar transport along the axoneme (red) made up of microtubules driven by kinesin (purple) and dynein (grey) coupled to IFT complexes (light blue) for antero- and reterograde transport, respectively.

The function of the primary cilium is complex and versatile. The cilia are considered to be "cellular antennae" providing chemo- and mechanosensation. For example, in the kidney the primary cilia bend in response to fluid flow resulting in calcium influx followed by release of intracellular calcium stores from the endoplasmic reticulum (75). In addition, primary cilia provide transduction of various signalling pathways including the Hedgehog pathway, Hippo signalling, the canonical Wnt pathway and the non-canonical Wnt pathway under control of planar cell polarity (PCP) $(74,79,83)$. The latter is of special interest, as PCP regulates a form of polarity perpendicular to apico-basal polarity, which is also essential for ciliogenesis. In renal tubules, PCP dictates polarised cell division. The 
mitotic spindles in dividing renal cells are oriented along an axis that is parallel to the longitudinal axis of the tubule. In the normal kidney tubule, most of the cells divide within $34^{\circ}$ of the longitudinal axis of the tubule (84). This results in tubular elongation without changes in tubule diameter (85). Dysfunction of PCP results in disoriented cell division, leading to cyst development. During the past decade mutations in over 50 different genes have been identified that lead to development of ciliopathies $(80,86)$. In most cases ciliary function is disturbed due to functional loss or complete absence of a particular protein localising to the primary cilium, the basal body or both. However, proteins with different intracellular locations influencing ciliary function from a distance have also been shown to be causative of ciliopathies $(86,87)$.

It is of considerable interest in this regard that both tuberous sclerosis complex (TSC) and Von Hippel-Lindau disease (VHL) have recently been associated with malfunctioning of the primary cilium. TSC is caused by mutations in the TSC1 and TSC2 genes encoding hamartin and tuberin, respectively and VHL is caused by mutations in the VHL gene encoding the VHL protein (pVHL) $(62,88)$. Hamartin localises to the ciliary basal body, while tuberin interacts with proteins localised to the primary cilium and pVHL also localises to the primary cilium $(89,90)$. Also, combined loss of the mouse ortholog of pVHL with either Pten (91) or Gsk3 $\beta$ (92) resulted in loss of cilia and development of cysts in mouse tissues.

The clinical similarities between BHD and the ciliopathies, combined with the recent findings of TSC and VHL involvement in ciliary function and cell polarity, suggest a role for FLCN in these mechanisms. Therefore, we hypothesised that mutation of the FLCN gene in kidney cells leads to disruption of ciliary function, disturbing cell polarity and causing cyst development. This is investigated in Chapter 2.

\section{Zebrafish as an experimental animal model}

It has become clear that homozygous knockout of FLCN in vertebrates is embryonically lethal $(31,33-38)$, indicating that FLCN plays a vital role during embryogenesis. However, the exact role of FLCN during this process is difficult to determine in the currently established animal models. Therefore, we chose to generate a zebrafish model for Birt-Hogg-Dubé syndrome.

The zebrafish (Danio rerio) is a small tropical freshwater fish native to the shallow, slow-moving waters of the Indian subcontinent (93). During the past five decades zebrafish have been recognised by scientists as an excellent model to study gene function in both embryonic development and oncogenesis (94-96). They are robust, require minimal care and are small $(3-4 \mathrm{~cm})$, so approximately 40 adult animals can inhabit one 8 litre tank, resulting in low housing costs compared to other experimental animal models (94-96). Zebrafish are genetically very similar to humans; approximately $84 \%$ of human disease genes have a functioning 


\section{CHAPTER 1}

zebrafish ortholog or multiple homologs (97). The fish genome has undergone a whole genome duplication following the evolutionary divergence from mammals, resulting in the presence of multiple paralogs $(98,99)$. As they are vertebrates, zebrafish have nearly all mammal organs, including brain, eyes, heart, intestines, pancreas, kidney and liver (100). Zebrafish can develop a wide variety of both benign and malignant tumours in all these organs, with histology and gene expression similar to that of human tumours $(100,101)$. In addition, zebrafish are the only vertebrate in which it is possible to perform large-scale genetic screens, due to large offspring production and the easy of genetic manipulation.

One healthy zebrafish male-female pair can generate up to 300 eggs per breeding and can breed weekly. The female deposits the eggs, which are externally fertilised by the male. The fertilised eggs can be easily collected in breeding chambers (94). These eggs develop ex vivo at a rapid pace (Figure 7). At the optimal incubation temperature of $28.5^{\circ} \mathrm{C}$, zebrafish embryogenesis (extensively reviewed in (102)) is complete within the first three days after fertilisation, after which the zebrafish are referred to as "larvae". These developing structures can easily be visualised without dissection as the embryos are transparent up to one month of age (96). "Casper" mutant zebrafish lines are also available that remain transparent throughout life (103).

The ex vivo embryogenesis enables easy manipulation. Gene expression can be transiently inhibited in zebrafish embryos using morpholinos. These are antisense oligonucleotides of about 25 subunits with a structure similar to DNA, with the exception of a morpholino ring instead of a ribose ring (Figure 8). This causes them to be resistant to nucleases and as a result they are very stable. After injection into a single-cell stage embryo, morpholinos either block translation by targeting the 5' UTR through the first 25 bases of the coding sequence of the gene of interest or interfere with pre-mRNA splicing, by binding over the splice junction (104). Morpholinos remain active for about three days, well within the time period of organogenesis.

Zebrafish genetics has made it possible to express a gene of interest in a particular organ or tissue through use of a tissue-specific promoter (96). One example is the $\mathrm{Tg}(w t 1 b: E G F P)$ line which expresses enhanced green fluorescent protein (EGFP) exclusively in the kidney and the exocrine pancreas (105) and can therefore be used to monitor development of these organs in living embryos (Figure 9). Vertebrates develop three forms of kidneys, a pronephros, a mesonephros and a metanephros (106). During embryogenesis and early larval life the pronephros is the functional kidney, providing osmoregulation. The pronephros consists of two nephrons with glomeruli fused at the embryo midline just ventral to the dorsal aorta which lead into bilateral pronephric tubules and ducts. The latter end at the cloaca, which is located in the tail at the end of the yolk extension. 

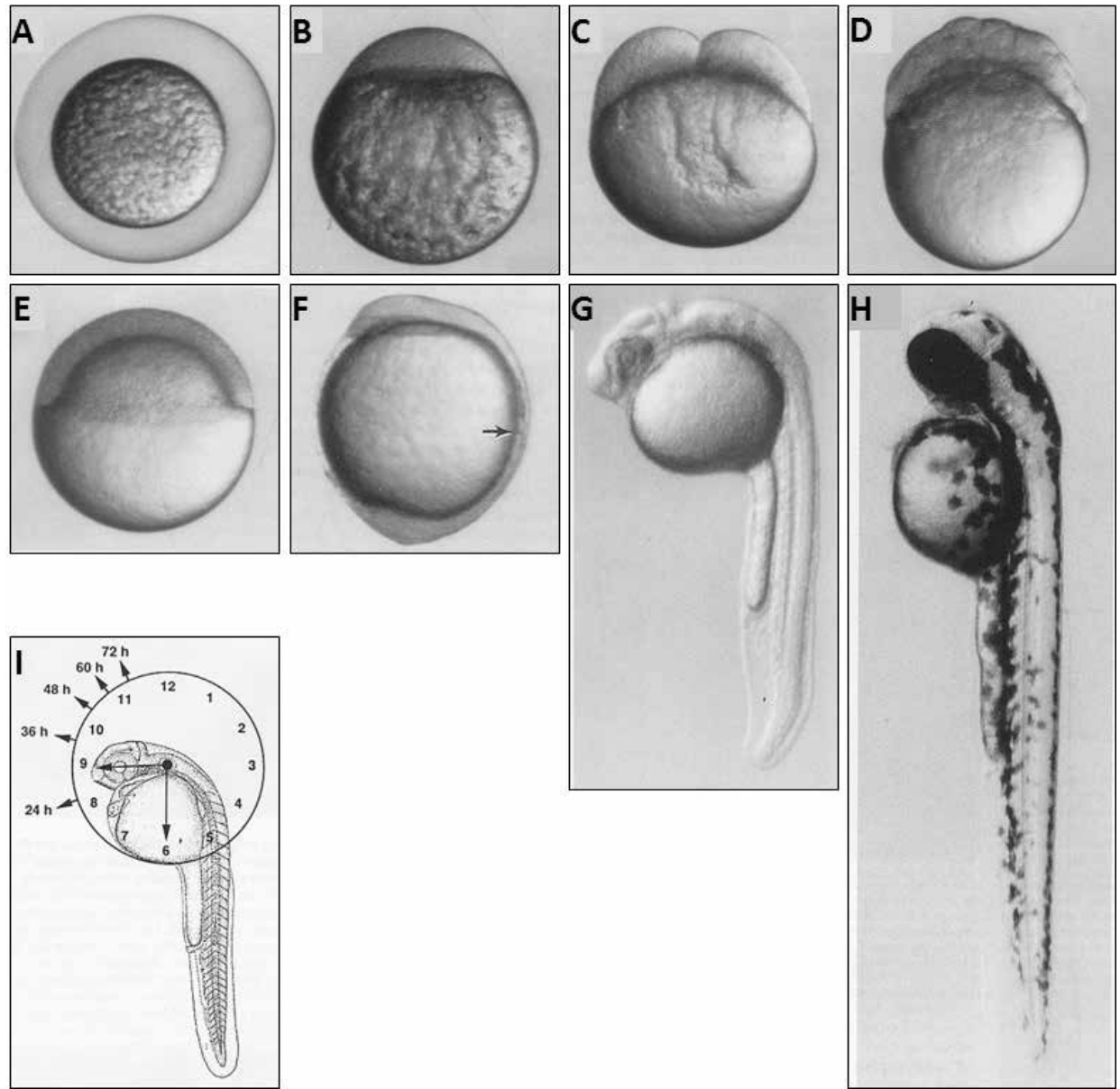

Figure 7 - Selected stage of zebrafish embryo development

(A) Zebrafish embryogenesis starts with the zygote period ( 0 - $3 / 4$ hours post fertilisation (hpf)) just after fertilisation. (B) This one-cell stage lasts until the first cleavage about 40 minutes after fertilisation. (C) Then the cleavage period ( $3 / 4-2 \frac{1}{4}$ hpf) starts, during which the cells divide every 15 minutes until 64cell stage (D). During the blastula period (21/4 - 51/4 hpf) which lasts from the 128-cell stage until the onset of gastrulation, zygotic gene transcription is activated and epiboly begins. (E) At $50 \%$ epiboly gastrulation begins (51/4 - $10 \mathrm{hpf}$ ) and the blastula, consisting of a single layer of cells is reorganised into the gastrula, consisting of the three germ layers ectoderm, mesoderm and endoderm. The embryonic axis is also determined. (F) During the segmentation period (10 - $24 \mathrm{hpf}$ ) the somites develop (indicated by arrow) and (G) the rudiments of the primary organs, such as the brain, pronephros and otic vesicle become visible. (H) After somitogenesis the pharyngula period (24 - 48 hpf) starts which is characterised by development of the pigment cells, retina, fins, circulatory system, the pharyngeal arches (that will become the jaws and gills) and the primordia of the liver and swim bladder. (I) In addition, the heart beat begins and the head straightens out. At $48 \mathrm{hpf}$ the hatching period begins (48 $72 \mathrm{hpf}$ ) when the yolk shrinks and morphogenesis of organ rudiments is complete, apart from the gut and related structures. Adapted from (102) with permission from Wiley. 
A

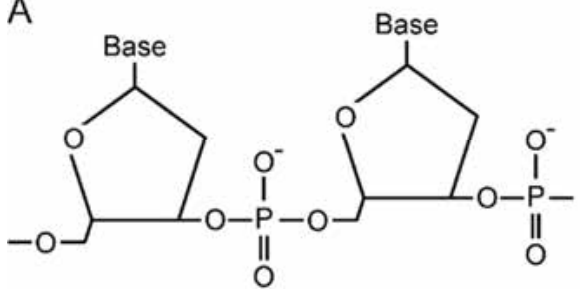

B

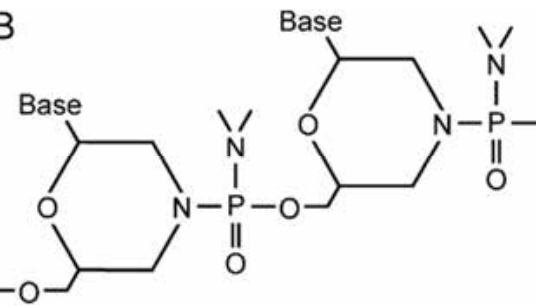

Figure 8 - Structure of morpholino oligonucleotides

(A) DNA structure (B) morpholino structure. Republished from (104) with permission of the Company of Biologists conveyed through Copyright Clearance Center, Inc.

Zebrafish are very well suited for the study of cilia and the phenotypes associated with their disruption. Several embryonic zebrafish structures show formation of cilia, including the Kupffer's vesicle, the pronephric duct and the otic vesicle (107109), which can be imaged by immunofluorescence confocal microscopy using acetylated alpha tubulin antibodies on whole embryos. Zebrafish with knockdown of known ciliary genes, caused by either mutation (mutants) or morpholino injection (morphants), share a common phenotype characterised by curved body axis, hydrocephalus and cyst formation in the pronephros (110-112).

In conclusion, the zebrafish is a valuable vertebrate system in which to elucidate molecular mechanisms of development and carcinogenesis as both zebrafish genetics and tumour formation is very similar to humans. Rapid development of large numbers of transparent zebrafish embryos ex vivo enables easy visualisation and manipulation of organogenesis. In addition, zebrafish are very well suited for the study of cilia and the phenotypes associated with their disruption. This is why we decided to generate a zebrafish model for BHD as characterised in Chapter 3.
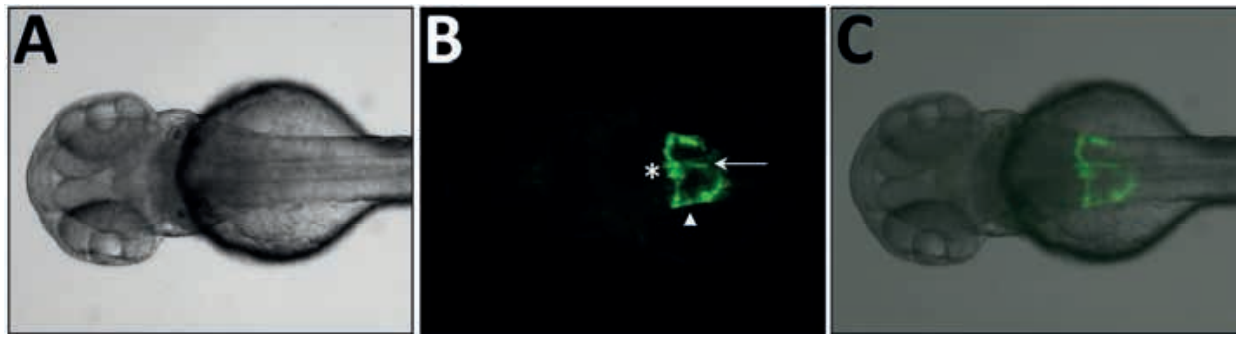

Figure 9 - Example of a transgenic zebrafish line

48 hours post fertilisation $\mathrm{Tg}(w t 1 b: E G F P)$ zebrafish embryo. (A) Brightfield. (B) EGFP expression is restricted to the glomeruli (asterisk), pronephric tubuli (arrowhead) and exocrine pancreas (arrow). (C) Merge. 


\section{FLCN-associated skin lesions}

The skin phenotype of BHD has been the subject of some controversy. In the original report by Birt, Hogg and Dubé the skin lesions were described as fibrofolliculomas associated with trichodiscomas and acrochordons (2). Acrochordons (also known as skin tags) are harmless benign tumours that do not contain any hair follicles or sweat ducts (113). As skin tags are frequent in the general population (114), it is unclear if they are an actual symptom of BHD. Fibrofolliculomas develop in approximately $80 \%$ of BHD patients and were first described as branching strands of epithelial cells centred around an often distorted hair follicle, that is sometimes dilated to form a keratin-filled cyst (2). Therefore, the general consensus is that they are benign hair follicle tumours. However, we rather think that they are derived from the sebaceous gland, as we consistently see that the typical fibrofolliculoma epithelial strands are continuous with the sebaceous glands $(115,116)$. This could also explain why the lesions are usually localised to areas with a high concentration of sebaceous glands, such as the perinasal skin $(115,116)$.

The trichodiscomas were described as dome-shaped, sharply circumscribed lesions containing well-vascularised fibrous tissue with a hair follicle in the periphery of the lesion (2). They are thought to be derived from the hair disk (2, 113), although there is little evidence for this, causing some authors to argue that the fibrofolliculomas and trichodiscomas in BHD are the same lesion (reviewed in (117)). They explain the difference in histology by variations in sectioning and clinical bias in tissue sampling. However, in 1985 Starink et al. reported on a family with "trichodiscomas" already presenting at childhood, in contrast to BHD lesions, which typically do not develop until the third decade of life $(2,118)$. They recently determined that these lesions present as fibrovascular tumours with a hair follicle located at the margin of the lesion at all sectioning levels (119). In addition, development of these lesions is not linked to the FLCN locus and therefore they suggest to rename them familial multiple discoid fibromas (FMDF; OMIM $\% 190340)$, to avoid any further confusion with the fibrofolliculomas/trichodiscomas in BHD (119).

As these discoid fibromas are clinically indistinguishable from the skin lesions in BHD, we hypothesised them to be caused by a mutation in one of the FLCN interacting proteins, FNIP1 or FNIP2. Our work on this hypothesis is further described in Chapter 4. 


\section{Aims of the thesis}

The aim of the present thesis is to gain more insight into the molecular functioning of FLCN in order to better understand BHD pathophysiology.

As the BHD phenotype is clinically similar to the ciliopathies, we investigate the role of FLCN in the primary cilium in Chapter 2.

Studies using experimental animal models have shown that homozygous knockout of FLCN is embryonically lethal. However, investigation of developmental defects is challenging in the currently established murine and canine models. Chapter 3 describes development of a novel animal model for BHD in zebrafish, to study the role of FLCN in embryogenesis.

The cutaneous BHD phenotype bears resemblance to FMDF, suggesting a role for FLCN interacting proteins FNIP1 or FNIP2 in FMDF pathophysiology. This was also investigated using a zebrafish knockdown model described in Chapter 4. 


\section{References}

1. Hornstein OP, Knickenberg M. Perifollicular fibromatosis cutis with polyps of the colon--a cutaneo-intestinal syndrome sui generis. Archives for dermatological research Archiv fur dermatologische Forschung. 1975 Sep 12;253(2):161-75. PubMed PMID: 1200700.

2. Birt AR, Hogg GR, Dube WJ. Hereditary multiple fibrofolliculomas with trichodiscomas and acrochordons. Archives of dermatology. 1977 Dec;113(12):1674-7. PubMed PMID: 596896.

3. Schulz T, Hartschuh W. Birt-Hogg-Dube syndrome and Hornstein-Knickenberg syndrome are the same. Different sectioning technique as the cause of different histology. Journal of cutaneous pathology. 1999 Jan;26(1):55-61. PubMed PMID: 10189247.

4. Happle R. Hornstein-Birt-Hogg-Dube syndrome: a renaming and reconsideration. American journal of medical genetics Part A. 2012 Jun;158A(6):1247-51. PubMed PMID: 22581760.

5. Schmidt LS, Nickerson ML, Warren MB, Glenn GM, Toro JR, Merino MJ, et al. Germline BHD-mutation spectrum and phenotype analysis of a large cohort of families with BirtHogg-Dube syndrome. American journal of human genetics. 2005 Jun;76(6):1023-33. PubMed PMID: 15852235. Pubmed Central PMCID: 1196440.

6. Toro JR, Wei MH, Glenn GM, Weinreich M, Toure O, Vocke C, et al. BHD mutations, clinical and molecular genetic investigations of Birt-Hogg-Dube syndrome: a new series of 50 families and a review of published reports. Journal of medical genetics. 2008 Jun;45(6):321-31. PubMed PMID: 18234728. Pubmed Central PMCID: 2564862.

7. Kluger N, Giraud S, Coupier I, Avril MF, Dereure O, Guillot B, et al. Birt-Hogg-Dube syndrome: clinical and genetic studies of 10 French families. The British journal of dermatology. 2010 Mar;162(3):527-37. PubMed PMID: 19785621.

8. Houweling AC, Gijezen LM, Jonker MA, van Doorn MB, Oldenburg RA, van SpaendonckZwarts KY, et al. Renal cancer and pneumothorax risk in Birt-Hogg-Dube syndrome; an analysis of 115 FLCN mutation carriers from 35 BHD families. British journal of cancer. 2011 Dec 6;105(12):1912-9. PubMed PMID: 22146830. Pubmed Central PMCID: 3251884.

9. Khoo SK, Giraud S, Kahnoski K, Chen J, Motorna O, Nickolov R, et al. Clinical and genetic studies of Birt-Hogg-Dube syndrome. Journal of medical genetics. 2002 Dec;39(12):906-12. PubMed PMID: 12471204. Pubmed Central PMCID: 1757219.

10. Menko FH, van Steensel MA, Giraud S, Friis-Hansen L, Richard S, Ungari S, et al. BirtHogg-Dube syndrome: diagnosis and management. The lancet oncology. 2009 Dec;10(12):1199-206. PubMed PMID: 19959076.

11. Zbar B, Alvord WG, Glenn G, Turner M, Pavlovich CP, Schmidt L, et al. Risk of renal and colonic neoplasms and spontaneous pneumothorax in the Birt-Hogg-Dube syndrome. Cancer epidemiology, biomarkers \& prevention : a publication of the American Association for Cancer Research, cosponsored by the American Society of Preventive Oncology. 2002 Apr;11(4):393-400. PubMed PMID: 11927500.

12. Pavlovich CP, Walther MM, Eyler RA, Hewitt SM, Zbar B, Linehan WM, et al. Renal tumors in the Birt-Hogg-Dube syndrome. The American journal of surgical pathology. 2002 Dec;26(12):1542-52. PubMed PMID: 12459621.

13. Leter EM, Koopmans AK, Gille JJ, van Os TA, Vittoz GG, David EF, et al. Birt-Hogg-Dube syndrome: clinical and genetic studies of 20 families. The Journal of investigative dermatology. 2008 Jan;128(1):45-9. PubMed PMID: 17611575.

14. Pavlovich CP, Grubb RL, 3rd, Hurley K, Glenn GM, Toro J, Schmidt LS, et al. Evaluation and management of renal tumors in the Birt-Hogg-Dube syndrome. The Journal of urology. 2005 May;173(5):1482-6. PubMed PMID: 15821464. 
15. Nahorski MS, Lim DH, Martin L, Gille JJ, McKay K, Rehal PK, et al. Investigation of the Birt-Hogg-Dube tumour suppressor gene (FLCN) in familial and sporadic colorectal cancer. Journal of medical genetics. 2010 Jun;47(6):385-90. PubMed PMID: 20522427.

16. Chung JY, Ramos-Caro FA, Beers B, Ford MJ, Flowers F. Multiple lipomas, angiolipomas, and parathyroid adenomas in a patient with Birt-Hogg-Dube syndrome. International journal of dermatology. 1996 May;35(5):365-7. PubMed PMID: 8734663.

17. Liu V, Kwan T, Page EH. Parotid oncocytoma in the Birt-Hogg-Dube syndrome. Journal of the American Academy of Dermatology. 2000 Dec;43(6):1120-2. PubMed PMID: 11100034.

18. Linehan WM, Walther MM, Zbar B. The genetic basis of cancer of the kidney. The Journal of urology. 2003 Dec;170(6 Pt 1):2163-72. PubMed PMID: 14634372.

19. Schmidt LS, Warren MB, Nickerson ML, Weirich G, Matrosova V, Toro JR, et al. BirtHogg-Dube syndrome, a genodermatosis associated with spontaneous pneumothorax and kidney neoplasia, maps to chromosome 17p11.2. American journal of human genetics. 2001 Oct;69(4):876-82. PubMed PMID: 11533913. Pubmed Central PMCID: 1226073.

20. Khoo SK, Bradley M, Wong FK, Hedblad MA, Nordenskjold M, Teh BT. Birt-Hogg-Dube syndrome: mapping of a novel hereditary neoplasia gene to chromosome $17 \mathrm{p} 12$ q11.2. Oncogene. 2001 Aug 23;20(37):5239-42. PubMed PMID: 11526515.

21. Nickerson ML, Warren MB, Toro JR, Matrosova V, Glenn G, Turner ML, et al. Mutations in a novel gene lead to kidney tumors, lung wall defects, and benign tumors of the hair follicle in patients with the Birt-Hogg-Dube syndrome. Cancer cell. 2002 Aug;2(2):15764. PubMed PMID: 12204536.

22. Wei MH, Blake PW, Shevchenko J, Toro JR. The folliculin mutation database: an online database of mutations associated with Birt-Hogg-Dube syndrome. Human mutation. 2009 Sep;30(9):E880-90. PubMed PMID: 19562744. Pubmed Central PMCID: 3234166.

23. Lim DH, Rehal PK, Nahorski MS, Macdonald F, Claessens T, Van Geel M, et al. A new locus-specific database (LSDB) for mutations in the folliculin (FLCN) gene. Human mutation. 2010 Jan;31(1):E1043-51. PubMed PMID: 19802896.

24. Farrant PB, Emerson R. Letter: hyfrecation and curettage as a treatment for fibrofolliculomas in Birt-Hogg-Dube syndrome. Dermatologic surgery : official publication for American Society for Dermatologic Surgery [et al]. 2007 Oct;33(10):1287-8. PubMed PMID: 17903168.

25. Jacob CI, Dover JS. Birt-Hogg-Dube syndrome: treatment of cutaneous manifestations with laser skin resurfacing. Archives of dermatology. 2001 Jan;137(1):98-9. PubMed PMID: 11176677.

26. Toro JR, Pautler SE, Stewart L, Glenn GM, Weinreich M, Toure O, et al. Lung cysts, spontaneous pneumothorax, and genetic associations in 89 families with Birt-HoggDube syndrome. American journal of respiratory and critical care medicine. 2007 May 15;175(10):1044-53. PubMed PMID: 17322109. Pubmed Central PMCID: 1899269.

27. Liu W, Chen Z, Ma Y, Wu X, Jin Y, Hou S. Genetic characterization of the Drosophila birthogg-dube syndrome gene. PloS one. 2013;8(6):e65869. PubMed PMID: 23799055. Pubmed Central PMCID: 3684598.

28. Warren MB, Torres-Cabala CA, Turner ML, Merino MJ, Matrosova VY, Nickerson ML, et al. Expression of Birt-Hogg-Dube gene mRNA in normal and neoplastic human tissues. Modern pathology : an official journal of the United States and Canadian Academy of Pathology, Inc. 2004 Aug;17(8):998-1011. PubMed PMID: 15143337.

29. Vocke CD, Yang Y, Pavlovich CP, Schmidt LS, Nickerson ML, Torres-Cabala CA, et al. High frequency of somatic frameshift BHD gene mutations in Birt-Hogg-Dubeassociated renal tumors. Journal of the National Cancer Institute. 2005 Jun 15;97(12):931-5. PubMed PMID: 15956655. 
30. Kouchi M, Okimoto K, Matsumoto I, Tanaka K, Yasuba M, Hino O. Natural history of the Nihon (Bhd gene mutant) rat, a novel model for human Birt-Hogg-Dube syndrome. Virchows Archiv : an international journal of pathology. 2006 Apr;448(4):463-71. PubMed PMID: 16447066.

31. Okimoto K, Sakurai J, Kobayashi T, Mitani H, Hirayama Y, Nickerson ML, et al. A germline insertion in the Birt-Hogg-Dube (BHD) gene gives rise to the Nihon rat model of inherited renal cancer. Proceedings of the National Academy of Sciences of the United States of America. 2004 Feb 17;101(7):2023-7. PubMed PMID: 14769940. Pubmed Central PMCID: 357045.

32. Lium B, Moe L. Hereditary multifocal renal cystadenocarcinomas and nodular dermatofibrosis in the German shepherd dog: macroscopic and histopathologic changes. Veterinary pathology. 1985 Sep;22(5):447-55. PubMed PMID: 4049673.

33. Lingaas F, Comstock KE, Kirkness EF, Sorensen A, Aarskaug T, Hitte C, et al. A mutation in the canine BHD gene is associated with hereditary multifocal renal cystadenocarcinoma and nodular dermatofibrosis in the German Shepherd dog. Human molecular genetics. 2003 Dec 1;12(23):3043-53. PubMed PMID: 14532326.

34. Cash TP, Gruber JJ, Hartman TR, Henske EP, Simon MC. Loss of the Birt-Hogg-Dube tumor suppressor results in apoptotic resistance due to aberrant TGFbeta-mediated transcription. Oncogene. 2011 Jun 2;30(22):2534-46. PubMed PMID: 21258407. Pubmed Central PMCID: 3109270.

35. Hartman TR, Nicolas E, Klein-Szanto A, Al-Saleem T, Cash TP, Simon MC, et al. The role of the Birt-Hogg-Dube protein in mTOR activation and renal tumorigenesis. Oncogene. 2009 Apr 2;28(13):1594-604. PubMed PMID: 19234517. Pubmed Central PMCID: 2664853.

36. Hasumi Y, Baba M, Ajima R, Hasumi H, Valera VA, Klein ME, et al. Homozygous loss of BHD causes early embryonic lethality and kidney tumor development with activation of mTORC1 and mTORC2. Proceedings of the National Academy of Sciences of the United States of America. 2009 Nov 3;106(44):18722-7. PubMed PMID: 19850877. Pubmed Central PMCID: 2765925.

37. Baba M, Furihata M, Hong SB, Tessarollo L, Haines DC, Southon E, et al. Kidneytargeted Birt-Hogg-Dube gene inactivation in a mouse model: Erk1/2 and Akt-mTOR activation, cell hyperproliferation, and polycystic kidneys. Journal of the National Cancer Institute. 2008 Jan 16;100(2):140-54. PubMed PMID: 18182616. Pubmed Central PMCID: 2704336.

38. Chen J, Futami K, Petillo D, Peng J, Wang P, Knol J, et al. Deficiency of FLCN in mouse kidney led to development of polycystic kidneys and renal neoplasia. PloS one. 2008;3(10):e3581. PubMed PMID: 18974783. Pubmed Central PMCID: 2570491.

39. Bonsdorff TB, Jansen JH, Lingaas F. Second hits in the FLCN gene in a hereditary renal cancer syndrome in dogs. Mammalian genome : official journal of the International Mammalian Genome Society. 2008 Feb;19(2):121-6. PubMed PMID: 18219524.

40. Knudson AG, Jr. Mutation and cancer: statistical study of retinoblastoma. Proceedings of the National Academy of Sciences of the United States of America. 1971 Apr;68(4):820-3. PubMed PMID: 5279523. Pubmed Central PMCID: 389051.

41. Yang Y, Padilla-Nash HM, Vira MA, Abu-Asab MS, Val D, Worrell R, et al. The UOK 257 cell line: a novel model for studies of the human Birt-Hogg-Dube gene pathway. Cancer genetics and cytogenetics. 2008 Jan 15;180(2):100-9. PubMed PMID: 18206534. Pubmed Central PMCID: 2440670.

42. Hong SB, Oh H, Valera VA, Stull J, Ngo DT, Baba M, et al. Tumor suppressor FLCN inhibits tumorigenesis of a FLCN-null renal cancer cell line and regulates expression of key molecules in TGF-beta signaling. Molecular cancer. 2010;9:160. PubMed PMID: 20573232. Pubmed Central PMCID: 2907329. 
43. Sievers F, Wilm A, Dineen D, Gibson TJ, Karplus K, Li W, et al. Fast, scalable generation of high-quality protein multiple sequence alignments using Clustal Omega. Molecular systems biology. 2011;7:539. PubMed PMID: 21988835. Pubmed Central PMCID: 3261699.

44. Goujon M, McWilliam H, Li W, Valentin F, Squizzato S, Paern J, et al. A new bioinformatics analysis tools framework at EMBL-EBI. Nucleic acids research. 2010 Jul;38(Web Server issue):W695-9. PubMed PMID: 20439314. Pubmed Central PMCID: 2896090.

45. Waterhouse AM, Procter JB, Martin DM, Clamp M, Barton GJ. Jalview Version 2--a multiple sequence alignment editor and analysis workbench. Bioinformatics. 2009 May 1;25(9):1189-91. PubMed PMID: 19151095. Pubmed Central PMCID: 2672624.

46. Krymskaya VP, Goncharova EA. PI3K/mTORC1 activation in hamartoma syndromes: therapeutic prospects. Cell cycle. 2009 Feb 1;8(3):403-13. PubMed PMID: 19177005. Pubmed Central PMCID: 3718392.

47. Baba M, Hong SB, Sharma N, Warren MB, Nickerson ML, Iwamatsu A, et al. Folliculin encoded by the BHD gene interacts with a binding protein, FNIP1, and AMPK, and is involved in AMPK and mTOR signaling. Proceedings of the National Academy of Sciences of the United States of America. 2006 Oct 17;103(42):15552-7. PubMed PMID: 17028174. Pubmed Central PMCID: 1592464.

48. Hudon V, Sabourin S, Dydensborg AB, Kottis V, Ghazi A, Paquet M, et al. Renal tumour suppressor function of the Birt-Hogg-Dube syndrome gene product folliculin. Journal of medical genetics. 2010 Mar;47(3):182-9. PubMed PMID: 19843504.

49. van Slegtenhorst M, Khabibullin D, Hartman TR, Nicolas E, Kruger WD, Henske EP. The Birt-Hogg-Dube and tuberous sclerosis complex homologs have opposing roles in amino acid homeostasis in Schizosaccharomyces pombe. The Journal of biological chemistry. 2007 Aug 24;282(34):24583-90. PubMed PMID: 17556368.

50. Claessens T, Weppler SA, van Geel M, Creytens D, Vreeburg M, Wouters B, et al. Neuroendocrine carcinoma in a patient with Birt-Hogg-Dube syndrome. Nature reviews Urology. 2010 Oct;7(10):583-7. PubMed PMID: 20842188.

51. Gijezen LM, Vernooij M, Martens H, Oduber CE, Henquet CJ, Starink TM, et al. Topical Rapamycin as a Treatment for Fibrofolliculomas in Birt-Hogg-Dube Syndrome: A Double-Blind Placebo-Controlled Randomized Split-Face Trial. PloS one. 2014;9(6):e99071. PubMed PMID: 24910976.

52. Shaw RJ. LKB1 and AMP-activated protein kinase control of mTOR signalling and growth. Acta physiologica. 2009 May;196(1):65-80. PubMed PMID: 19245654. Pubmed Central PMCID: 2760308.

53. Hasumi H, Baba M, Hong SB, Hasumi Y, Huang Y, Yao M, et al. Identification and characterization of a novel folliculin-interacting protein FNIP2. Gene. 2008 May 31;415(1-2):60-7. PubMed PMID: 18403135. Pubmed Central PMCID: 2727720.

54. Takagi Y, Kobayashi T, Shiono M, Wang L, Piao X, Sun G, et al. Interaction of folliculin (Birt-Hogg-Dube gene product) with a novel Fnip1-like (FnipL/Fnip2) protein. Oncogene. 2008 Sep 11;27(40):5339-47. PubMed PMID: 18663353.

55. Sano S, Sakagami R, Sekiguchi M, Hidaka M. Stabilization of MAPO1 by specific binding with folliculin and AMP-activated protein kinase in $O(6)$-methylguanine-induced apoptosis. Biochemical and biophysical research communications. 2013 Jan 11;430(2):810-5. PubMed PMID: 23201403.

56. Nahorski MS, Seabra L, Straatman-Iwanowska A, Wingenfeld A, Reiman A, Lu X, et al. Folliculin interacts with p0071 (plakophilin-4) and deficiency is associated with disordered RhoA signalling, epithelial polarization and cytokinesis. Human molecular genetics. 2012 Dec 15;21(24):5268-79. PubMed PMID: 22965878. Pubmed Central PMCID: 3755511. 
57. Medvetz DA, Khabibullin D, Hariharan V, Ongusaha PP, Goncharova EA, Schlechter T, et al. Folliculin, the product of the Birt-Hogg-Dube tumor suppressor gene, interacts with the adherens junction protein p0071 to regulate cell-cell adhesion. PloS one. 2012;7(11):e47842. PubMed PMID: 23139756. Pubmed Central PMCID: 3490959.

58. Gaur K, Li J, Wang D, Dutta P, Yan SJ, Tsurumi A, et al. The Birt-Hogg-Dube tumor suppressor Folliculin negatively regulates ribosomal RNA synthesis. Human molecular genetics. 2013 Jan 15;22(2):284-99. PubMed PMID: 23077212. Pubmed Central PMCID: 3526160.

59. Singh SR, Zhen W, Zheng Z, Wang H, Oh SW, Liu W, et al. The Drosophila homolog of the human tumor suppressor gene BHD interacts with the JAK-STAT and Dpp signaling pathways in regulating male germline stem cell maintenance. Oncogene. 2006 Sep 28;25(44):5933-41. PubMed PMID: 16636660.

60. Gharbi H, Fabretti F, Bharill P, Rinschen MM, Brinkkotter S, Frommolt P, et al. Loss of the Birt-Hogg-Dube gene product folliculin induces longevity in a hypoxia-inducible factor-dependent manner. Aging cell. 2013 Aug;12(4):593-603. PubMed PMID: 23566034.

61. Preston RS, Philp A, Claessens T, Gijezen L, Dydensborg AB, Dunlop EA, et al. Absence of the Birt-Hogg-Dube gene product is associated with increased hypoxia-inducible factor transcriptional activity and a loss of metabolic flexibility. Oncogene. $2011 \mathrm{Mar}$ 10;30(10):1159-73. PubMed PMID: 21057536. Pubmed Central PMCID: 3787473.

62. Kaelin WG, Jr. The von Hippel-Lindau tumour suppressor protein: 02 sensing and cancer. Nature reviews Cancer. 2008 Nov;8(11):865-73. PubMed PMID: 18923434.

63. Zhang H, Gao P, Fukuda R, Kumar G, Krishnamachary B, Zeller KI, et al. HIF-1 inhibits mitochondrial biogenesis and cellular respiration in VHL-deficient renal cell carcinoma by repression of C-MYC activity. Cancer cell. 2007 May;11(5):407-20. PubMed PMID: 17482131.

64. Klomp JA, Petillo D, Niemi NM, Dykema KJ, Chen J, Yang XJ, et al. Birt-Hogg-Dube renal tumors are genetically distinct from other renal neoplasias and are associated with upregulation of mitochondrial gene expression. BMC medical genomics. 2010;3:59. PubMed PMID: 21162720. Pubmed Central PMCID: 3012009.

65. Krishnan B, Truong LD. Renal epithelial neoplasms: the diagnostic implications of electron microscopic study in 55 cases. Hum Pathol. 2002 Jan;33(1):68-79. PubMed PMID: 11823975. Epub 2002/02/02. eng.

66. Hong SB, Oh H, Valera VA, Stull J, Ngo DT, Baba M, et al. Tumor suppressor FLCN inhibits tumorigenesis of a FLCN-null renal cancer cell line and regulates expression of key molecules in TGF-beta signaling. Mol Cancer.9:160. PubMed PMID: 20573232. Pubmed Central PMCID: 2907329. Epub 2010/06/25. eng.

67. Cash TP, Gruber JJ, Hartman TR, Henske EP, Simon MC. Loss of the Birt-Hogg-Dube tumor suppressor results in apoptotic resistance due to aberrant TGFbeta-mediated transcription. Oncogene. 2011 Jan 24. PubMed PMID: 21258407. Epub 2011/01/25. Eng.

68. Grinberg AV, Kerppola T. Both Max and TFE3 cooperate with Smad proteins to bind the plasminogen activator inhibitor-1 promoter, but they have opposite effects on transcriptional activity. J Biol Chem. 2003 Mar 28;278(13):11227-36. PubMed PMID: 12551947. Epub 2003/01/29. eng.

69. Hong SB, Oh H, Valera VA, Baba M, Schmidt LS, Linehan WM. Inactivation of the FLCN tumor suppressor gene induces TFE3 transcriptional activity by increasing its nuclear localization. PloS one. 2010;5(12):e15793. PubMed PMID: 21209915. Pubmed Central PMCID: 3012117. 


\section{CHAPTER 1}

70. Betschinger J, Nichols J, Dietmann S, Corrin PD, Paddison PJ, Smith A. Exit from pluripotency is gated by intracellular redistribution of the bHLH transcription factor Tfe3. Cell. 2013 Apr 11;153(2):335-47. PubMed PMID: 23582324. Pubmed Central PMCID: 3661979.

71. Petit CS, Roczniak-Ferguson A, Ferguson SM. Recruitment of folliculin to lysosomes supports the amino acid-dependent activation of Rag GTPases. The Journal of cell biology. 2013 Sep 30;202(7):1107-22. PubMed PMID: 24081491. Pubmed Central PMCID: 3787382.

72. Tee AR, Pause A. Birt-Hogg-Dube: tumour suppressor function and signalling dynamics central to folliculin. Familial cancer. 2013 Sep;12(3):367-72. PubMed PMID: 23096221.

73. Schmidt LS. Birt-Hogg-Dube syndrome: from gene discovery to molecularly targeted therapies. Familial cancer. 2013 Sep;12(3):357-64. PubMed PMID: 23108783. Pubmed Central PMCID: 3637987.

74. Tobin JL, Beales PL. The nonmotile ciliopathies. Genet Med. 2009 Jun;11(6):386-402. PubMed PMID: 19421068.

75. Davenport JR, Yoder BK. An incredible decade for the primary cilium: a look at a onceforgotten organelle. American journal of physiology Renal physiology. 2005 Dec;289(6):F1159-69. PubMed PMID: 16275743.

76. Arts HH, Knoers NV. Current insights into renal ciliopathies: what can genetics teach us? Pediatric nephrology. 2013 Jun;28(6):863-74. PubMed PMID: 22829176. Pubmed Central PMCID: 3631122.

77. Pazour GJ. Intraflagellar transport and cilia-dependent renal disease: the ciliary hypothesis of polycystic kidney disease. Journal of the American Society of Nephrology : JASN. 2004 Oct;15(10):2528-36. PubMed PMID: 15466257.

78. Sorokin S. Centrioles and the formation of rudimentary cilia by fibroblasts and smooth muscle cells. The Journal of cell biology. 1962 Nov;15:363-77. PubMed PMID: 13978319. Pubmed Central PMCID: 2106144.

79. Adams M, Smith UM, Logan CV, Johnson CA. Recent advances in the molecular pathology, cell biology and genetics of ciliopathies. Journal of medical genetics. 2008 May;45(5):257-67. PubMed PMID: 18178628.

80. Basten SG, Giles RH. Functional aspects of primary cilia in signaling, cell cycle and tumorigenesis. Cilia. 2013;2(1):6. PubMed PMID: 23628112. Pubmed Central PMCID: 3662159.

81. Garcia-Gonzalo FR, Reiter JF. Scoring a backstage pass: mechanisms of ciliogenesis and ciliary access. The Journal of cell biology. 2012 Jun 11;197(6):697-709. PubMed PMID: 22689651. Pubmed Central PMCID: 3373398.

82. Avasthi P, Marshall WF. Stages of ciliogenesis and regulation of ciliary length. Differentiation; research in biological diversity. 2012 Feb;83(2):S30-42. PubMed PMID: 22178116. Pubmed Central PMCID: 3269565.

83. D'Angelo A, Franco B. The dynamic cilium in human diseases. Pathogenetics. 2009;2(1):3. PubMed PMID: 19439065.

84. Fischer E, Legue E, Doyen A, Nato F, Nicolas JF, Torres V, et al. Defective planar cell polarity in polycystic kidney disease. Nat Genet. 2006 Jan;38(1):21-3. PubMed PMID: 16341222.

85. McNeill H. Planar cell polarity and the kidney. Journal of the American Society of Nephrology : JASN. 2009 Oct;20(10):2104-11. PubMed PMID: 19762494.

86. Hurd TW, Hildebrandt F. Mechanisms of nephronophthisis and related ciliopathies. Nephron Experimental nephrology. 2011;118(1):e9-14. PubMed PMID: 21071979. Pubmed Central PMCID: 2992643. 
87. Sang L, Miller JJ, Corbit KC, Giles RH, Brauer MJ, Otto EA, et al. Mapping the NPHP-JBTSMKS protein network reveals ciliopathy disease genes and pathways. Cell. 2011 May 13;145(4):513-28. PubMed PMID: 21565611. Pubmed Central PMCID: 3383065.

88. Curatolo P, Bombardieri R, Jozwiak S. Tuberous sclerosis. Lancet. 2008 Aug 23;372(9639):657-68. PubMed PMID: 18722871.

89. Bonnet CS, Aldred M, von Ruhland C, Harris R, Sandford R, Cheadle JP. Defects in cell polarity underlie TSC and ADPKD-associated cystogenesis. Hum Mol Genet. 2009 Jun 15;18(12):2166-76. PubMed PMID: 19321600.

90. Schermer B, Ghenoiu C, Bartram M, Muller RU, Kotsis F, Hohne M, et al. The von Hippel-Lindau tumor suppressor protein controls ciliogenesis by orienting microtubule growth. The Journal of cell biology. 2006 Nov 20;175(4):547-54. PubMed PMID: 17101696. Pubmed Central PMCID: 2064591.

91. Frew IJ, Thoma CR, Georgiev S, Minola A, Hitz M, Montani M, et al. pVHL and PTEN tumour suppressor proteins cooperatively suppress kidney cyst formation. The EMBO journal. 2008 Jun 18;27(12):1747-57. PubMed PMID: 18497742. Pubmed Central PMCID: 2435131.

92. Thoma CR, Frew IJ, Hoerner CR, Montani M, Moch H, Krek W. pVHL and GSK3beta are components of a primary cilium-maintenance signalling network. Nature cell biology. 2007 May;9(5):588-95. PubMed PMID: 17450132.

93. Arunachalam M, Raja M, Vijayakumar C, Malaiammal P, Mayden RL. Natural history of zebrafish (Danio rerio) in India. Zebrafish. 2013 Mar;10(1):1-14. PubMed PMID: 23590398.

94. Amatruda JF, Patton EE. Genetic models of cancer in zebrafish. International review of cell and molecular biology. 2008;271:1-34. PubMed PMID: 19081540.

95. Santoriello C, Zon LI. Hooked! Modeling human disease in zebrafish. The Journal of clinical investigation. 2012 Jul 2;122(7):2337-43. PubMed PMID: 22751109. Pubmed Central PMCID: 3386812.

96. Stoletov K, Klemke R. Catch of the day: zebrafish as a human cancer model. Oncogene. 2008 Jul 31;27(33):4509-20. PubMed PMID: 18372910.

97. Howe K, Clark MD, Torroja CF, Torrance J, Berthelot C, Muffato M, et al. The zebrafish reference genome sequence and its relationship to the human genome. Nature. 2013 Apr 25;496(7446):498-503. PubMed PMID: 23594743. Pubmed Central PMCID: 3703927.

98. Jaillon O, Aury JM, Brunet F, Petit JL, Stange-Thomann N, Mauceli E, et al. Genome duplication in the teleost fish Tetraodon nigroviridis reveals the early vertebrate proto-karyotype. Nature. 2004 Oct 21;431(7011):946-57. PubMed PMID: 15496914.

99. Postlethwait J, Amores A, Cresko W, Singer A, Yan YL. Subfunction partitioning, the teleost radiation and the annotation of the human genome. Trends in genetics : TIG. 2004 Oct;20(10):481-90. PubMed PMID: 15363902.

100. White R, Rose K, Zon L. Zebrafish cancer: the state of the art and the path forward. Nature reviews Cancer. 2013 Sep;13(9):624-36. PubMed PMID: 23969693.

101. Amatruda JF, Shepard JL, Stern HM, Zon LI. Zebrafish as a cancer model system. Cancer cell. 2002 Apr;1(3):229-31. PubMed PMID: 12086858.

102. Kimmel CB, Ballard WW, Kimmel SR, Ullmann B, Schilling TF. Stages of embryonic development of the zebrafish. Developmental dynamics : an official publication of the American Association of Anatomists. 1995 Jul;203(3):253-310. PubMed PMID: 8589427.

103. White RM, Sessa A, Burke C, Bowman T, LeBlanc J, Ceol C, et al. Transparent adult zebrafish as a tool for in vivo transplantation analysis. Cell stem cell. $2008 \mathrm{Feb}$ 7;2(2):183-9. PubMed PMID: 18371439. Pubmed Central PMCID: 2292119.

104. Eisen JS, Smith JC. Controlling morpholino experiments: don't stop making antisense. Development. 2008 May;135(10):1735-43. PubMed PMID: 18403413. 


\section{CHAPTER 1}

105. Perner B, Englert C, Bollig F. The Wilms tumor genes wt1a and wt1b control different steps during formation of the zebrafish pronephros. Developmental biology. $2007 \mathrm{Sep}$ 1;309(1):87-96. PubMed PMID: 17651719.

106. Drummond IA, Davidson AJ. Zebrafish kidney development. Methods in cell biology. 2010;100:233-60. PubMed PMID: 21111220.

107. Neugebauer JM, Amack JD, Peterson AG, Bisgrove BW, Yost HJ. FGF signalling during embryo development regulates cilia length in diverse epithelia. Nature. 2009 Apr 2;458(7238):651-4. PubMed PMID: 19242413.

108. Jaffe KM, Thiberge SY, Bisher ME, Burdine RD. Imaging cilia in zebrafish. Methods Cell Biol.97:415-35. PubMed PMID: 20719283.

109. Zhou W, Dai J, Attanasio M, Hildebrandt F. Nephrocystin-3 is required for ciliary function in zebrafish embryos. Am J Physiol Renal Physiol. Jul;299(1):F55-62. PubMed PMID: 20462968.

110. Sullivan-Brown J, Schottenfeld J, Okabe N, Hostetter CL, Serluca FC, Thiberge SY, et al. Zebrafish mutations affecting cilia motility share similar cystic phenotypes and suggest a mechanism of cyst formation that differs from pkd2 morphants. Developmental biology. 2008 Feb 15;314(2):261-75. PubMed PMID: 18178183. Pubmed Central PMCID: 2453220.

111. Simms RJ, Hynes AM, Eley L, Inglis D, Chaudhry B, Dawe HR, et al. Modelling a ciliopathy: Ahi1 knockdown in model systems reveals an essential role in brain, retinal, and renal development. Cellular and molecular life sciences : CMLS. 2012 Mar;69(6):993-1009. PubMed PMID: 21959375.

112. Ferrante MI, Romio L, Castro S, Collins JE, Goulding DA, Stemple DL, et al. Convergent extension movements and ciliary function are mediated by ofd1, a zebrafish orthologue of the human oral-facial-digital type 1 syndrome gene. Human molecular genetics. 2009 Jan 15;18(2):289-303. PubMed PMID: 18971206. Pubmed Central PMCID: 2638777.

113. Pinkus H, Coskey R, Burgess GH. Trichodiscoma. A benign tumor related to haarscheibe (hair disk). The Journal of investigative dermatology. 1974 Aug;63(2):212-8. PubMed PMID: 4843452.

114. Banik R, Lubach D. Skin tags: localization and frequencies according to sex and age. Dermatologica. 1987;174(4):180-3. PubMed PMID: 3582706.

115. Claessens T, Vernooij M, Luijten M, Coull BJ, Van Steensel MAM. What's new in BirtHogg-Dubé syndrome? Expert Review of Dermatology. 2012 Dec;7(6):521-8.

116. Vernooij M, Claessens T, Luijten M, van Steensel MA, Coull BJ. Birt-Hogg-Dube syndrome and the skin. Familial cancer. 2013 Sep;12(3):381-5. PubMed PMID: 23307118.

117. Vincent A, Farley M, Chan E, James WD. Birt-Hogg-Dube syndrome: a review of the literature and the differential diagnosis of firm facial papules. Journal of the American Academy of Dermatology. 2003 Oct;49(4):698-705. PubMed PMID: 14512919.

118. Starink TM, Kisch LS, Meijer CJ. Familial multiple trichodiscomas. A clinicopathologic study. Archives of dermatology. 1985 Jul;121(7):888-91. PubMed PMID: 4015134.

119. Starink TM, Houweling AC, van Doorn MB, Leter EM, Jaspars EH, van Moorselaar RJ, et al. Familial multiple discoid fibromas: a look-alike of Birt-Hogg-Dube syndrome not linked to the FLCN locus. Journal of the American Academy of Dermatology. 2012 Feb;66(2):259 e1-9. PubMed PMID: 21794948. 


\section{CHAPTER 2}

\section{Birt-Hogg-Dubé syndrome is a novel ciliopathy}

Human Molecular Genetics. 2013 Nov 1;22(21):4383-97.

Monique N. H. Luijten*, Sander G. Basten*, Tijs Claessens*, Marigje Vernooij, Claire L. Scott, Renske Janssen, Jennifer A. Easton, Miriam A. F. Kamps, Maaike Vreeburg, Jos L. V. Broers, Michel van Geel, Fred H. Menko, Richard P. Harbottle, Ravi K. Nookala, Andrew R. Tee, Stephen C. Land, Rachel Giles, Barry J. Coull, and Maurice A. M. van Steensel

* These authors contributed equally to the manuscript 


\section{Abstract}

Birt-Hogg-Dubé (BHD) syndrome is an autosomal dominant disorder where patients are predisposed to kidney cancer, lung and kidney cysts and benign skin tumors. BHD is caused by heterozygous mutations affecting Folliculin (FLCN), a conserved protein that is considered a tumor suppressor. Previous research has uncovered multiple roles for FLCN in cellular physiology, yet it remains unclear how these translate to BHD lesions.

Since BHD manifests hallmark characteristics of ciliopathies, we speculated that FLCN might also have a ciliary role. Our data indicate that FLCN localizes to motile and non-motile cilia, centrosomes and the mitotic spindle. Alteration of FLCN levels can cause changes to the onset of ciliogenesis, without abrogating it. In three dimensional culture, abnormal expression of FLCN disrupts polarized growth of kidney cells and deregulates canonical Wnt signalling. Our findings further suggest that BHD-causing FLCN mutants may retain partial functionality. Thus, several BHD symptoms may be due to abnormal levels of FLCN rather than its complete loss and accordingly, we show expression of mutant FLCN in a BHD-associated renal carcinoma. We propose that BHD is a novel ciliopathy, its symptoms at least partly due to abnormal ciliogenesis and canonical Wnt signalling. 


\section{Introduction}

Birt-Hogg-Dubé (BHD) syndrome (MIM \#135150) is a rare autosomal dominant disorder that was first described in 1975 by Hornstein and Knickenberg as a distinct disorder associated with intestinal polyps (1). Birt, Hogg and Dubé later reported the same disorder, but in association with medullary thyroid carcinoma (2). A clear association with kidney cancer, mostly of mixed clear cell/chromophobe histology (3), was recognized in 1999 (4) and has been extensively documented since. The prevalence of BHD is estimated at $1 / 200,000$ and the majority of papers published to date put the lifetime risk of developing renal cell carcinoma (RCC) in BHD patients at $\sim 30 \%$ (5). Our own, more recent data suggest a range of $16-20 \%$ (3). A roughly similar risk exists for pneumothorax, possibly due to basal lung cysts that are present to a varying degree in almost all BHD patients. About $80 \%$ of BHD patients will develop benign skin lesions called fibrofolliculomas (5), generally after the age of 35. An emerging aspect of the BHD phenotype is cyst formation in kidney, liver and the pancreas [Fig. 1, and (6)].BHD is caused by mostly truncating mutations in the gene coding for the protein FLCN (7), whose functions are largely unknown but which is considered a tumor suppressor $(8,9)$.FLCN is an ancient and highly conserved protein, with multiple orthologs present in fungi and animals. Previous research suggests that FLCN is a downstream target of both AMP-dependent protein kinase (AMPK) and mammalian Target of Rapamycin complex 1 (mTORC1) signalling (10). FLCN might also modulate mTORC1, but conflicting data obtained in cells and tissues that lack FLCN show both up- and down-regulation of mTORC1 activity $(9$, 11-13). We recently reported that the absence of FLCN causes aberrant hypoxiainducible factor 1 transcriptional activity and the Warburg effect, where FLCNdeficient cells favoured aerobic glycolysis over oxidative phosphorylation (14). Deregulation of TGF $\beta$ signalling in FLCN-deficient cells has also been reported, although the reports are contradictory on the nature of FLCN's involvement (15, 16). FLCN has recently been implicated in control of ribosomal RNA synthesis through an interaction with the protein RPT4 (17), a finding that might explain the aberrant transcriptional activity observed in a number of studies $(14,15)$.

The von Hippel-Lindau (VHL) and Tuberous Sclerosis Complex disease syndromes, predisposing to malignant and benign renal tumors, respectively, have previously been linked to impaired cilia function and cyst formation $(18,19)$. Since BHD patients also develop cysts in multiple organs such as kidney, liver and lungs, we hypothesized that FLCN might similarly have a functional role in primary cilia and that ciliary dysfunction could contribute to the BHD phenotype. Cilia are microtubule-based structures that are enveloped by a highly specialized membrane and protrude from the apical cell membrane. Cilia formation is restricted to cells that have exited the cell cycle, allowing the centrosome to differentiate into a basal body that forms the base of the cilium (20). Different ciliary subtypes have been described, of which primary (non-motile) cilia are the 
most common and are considered to have primarily sensory functions. Motile cilia are predominantly involved with generating fluid flow (21).

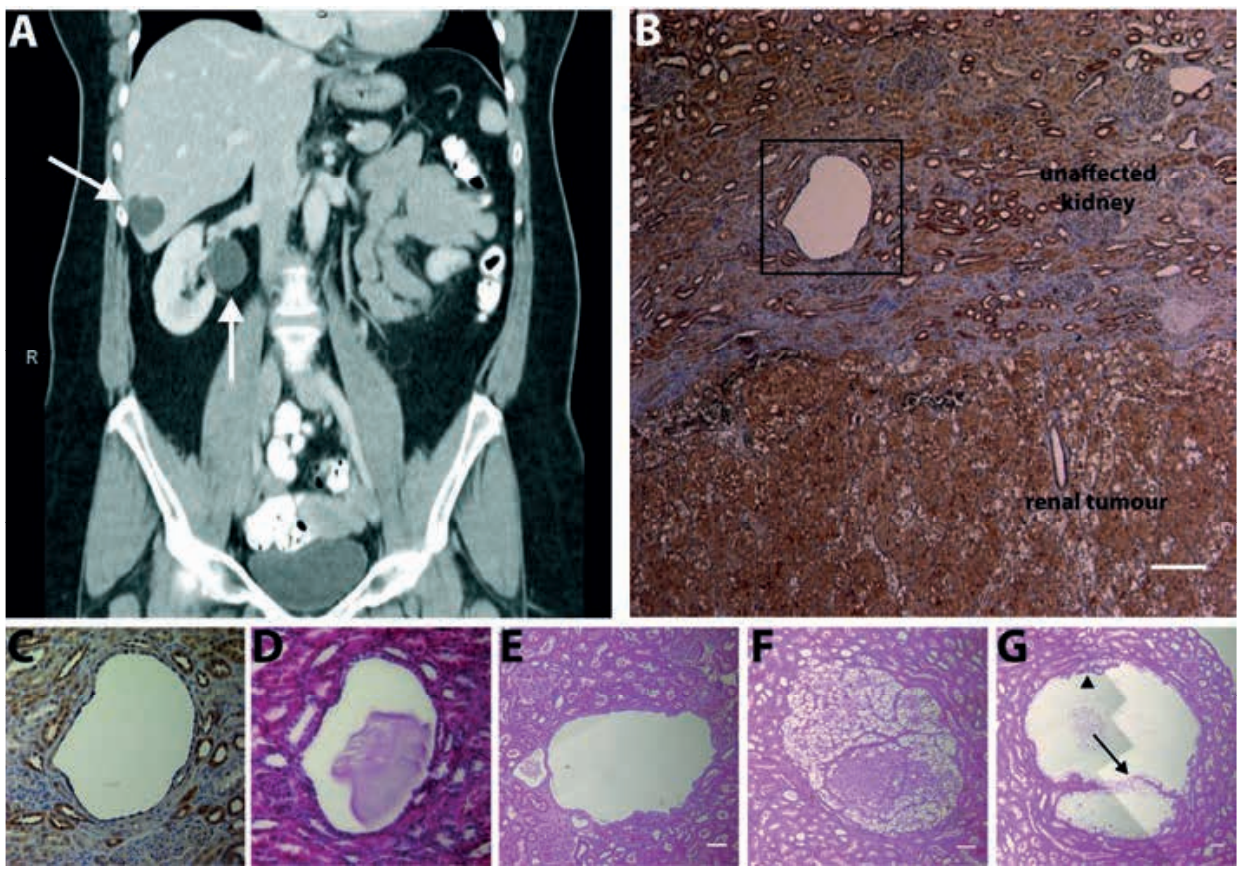

Figure 1. Birt-Hogg-Dubé syndrome is associated with development of renal cysts

(A) CT scan of a BHD patient. Coronal plane. Arrows indicate cysts in liver and kidney. (B) Paraffinembedded samples were obtained from a renal carcinoma from a BHD patient with a c.499C >T mutation (encoding pGln167X). Immunohistochemical staining with custom made C terminal FLCN antibody revealed FLCN around kidney tubules and within the tumour. Highlighted area around the kidney cyst is shown in figure 1C. Magnification 50x. Scale bar is $400 \mu \mathrm{m}$. (C) Magnification highlighted area figure 1B. Magnification 400x (D) H\&E stain of highlighted area figure 1B. Magnification 400x (E) H\&E stain (composite of 3 images) of a cyst from Nihon Rat kidney tissue. H\&E, Magnification 50x Scale bar is $100 \mu \mathrm{m}$. (F) H\&E stain (composite of 4 images) of a tumour from Nihon Rat kidney tissue. Magnification 50x Scale bar is $100 \mu \mathrm{m}$. (G) H\&E stain (composite of 6 images) of a cyst from Nihon Rat kidney tissue. There are clear cysts containing two distinct populations of cells. The first population with cuboidal morphology (arrow), and the second with an eosinophilic cytoplasm that protruded to into the cyst lumen (arrowhead). Magnification 50x. Scale bar is $100 \mu \mathrm{m}$.

Dysfunction of (primary) cilia is a universal cause of the ciliopathies, which are characterized by pathophysiology associated with changes in the morphology and/or number of cilia. The large group of disorders classified as ciliopathies is particularly (but not exclusively) associated with cyst formation in several organs, including kidneys $(22,23)$. In recent years, it has become apparent that cilia act as a master switch essential to maintain normal kidney morphology. The mechanisms involved are far from resolved, but many links have been made between ciliary signalling and planar cell polarity (PCP), the mechanism that defines the cellular orientation in tissue morphology (24). Defective PCP can cause renal tubule 
elongation defects, which are believed to underlie cystic growth initiation (25). Cysts may represent a potential pre-tumorigenic stage (26) and ciliary dysfunction is increasingly implicated in the pathogenesis of several cancer types, including renal carcinoma (27), melanoma (28), pancreatic ductal adenocarcinoma (29) and ovarian cancer $(30,31)$.

We set out to test our hypothesis by examining FLCN's subcellular localization in various cellular models and its influence on ciliary number, morphology and function. Our data suggest that BHD syndrome can be considered a novel ciliopathy, and that FLCN has a role in regulating cellular morphology and PCP, possibly through interaction with Wnt signalling. We also provide evidence that BHD patient derived FLCN mutants retain functionality, which has important implications for understanding and treating BHD manifestations. If therapeutic or preventive approaches to BHD-associated pathology are to be developed, FLCN's functional role(s) must ultimately be elucidated.

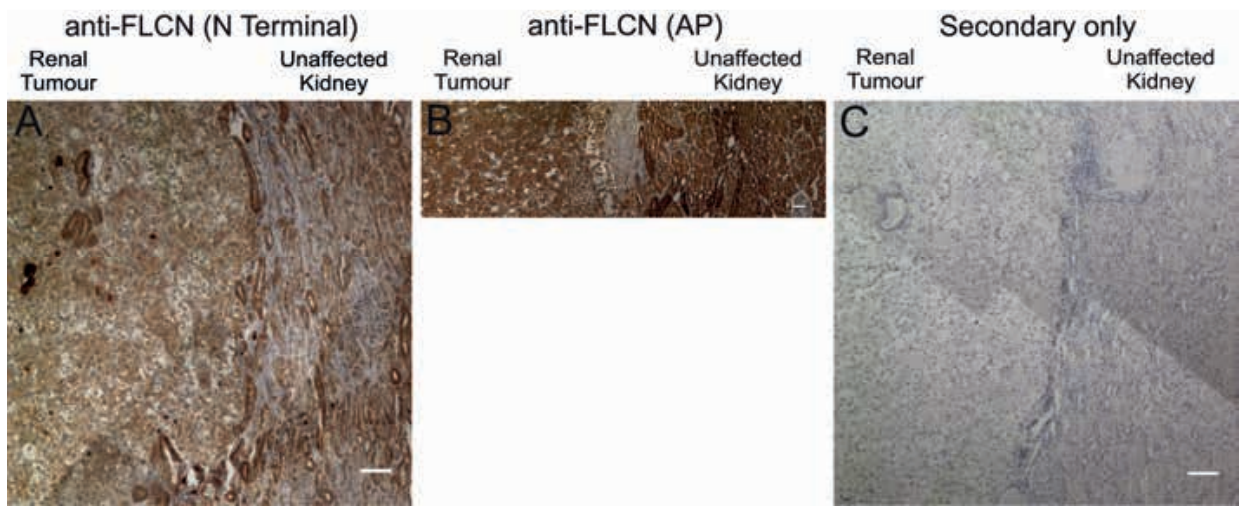

Supplementary figure S1. Additional IHC staining for Paraffin-embedded BHD renal carcinoma

Paraffin-embedded samples were obtained from a renal carcinoma from a BHD patient with a c.499C $>\mathrm{T}$ mutation (encoding pGln167X).

(A) Immunohistochemical staining (composite of 6 images) with custom made $\mathrm{N}$ terminal FLCN antibody revealed FLCN around kidney tubules and within the tumour. Magnification 50x. Scale bar is $100 \mu \mathrm{m}$. (B) Immunohistochemical staining (composite of 10 images) with FLCN AP antibody revealed FLCN around kidney tubules and within the tumour. Magnification 50x. Scale bar is $100 \mu \mathrm{m}$. (C) Control immunohistochemical staining (composite of 6 images) with secondary antibody only. Magnification $50 \mathrm{x}$. Scale bar is $100 \mu \mathrm{m}$. 


\section{Results}

BHD syndrome is associated with development of renal cysts

BHD patients develop cysts in multiple internal organs such as kidney and liver (Fig. 1A). Using a custom-made antibody directed against the C-terminus of FLCN, we stained kidney tumor material derived from a BHD patient with a c.499C>T mutation (that encodes for a truncated FLCN mutant, pGln167X) (Fig. 1B). Hence, this antibody will only detect the wild-type protein, not this mutant form. The kidney sample contains both unaffected kidney (Fig. 1B, upper section) and clearly demarcated renal tumor material with mixed chromophobe/clear cell histology (Fig. 1B, lower section). We observed strong expression within the normal kidney and clear staining for FLCN around the kidney tubules (Fig. 1B and C and Supplementary Material, Fig. S1). We also noted a cystic area devoid of cells (Fig. 1B-D), which represents a small kidney cyst. Previously, we determined that the remaining FLCN allele in this tumor contains a second hit of a 15 base pair deletion, predicted to result in the deletion of exon 6 (32). Within the tumor mass, we still observe staining for FLCN (Fig. 1B, lower section), suggesting the presence of a mutant form of the protein in this particular tumor. Next we examined kidney material obtained from the Nihon rat BHD animal model (33). The Nihon rat has a naturally occurring mutation in Flcn that is predicted to result in a severely truncated Flcn protein. Upon examination of the kidney, multiple cysts (Fig. 1E) as well as cancerous lesions were evident (Fig. 1F). Of note, the cysts contained two populations of cells - one population of clear cells with a cuboidal morphology that lined the cyst (Fig. 1G, see arrow) and another population with eosinophilic cytoplasm that grew into the cyst lumen (Fig. 1G, see arrowhead).

Thus, both humans and rats with BHD syndrome develop kidney cysts, highlighting that BHD might be a member of a larger group of disorders called ciliopathies, of which cysts in various organs, particularly the kidneys, are hallmark lesions. Ciliopathies are associated with cystic disease linked to changes in the number and/or shape of cilia (23). As ciliopathy proteins often localize to the cilium (although this is not always a prerequisite) (34), we hypothesized that FLCN might localize to cilia and proceeded to examine whether FLCN is a ciliary protein.

\section{FLCN localizes to a number of microtubule-based structures}

We determined the localization of endogenous FLCN in several different cell types that form primary cilia. To rule out the possibility of a non-specific staining, we utilized several different FLCN antibodies. In all cell lines and with all antibodies used, we observed a clear and specific staining of anti-FLCN antibodies to primary cilia (Fig. 2A and Supplementary Material, Fig. S2). To further rule out the possibility of a non-specific antibody stain, we stably expressed EGFP-tagged FLCN (hereafter referred to as EGFP-FLCN WT) in type II Madin-Darby Canine 


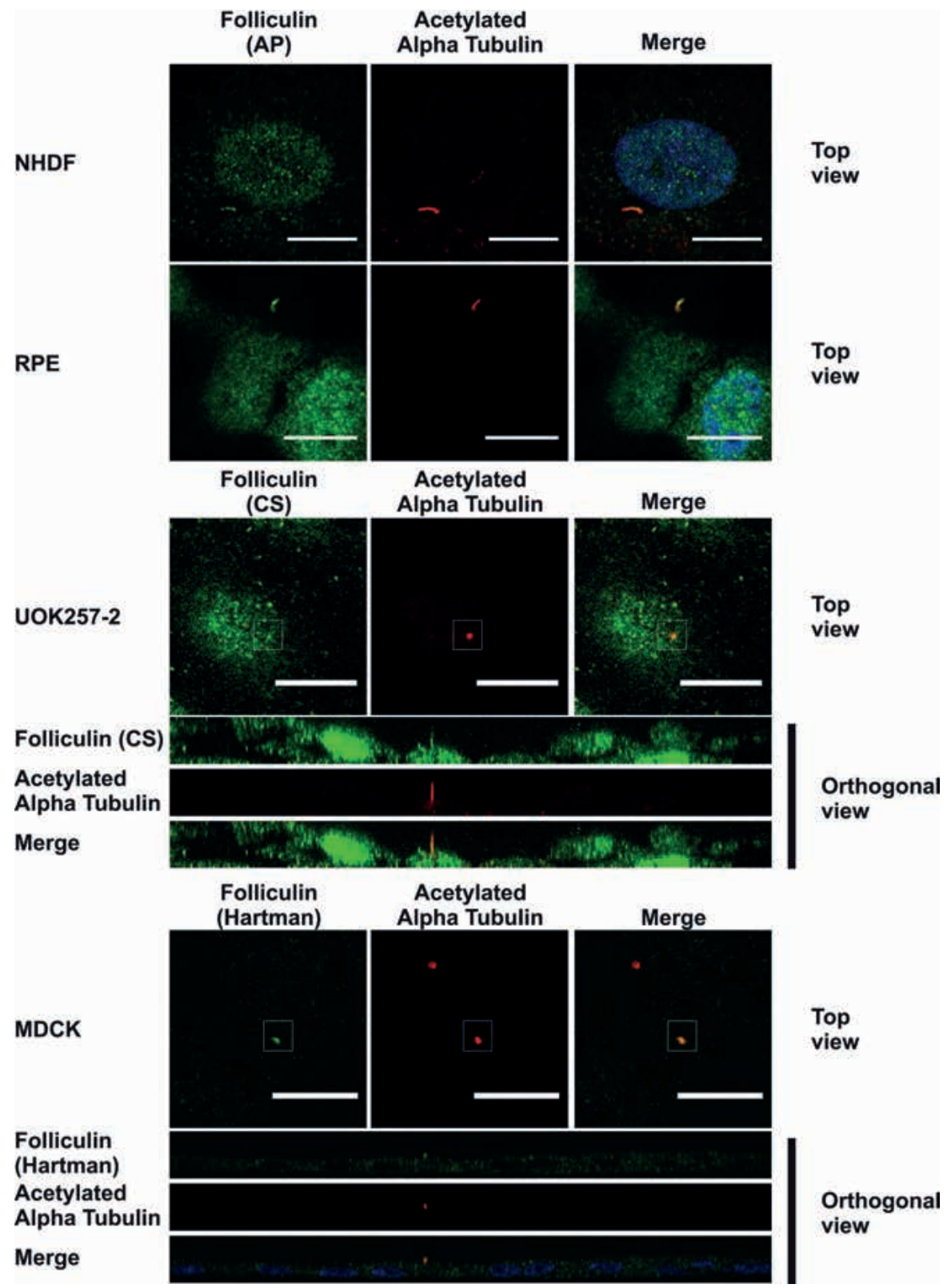

Supplementary figure S2. FLCN is detected at the primary cilia of various cell types using various antibodies

Normal human dermal fibroblasts (NHDF), retinal pigmented epithelial cells (RPE), UOK257-2 and MDCK cells were allowed to ciliate, fixed and stained for acetylated alpha tubulin to mark the ciliary axoneme (red) and for endogenous FLCN (green) using indicated FLCN antibodies. Nuclei were stained with DAPI (blue). Cilia in white boxes are shown in orthogonal views below. Scale bar is $10 \mu \mathrm{m}$. 

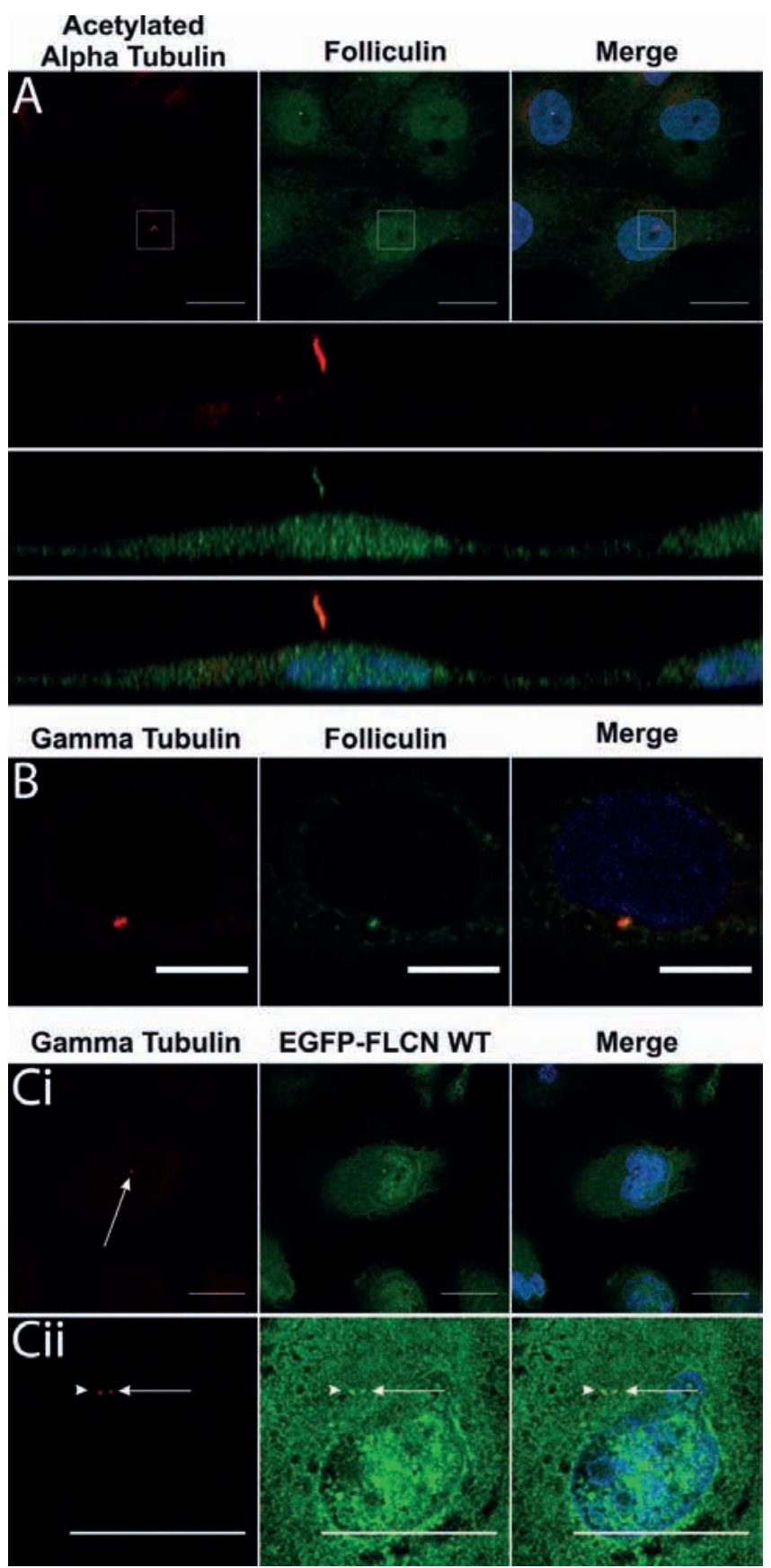

Figure 2. FLCN is a constitutive ciliary, basal body and centrosomal protein

In this figure all endogenous FLCN stains were obtained using the FLCN AP antibody. (A) FLCN localises to primary cilia HK-2 cells serum starved for 48 hours were fixed and stained for endogenous FLCN (green) and acetylated alpha tubulin (red). Nuclei were stained with DAPI (blue). Cilium in the white box is shown in orthogonal view below. Scale bar is $10 \mu \mathrm{m}$. (B) EGFP-FLCN WT localises to the centrosome MDCK cells stably expressing EGFPtagged FLCN WT were fixed and counterstained with antigamma-tubulin (red) to mark the centrosomes (indicated by arrows). EGFP-FLCN WT localises at centrosome before (B i scale bar is $20 \mu \mathrm{m}$ ) and after centrosome duplication (B ii scale bar is $10 \mu \mathrm{m})$. Nuclei are stained with DAPI (blue). (C) Endogenous FLCN localises to the centrosome IMCD3 cells were fixed and stained for gamma-tubulin (red) and endogenous FLCN (green). Nuclei are stained with DAPI (blue). Scale bar is $10 \mu \mathrm{m}$ 

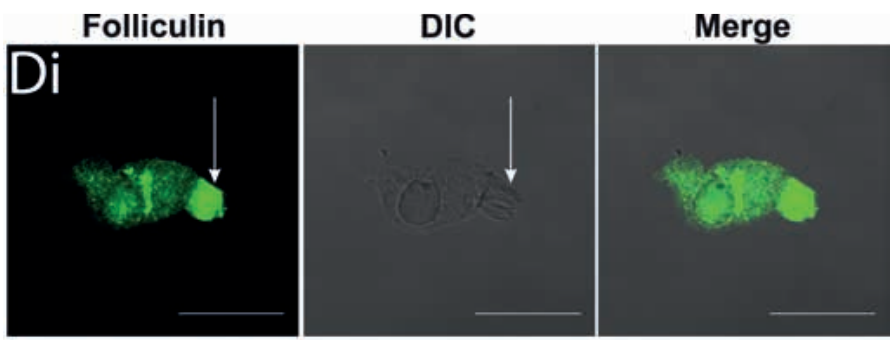

Acetylated

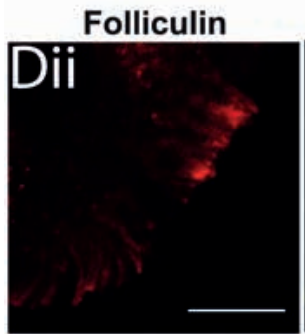
Alpha Tubulin
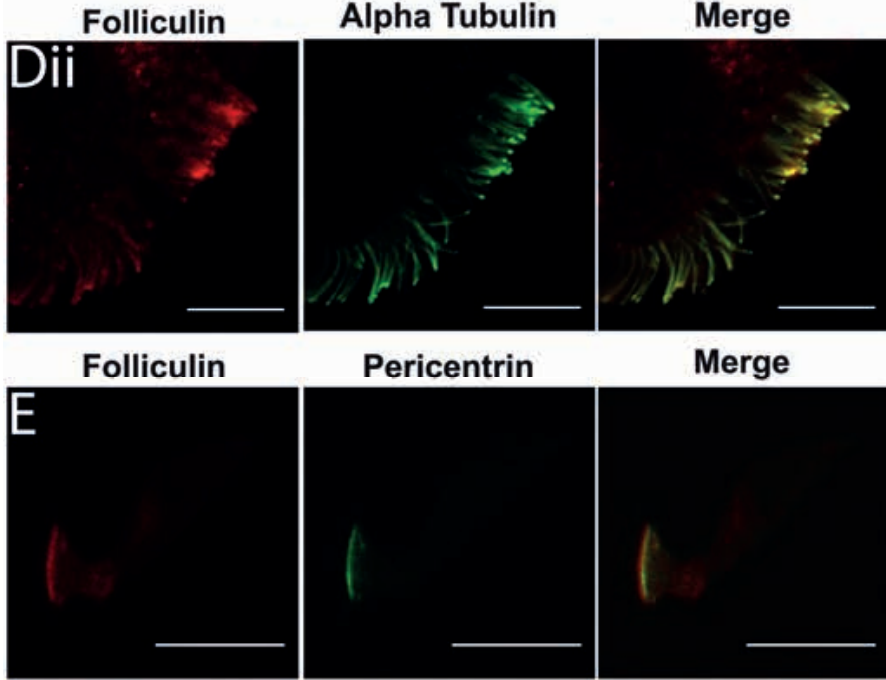

\section{Pericentrin}
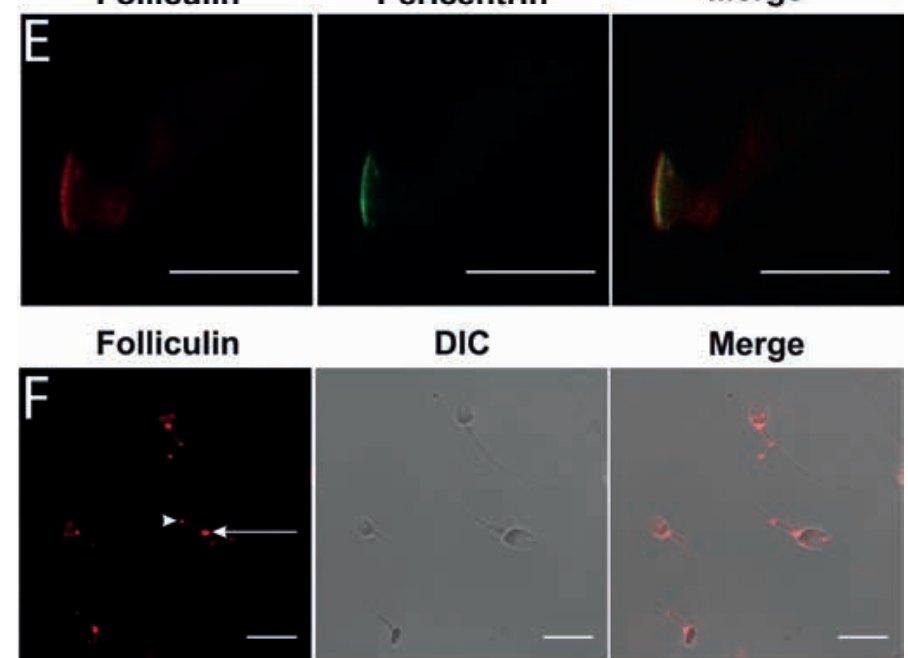

\section{Acetylated}

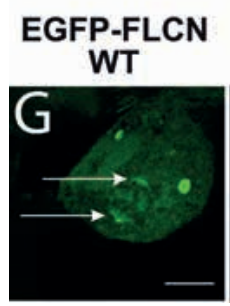

Alpha Tubulin

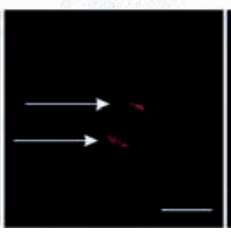

DIC

Merge

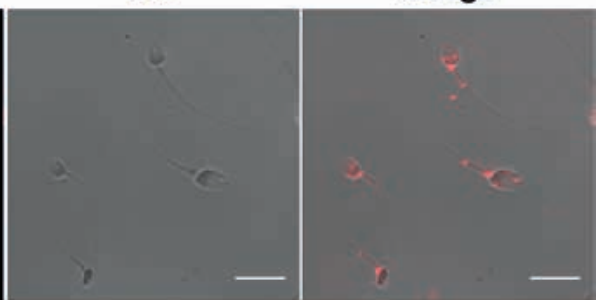

DAPI

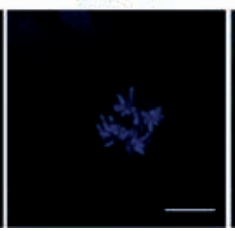

Figure 2. FLCN is a constitutive ciliary, basal body and centrosomal protein (continued)

(D) FLCN localises to motile cilia (i) Single stain. Primary Human Nasal Epithelial (HNE) cells were fixed and stained for endogenous FLCN (green). Middle panel is DIC image for visualisation of cilia (indicated by the arrow). Scale bar is $20 \mu \mathrm{m}$. (ii) Double stain. HNE cells were fixed and stained for endogenous FLCN (red) and acetylated alpha tubulin (green). Scale bar is $10 \mu \mathrm{m}$. (E) FLCN localises to the basal body HNE cells were fixed and stained for endogenous FLCN (red) and pericentrin (green) to mark the base plate. Scale bar is $20 \mu \mathrm{m}$. (F) In human sperm endogenous FLCN localises to the centriole and annulus Human sperm cells were fixed and stained for endogenous FLCN (red). Note the staining at the centriole (arrowhead) and annulus (arrow). Scale bar is $10 \mu \mathrm{m}$. (G) FLCN localises to the mitotic spindle HK-2 cells overexpressing EGFPFLCN WT were fixed and counterstained with acetylated alpha tubulin (red) to mark the mitotic spindles (indicated by arrows) and DAPI (blue). Scale bar is $20 \mu \mathrm{m}$. 
Kidney (MDCK) cells. In these cells, we observed EGFP-tagged FLCN localizing to cilia (Supplementary Material, Fig. S4A). In line with previous data, we observed FLCN localization throughout the cytoplasm and in the nucleus $(10,35)$. Interestingly, we noted that anti-FLCN antibody also localized to 1 or 2 discrete foci within the cell. We subsequently stained with the centrosome marker gammatubulin, and found co-localization, indicating that FLCN is a centrosomal protein (Fig. 2B). We confirmed these results in the stable cell line, where EGFP-FLCN WT (in addition to cilia) localizes at 1 or 2 discrete foci, indicating that after centrosome duplication FLCN localizes to both the centrosomes (Fig. 2C). Next we considered the possibility that FLCN might be a universal constituent of both primary and motile cilia and their respective basal bodies. In primary human nasal epithelial (HNE) cells that contain beating motile cilia, we observed localization of FLCN to the cilia (Fig. 2D) and at the base plate, which is the basal body equivalent for motile cilia (Fig. 2E). In Drosophila melanogaster, BHD-/- males exhibit a fertility defect (36). We therefore asked whether FLCN is present in sperm flagellae or their associated structures. The sperm flagellum is structurally identical to motile cilia, although its beating pattern is different (37). Some ciliopathies are associated with sperm motility defects (38). As shown in Figure 2F, in human spermatozoa we observed clear staining of FLCN to the centriole (Fig. 2F, arrow) and annulus (Fig. 2F, arrowhead). The latter structure is equivalent to the ciliary diffusion barrier for non-motile cilia (37). Faint flagellar staining can also be observed, but due to the low level of staining it is not clear whether this is an artefact or genuine localization of FLCN in the flagellum.

Finally, several known ciliary proteins such as IFT88, LKB1 and VHL (39-41) are known to localize to the mitotic spindle. To see if this is the case for FLCN, we examined mitotic cells expressing EGFP-FLCN WT and indeed, we observed the EGFP-tagged FLCN protein localizing to the mitotic spindle (Fig. 2G).

\section{Disease-associated mutations of FLCN do not affect subcellular localization}

Having observed full-length FLCN localizing to cilia, the basal body and the centrosome, we reasoned that disease-associated mutations might affect FLCN's subcellular localization. Therefore, we expressed EGFP-tagged versions of several disease causing FLCN mutations in the immortalized proximal kidney tubule cell line HK-2 (42). The tagged versions of missense mutations p.His255Arg (H255R) and p.Lys508Arg (K508R) could be visualized at the centrosome (Supplementary Material, Fig. S3 and Supplementary Material, Table S1). Next, we examined EGFPtagged patient mutations that result in C-terminal deletions, p.Tyr463Stop (Y463X), and the most common mutation, p.His429ProfsX27. Again, both of these truncating mutants localize to the centrosomes. These observations suggest that BHD-associated truncating FLCN mutations do not prevent centrosomal localization. 
Unfortunately, due to consistently low expression levels and a deleterious effect on ciliation, localization of the H255R, Y463X and p.His429ProfsX27 mutant FLCN proteins to cilia and the mitotic spindle could not be determined. However, we successfully visualized EGFP- FLCN K508R in the cilia (Supplementary Material, Fig.S4A)and at the mitotic spindle (Supplementary Material, Fig. S4B). Thus, the localization of the K508R mutant seems comparable with that of the wild-type protein.

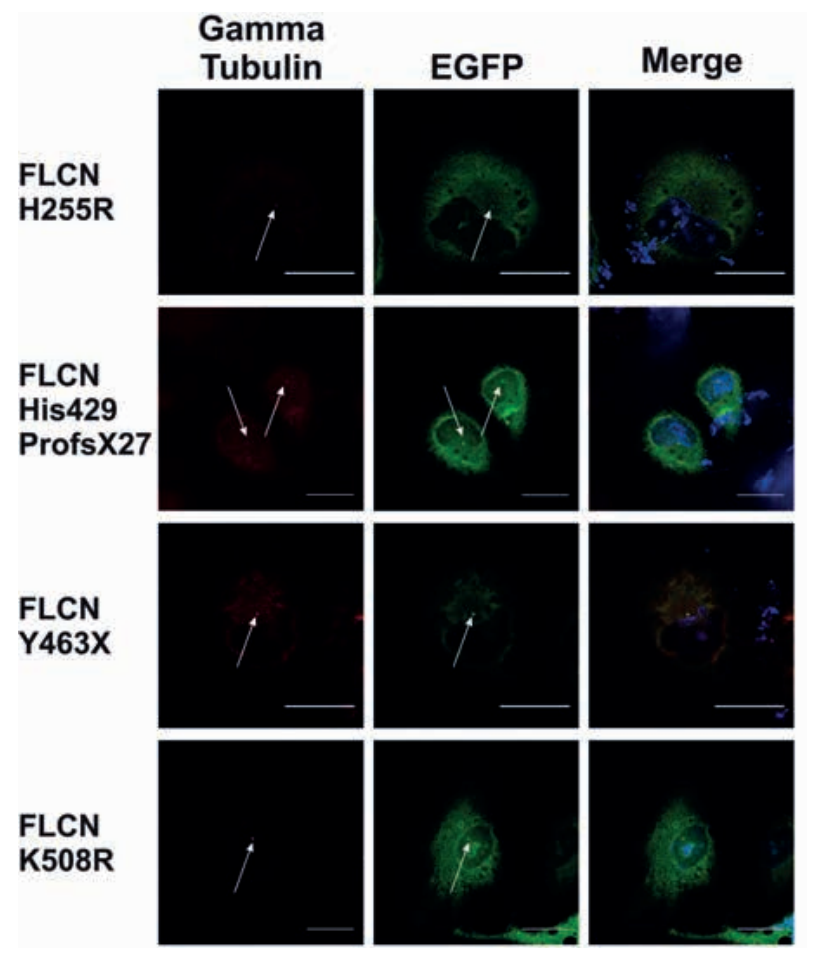

Supplementary figure S3. Diseaseassociated mutations of FLCN do not affect FLCN localisation to the centrosome

HK-2 cells were transiently transfected with the indicated EGFP tagged disease-causing mutations. 24 hours post transfection cells were fixed and counterstained with gammatubulin (red) to mark the centrosome (indicated by white arrows). Nuclei are stained with DAPI (blue). Scale bar is $20 \mu \mathrm{m}$.

Supplementary Table 1. Localisation of EGFP tagged constructs to the centrosome

\begin{tabular}{ll}
\hline Construct & Centrosomal localisation \\
\hline EGFP & No \\
EGFP-FLCN Wild Type & Yes \\
EGFP-FLCN His255Arg (H255R) & Yes \\
EGFP-FLCN Lys508Arg (K508R) & Yes \\
EGFP-FLCN Tyr463 Stop (Y463X) & Yes \\
EGFP-FLCN His429 ProfsX27 & Yes \\
\hline
\end{tabular}




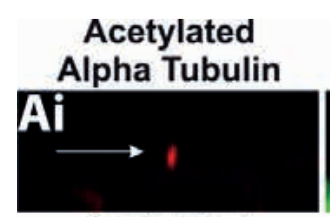

Acetylated Alpha Tubulin

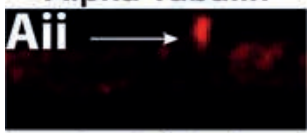

Acetylated Alpha Tubulin

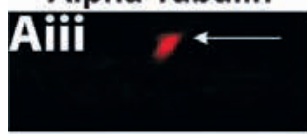

Acetylated Alpha Tubulin

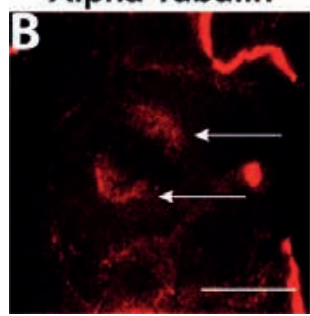

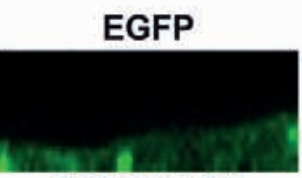

EGFP-FLCN

WT

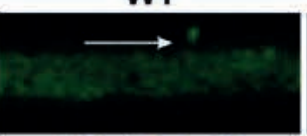

EGFP-FLCN

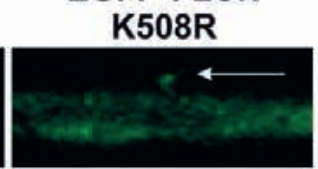

EGFP-FLCN K508R

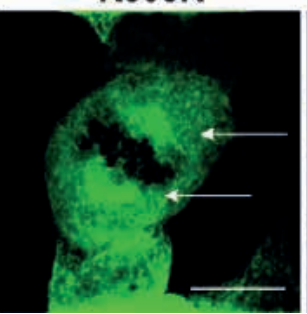

DAPI

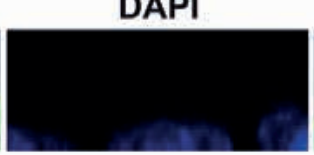

DAPI

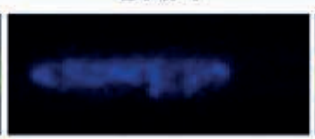

DAPI

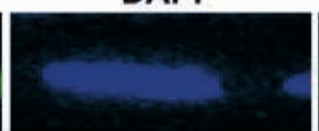

DAPI

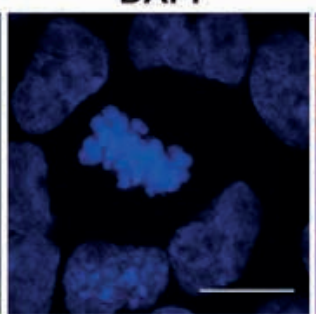

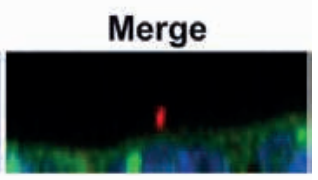

Merge

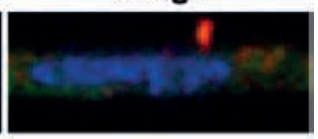

Merge

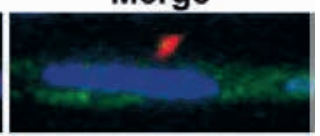

Merge

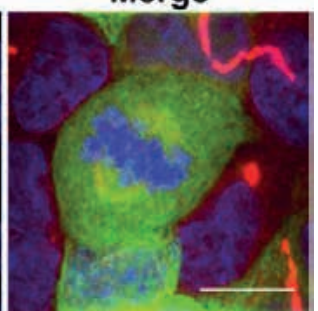

Supplementary figure S4. The disease-associated mutation K508R of FLCN does not affect FLCN localisation to the cilia or mitotic spindle

(A) Orthogonal projections of MDCK cells stably overexpressing EGFP (i), EGFP-FLCN WT (ii) or EGFPFLCN K508R (iii) maintained at confluency for 5 days, fixed and stained for acetylated alpha tubulin to mark the ciliary axoneme (red). Nuclei were stained with DAPI (blue).

(B) MDCK cells overexpressing EGFP-FLCN K508R were fixed and counterstained with acetylated alpha tubulin (red) to mark the mitotic spindles (indicated by arrows) and DAPI (blue). Scale bar is $10 \mu \mathrm{m}$.

\section{The BHD-derived renal cancer cell line UOK257 shows abnormal ciliation}

As loss of cilia is a frequent occurrence in kidney cancer (27), we were interested whether BHD-derived renal cancer cells can form cilia. UOK257 is a BHD-derived kidney tumor cell line carrying the most common germline FLCN mutation, c.1285dupC (43), resulting in the FLCN truncation p.His429ProfsX27. Whereas BHD patients are heterozygotes, and still have one wild-type allele for the FLCN gene, the UOK257 cell line used in this study has been reported to have lost the wild-type allele and consequently only possesses the mutant copy (43).The presumed lack of functional FLCN might impair ciliogenesis. To test this idea, we compared the ability of UOK257 and UOK257-2 [UOK257 cells with re-expression of FLCN (10)] to ciliate. Neither cell line ciliated upon serum starvation, however, cilia were evident in both cell lines when left at full confluence for several days (Fig. 3A). Interestingly, we noted that after 6 days, confluent FLCN-rescued UOK257-2 cells exhibited fewer cilia than UOK257 cells. When we increased this 
time to 10 days, this difference between the cell lines was no longer evident (Fig. $3 \mathrm{~A}$ ), suggesting that overexpression of FLCN may impede formation of primary cilia. The length of the cilia observed in these UOK257 cells $(6.3 \pm 2.5 \mu \mathrm{m} \mathrm{n}=74)$ was not significantly different to that observed in our control HK-2 cell line (7.0 $\mu \mathrm{m} \pm 3.2 \mu \mathrm{m} n=147$ ).

Given the apparent differences we observed in ciliation between the UOK cell lines, we then sought to confirm their identity by sequencing the FLCN locus. Upon genomic sequencing of all of the FLCN exons using intronic primers, the only FLCN mutation detected was His429ProfsX27. However, it was also evident that a low level of the wild-type allele was present in the cells (Fig. 3B). This finding was subsequently confirmed by both immunofluorescence and western blotting experiments (see Supplementary Material, Fig. S5). Thus, we conclude that UOK257 cells are not FLCN null, as originally reported, but rather still express fulllength, wild-type FLCN.

A
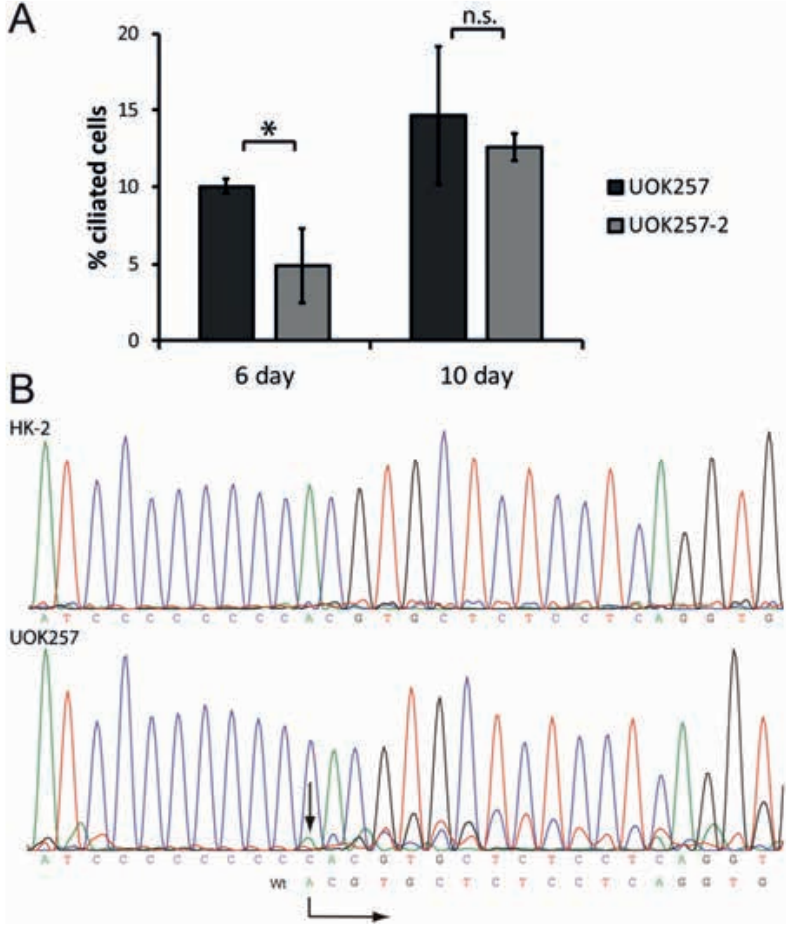

Figure 3. The BHD-derived renal cancer cell line UOK257 shows abnormal ciliation

(A) UOK257 and UOK257-2 cells were maintained at confluence for indicated amount of days, fixed and stained with acetylated alpha tubulin to mark the ciliary axoneme and pericentrin to mark the ciliary base. Nuclei were stained with DAPI and the percentage of ciliated cells was counted using ImageJ. After 6 days of confluence UOK257 had a significantly reduced number of cilia compared to UOK257-2 ( $\mathrm{p}=0.023)$. After 10 days of confluence both cell lines have a similar number of cilia $(p=0.422)$. Error bars represent standard error of the mean. A minimum of 365 cells were counted per cell line per time point. ${ }^{*}$ is $\mathrm{p}<0.05$

(B) Sequencing of genomic DNA isolated from HK-2 and UOK257 cells showing presence of the wild-type allele (starting sequence indicated by the arrow). 


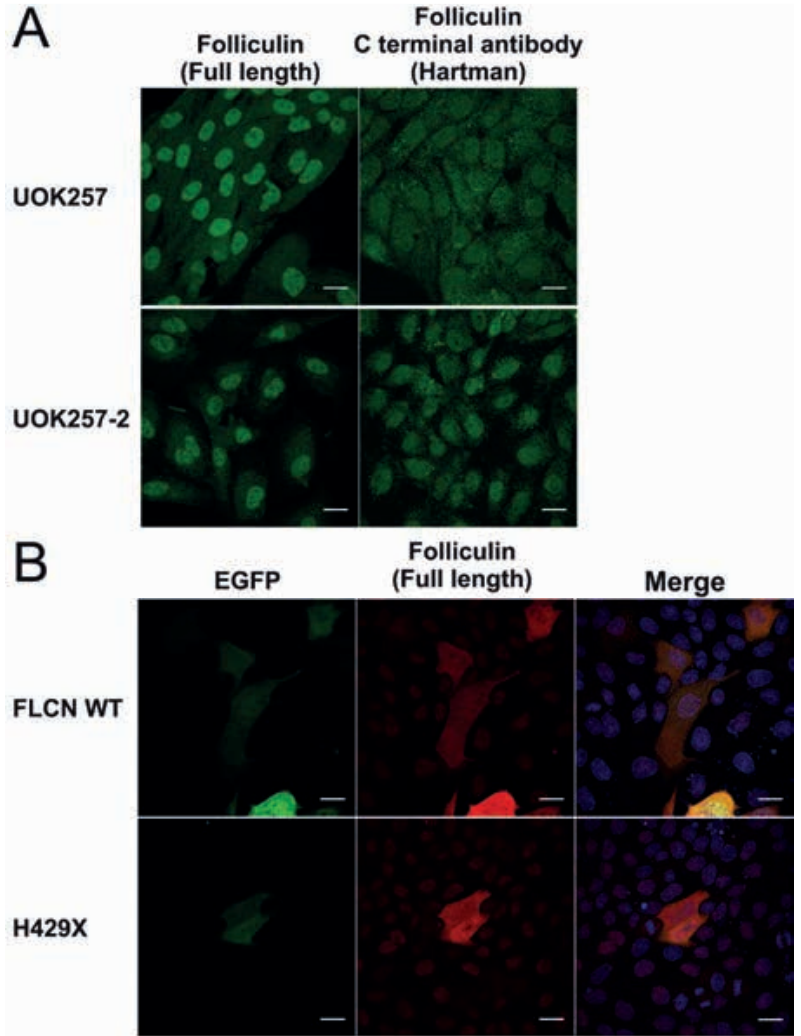

Folliculin

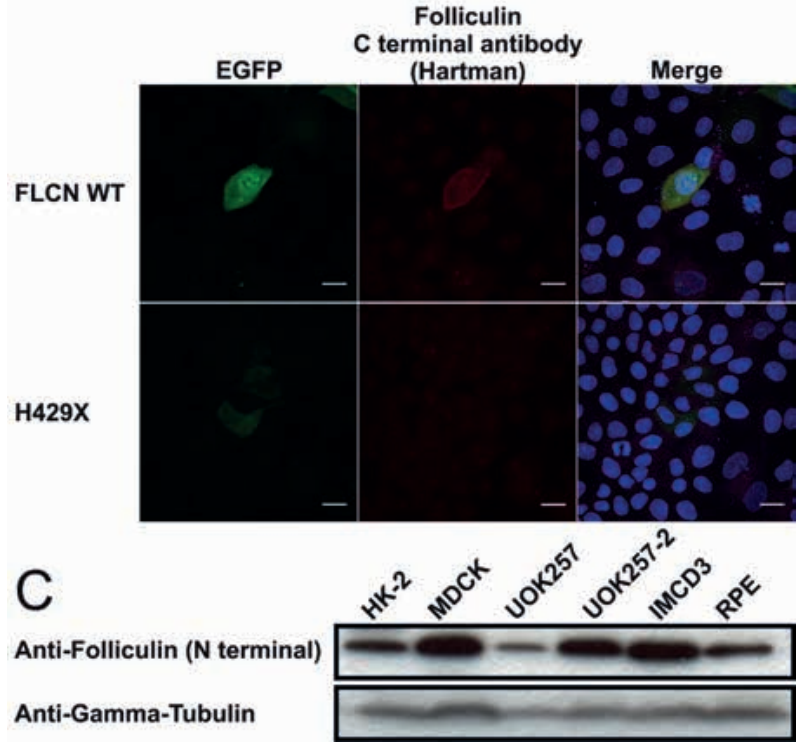

Supplementary figure S5. UOK257 cells express wild-type FLCN.

(A) UOK257 and UOK257-2 cells were fixed and stained for endogenous FLCN (green) with indicated antibodies. Scale bar is $20 \mu \mathrm{m}$.

(B) MDCK cells transiently transfected with EGFP-FLCN WT or the disease-causing variant EGFP-FLCN p.His429ProfsX27 were fixed and stained with antibodies (red) against fulllength FLCN (top panel) and the C-terminus of FLCN (bottom panel). Nuclei were stained with DAPI (blue). Scale bar is $20 \mu \mathrm{m}$.

(C) Western blot on $50 \mu \mathrm{g}$ of whole cell lysate of indicated cell types probed with custom made N-terminal FLCN antibody. Gamma tubulin was used as a loading control. 


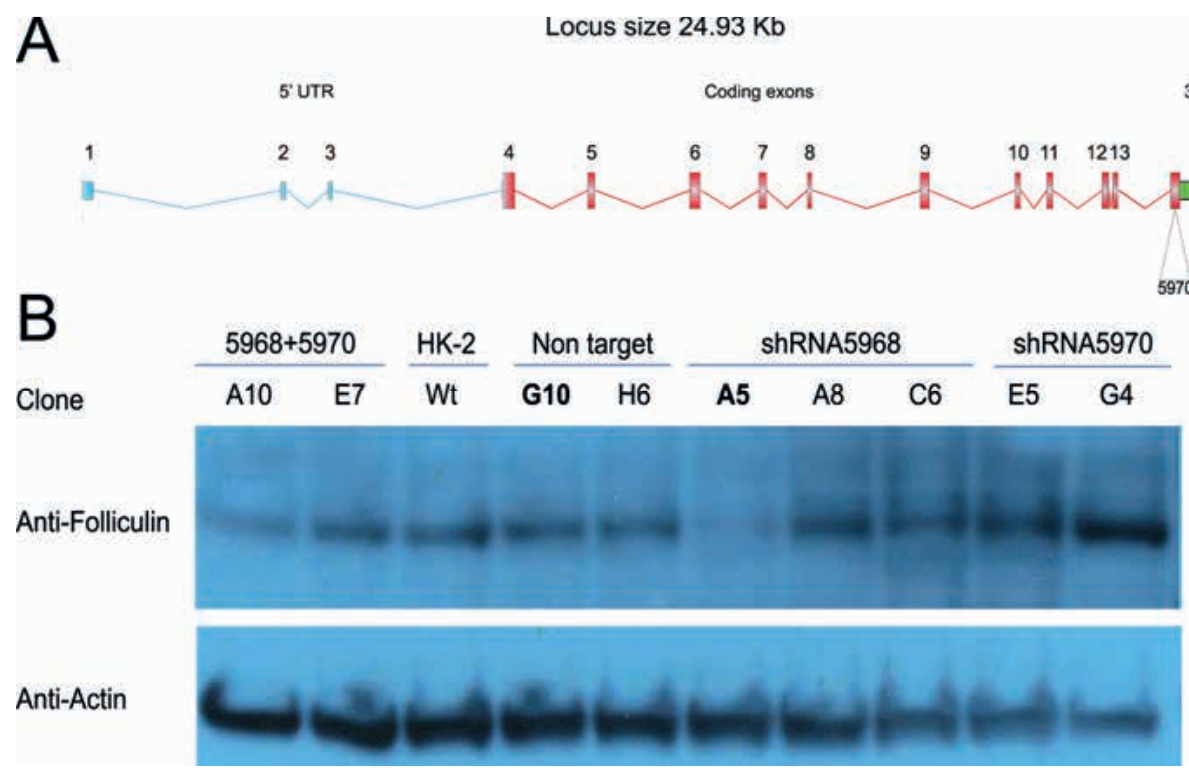

Supplementary figure S6. Knockdown of FLCN using shRNA in HK-2 cells

(A) Schematic representation of human FLCN locus. The 3'UTR is marked in blue, the coding exons in red and the 5' UTR in green.

The sequence of FLCN shRNA5968 corresponds to bp 775 - 795 of the 3'UTR. (GCTGCTTTCAACATTTACGTTCTCGAGAACGTAAATGTTGAAAGCAGC)

The sequence of FLCN shRNA5970 corresponds to bp 1677 -1697 of exon 14 of the coding sequence. (GACCTACAAGTCACACCTCATCTCGAGATGAGGTGTGACTTGTAGGTC)

(B) Western blot on $20 \mu \mathrm{g}$ of whole cell lysate from HK-2 cells stably transfected with shRNA FLCN shRNA5968, shRNA FLCN shRNA5970, both FLCN shRNA's (shRNA5968 and shRNA5970) or the nontarget shRNA control. The membrane was probed with FLCN AP antibody and actin was used as a loading control. FLCN knockdown HK-2 clone A5 and FLCN knockdown non-target control HK-2 clone G10 were selected. 
Knockdown of FLCN in human kidney cells delays ciliogenesis

Considering that UOK257 cells express a lower level of full length FLCN than seen in HK-2 cells (Supplementary Material, Fig. S5C), we wondered if the ciliary phenotype observed in these cells resulted from reduced levels of wild-type FLCN. Therefore, we generated stable knockdowns of FLCN in HK-2 cells (see Supplementary Material, Fig. S6). Subsequently, we selected FLCN knockdown HK2 clone A5 (hereafter referred to as FLCN KD, estimated 50\% knockdown) and the scrambled control HK-2 clone G10 (hereafter referred to as NT; Fig. 4A). Knockdown of FLCN did not alter cell morphology or growth rate (data not shown). Cells were plated at low density and subsequently serum-starved for 48, 72, 96 and $120 \mathrm{~h}$ to initiate ciliogenesis. As seen in Figure 4B, at 48 and $72 \mathrm{~h}$, the FLCN KD cell line showed a significant reduction in number of ciliated cells compared with the NT control cells. Knockdown or knockout of other ciliary proteins can also cause changes in the length of cilia (20). Cilia in the HK-2 FLCN KD cells showed a slight but significant reduction in length versus the NT control cell line after $48 \mathrm{~h}$ of serum starvation. This was not evident after $72 \mathrm{~h}$ of serum starvation (Fig. 4C). At the later time points of 96 and $120 \mathrm{~h}$, all cell lines exhibited more cilia, and no significant difference in the number of ciliated cells between FLCN KD and NT control was evident (Fig. 4C). Collectively, these data suggest that ciliogenesis is delayed rather than inhibited upon FLCN knockdown.

Figure 4. Knockdown and exogenous expression of FLCN affects ciliogenesis in kidney cells

(A) Western blot of knockdown and control cells

Western blot on $30 \mu \mathrm{g}$ of whole cell lysate showing stable knockdown of FLCN using shRNA in HK-2 clone FLCN KD compared to wild-type HK-2 and HK-2 with scrambled control shRNA clone NT, (also see supplementary figure S6). Membrane was probed with FLCN AP antibody. Actin was used as a loading control.

(B) Measurement of ciliogenesis in FLCN knockdown versus non target control

HK-2 FLCN KD and NT cells were serum starved for the indicated amount of hours, fixed and stained with acetylated alpha tubulin to mark the ciliary axoneme and pericentrin to mark the ciliary base. Nuclei were stained with DAPI and the percentage of ciliated cells and cilium length was measured using ImageJ. After $48(\mathrm{p}<0.001)$ and $72(\mathrm{p}<0.001)$ hours of serum starvation, HK-2 FLCN KD cells showed a statistically significant reduction in the percentage of ciliated cells compared to NT cells. After $96(p=0.915)$ and $120(p=0.067)$ hours of serum starvation there was no difference in the percentage of ciliated cells between the two cell lines. Error bars represent standard error of the mean. Average of 350 cells counted per cell line per time point. * is $\mathrm{p}<0.05$

(C) Measurement of cilia length in FLCN knockdown versus non-target control

Average cilium length of HK-2 FLCN KD cells $(n=40)$ was significantly decreased compared to cilia of NT cells $(n=124)$ after 48 hours of serum starvation $(p=0.001)$. After 72 hours of serum starvation no difference in cilia length was observed between HK-2 FLCN KD $(n=24)$ and NT cells $(n=55)(p=0.55)$. Error bars represent standard error of the mean. * is $\mathrm{p}<0.05$ 
A

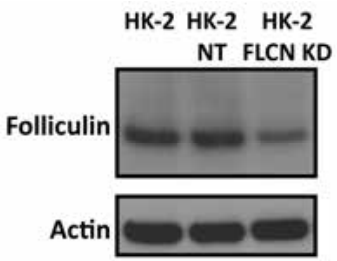

B

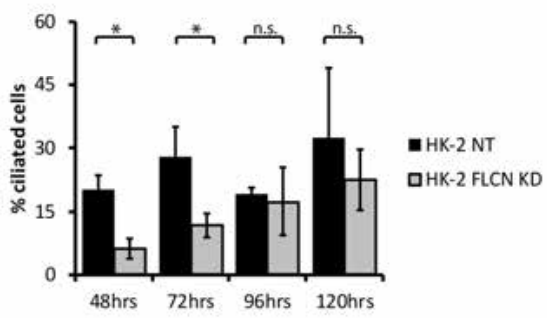

C

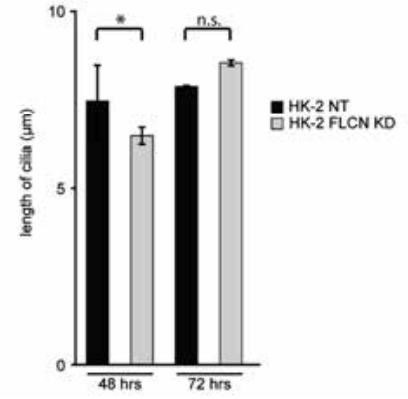

D

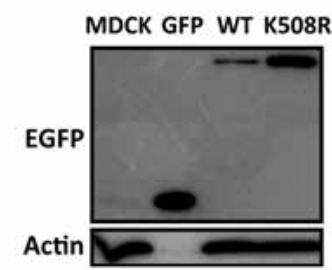

E

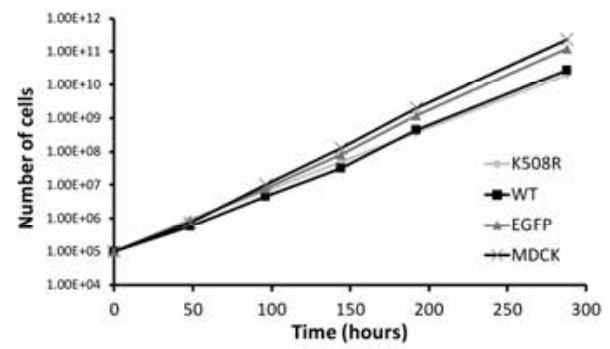

$\mathbf{F}$

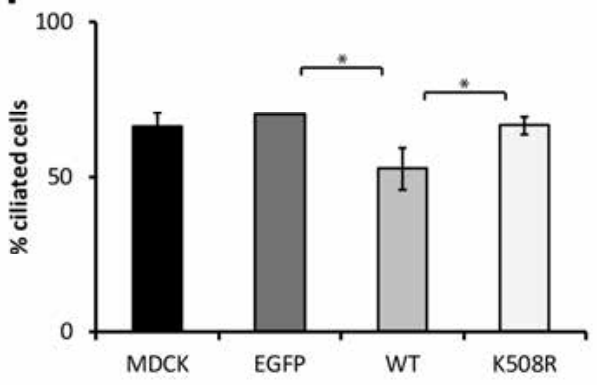

Figure 4. Knockdown and exogenous expression of FLCN affects ciliogenesis in kidney cells (continued)

(D) Western blot of FLCN expressing cell lines

Western blot probed with EGFP antibody showing MDCK cells with stable expression of EGFP alone, EGFP-FLCN WT and the disease-causing FLCN missense variant EGFP-FLCN K508R. Actin was used as a loading control. Actin is not detected in the EGFP only MDCK cells, as a result of 10 -fold dilution of this sample due to very high EGFP expression in this cell line

(E) Growth curve in FLCN expressing cell lines

At a starting density of $10^{5}$ cells per well, untransfected MDCK cells and MDCK cells with stable overexpression of EGFP, EGFP-FLCN WT or EGFP-FLCN K508R were plated. Cell number was determined at each time point. Each data point is the average of six independent counts. Error bars represent standard error of the mean.

(F) Measurement of ciliogenesis in FLCN expressing cell lines

Untransfected MDCK (MDCK) and MDCK cells stably overexpressing EGFP, EGFP-FLCN WT (WT) or EGFP-FLCN K508R (K508R) were maintained at confluence for 5 days, fixed and stained with acetylated alpha tubulin to mark the ciliary axoneme and with DAPI to stain nuclei. Cells expressing EGFP-FLCN WT showed a statistically significant reduction in the percentage of ciliated cells compared to cells expressing EGFP alone $(\mathrm{p}<0.001)$ or EGFP-FLCN K508R $(\mathrm{p}<0.001)$, as counted using ImageJ. Error bars represent standard error of the mean. * is $\mathrm{p}<0.05$ 
Exogenous expression of FLCN affects proliferation and ciliogenesis

Having noted that exogenous expression of FLCN in UOK257-2 cells seemed to reduce the number of cilia observed we then decided to examine the effect of exogenous FLCN expression on ciliation in more detail. To do this, we generated cell lines expressing EGFP alone or EGFP-FLCNWT from an expression construct. We also attempted to make stable cell lines expressing the different EGFP-tagged patient mutations described above. However, only the FLCN missense variant EGFP-FLCN K508R resulted in a usable stable cell line (Fig. 4D). We noted that the cell lines expressing exogenous EGFP-FLCN seem to grow more slowly than the cells expressing EGFP alone and we therefore measured the doubling time of these cells. As shown in Figure 4E and Supplementary Material, Table S2, expression of EGFP-FLCN WT and EGFP-FLCN K508R significantly increased the cellular doubling time, when compared with the control cell lines. This finding contrasts with previously published data, suggesting that a missense mutant (H255R) does not affect cell growth (8). Next, we tested the ability of these different cells to ciliate (Fig. 4F).

To initiate formation of cilia, all cells were cultured to 5 days post-confluence. Cells expressing EGFP-FLCN WT $(52.5 \% \pm 4.77 n=705)$ exhibited a statistically significant reduction in the number of cilia relative to the EGFP alone control (EGFP $70 \% \pm 0.12 n=825$ ). Interestingly, despite the apparently normal localization and similar growth defect to that observed for wild-type FLCN, EGFPFLCN K508R $(66.5 \% \pm 2.03, n=670)$ did not change the percentage of ciliated cells when compared with untransfected or cells expressing EGFP alone (Fig. 4F).

Supplementary Table 2. Average Doubling time of MDCK

\begin{tabular}{lcc}
\hline Cell Line & $\begin{array}{c}\text { Average doubling } \\
\text { time (hours) }\end{array}$ & $\begin{array}{c}\text { SEM } \\
\text { (hours) }\end{array}$ \\
\hline MDCK & 13.7 & 1.8 \\
MDCK EGFP & 14.3 & 1.2 \\
MDCK EGFP-FLCN & 17.3 & 1.1 \\
MDCK EGFP-FLCN K508R & 16.3 & 0.8 \\
\hline
\end{tabular}


Knockdown and exogenous expression of FLCN impairs kidney cell spheroid growth in three-dimensional culture

Having established that exogenous expression and knockdown of FLCN both adversely affect cilia number in monolayer culture, we then assessed the effect of modulating FLCN expression in a 3D culture system. Due to the complex signalling required for proper spatial orientation, a mild ciliopathy phenotype in monolayer culture can translate into a more severe one in 3D cultures (34). MDCK and IMCD3 cell lines cultured in 3D are both established models for renal morphogenesis, mimicking normal renal tubules by forming spheroids or tubules composed of polarized cells exhibiting a lumen lining a ciliated apical membrane. We considered that BHD cyst formation could be related to delayed ciliogenesis resulting from reduced levels of wild-type FLCN. Therefore, we assessed the effects of FLCN knockdown in detail in the 3D system. Spheroid formation in cells treated with siRNA against Flcn ( $\mathrm{SiFlcn}$ ) was severely impaired when compared with non-target siRNA (SiCON) (Fig. 5A i). The level of knockdown achieved was $~ 60 \%$ (Fig. 5A ii) and in line with previous data, fewer cilia were observed in Flcn knockdowns compared with non-target controls (Fig. 5A iii). The number of cells composing the median section of a spheroid did not differ significantly in the SiFlcn-treated cells versus control knockdown (Fig. 5B i). We then determined the overall and lumen size of these spheroids. As shown in Figure 5B ii, while SiFlcn only resulted in a small decrease in the overall size of the spheroid, it did cause a marked and significant decrease in luminal size when compared with the control spheroids. In addition to the effects of Flcn knockdown on cilia frequency and lumen size, we observed an increase in improper orientation of cell divisions. In normal spheroids, cell division occurs in plane with the apical membrane, directed towards the lateral membranes (Fig. 5C i). By scoring the number of cells that are either actively dividing in the wrong orientation, or have ended up outside the normal plane, we found that Flcn knockdown causes a strong increase in abnormally orientated cell divisions (Fig. 5C ii). Having observed that knockdown of Flcn affects spheroid formation in 3D cultures, we then assessed the effect of exogenous expression of EGFP-FLCN WT in 3D spheroids. Consistent with the previously observed growth defect in cells expressing EGFP-FLCN WT, the number of cells composing an EGFP-FLCN WT spheroid was reduced when compared with the control cell line (Supplementary Material, Fig. S7A). We then compared the overall and lumen size of the EGFP-FLCN WT spheroids to that of the EGFP alone control cell line (Fig. 5E i). Interestingly, spheroid formation in cell types expressing EGFP-FLCN WT was severely impaired, with smaller spheroids exhibiting a reduction (or absence) in the lumen size. Thus, both knockdown and exogenous expression of FLCN perturb lumen formation, with the latter giving a more severe phenotype. 


\section{BHD-associated FLCN missense mutation is partially dysfunctional with respect to spheroid formation}

Next, we tested the ability of the K508R mutant to influence spheroid formation, as this mutation does not seem to affect ciliogenesis. In contrast to EGFP-FLCN WT, EGFP-FLCN K508R spheroids appear similar to normal spheroids (Fig. 5D), where a clear lumen can be observed. The size of the spheroid and lumen was determined and while the overall size was similar, the lumen size was found to slightly deviate from EGFP controls, suggesting that K508R may have a mild effect on lumenogenesis. In line with the previous growth rate data, the number of cells composing an EGFP-FLCN K508R spheroid was reduced when compared with the control cell line (Supplementary Material, Fig. S7B).

These observations further suggest that the spheroid formation defect observed upon FLCN knockdown or exogenous expression could be linked to ciliary dysfunction.

Figure 5. Knockdown or expression of exogenous FLCN impairs kidney cell spheroid growth in threedimensional culture

(A) Knockdown of FLCN impairs kidney cell spheroid growth in three-dimensional culture (i) IMCD3 cells were treated with non-targeting RNAi (siCON) or FLCN RNAi (siFlcn) for 48 hours. The cells were subsequently split into 2 equal parts, with the first half cultured for three days in matrigel and the remainder in 2D culture for quantification of knockdown shown in (ii). Cultures were fixed and stained. Tight-junction marker Z0-1 (orange) localises to the apical side, $\beta$-catenin (green) is enriched at lateral cell-cell contacts and acetylated alpha tubulin stains cilia (red) that are present protruding into the lumen. Nuclei are stained with DAPI. Representative spheroids are shown. SiCON cells form a typical spheroid that is evenly rounded and has a clear lumen. Cells treated with SiFlcn form spheroids with impaired ciliogenesis, reduced overall and luminal sizes. Arrows indicate cilia containing $\beta$-catenin. Scale bar is $10 \mu \mathrm{m}$. (ii) Quantification of Flcn levels using RT-PCR in IMCD3 cells treated with siCON or siFlcn (normalised to Rpl19). The RNA was extracted from cells at the same time point as the 3D spheroids shown in (i) were fixed. (iii) Quantification of the number of cilia observed in non-target siCON or the siFlcn spheroids. Spheroids with reduced Flcn levels exhibit significantly decreased numbers of cilia compared to the siCON control ( $p>0.0001)$. (B) Knockdown of FLCN in IMCD3 decreases spheroid and lumen size in three-dimensional culture IMCD3 cells with siRNA mediated knockdown of Flcn form spheroids that consist of the same number of cells (i) but are significantly smaller (ii) in size $(\mathrm{p}=0.0058)$ and have a smaller lumen (ii, $\mathrm{p}=0.0003$ ) compared to control spheroids. (C) Knockdown of FLCN impairs spindle orientation in three-dimensional culture (i) Representative image of misoriented, dividing cell and a cell that ended up outside the normal plane (white arrows) in siFLCN spheroid versus control. Cells are stained with DAPI. Scale bar $20 \mu \mathrm{m}$. (ii) Quantification of cell division orientation inIMCD3 spheroids treated with SiFlcn, which show a significant increase in the number of misorientated spindles per spheroid compared to control spheroids $(\mathrm{p}<0.0001)$. (D) Expression of wild type but not K508R FLCN impairs kidney cell spheroid growth in three-dimensional culture MDCK cells stably overexpressing EGFP, EGFP-FLCN WT or EGFP-FLCN K508R were grown in collagen for 11 days. Cultures were fixed and stained with DAPI (blue). Note the lack of lumen formation in the cells overexpressing EGFP-FLCN WT in the 2D and 3D projection. Scale bar is $20 \mu \mathrm{m}$. (E) Quantification of spheroid parameters after FLCN expression in IMCD3 (i) IMCD3 cells with stable expression of EGFP-FLCN WT form spheroids that are significantly smaller in size $(p<0.0001)$ and have a smaller lumen $(p<0.0001)$ compared to control spheroids expressing EGFP only. (ii) Expression of EGFP-FLCN K508R results in normal-sized spheroids $(\mathrm{p}=0.2381)$ with a slight reduction in lumen size $(\mathrm{p}<0.0001)$ compared to control spheroids expressing EGFP only. 

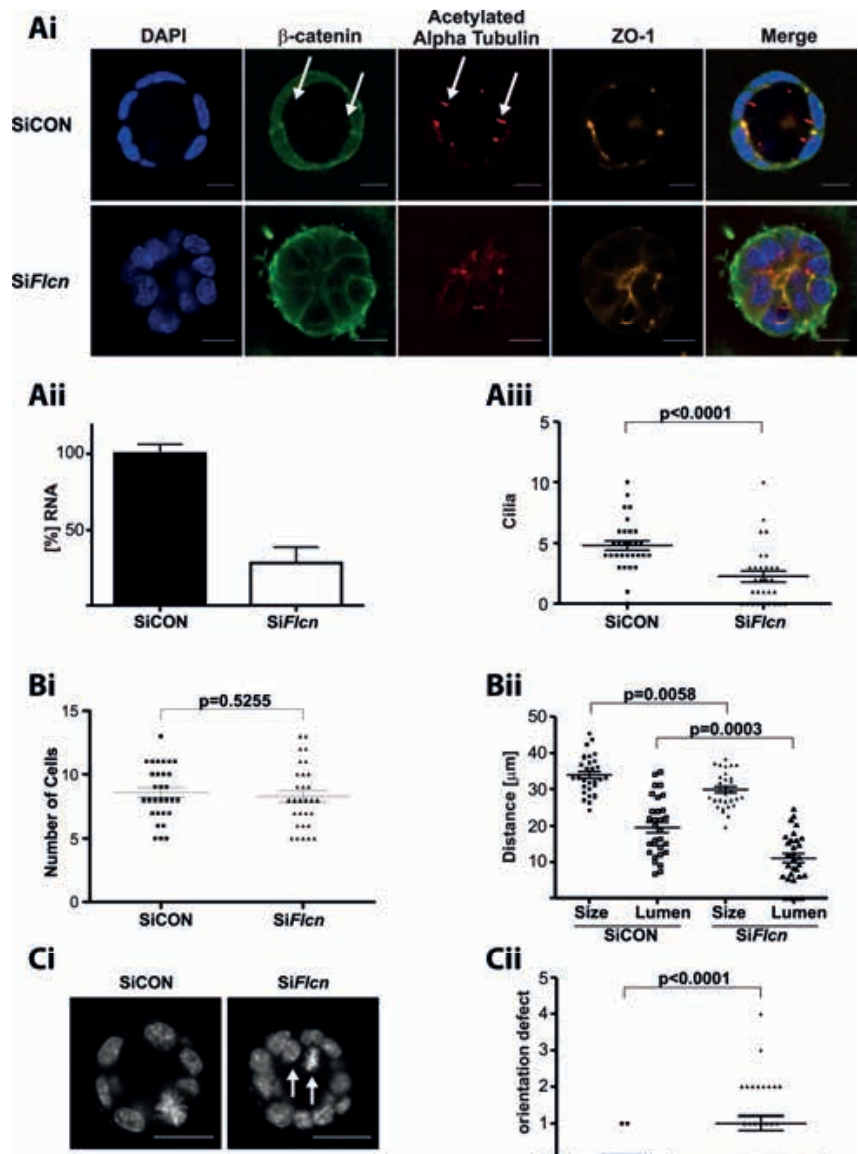

Cii

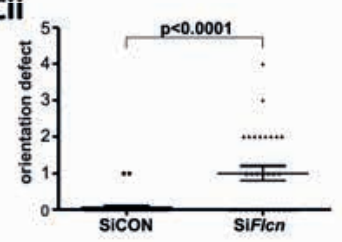

D

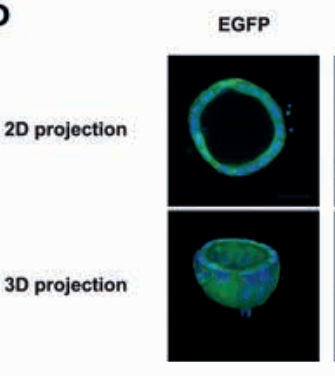

Ei
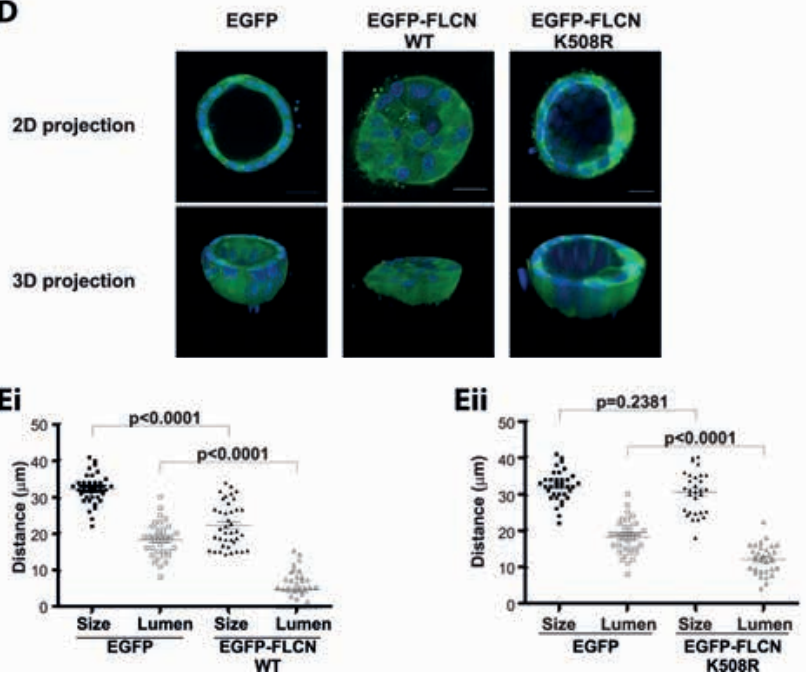

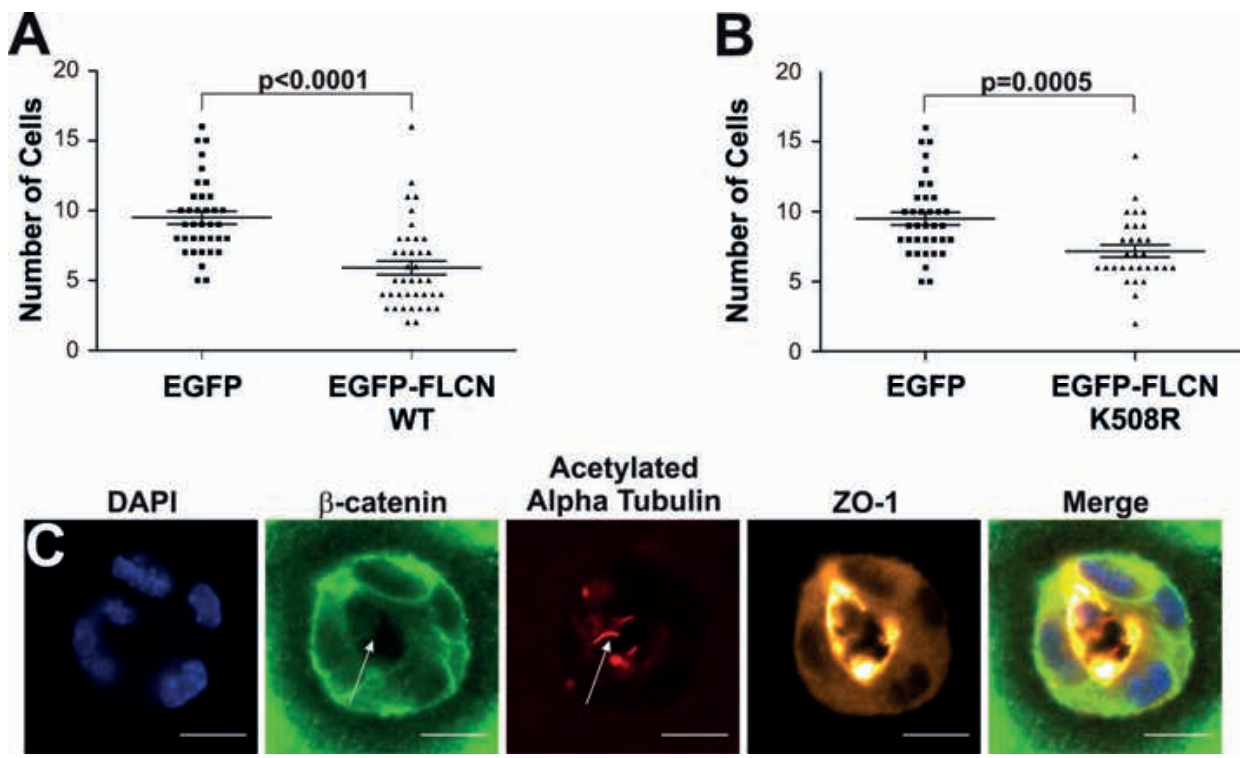

Acetylated Alpha Tubulin
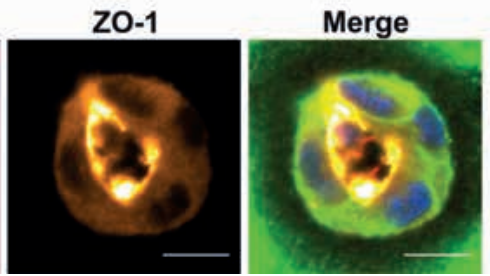

Supplementary figure S7. Spheroid formation in IMCD3 cells

(A) Quantification of EGFP-FLCN WT spheroids versus the control reveals a significant reduction in the number of cells composing a spheroid $(\mathrm{p}<0.0001)$.

(B) Quantification of EGFP-FLCN K508R spheroids versus the control reveals a significant reduction in the number of cells composing a spheroid $(\mathrm{p}=0.0005)$.

(C) Lack of localisation of $\beta$-catenin to cilia in siFlcn spheroids.

IMCD3 cells were treated with FLCN RNAi (siFlcn) and cultured for three days in matrigel. Cultures were fixed and stained. Tight-junction marker ZO-1 (orange) localises to the apical side, $\beta$-catenin (green) is enriched at lateral cell-cell contacts and acetylated alpha tubulin stains cilia (red) that are present protruding into the lumen. Nuclei are stained with DAPI. Arrows indicate position of cilia. To rule out the possibility of low level of $\beta$-catenin in cilia a long exposure time was used. Scale bar is $10 \mu \mathrm{m}$. 
Flcn knockdown results in $\beta$-catenin delocalization and activates canonical Wnt signalling

In the spheroid assays, we used $\beta$-catenin as a standard marker for the basolateral membranes. When we performed these assays, we noted that $\beta$-catenin can often be observed in the cilium (Fig. 5Ai, indicated by arrow). Intriguingly, in the few cilia present in the spheroids treated with SiFlcn, we rarely observed this localization of $\beta$-catenin (Supplementary Material, Fig. S7C). The sequestration of $\beta$-catenin to cilia has been reported to dampen canonical Wingless signalling (44) and abnormal Wnt/ $\beta$-catenin signalling has previously been implicated in renal cystogenesis (45). Therefore, we hypothesized that the observed defects in spheroid formation upon knockdown of Flcn might be a consequence of altered Wnt signalling. Thus, we examined canonical Wnt activity by looking at $\beta$-catenin phosphorylation and Axin2/CyclinD1 mRNA levels. Upon SiFlcn treatment, no reduction in the total amount of $\beta$-catenin was evident when compared with control RNAi (Fig. 6A). However, an antibody against the active, unphosphorylated form of $\beta$-catenin showed clear activation of $\beta$-catenin upon Flcn knockdown. Furthermore, quantitative RT-PCR of downstream target genes Axin2 and CyclinD1 shows up-regulation of these two genes upon Flcn knockdown, further supporting activation of the canonical Wnt pathway (Fig. 6B).

A

Anti- $\beta$-Catenin (active)
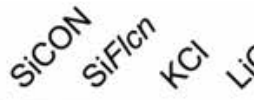

Anti- $\beta$-Catenin (total)

Anti- $\beta$-Tubulin

B

Axin2

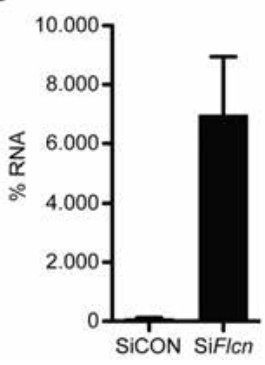

CyclinD1

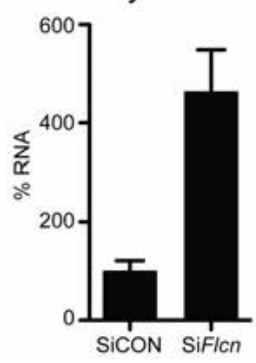

Figure 6. FLCN knockdown causes activation of canonical WNT signalling

(A) Knockdown of FLCN results in activation of $\beta$ catenin. Western blot on $30 \mu \mathrm{g}$ of whole cell lysate from treated IMCD3 cells in 2D culture. Cells were harvested at 60 hours post transfection. Cells were serum starved for $18 \mathrm{hrs}$ prior to harvesting. IMCD3 express equal amounts of total $\beta$-catenin (middle panel) upon treatment with non-targeting SiCON or SiFlcn or treatment with $20 \mathrm{mM} \mathrm{KCl}$ or $\mathrm{LiCl}$ for 16 hours. Using an antibody to detect the unphosphorylated, active $\beta$-catenin, cells treated with $\mathrm{LiCl}$ (an activator of $\beta$-catenin through inhibition of GSK3 $\beta$ ) showed increased active $\beta$ catenin as did SiFlcn treated cells (upper panel). The $\beta$-actin (lower panel) was used as a loading control.

(B) Activation of downstream targets of the WNT pathway upon FLCN knockdown. The activation of the WNT pathway was further confirmed using quantitative RT-PCR of downstream target genes Axin2 and CyclinD1. Upon Flcn knockdown, mRNA levels of both genes exhibited upregulation. 


\section{Discussion}

BHD patients show cyst formation in lungs, kidney and liver (6). Cyst formation in multiple organ systems is a hallmark characteristic of ciliopathies (23), which led us to hypothesize that ciliary dysfunction could contribute to the BHD phenotype. Our results suggest that this is indeed the case and provide a possible explanation for key aspects of the BHD phenotype. In a kidney sample taken from a BHD patient during surgery for renal cancer, we observed a cyst and a tumor in close proximity. Interestingly, we found similar arrangements in Nihon rat kidneys (data not shown). It is well established that in this model, cancer is preceded by the appearance of cuboidal cells with a clear cytoplasm lining dilated collecting ducts, followed by cells with acidophilic cytoplasm (33). The cancers that appear around 8 weeks consist of both clear and acidophilic cells. Large cysts such as the one shown in Figure 1E and $\mathrm{G}$ have not been reported as a microscopic finding, but macroscopic ones have (33). Of note, the cysts that we observed in the rat are lined with clear cuboidal cells; acidophilic cells grow into the cyst lumen (Fig. 1G). The tumors contain both cell types, with the clear cells consistently located towards the periphery. We note that human BHD RCC show the same arrangement of cells (3). We suggest from these observations that in humans with BHD, as in the Nihon rat, tumors develop from cysts. Hence, understanding cyst formation can help elucidate carcinogenesis in BHD.

\section{FLCN is a dose-dependent regulator of cilia formation}

Ciliogenesis is a process requiring complex regulation that is not yet fully understood. Entrance into the G0 phase of the cell cycle and establishment of cell polarity are considered to be the first prerequisites for ciliation (46). We observe that levels of FLCN affect the timing of ciliation. We also show that endogenously expressed FLCN, and tagged versions of FLCN, very specifically localize to tubulinbased structures such as motile and non-motile cilia, centrosomes and the mitotic spindle in a variety of cell types. HK-2 cells with reduced endogenous FLCN expression show reduced ciliation and a subtle but significant decrease in ciliary length at early starvation time points, which are subsequently overcome. These findings indicate that cells with less FLCN have a delay in ciliogenesis, compared with normal cells. IMCD3 spheroids recapitulate this reduction in ciliation upon knockdown of Flcn. Furthermore, the expression of exogenous wild-type FLCN also resulted in reduced ciliation, suggesting that tight regulation of FLCN levels is required for normal ciliogenesis.

We have not observed any obvious defects in the size or shape of matured cilia, indicating that once ciliogenesis has been initiated, FLCN's role is permissive. In line with other renal cell types examined (MDCK and HK-2), under conditions of confluence, UOK257 cells do have the ability to form cilia. However, despite the apparently normal appearance of these cilia in UOK257 cells, the actual percentage of cells exhibiting cilia is low relative to other cell lines examined. Similarly, 
ciliation is delayed in these UOK257 cells. The low level of and delay in ciliation observed may be a consequence of a reduced level of full-length FLCN expression in these cells. The abnormal ciliation in UOK257-2 is consistent with our observations in other cell types and suggests that FLCN expression levels are critically important for correct timing of ciliogenesis.

\section{FLCN regulates canonical Wht signalling and spindle orientation in spheroids}

We previously described the IMCD3 spheroid culture system as a suitable method to assess ciliopathy genes and their effects on renal morphology (34). The siRNAmediated knockdown of FLCN in IMCD3 cells demonstrates a role for the protein in establishing cellular orientation, as the spheroids formed by these knockdown cells exhibit an increased number of cells outside of the normal plane of division. This mitotic spindle misalignment may reflect disturbed PCP (controlled by noncanonical Wnt signalling), abnormal cortical microtubule function or mislocalized polarity proteins. Cilia have a role in the process of polarization by dampening the activity of the canonical Wnt pathway via sequestration of $\beta$-catenin (44). Accordingly, FLCN knockdown seems to reduce ciliary $\beta$-catenin retention and is associated with an increase in the level of unphosphorylated $\beta$-catenin. The lack of phosphorylation of $\beta$-catenin results in the stabilization of the protein and an increase in canonical Wht activity (47). As a consequence, non-canonical PCP might be inhibited (48), resulting in spindle misalignment. In view of the increased Wnt activity, it is of considerable interest that recent observations clearly show an increased incidence of kidney cancer in people who chronically use lithium chloride for psychiatric conditions (49). Lithium activates canonical Wnt signalling through repression of GSK3 $\beta$ activity (50). The spectrum of renal tumors associated with lithium use is remarkably similar to that seen in BHD. Thus, we propose that carcinogenesis in BHD may be partly due to deregulated canonical Wnt signalling.

An alternative explanation for the spindle orientation defect could be that FLCN does not exert a direct effect on PCP signalling, but rather is actively involved in spindle orientation. The observation that EGFP-FLCN WT localizes to the centrosome and mitotic spindle is compatible with this idea. Other ciliary proteins have been shown to affect spindle positioning. For instance, the classic ciliary transport protein IFT88 localizes to the centrosome in mitosis, where it plays a fundamental role in microtubule transport (39). FLCN might exert a similar function in controlling essential processes at the spindle pole, defects of which result in misorientation of the mitotic spindle. Finally, a recent report describes that LKB1 regulates spindle orientation through AMPK, possibly through microtubule destabilization (40), and we have previously demonstrated an increase in AMPK phosphorylation in UOK257 cells (14).

Very recently, Nookala et al. (51) provided evidence that the C-terminal half of FLCN may act in vitro as a guanine nucleotide exchange factor for Rab35, a poorly characterized GTPase involved in endocytotic membrane recycling and in the final 


\section{CHAPTER 2}

steps of cytokinesis (52). How this function relates to the pervasive cellular abnormalities caused by FLCN's absence and the BHD phenotype is not yet clear. However, both ciliogenesis and mitotic spindle positioning require vesicular transport, which exhibit significant overlap (53) and we show that these processes are affected in BHD. Thus, FLCN may have a function in intracellular transport events involved in ciliogenesis.

\section{FLCN might exhibit a partial genotype-phenotype correlation}

To date, no clear correlation has been found between mutations in FLCN and the severity of BHD symptoms observed in patients. However, our findings with FLCN K508R may indicate that there might be a milder form of BHD associated with specific missense mutations. The growth defect caused by K508R and its cellular localization are indistinguishable from the wild-type protein. However, in contrast to wild-type FLCN, expression of K508R does not seem to adversely affect ciliogenesis and only mildly affects the spheroid forming abilities of the cell. These results are suggestive of at least some (partial) functionality of K508R mutant. This is in line with the published clinical information (54). The patient, in whom the mutation was found, presented with bilateral multifocal benign renal oncocytomas and was only diagnosed with BHD upon sequencing of the oncocytomas. At the time of publication, they did not manifest any of the other known BHD symptoms (W.M. Linehan, personal communication, 2013). It is tempting to speculate that, if kidney cancer in BHD arises from cysts, mutations that do not affect ciliogenesis will not be associated with malignancy. Another missense mutation (p.Arg239Cys) has been reported in a patient presenting with kidney cancer (55). If our reasoning is correct, this particular mutant should affect ciliogenesis.

\section{Mutant FLCN alleles might contribute to the phenotype}

From our observations, we think that the clinical manifestations of BHD may not result from simple loss of one FLCN allele. Rather, the mutant versions of FLCN could also contribute to the BHD phenotype, perhaps in a dominant negative manner. Relative to the UOK257 cell lines, the restoration and exogenous expression of FLCN in UOK257-2 cells does not increase the low percentage of ciliated cells upon confluence. Mutant versions of FLCN that were tested localized to the centrosome and (at least for K508R) negatively impact cellular proliferation. Expression of truncated or wild-type FLCN seems to impede ciliogenesis (with the notable exception of K508R).

Complete loss of FLCN has been shown to be lethal $(9,13)$ and we would therefore not expect to encounter it in patients or in any tissue. Accordingly, the majority of BHD-associated kidney tumors have somatic second hits affecting FLCN, rather than loss of the wild-type allele (56). We also show that truncated FLCN is still expressed in a renal cancer from a BHD patient (Fig. 1 and (32)). Finally, people with the Smith-Magenis syndrome (MIM182290), a congenital disorder caused by heterozygous deletion of chromosome 17p11.2 (including the 
region containing the $F L C N$ locus), do not develop symptoms consistent with BHD (57). In particular, patients do not exhibit an elevated risk of developing kidney cancer.

Based on these observations, we propose that a 'just right' hypothesis, as for the APC tumor suppressor (58), also holds true for FLCN.

In conclusion, we provide evidence that BHD syndrome is a novel ciliopathy. The causative protein, FLCN, localizes to motile and non-motile cilia, centrosomes and the mitotic spindle. It is involved in the initiation of ciliogenesis-once ciliation is underway, FLCN's role seems to be permissive. Knockdown of FLCN affects PCP, which might be due to the ciliation defect. Additional roles for FLCN in establishing cellular polarity seem likely and we have evidence for abnormal canonical Wnt signalling in the context of FLCN knockdown. Wnt signalling is potentially oncogenic and might thus present a new mechanism for carcinogenesis in BHD. Our data further suggest that the correct level of FLCN expression is critically important for its optimal function. Delayed ciliogenesis is likely to affect the ability of cells to orientate themselves in newly generated polarized tissues. The observations in Nihon rat kidney suggest that cyst formation may be a prerequisite to cancer development. Hence, FLCN mutations that do not affect ciliogenesis, such as K508R, might not cause cancer and it would be of considerable interest to test this hypothesis using knock-in mouse models.

The classification of BHD as a ciliopathy should help better understand its pathogenesis, as the study of BHD can now benefit from the intense research effort on ciliopathies. 


\section{Materials and methods}

DNA constructs and antibodies

Human FLCN was cloned into pEGFP C1 (Clontech, Mountain View, CA, USA) using EcoRI. EGFP-tagged FLCN mutant constructs were made by site-directed mutagenesis using the Quikchange II Site Directed Mutagenesis kit according to the manufacturer's instructions (Agilent, Santa Clara, CA, USA) with the following primers (Supplementary Material, Table S3). EGFP-tagged FLCN WT and mutants were cloned into the pQCXIP vector (Clontech) using AgeI and EcoRI.

Human-specific FLCN shRNA plasmid pLKO.1-puroshRNA5968 and the Non Target shRNA plasmid pLKO.1- puro-NonTaget (Sigma) were a kind gift from Prof. A. Pause (McGill University, Canada). Murine-specific Flcn siRNA oligos were purchased from Dharmacon (Lafayette, CO, USA).

Primary antibodies used for immunofluorescence microscopy are anti-FLCN-AP (rabbit polyclonal, a kind gift from Prof. A. Pause, 1:200), anti-FLCN (rabbit monoclonal, 3697 Cell Signalling, Danvers, MA, USA 1:200), anti-FLCN [rabbit polyclonal, (12) a kind gift from Dr T. Cash 1:100], anti-pericentrin (rabbit polyclonal, ab4448 Abcam, Cambridge, UK, 1:1000), anti-gamma-tubulin (mouse monoclonal, T3559 Sigma 1:1000), anti-acetylated-alpha-tubulin (mouse monoclonal, ab24610 Abcam 1:200), anti-acetylated-alpha-tubulin (mouse, T7451 Sigma, 1:20 000), anti- $\beta$-catenin (rabbit, AH00462 Invitrogen, Paisley, UK, 1:500) and anti-ZO-1 (rat, R40.76 Santa Cruz Biotechnology, Santa Cruz, CA, USA, 1:500).

Secondary antibodies used were goat-anti-mouse Texas red (1010-07 Southern Biotech, Birmingham, AL, USA, 1:80), goat-anti-rabbit FITC (4050-02 Southern Biotech, 1:80), goat-anti-mouse Cy5 (1034- 15, Southern Biotech, 1:80) and antiRabbit-488, anti-mouse-568 and anti-Rat-647 (all from Molecular Probes, Invitrogen).

Supplementary Table 3. Primer sequences used for site-directed mutagenesis

\begin{tabular}{ll}
\hline Construct & Primer Sequence \\
\hline pEGFP-FLCN H255R & GGCAAAGGAGGTGCGCAGGCACGC \\
pEGFP-FLCN H255R & GCGTGCCTGCGCACCTCCTTTGCC \\
pEGFP-FLCN H429 & AGGAGAGCACGTGGGGGGGGGATCT \\
pEGFP-FLCN H429 & AGATCCCCCCCCACGTGCTCTCCT \\
pEGFP-FLCN Y463X & GGTCACCACAAACTCCTACTTGCTGAGAGACTG \\
pEGFP-FLCN Y463X & CAGTCTCTCAGCAAGTAGGAGTTTGTGGTGACC \\
pEGFP-FLCN K508R & CCACTCCTCCCTGAGGCAGACGAG \\
pEGFP-FLCN K508R & CTCGTCTGCCTCAGGGAGGAGTGG \\
\hline
\end{tabular}


Primary antibodies used for western blot were raised against EGFP (mouse, 11814460001 Roche, Basel, Switzerland 1:2000), actin (mouse monoclonal, A5441 Sigma 1:5000), beta-tubulin (ab6046, Abcam, 1:500), gamma-tubulin (mouse monoclonal, T3559 Sigma 1:1000), total $\beta$-catenin (Ab6302, Abcam, 1:400) and unphosphorylated $\beta$-catenin (ABC; clone 8E7, Millipore, 1:400).

Secondary antibodies used include HRP-conjugated goat-anti-mouse and goatanti-rabbit (Jackson Immuno Research Laboratories, West Grove, PA, USA, 1:10 000).

Custom-made rabbit antibodies against the $\mathrm{N}$-terminus and C-terminus of FLCN were generated and affinity purified by Eurogentec (Seraing, Belgium). The Nterminal antibody was raised against a peptide consisting of amino acids 29-41 (LPQGDGNEDSPGQCE) and the C-terminal antibody was raised against a peptide consisting of amino acids 522-536 (TKVD SRPKEDTQKLL) of human FLCN (NP_659434).

\section{Cell lines and cell culture}

Normal Human Dermal Fibroblasts (NHDF) were purchased from Lonza (Blackley, UK). MDCK cells type II were a gift from P. Zimmermann (Department of Human Genetics, University of Leuven, Belgium). HK-2 cells were provided by Prof. Dr. M. van Engeland (Department of Pathology, Maastricht University Medical Centre). UOK257 and UOK257-2 cell lines were kindly supplied by Dr L. Schmidt (NIH, USA). All cells were cultured in tissue culture grade plastics, in Dulbecco's modified Eagle medium (DMEM) containing $4.5 \mathrm{~g} / \mathrm{l} \mathrm{D}$-glucose and L-glutamine (Lonza) supplemented with $10 \% \mathrm{FCS}$ (Lonza) and $50 \mu \mathrm{g} / \mathrm{ml}$ gentamycin (Lonza) at $37^{\circ} \mathrm{C}$ in $5 \%$ CO2 (humidified atmosphere). The culture medium was changed every 2 to 3 days. At confluence, cells were split using trypsin-EDTA (Lonza). Primary ciliated HNE cells were obtained from consenting donors with no known history of BHD under local ethical approval from East of Scotland Research Ethics Service (EoSRES) REC1 (12/ES/ 0081). A 2 mm diameter cytology brush was inserted into each nostril and cellular material was recovered by abrasion of the nasal turbinate. This was washed with several changes of serum free Medium 199 containing $1 \mathrm{mM}$ Dithiothreitol (Sigma) and Primocin antibiotic/antimycotic (Invivogen, Toulouse, France). Cells were either processed immediately for immunofluorescence or maintained in culture for up to 1 week by placing into Bronchial Epithelial Growth Medium (Lonza), supplemented with Single- Quot Kit Supplements and appropriate growth factors. Human sperm was obtained from volunteer donors (healthy men with no known fertility problems randomly selected from the general public) who were recruited in accordance with the HFEA Code of Practice (version 8) under ethical approval (08/S1402/6) from the EoSRES REC1. Capacitated sperm cells were prepared using a swim-up assay (59) and were then immediately processed for immunofluorescence by spreading $10 \mathrm{ml}$ sperm solution onto Polysine slides (VWR, Leicestershire, UK). Slides were air-dried prior 


\section{CHAPTER 2}

to fixation in Methanol:Acetone (1vol:1vol) for $20 \mathrm{~min}$ at $-20^{\circ} \mathrm{C}$ and staining was performed as described for 2D cultures.

\section{Immunocytochemistry of $2 D$ cultures}

For fluorescent microscopy, cells were cultured on glass cover slips. Cilium formation was induced by culturing the cells for at least 4 days after reaching confluence or by serum starving the cells for at least $48 \mathrm{~h}$ in DMEM without FCS, depending upon the cell type. When ready for staining, cells were fixed using either ice-cold $100 \%$ methanol or $4 \%$ formaldehyde at room temperature and permeabilized using $0.2 \%$ Triton $\mathrm{X}-100$, depending upon the primary antibody. Cells were incubated overnight at $48 \mathrm{C}$ in primary antibody appropriately diluted in $3 \%$ BSA in TBS block buffer followed by $1 \mathrm{~h}$ incubation at room temperature in secondary antibody appropriately diluted in block buffer. DNA was stained with DAPI. Fluorescent samples were visualized using a Leica TCS SPE confocal laser scanning fluorescence microscope (Leica DMRBE, Mannheim, Germany). Images were further processed using Image $(\mathrm{NIH})$ software to determine the percentage of ciliated cells ( $n=3$, average 200 cells per group per experiment) and cilia length ( $n=3$, average 40 cilia per group per experiment).

\section{Immunohistochemistry}

Sections of $4 \mu \mathrm{m}$ were processed using standard methodology; antigen retrieval was performed after blocking in $\mathrm{H}_{2} \mathrm{O}_{2}$, using $10 \mathrm{mM}$ citrate buffer $\mathrm{pH}$ 6.0. For FLCN stain of BHD kidney sections, the custom-made C-terminal, N-terminal antibodies, or FLCN AP antibody was diluted in 3\% BSA in PBS. To visualize we used the Dako REAL EnVision Detection System Peroxidase/ DAB + Rabbit (Dako Heverlee, Belgium). The slides were counterstained with haematoxylin and embedded in Entellan.

\section{Transfection and generation of stable cell lines}

Transient transfections were performed using FuGENE HD Transfection Reagent (Roche) according to the manufacturer's instructions. To generate EGFP expressing stable cell lines, cells were transfected with appropriate plasmid DNA using FuGENE HD. Twenty-four hours post transfection cells were selected using 3 $\mu \mathrm{g} / \mathrm{ml}$ puromycin (Invivogen). Clonal cell populations were picked using cloning cylinders and analysed for the expression of the protein of interest by western blot. To generate FLCN knockdown and non-target cell lines, HK-2 cells were transfected with appropriate plasmid DNA using FuGENE HD. Twenty-four hours after transfection selection of $1 \mu \mathrm{g} / \mathrm{ml}$ puromycin (Invivogen) was added. Clonal cell populations were picked using cloning cylinders and subsequently analysed by western blot for FLCN expression. 


\section{Growth curve}

MDCK cells were seeded into six-well plates at a starting density of $10^{5}$ cells per well. The cell number was determined at $48 \mathrm{~h}$ intervals using a haemocytometer. After counting cells were reseeded into a fresh six-well plate at an appropriate sub-confluent density and allowed to grow for another $48 \mathrm{~h}$.

\section{$3 D$ spheroid growth}

MDCK cells were resuspended in a collagen matrix and seeded on a glass cover slip. The mixture was allowed to polymerize for $30 \mathrm{~min}$ at $37^{\circ} \mathrm{C}$, and fresh medium was put on top to cover the matrix. After 11 days spheroids had formed. IMCD3 cells were cultured and reverse transfected with $50 \mathrm{nM}$ On-TargetPlus siRNA murine-specific oligos (Dharmacon) using Lipofectamine RNAimax (Invitrogen) according to the manufacturer's recommendations. Twenty-four hours later, cells were collected and resuspended, mixed 1:1 with growth factor-depleted Matrigel (BD Bioscience, San Jose, CA, USA) and seeded in a glass bottom Lab-tek II chamber (Nunc Thermo Scientific). The mixture was allowed to polymerize for $30 \mathrm{~min}$ at $37^{\circ} \mathrm{C}$, and fresh medium was put on top to cover the matrix. After 3 days spheroids had formed.

\section{Immunocytochemistry of $3 D$ cultures}

After spheroid formation, the medium was removed and the gels containing the spheroids were washed with warm PBS supplemented with calcium and magnesium and fixed in fresh $4 \%$ PFA for $30 \mathrm{~min}$ at room temperature. After washing with PBS, the cells were permeabilized for $20 \mathrm{~min}$ in gelatin dissolved in warm PBS (7 mg/ml) with 0.5\% Triton X-100 added. Primary antibodies were diluted in the permeabilization buffer and the gels were incubated at $4^{0} \mathrm{C}$ overnight. After washing the spheroids three times for $30 \mathrm{~min}$ in permeabilization buffer, secondary antibodies were diluted in permeabilization buffer and the gels were incubated overnight at $4^{0} \mathrm{C}$. The next day, the gels were washed three times in permeabilization buffer immersed in Fluoromount-G (Cell Lab, Beckman Coulter, Brea, CA, USA). Images were taken with a Zeiss LSM510 inverted confocal microscope. Using confocal laser scanning microscopy, several parameters of these spheroids can be determined, including the number of cells forming the median section of a spheroid. Additionally, the total spheroid size (basal membrane) and the luminal size (apical membrane) can be determined by measuring the distance of three lines spanning the maximal distance at a roughly $120^{\circ}$ angle of one another. Also the number of cilia present parallel to the transverse section (over a 6-8 $\mu \mathrm{m}$ Z-section depending on spheroid size) can be counted.

\section{Statistical analyses}

The percentage of ciliated cells ( $n=3$, average 200 cells per group per experiment) was analysed using a two-tailed Pearson's Chi-square test for categorical data (significant $\mathrm{P}<0.05$ ). Cilium length (number of experiments $=3$, average 40 cilia 


\section{CHAPTER 2}

per group per experiment) and all spheroid parameters were analysed using nonparametric two-tailed Mann-Whitney tests (significant $\mathrm{P}=0.05$ ). For SiCON, SiFlcn and EGFP-FLCN K508R cells 30 spheroids were analysed, for EGFP cells 36 spheroids were analysed and for EGFP-FLCN WT 43 spheroids were analysed. Distances are averages of three measurements at a $120^{\circ}$ angle of each other in $\mu \mathrm{m}$.

\section{RNA isolation and quantitative RT-PCR}

RNA was isolated using the RNA isolation kit from Qiagen (Hilden, Germany), according to the manufacturer's recommendations. Probes Flcn mm00840973_m1 and Rpl19 Mm01606 037_g1 form RNA analysis were purchased from Applied Biosystems. Real-time PCR was performed in triplicate using the one-step RT-PCR kit and the 7500 system from Applied Biosystems. Normalized Ct values were used to calculate relative expression levels per condition and to determine the standard deviation ( $\mathrm{P}=0.05$; $\mathrm{n}=3$ ). Real-time PCR for Axin2 and CyclinD1 was performed as previously described (60) using the primers listed in Supplementary Material, Table S4.

Supplementary Table 4. Primer sequences used for real-time PCR of Axin2 and CyclinD1

Cyclin D1 gtgctgcgaagtggaacc

Cyclin D1 atccaggtggcgacgatct

Axin2 agccaaagcgatctacaaaagg

Axin2 ggtaggcattttcctccatcac

\section{Sequencing}

$1 \times 10^{6}$ UOK257 and HK-2 cells were harvested using trypsin- EDTA. Genomic DNA was isolated using the Genomic DNA purification kit from Gentra Systems (Minneapolis, MN, USA) according to the manufacturer's recommendations. Subsequently, the BHD locus was sequenced using primers directed to intronic sequences adjacent to the FLCN exons.

\section{Western blotting}

Cells were harvested using typsin-EDTA and whole cell lysate (WCL) was obtained using NP-40 lysis buffer (150 mM NaCl, 1\% NP-40, $250 \mathrm{mM}$ Tris pH 7.3) supplemented with protease and phosphatase inhibitors (Roche). Protein concentration was determined using the Bradford protein assay (Sigma). An appropriate volume of Laemmli sample buffer was added to equal amounts of total protein followed by boiling for $5 \mathrm{~min}$. Samples were subjected to SDS-PAGE electrophoresis before transfer to an Amersham Hybond ${ }^{\mathrm{TM}}$-PVDF membrane (GE Healthcare Life Sciences, Buckinghamshire, UK). After overnight incubation at $4^{0} \mathrm{C}$ in primary antibody appropriately diluted in $0.5 \%$ BSA in TBS block buffer, membranes were incubated with secondary antibody appropriately diluted in 
block buffer. Signal was detected using enhanced chemiluminescence (ECL) system (Thermo Scientific, Waltham, MA, USA).

\section{Supplementary material}

Supplementary Material is available at $H M G$ online.

\section{Acknowledgements}

We thank Prof. P.J. van Diest for pathological analysis of immunohistochemistry and F. Meeuwsen for technical support. The authors are grateful to Dr L. Schmidt (NIH) for kindly providing the UOK257 and UOK257-2 cell lines. We thank Prof. A. Pause for FLCN AP antibody, Dr T. Cash for FLCN antibody. MDCK type II cells were a kind gift from P. Zimmermann. HK-2 cells were a kind gift from Prof. M. van Engeland. Conflict of Interest statement. None declared.

\section{Funding}

This work was supported by Dutch Cancer Society (grant KWF U2009-4352), Association for International Cancer Research (grant AICR 11-0687), Annadal Foundation and GROW (M.V.S. ,B.J.C, M.L., T.C. and M.V.), the European Community's Seventh Framework Programme FP7/2009 agreement no: 241955, SYSCILIA and the Netherlands Organization for Scientific grant NWO Vidi917.66.354 (R.H.G.), Myrovlytis Trust (R.P.H), the Wellcome Trust (grant 088032/z/08/z) (S.L.), Myrovlytis Trust (R.N.); Association of International Cancer Research Career Development Fellowship (06-914/ 915), AICR (grant 11-0687) and Myrovlytis Trust (A.T.). 


\section{CHAPTER 2}

\section{References}

1. Hornstein OP, Knickenberg M. Perifollicular fibromatosis cutis with polyps of the colon--a cutaneo-intestinal syndrome sui generis. Archives for dermatological research Archiv fur dermatologische Forschung. 1975 Sep 12;253(2):161-75. PubMed PMID: 1200700.

2. Birt AR, Hogg GR, Dube WJ. Hereditary multiple fibrofolliculomas with trichodiscomas and acrochordons. Archives of dermatology. 1977 Dec;113(12):1674-7. PubMed PMID: 596896.

3. Houweling AC, Gijezen LM, Jonker MA, van Doorn MB, Oldenburg RA, van SpaendonckZwarts KY, et al. Renal cancer and pneumothorax risk in Birt-Hogg-Dube syndrome; an analysis of 115 FLCN mutation carriers from 35 BHD families. British journal of cancer. 2011 Dec 6;105(12):1912-9. PubMed PMID: 22146830. Pubmed Central PMCID: 3251884.

4. Toro JR, Glenn G, Duray P, Darling T, Weirich G, Zbar B, et al. Birt-Hogg-Dube syndrome: a novel marker of kidney neoplasia. Archives of dermatology. 1999 Oct;135(10):1195-202. PubMed PMID: 10522666.

5. Menko FH, van Steensel MA, Giraud S, Friis-Hansen L, Richard S, Ungari S, et al. BirtHogg-Dube syndrome: diagnosis and management. The lancet oncology. 2009 Dec;10(12):1199-206. PubMed PMID: 19959076.

6. Kluger N, Giraud S, Coupier I, Avril MF, Dereure O, Guillot B, et al. Birt-Hogg-Dube syndrome: clinical and genetic studies of 10 French families. The British journal of dermatology. 2010 Mar;162(3):527-37. PubMed PMID: 19785621.

7. Nickerson ML, Warren MB, Toro JR, Matrosova V, Glenn G, Turner ML, et al. Mutations in a novel gene lead to kidney tumors, lung wall defects, and benign tumors of the hair follicle in patients with the Birt-Hogg-Dube syndrome. Cancer cell. 2002 Aug;2(2):15764. PubMed PMID: 12204536.

8. Hong SB, Oh H, Valera VA, Stull J, Ngo DT, Baba M, et al. Tumor suppressor FLCN inhibits tumorigenesis of a FLCN-null renal cancer cell line and regulates expression of key molecules in TGF-beta signaling. Molecular cancer. 2010;9:160. PubMed PMID: 20573232. Pubmed Central PMCID: 2907329.

9. Hudon V, Sabourin S, Dydensborg AB, Kottis V, Ghazi A, Paquet M, et al. Renal tumour suppressor function of the Birt-Hogg-Dube syndrome gene product folliculin. Journal of medical genetics. 2010 Mar;47(3):182-9. PubMed PMID: 19843504.

10. Baba M, Hong SB, Sharma N, Warren MB, Nickerson ML, Iwamatsu A, et al. Folliculin encoded by the BHD gene interacts with a binding protein, FNIP1, and AMPK, and is involved in AMPK and mTOR signaling. Proceedings of the National Academy of Sciences of the United States of America. 2006 Oct 17;103(42):15552-7. PubMed PMID: 17028174. Pubmed Central PMCID: 1592464.

11. Baba M, Furihata M, Hong SB, Tessarollo L, Haines DC, Southon E, et al. Kidneytargeted Birt-Hogg-Dube gene inactivation in a mouse model: Erk1/2 and Akt-mTOR activation, cell hyperproliferation, and polycystic kidneys. Journal of the National Cancer Institute. 2008 Jan 16;100(2):140-54. PubMed PMID: 18182616. Pubmed Central PMCID: 2704336.

12. Hartman TR, Nicolas E, Klein-Szanto A, Al-Saleem T, Cash TP, Simon MC, et al. The role of the Birt-Hogg-Dube protein in mTOR activation and renal tumorigenesis. Oncogene. 2009 Apr 2;28(13):1594-604. PubMed PMID: 19234517. Pubmed Central PMCID: 2664853. 
13. Hasumi Y, Baba M, Ajima R, Hasumi H, Valera VA, Klein ME, et al. Homozygous loss of BHD causes early embryonic lethality and kidney tumor development with activation of mTORC1 and mTORC2. Proceedings of the National Academy of Sciences of the United States of America. 2009 Nov 3;106(44):18722-7. PubMed PMID: 19850877. Pubmed Central PMCID: 2765925.

14. Preston RS, Philp A, Claessens T, Gijezen L, Dydensborg AB, Dunlop EA, et al. Absence of the Birt-Hogg-Dube gene product is associated with increased hypoxia-inducible factor transcriptional activity and a loss of metabolic flexibility. Oncogene. 2011 Mar 10;30(10):1159-73. PubMed PMID: 21057536. Pubmed Central PMCID: 3787473.

15. Hong SB, Oh H, Valera VA, Baba M, Schmidt LS, Linehan WM. Inactivation of the FLCN tumor suppressor gene induces TFE3 transcriptional activity by increasing its nuclear localization. PloS one. 2010;5(12):e15793. PubMed PMID: 21209915. Pubmed Central PMCID: 3012117.

16. Cash TP, Gruber JJ, Hartman TR, Henske EP, Simon MC. Loss of the Birt-Hogg-Dube tumor suppressor results in apoptotic resistance due to aberrant TGFbeta-mediated transcription. Oncogene. 2011 Jun 2;30(22):2534-46. PubMed PMID: 21258407. Pubmed Central PMCID: 3109270.

17. Gaur K, Li J, Wang D, Dutta P, Yan SJ, Tsurumi A, et al. The Birt-Hogg-Dube tumor suppressor Folliculin negatively regulates ribosomal RNA synthesis. Human molecular genetics. 2013 Jan 15;22(2):284-99. PubMed PMID: 23077212. Pubmed Central PMCID: 3526160.

18. Esteban MA, Harten SK, Tran MG, Maxwell PH. Formation of primary cilia in the renal epithelium is regulated by the von Hippel-Lindau tumor suppressor protein. Journal of the American Society of Nephrology : JASN. 2006 Jul;17(7):1801-6. PubMed PMID: 16775032.

19. Hartman TR, Liu D, Zilfou JT, Robb V, Morrison T, Watnick T, et al. The tuberous sclerosis proteins regulate formation of the primary cilium via a rapamycin-insensitive and polycystin 1-independent pathway. Human molecular genetics. 2009 Jan 1;18(1):151-63. PubMed PMID: 18845692. Pubmed Central PMCID: 2644647.

20. Ishikawa H, Marshall WF. Ciliogenesis: building the cell's antenna. Nature reviews Molecular cell biology. 2011 Apr;12(4):222-34. PubMed PMID: 21427764.

21. Roy S. The motile cilium in development and disease: emerging new insights. BioEssays : news and reviews in molecular, cellular and developmental biology. 2009 Jul;31(7):694-9. PubMed PMID: 19492356.

22. Patel V, Li L, Cobo-Stark P, Shao X, Somlo S, Lin F, et al. Acute kidney injury and aberrant planar cell polarity induce cyst formation in mice lacking renal cilia. Human molecular genetics. 2008 Jun 1;17(11):1578-90. PubMed PMID: 18263895. Pubmed Central PMCID: 3150596.

23. Hildebrandt F, Benzing T, Katsanis N. Ciliopathies. The New England journal of medicine. 2011 Apr 21;364(16):1533-43. PubMed PMID: 21506742. Pubmed Central PMCID: 3640822.

24. Fischer E, Pontoglio M. Planar cell polarity and cilia. Seminars in cell \& developmental biology. 2009 Oct;20(8):998-1005. PubMed PMID: 19815086.

25. McNeill H. Planar cell polarity and the kidney. Journal of the American Society of Nephrology : JASN. 2009 Oct;20(10):2104-11. PubMed PMID: 19762494.

26. Montani M, Heinimann K, von Teichman A, Rudolph T, Perren A, Moch H. VHL-gene deletion in single renal tubular epithelial cells and renal tubular cysts: further evidence for a cyst-dependent progression pathway of clear cell renal carcinoma in von Hippel-Lindau disease. The American journal of surgical pathology. 2010 Jun;34(6):806-15. PubMed PMID: 20431476. 
27. Basten SG, Willekers S, Vermaat JS, Slaats GG, Voest EE, van Diest PJ, et al. Reduced cilia frequencies in human renal cell carcinomas versus neighboring parenchymal tissue. Cilia. 2013;2(1):2. PubMed PMID: 23369289. Pubmed Central PMCID: 3564780.

28. Kim J, Dabiri S, Seeley ES. Primary cilium depletion typifies cutaneous melanoma in situ and malignant melanoma. PloS one. 2011;6(11):e27410. PubMed PMID: 22096570. Pubmed Central PMCID: 3214062.

29. van Asselt SJ, de Vries EG, van Dullemen HM, Brouwers AH, Walenkamp AM, Giles RH, et al. Pancreatic cyst development: insights from von Hippel-Lindau disease. Cilia. 2013;2(1):3. PubMed PMID: 23384121. Pubmed Central PMCID: 3579754.

30. Bezginov A, Clark GW, Charlebois RL, Dar VU, Tillier ER. Coevolution reveals a network of human proteins originating with multicellularity. Molecular biology and evolution. 2013 Feb;30(2):332-46. PubMed PMID: 22977115. Pubmed Central PMCID: 3548307.

31. Egeberg DL, Lethan M, Manguso R, Schneider L, Awan A, Jorgensen TS, et al. Primary cilia and aberrant cell signaling in epithelial ovarian cancer. Cilia. 2012;1(1):15. PubMed PMID: 23351307. Pubmed Central PMCID: 3555760.

32. Menko FH, Johannesma PC, van Moorselaar RJ, Reinhard R, van Waesberghe JH, Thunnissen E, et al. A de novo FLCN mutation in a patient with spontaneous pneumothorax and renal cancer; a clinical and molecular evaluation. Familial cancer. 2013 Sep;12(3):373-9. PubMed PMID: 23264078.

33. Kouchi M, Okimoto K, Matsumoto I, Tanaka K, Yasuba M, Hino O. Natural history of the Nihon (Bhd gene mutant) rat, a novel model for human Birt-Hogg-Dube syndrome. Virchows Archiv : an international journal of pathology. 2006 Apr;448(4):463-71. PubMed PMID: 16447066.

34. Sang L, Miller JJ, Corbit KC, Giles RH, Brauer MJ, Otto EA, et al. Mapping the NPHP-JBTSMKS protein network reveals ciliopathy disease genes and pathways. Cell. 2011 May 13;145(4):513-28. PubMed PMID: 21565611. Pubmed Central PMCID: 3383065.

35. Takagi Y, Kobayashi T, Shiono M, Wang L, Piao X, Sun G, et al. Interaction of folliculin (Birt-Hogg-Dube gene product) with a novel Fnip1-like (FnipL/Fnip2) protein. Oncogene. 2008 Sep 11;27(40):5339-47. PubMed PMID: 18663353.

36. Singh SR, Zhen W, Zheng Z, Wang H, Oh SW, Liu W, et al. The Drosophila homolog of the human tumor suppressor gene BHD interacts with the JAK-STAT and Dpp signaling pathways in regulating male germline stem cell maintenance. Oncogene. 2006 Sep 28;25(44):5933-41. PubMed PMID: 16636660.

37. Fisch C, Dupuis-Williams P. Ultrastructure of cilia and flagella - back to the future! Biology of the cell / under the auspices of the European Cell Biology Organization. 2011 Jun;103(6):249-70. PubMed PMID: 21728999.

38. Adams NA, Awadein A, Toma HS. The retinal ciliopathies. Ophthalmic genetics. 2007 Sep;28(3):113-25. PubMed PMID: 17896309.

39. Delaval B, Bright A, Lawson ND, Doxsey S. The cilia protein IFT88 is required for spindle orientation in mitosis. Nature cell biology. 2011 Apr;13(4):461-8. PubMed PMID: 21441926. Pubmed Central PMCID: 3073523.

40. Wei C, Bhattaram VK, Igwe JC, Fleming E, Tirnauer JS. The LKB1 tumor suppressor controls spindle orientation and localization of activated AMPK in mitotic epithelial cells. PloS one. 2012;7(7):e41118. PubMed PMID: 22815934. Pubmed Central PMCID: 3399794.

41. Thoma CR, Matov A, Gutbrodt KL, Hoerner CR, Smole Z, Krek W, et al. Quantitative image analysis identifies pVHL as a key regulator of microtubule dynamic instability. The Journal of cell biology. 2010 Sep 20;190(6):991-1003. PubMed PMID: 20855504. Pubmed Central PMCID: 3101603.

42. Ryan MJ, Johnson G, Kirk J, Fuerstenberg SM, Zager RA, Torok-Storb B. HK-2: an immortalized proximal tubule epithelial cell line from normal adult human kidney. Kidney international. 1994 Jan;45(1):48-57. PubMed PMID: 8127021. 
43. Yang Y, Padilla-Nash HM, Vira MA, Abu-Asab MS, Val D, Worrell R, et al. The UOK 257 cell line: a novel model for studies of the human Birt-Hogg-Dube gene pathway. Cancer genetics and cytogenetics. 2008 Jan 15;180(2):100-9. PubMed PMID: 18206534. Pubmed Central PMCID: 2440670.

44. Lancaster MA, Schroth J, Gleeson JG. Subcellular spatial regulation of canonical Wnt signalling at the primary cilium. Nature cell biology. 2011 Jun;13(6):700-7. PubMed PMID: 21602792. Pubmed Central PMCID: 3107376.

45. Lancaster MA, Gleeson JG. Cystic kidney disease: the role of Wnt signaling. Trends in molecular medicine. 2010 Aug;16(8):349-60. PubMed PMID: 20576469. Pubmed Central PMCID: 2919646.

46. Plotnikova OV, Golemis EA, Pugacheva EN. Cell cycle-dependent ciliogenesis and cancer. Cancer research. 2008 Apr 1;68(7):2058-61. PubMed PMID: 18381407. Pubmed Central PMCID: 2546565.

47. MacDonald BT, Tamai K, He X. Wnt/beta-catenin signaling: components, mechanisms, and diseases. Developmental cell. 2009 Jul;17(1):9-26. PubMed PMID: 19619488. Pubmed Central PMCID: 2861485.

48. Wallingford JB, Mitchell B. Strange as it may seem: the many links between Wnt signaling, planar cell polarity, and cilia. Genes \& development. 2011 Feb 1;25(3):20113. PubMed PMID: 21289065. Pubmed Central PMCID: 3034894.

49. Rookmaaker MB, van Gerven HAJM, Goldschmeding R, Boer WH. Solid renal tumours of collecting duct origin in patients on chronic lithium therapy. Clinical Kidney Journal. 2012 October 1, 2012;5(5):412-5.

50. Hedgepeth CM, Conrad LJ, Zhang J, Huang HC, Lee VM, Klein PS. Activation of the Wnt signaling pathway: a molecular mechanism for lithium action. Developmental biology. 1997 May 1;185(1):82-91. PubMed PMID: 9169052.

51. Nookala RK, Langemeyer L, Pacitto A, Ochoa-Montano B, Donaldson JC, Blaszczyk BK, et al. Crystal structure of folliculin reveals a hidDENN function in genetically inherited renal cancer. Open biology. 2012 Aug;2(8):120071. PubMed PMID: 22977732. Pubmed Central PMCID: 3438538.

52. Chua CE, Lim YS, Tang BL. Rab35--a vesicular traffic-regulating small GTPase with actin modulating roles. FEBS letters. 2010 Jan 4;584(1):1-6. PubMed PMID: 19931531.

53. Smith KR, Kieserman EK, Wang PI, Basten SG, Giles RH, Marcotte EM, et al. A role for central spindle proteins in cilia structure and function. Cytoskeleton. 2011 Feb;68(2):112-24. PubMed PMID: 21246755.

54. Toro JR, Wei MH, Glenn GM, Weinreich M, Toure O, Vocke C, et al. BHD mutations, clinical and molecular genetic investigations of Birt-Hogg-Dube syndrome: a new series of 50 families and a review of published reports. Journal of medical genetics. 2008 Jun;45(6):321-31. PubMed PMID: 18234728. Pubmed Central PMCID: 2564862.

55. Woodward ER, Ricketts C, Killick P, Gad S, Morris MR, Kavalier F, et al. Familial nonVHL clear cell (conventional) renal cell carcinoma: clinical features, segregation analysis, and mutation analysis of FLCN. Clinical cancer research : an official journal of the American Association for Cancer Research. 2008 Sep 15;14(18):5925-30. PubMed PMID: 18794106.

56. Vocke CD, Yang Y, Pavlovich CP, Schmidt LS, Nickerson ML, Torres-Cabala CA, et al. High frequency of somatic frameshift BHD gene mutations in Birt-Hogg-Dubeassociated renal tumors. Journal of the National Cancer Institute. 2005 Jun 15;97(12):931-5. PubMed PMID: 15956655.

57. Edelman EA, Girirajan S, Finucane B, Patel PI, Lupski JR, Smith AC, et al. Gender, genotype, and phenotype differences in Smith-Magenis syndrome: a meta-analysis of 105 cases. Clinical genetics. 2007 Jun;71(6):540-50. PubMed PMID: 17539903. 


\section{CHAPTER 2}

58. Albuquerque C, Breukel C, van der Luijt R, Fidalgo P, Lage P, Slors FJ, et al. The 'justright' signaling model: APC somatic mutations are selected based on a specific level of activation of the beta-catenin signaling cascade. Human molecular genetics. 2002 Jun 15;11(13):1549-60. PubMed PMID: 12045208.

59. Lefievre L, Nash K, Mansell S, Costello S, Punt E, Correia J, et al. 2-APB-potentiated channels amplify CatSper-induced $\mathrm{Ca}(2+)$ signals in human sperm. The Biochemical journal. 2012 Dec 1;448(2):189-200. PubMed PMID: 22943284. Pubmed Central PMCID: 3492921.

60. Hu J, Dong A, Fernandez-Ruiz V, Shan J, Kawa M, Martinez-Anso E, et al. Blockade of Wnt signaling inhibits angiogenesis and tumor growth in hepatocellular carcinoma. Cancer research. 2009 Sep 1;69(17):6951-9. PubMed PMID: 19690140. 


\section{CHAPTER 3}

\section{The role of flcn in zebrafish embryonic development}

In preparation

Emma Kenyon*, Monique N.H. Luijten*, Harmeet Gill, Nan Li, Matthew Rawlings, James Bull, Yavor Hadzhiev, Maurice A.M. van Steensel, Eamonn R. Maher and Ferenc Müller

* These authors contributed equally to the manuscript 


\section{Abstract}

Birt-Hogg-Dubé syndrome (BHD) is a rare, hereditary disorder, which predisposes individuals to development of benign facial tumours called fibrofolliculomas, pneumothorax and renal cancer. BHD is caused by heterozygous mutations in the gene encoding folliculin (FLCN), an evolutionarily conserved protein of currently unknown function. Folliculin is required for embryonic development, but the protein's exact developmental role is difficult to examine in available animal models as homozygous flcn knockout causes embryonic lethality in mammals.

Therefore, we generated a zebrafish loss of function BHD model. The zebrafish FLCN ortholog is expressed during all stages of embryonic development, with increased expression in the fin bud, somites, eye and regions of the brain as determined by whole-mount in situ hybridization. Knockdown of zebrafish Flcn using morpholinos results in developmental arrest 18 hours post fertilisation (hpf) and disrupted brain morphogenesis, defects in tail circulation and the yolk at 48 hpf. This might be a result of cell cycle defects. In zFucci flcn morphants a gradual increase in the amount of G1 cells was detected accompanied by a decreased population of S, G2 and M cells in the retina and compartments of the brain. Together, these results point towards a critical role for flcn in development. 


\section{Introduction}

Birt-Hogg-Dubé syndrome (BHD) is a dominantly inherited familial cancer syndrome characterised by the development of benign skin fibrofolliculomas, multiple lung cysts, spontaneous pneumothorax and kidney cysts with susceptibility to renal cell carcinoma $(1,2)$. The syndrome results from heterozygous, inactivating mutations in the gene encoding folliculin (FLCN) (3-6). FLCN has been implicated in the regulation of various signalling pathways and cellular processes including cellular metabolism through mTOR, AMPK and HIF1 $\alpha$, transcriptional regulation, JAK-STAT signalling, cell adhesion, ciliogenesis, lysosomal biogenesis and autophagy (7-23). However, several groups have generated conflicting data on the consequences of FLCN deficiency and how these lead to the clinical manifestations associated with BHD is not clear.

Investigations of a whole organism model system will provide important insights into the nature and evolutionary conservation of BHD-related effects on target signalling pathways. Studies using experimental animals have indicated that FLCN is required for embryogenesis, but the exact developmental role of the protein is difficult to examine in available animal models as homozygous FLCN knockout causes early embryonic lethality in mammals $(8,9,24-28)$.

Due to their ex vivo development, enabling easy manipulation and visualisation from the single cell stage, and due to their large offspring production, zebrafish (Danio rerio) are very well suited to study the pathophysiology of developmental defects. In addition, zebrafish have very similar tumours (benign as well as malignant) to humans (29) showing a high degree of conservation in gene expression signatures (30). Moreover, zebrafish are the only vertebrate in which it is possible to perform large-scale genetic screens that could be targeted to conserved cancer pathways. For example stable transgenic lines are available that can provide a platform for ontogeny and drug screens (31). Importantly, the genes involved in the differentiation and proliferation pathways of the vertebrate embryo are often the same that are affected in cancer (reviewed in (32)), thus developmental defects and genetic mechanisms uncovered by embryogenetic tools often provide valuable insights into the components of genetic pathways acting in cancer.

Therefore, we generated a zebrafish loss of function model for BHD. As a first approach we characterise flcn morpholino knockdown in zebrafish. We then reconcile the observed phenotype with expression analysis of the flcn transcripts in zebrafish development. 


\section{Results}

Isolation and expression of the zebrafish flcn gene

Based on a sequence search in the zebrafish genome, we identified a flcn homolog with very high sequence similarity to the human FLCN gene (ENSDARG00000062385 Ensembl Zv9) (Figure 1A). To address when and where flcn is expressed we utilized our published (33) genome level analysis of the zebrafish transcriptome (cap analysis of gene expression (CAGE) and RNA sequencing). Zebrafish flcn is expressed maternally, is present in the premidblastula embryo at high levels until $1 \mathrm{k}$-cell stage and then falls by dome stage. flcn is transcribed by the embryo at least up to larval stages (Figure 1B).

We cloned the full open reading frame of the zebrafish flcn gene product by RTPCR from 3 day post fertilisation (dpf) zebrafish larvae and verified its integrity by sequencing and alignment to the zebrafish genome. To identify the flcn expression dynamics during several stages of embryo development we conducted whole mount in situ hybridization At the start of gastrulation, flcn expression can been seen at low levels over the whole embryo (Figure 1C) and this expression remains present at subsequent developmental stages. Additionally, at 4 somite stage flcn shows increased expression in the region of the tail bud (Figure 1D arrow). At 24 hpf increased expression is detected in the myotome border/somatic furrow, eye, hatching gland (Figure 1E arrowhead), fin bud (Figure 1E arrow), the tectum (Figure $1 \mathrm{~F}$ arrowhead) and the telencephalon (Figure $1 \mathrm{~F}$ arrow). At $50 \mathrm{hpf}$ there is pronounced expression in a thin layer of cells in the posterior tectum (Figure 1G arrow, I and K) and fin (Figure 1G). Expression of flcn was compared to whole mount in situ hybridization of proliferating cell nuclear antigen (pcna), expressed in cycling cells (Figure $1 \mathrm{H}, \mathrm{J}$ and $\mathrm{L}$ ). The overlapping expression in the posterior tectum can be confirmed by fluorescent double in situ hybridization of flcn (Figure $1 \mathrm{~K}$ and $\mathrm{M}$ ) and pcna (Figure $1 \mathrm{~L}$ and $\mathrm{M}$ ).

Figure 1 - Conservation and expression of zebrafish flch

(A) Comparative alignment of human FLCN and zebrafish Flcn protein sequences using Clustal Omega $(34,35)$, manually edited using Jalview (36) and displayed with Clustal X colour scheme. (B) Graphs showing CAGE transcription start site values (upper panel) and RNA sequencing reads per kb per million (RPMK) values (lower panel) for zebrafish flcn at indicated stages. (C-G) Whole mount in situ hybridization of flcn expression at (C) shield stage; (D) 4 somite stage, with increased expression in the region of the tail bud (arrow); (E) $28 \mathrm{hpf}$, showing more pronounced expression in the fore brain and hind brain areas, hatching gland (arrowhead) and the fin bud (arrow); (F) 28 hpf, showing flcn expression in the tectum (arrow) and telencephalon (arrowhead) and (G) 50 hpf, showing flcn expression in the brain and retina. (H) $50 \mathrm{hpf}$ embryo showing pcna expression in the retina and brain. (I) flcn expression in the posterior tectum at $50 \mathrm{hpf}$ (arrow). (J) pcna expression in the posterior tectum at $50 \mathrm{hpf}$ (arrow). (K-M) Head region of a $50 \mathrm{hpf}$ embryo showing expression of flcn (K green) and pcna ( $\mathrm{L}$ red) in the posterior tectum. (M) Merge of $\mathrm{K}$ and $\mathrm{L}(\mathrm{n}=20$ embryos for all WMISH). 


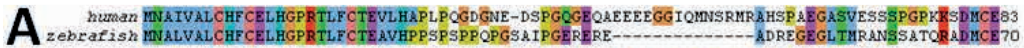

human GCRSL zebraf is $h$ GCRSLP ASHP GFUSVDGETGIRYLSHQHPRQPQLF SWVQACVRSLSCEVCP GREGP IF FGDEQHGFVF SHTFF IKDNL גRGFQ15

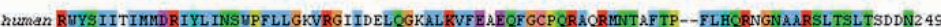
zebraf is $h$ BWYS IVVVAMDR IYL INSUPFLLRHL RL TIQSLCNTALKVF DSEQCVCPORALRMNSTFSPAVF PHCRSGNAARSLTSLTOHPN238

human LWACLHTSF AWLLEACGSRLTEKLLEGAPTEDTLVOMEKLADFLEEFSSWDNSEAEEEEKAPVLPESTEGRELTQGPAESSSLS333

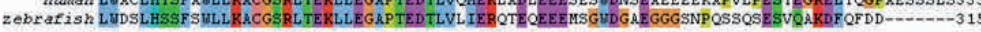

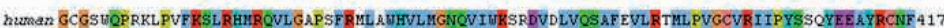

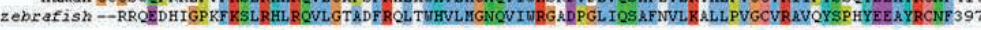

human LGLSPHVOIPPHVLSSEF AVIVEVHAMARTSTLHPVGCEDDQSLSKYEFVVTSGSPVAL̆DRVGPTILNKIEAAL TNONLSVDVVD 501 zebraf is h LGLSPDVP IP AHVSSSEF SVLVDVLSAERGCUNPVSD DD--ILSLYOF HISS ANT-QRTDKGPTLLNKLEVALSNENLSVEVVS478

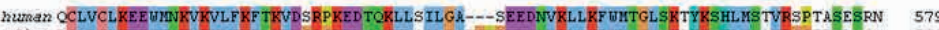
zebraf ish LCLLCLKEEWINKVKVLF KF SKVD GRGKEDTCKVL ALLGATGPGEEDNVRLLKFWITGLSKLYKSHL HT AVRGGERTLSQ- 550

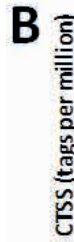
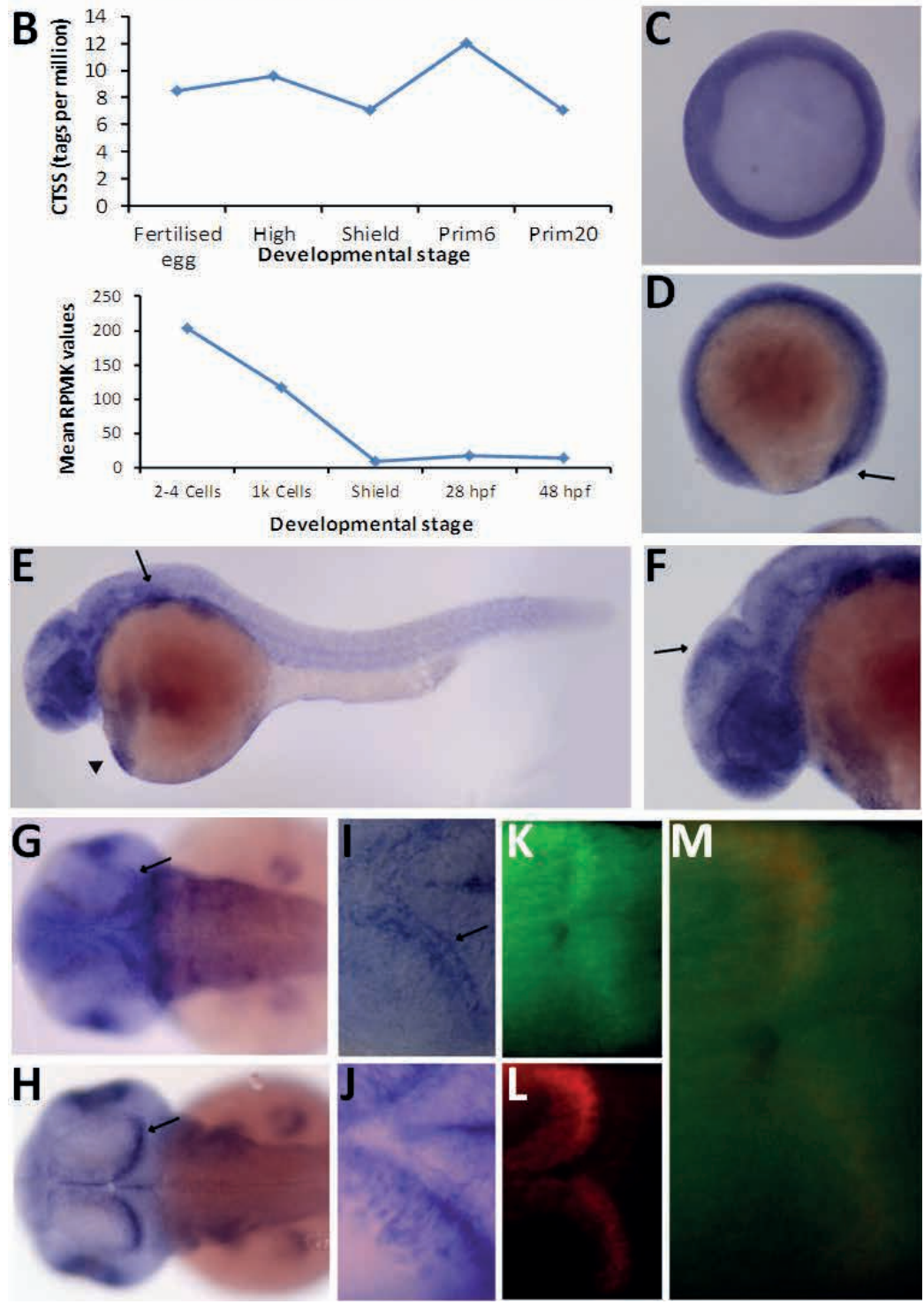


\section{Morpholino knockdown suggests embryonic functions of folliculin}

Morpholino antisense oligonucleotides (MO) were designed to block the start site or splicing sites of flcn and thus to knockdown Flcn protein expression (Figure 2A). As splice morpholinos are designed to interfere with splicing of pre-mRNA, efficiency of morpholino-induced knockdown was assessed by RT-PCR on total RNA extracted from a pool of injected 48 hpf embryos. In embryos injected with flcn splice 1 morpholino the RT-PCR product was larger than that seen in the control mismatch morpholino-injected embryos, suggesting that an 88 bp intron was retained (Figure S1A). Retention of this intron leads to a premature stop. In embryos injected with flcn splice 2 morpholino no product was observed when compared to the mismatch morpholino, indicating inclusion of a $3.5 \mathrm{~kb}$ intron (Figure 2B).

Knockdown of the zebrafish flcn gene by microinjection of either $100 \mu \mathrm{M}$ of splice 2 (Figure 2D and F), $600 \mu \mathrm{M}$ of ATG (Figure 2H) or $100 \mu \mathrm{M}$ of splice 1 (Figure S1C) flcn morpholino into wild type zebrafish embryos resulted in a clear and reproducible phenotype. At 18 hours post fertilisation (hpf) embryos injected with flcn morpholino appeared to show developmental arrest when compared with mismatch controls. At 24 hpf experimental embryos showed a two-hour delay in development, exhibited cell death in the brain and trunk with U-shaped somites and undulating notochord in the tail (Figure 2D) when compared to mismatch injected embryos (Figure 2C). At $48 \mathrm{hpf}$ the phenotype was characterised by brain oedema and problems with tail circulation (Figure $2 \mathrm{~F}$ and $\mathrm{H}$ ). There was also a larger yolk and thinner yolk extension (Figure $2 \mathrm{~F}$ and $\mathrm{H}$ ) when compared to controls (Figure 2E and G) which were indistinguishable from the uninjected embryos. To better visualise the hydrocephalus we injected phenol red into the fourth ventricle of the brain of mismatch and flcn morpholino injected embryos. After 10 minutes flcn morphants showed phenol red throughout the embryo (Figure 2J) while mismatch injected embryos retained phenol red in the fourth ventricle (Figure 2I). We also injected high molecular weight rhodamine into the fourth ventricle of the brain of mismatch and flcn morpholino injected embryos as described by Ferrante et al. (37). The rhodamine was restricted to the fourth ventricle of mismatch injected embryos (Figure $2 \mathrm{~K}$ ) while in the flcn morphants rhodamine was detected from the fourth ventricle to the forebrain (Figure 2L).

To confirm that the morphant phenotype is caused by reduced flcn expression, we aimed to perform rescue experiments by injection of the flcn splice 2 morpholino and full-length flcn RNA (Figure 2M). To do so, the full open reading frame of zebrafish flcn was cloned into the pCS2+ vector and RNA was produced in vitro. As injection of increasing concentrations of flcn RNA resulted in embryos of reduced size showing cyclopia, heart oedema and circulatory defects (Figure $2 \mathrm{M}, \mathrm{R}$ and S), rescue of morpholino knockdown was assessed by abolishment of the hydrocephalus phenotype. Increasing the amount of flcn RNA in embryos injected with flcn splice 2 morpholino resulted in a decreased percentage of embryos 
showing severe hydrocephalus (Figure 2P) and an increase in the percentage of embryos with mild or no hydrocephalus (Figure $2 \mathrm{~N}$ and 0 ).

Together, these results strongly suggest that the flcn morpholinos are specific to the flcn transcripts; that flcn levels need to be tightly regulated and that flcn is required for the development of the zebrafish embryo and may be particularly important in the brain.
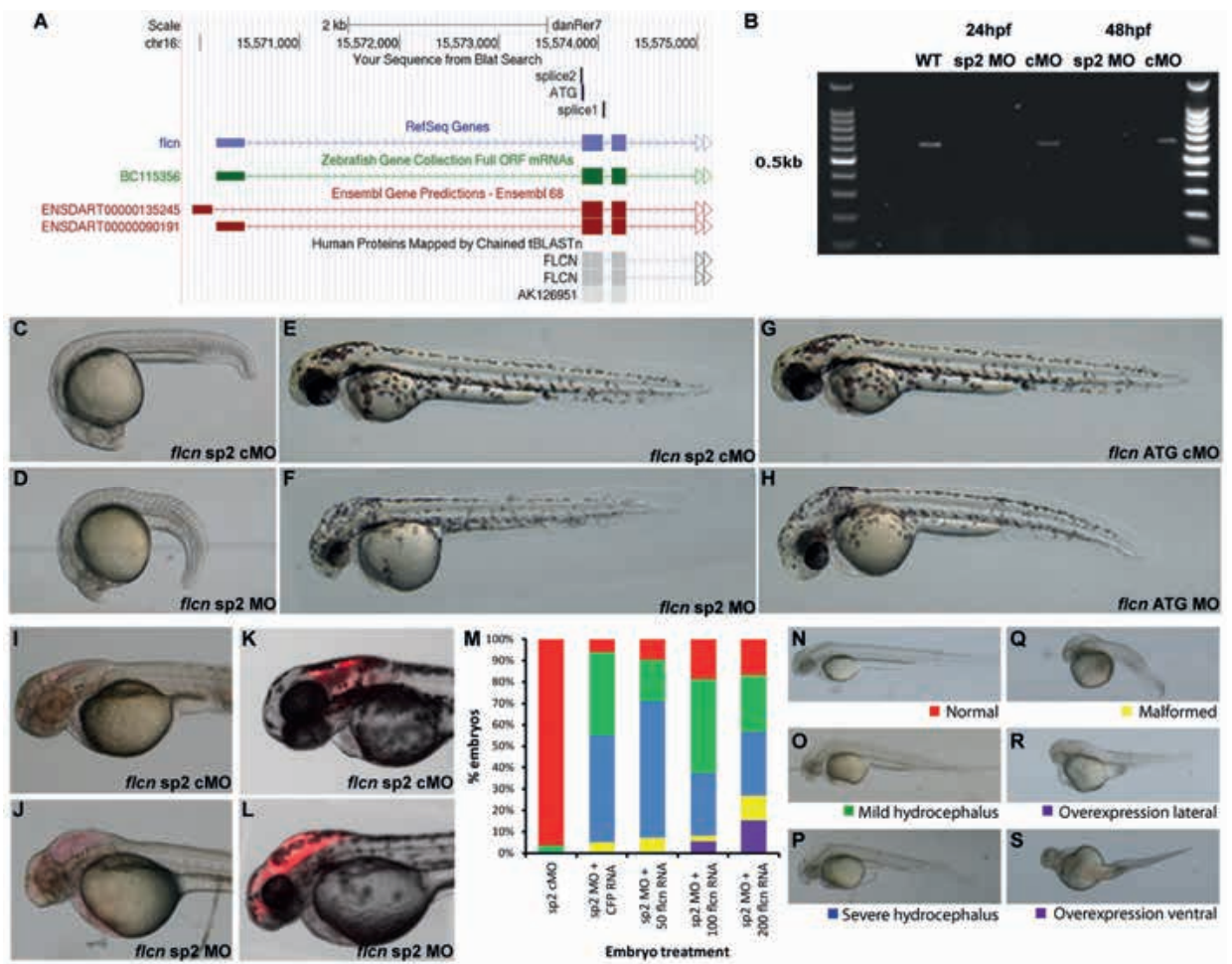

Figure 2 - Knockdown of flcn in zebrafish embryos.

(A) Schematic from the UCSC genome browser showing the binding sites of the flcn morpholinos. (B) Electrophoresis gel comparing the size of an amplified flcn transcript in wild type (WT) embryos or embryos injected with mismatch morpholino (cMO) or flcn splice 2 morpholino (sp2 MO). (C-H) Phenotype of $24 \mathrm{hpf}$ embryos injected with $100 \mu \mathrm{M}$ splice 2 mismatch (C) or flcn morpholino (D) and 48 hpf embryos injected with $100 \mu \mathrm{M}$ control splice 2 mismatch (E), $100 \mu \mathrm{M}$ flcn morpholino (F), $600 \mu \mathrm{M}$ ATG mismatch(G) or $600 \mu \mathrm{M}$ flcn ATG morpholino (H). (I-J) Representative 48 hpf PTU-treated embryo injected with splice 2 mismatch (I) or flcn splice 2 morpholino (J) 10 minutes after the injection of a phenol red dye into the fourth ventricle. ( $\mathrm{n}=10$ embryos injected) (K-L) Representative $48 \mathrm{hpf}$ embryo injected with splice 2 mismatch (K) or flcn splice 2 morpholino (L) after injection with $70 \mathrm{kDa}$ rhodamine into the fourth ventricle of the brain. ( $\mathrm{n}=8$ embryos injected). (M-S) Rescue experiments in flcn morphants for hydrocephalus phenotype. (M) Percentage of embryos that show normal, hydrocephalus and overexpression phenotype when injected with combinations of $100 \mu \mathrm{M}$ splice 2 mismatch morpholino, control CFP RNA, $100 \mu \mathrm{M}$ flcn splice 2 morpholino and $100 \mathrm{pg} / \mu \mathrm{l}, 200 \mathrm{pg} / \mu \mathrm{l}$ or $300 \mathrm{pg} / \mu \mathrm{l}$ of flcn RNA. ( $\mathrm{n}=2$ independent injections, minimum of 50 embryos per group per experiment). Representative images are shown for PTU-treated $48 \mathrm{hpf}$ normal embryos (N), mild (O) or severe (P) hydrocephalus, malformed embryos (Q) and the overexpression cyclopia phenotype (R-S). 

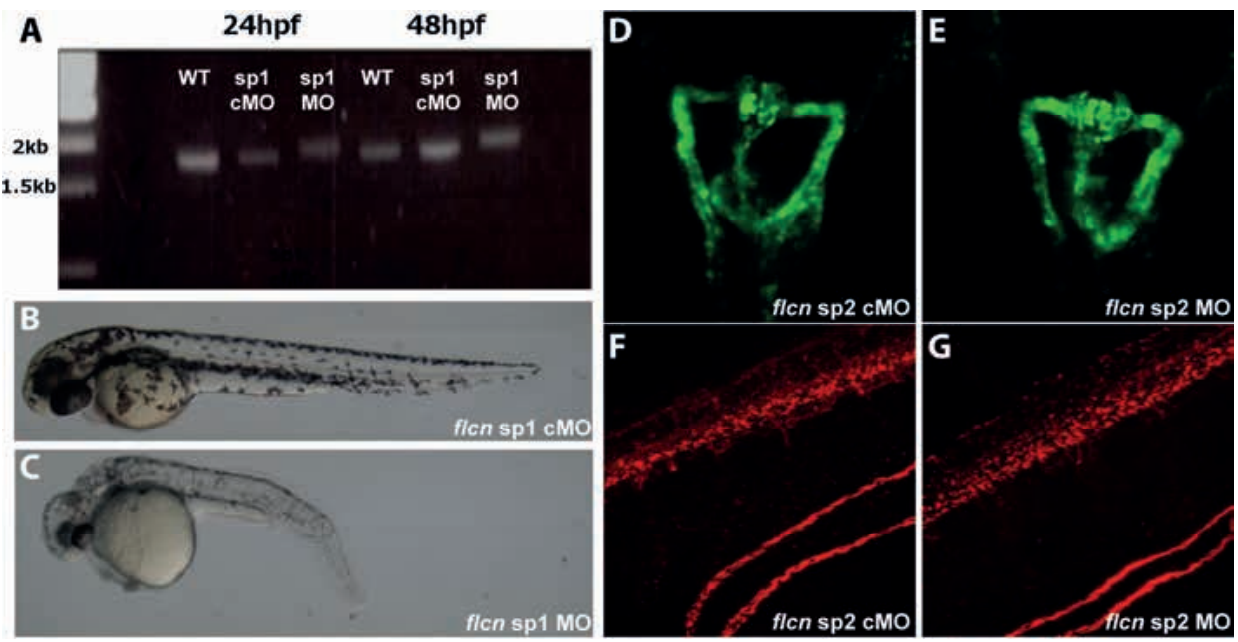

Supplemental Figure S1 - Knockdown of flcn and its effect on cilia and kidney development in embryos.

(A) Electrophoresis gel comparing the size of an amplified flcn transcript in wild type (WT) embryos, embryos injected with mismatch morpholino (sp1 cMO) and embryos injected with flcn splice 1 morpholino (sp1 MO). (B-C) Phenotype of 48 hpf embryo injected with $100 \mu \mathrm{M}$ splice 1 mismatch (B) or $100 \mu \mathrm{M}$ splice 1 flcn morpholino (C). (D-E) Fluorescent imaging of $T g$ (wt1b:EGFP) transgenic zebrafish embryos injected with $100 \mu \mathrm{M}$ splice 2 mismatch (D) or $100 \mu \mathrm{M}$ splice 2 flcn morpholino (E) (n = 8 embryos, 2 independent experiments). (F-G) Acetylated alpha tubulin staining of cilia in $24 \mathrm{hpf}$ embryos injected with $100 \mu \mathrm{M}$ splice 2 mismatch (F) or $100 \mu \mathrm{M}$ splice 2 flcn morpholino (G) (n $=10$ embryos, 2 independent experiments).

flcn morphants do not show defects in kidney or motile cilia in early development.

As BHD patients have an increased risk of developing renal cysts and tumours (2), we decided to study the effect of flcn knockdown on kidney development in zebrafish embryos. The Wilms Tumor-1b EGFP transgenic line (Tg(wt1b:EGFP)) expresses EGFP exclusively in the pronephros and exocrine pancreas (38). Injection of flcn morpholino into $\mathrm{Tg}(w t 1 b: E G F P)$ embryos did not cause any obvious defects in pronephric development at $48 \mathrm{hpf}$ (Figure S1E) when compared with mismatch injected (Figure S1D).

Morpholino knockdown of flcn in zebrafish resulted in a phenotype reminiscent of ciliary gene mutants (39) and morphants (40). In addition, BHD in humans was recently described as a novel ciliopathy (14). However, no difference in pronephric and central canal cilia was observed in 24 hpf embryos injected with flcn morpholino (Figure S1F) when compared with mismatch injected embryos (Figure S1E) after immunofluorescent staining of acetylated alpha tubulin (a known ciliary marker). 
Cell cycle defects in brain development of flcn morphant embryos

The lack of obvious ciliary defects prompted us to examine other cellular processes that might be affected by Flcn deficiency, thereby causing the morphant phenotype. In vitro, FLCN has been implicated in cell cycle regulation via late $\mathrm{S}$ and $\mathrm{G} 2 / \mathrm{M}$ phase and cyclin D1 $(11,41,42)$. In addition, the flcn morphants show developmental arrest and flcn is expressed in the proliferative zone of the brain. Therefore, cell cycle regulation was monitored in zebrafish embryos after flcn knockdown using a novel, powerful in vivo labelling tool.

The zFucci system (43) is composed of two transgenes that fluorescently label G1 (red) and S-M phase (green) nuclei in the living zebrafish embryo at remarkable temporal resolution. The system is based on detecting ubiquitination of the fluorescent fusion proteins Cdt1-Kusabira Orange2 and Geminin-Azami Green. zFucci double transgenic embryos were injected with flcn splice 2 morpholino or mismatch and reporter activities indicative of S-M (green) and G1 (red) phases were monitored. A snapshot of green and red fluorescence was used to evaluate the status of cell cycles around 18 somite stage. This analysis indicated a significant drop in S-M states (Figure 3J) in flcn morphants (Figure 3E, F, H) particularly in the retina and compartments of the brain including the tectum, when compared with mismatch-injected embryos (Figure 3A, B, D). A corresponding increase in G1 cells (Figure 3I) in flcn morpholino-injected embryos (Figure 3G) was also seen when compared with mismatch-injected embryos (Figure 3C).

Time-lapse analysis of the head region (Figure $4 \mathrm{~A}$ yellow box) in flcn morpholino- (Figure 4G, H, I and J) and mismatch- (Fig 4C, D, E, and F) injected embryos over the period from 6 somite to prim-20 stages shows that initially embryos have comparable levels of cells in G1 phase (Figure 4B). As time progresses there is a gradual increase in G1 cells in flcn morphants when compared to mismatch-injected embryos (Figure 4B). 

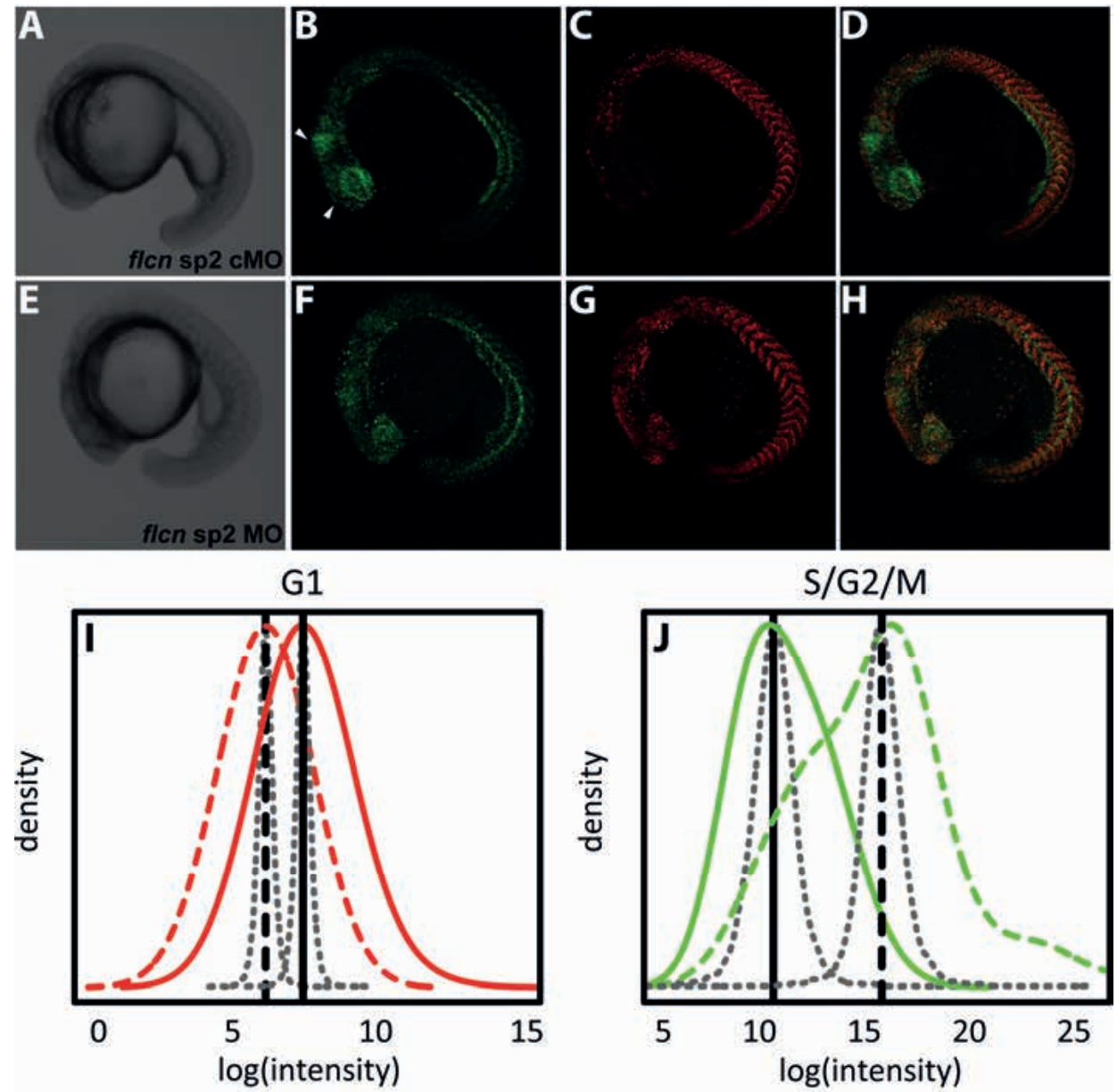

Figure 3 - Cell cycle defects in flcn morphant embryos at 18 somite stage.

(A-D) Zebrafish Fucci embryo injected with splice 2 mismatch morpholino imaged in (A) brightfield and (B) fluorescence microscopy for Geminin-Azami Green (S/G2/M phase) or (C) Cdt1-Kusabira Orange2 (G1). (E-H) Zebrafish Fucci embryo injected with flcn splice 2 morpholino imaged in (E) brightfield and (F) fluorescence microscopy for Geminin-Azami Green or (G) Cdt1-Kusabira Orange2. (D\&H) Merge of Geminin-Azami Green and Cdt1-Kusabira Orange2. (I-J) Data distribution of pixel intensity (log2 (intensity)) of Cdt1-Kusabira Orange2 (I) or Geminin-Azami Green (J) in embryos injected with flcn splice 2 morpholino (solid lines) compared to mismatch (dashed lines). Vertical, black lines indicate means, with parameter confidence distributions shown as curved black lines. 

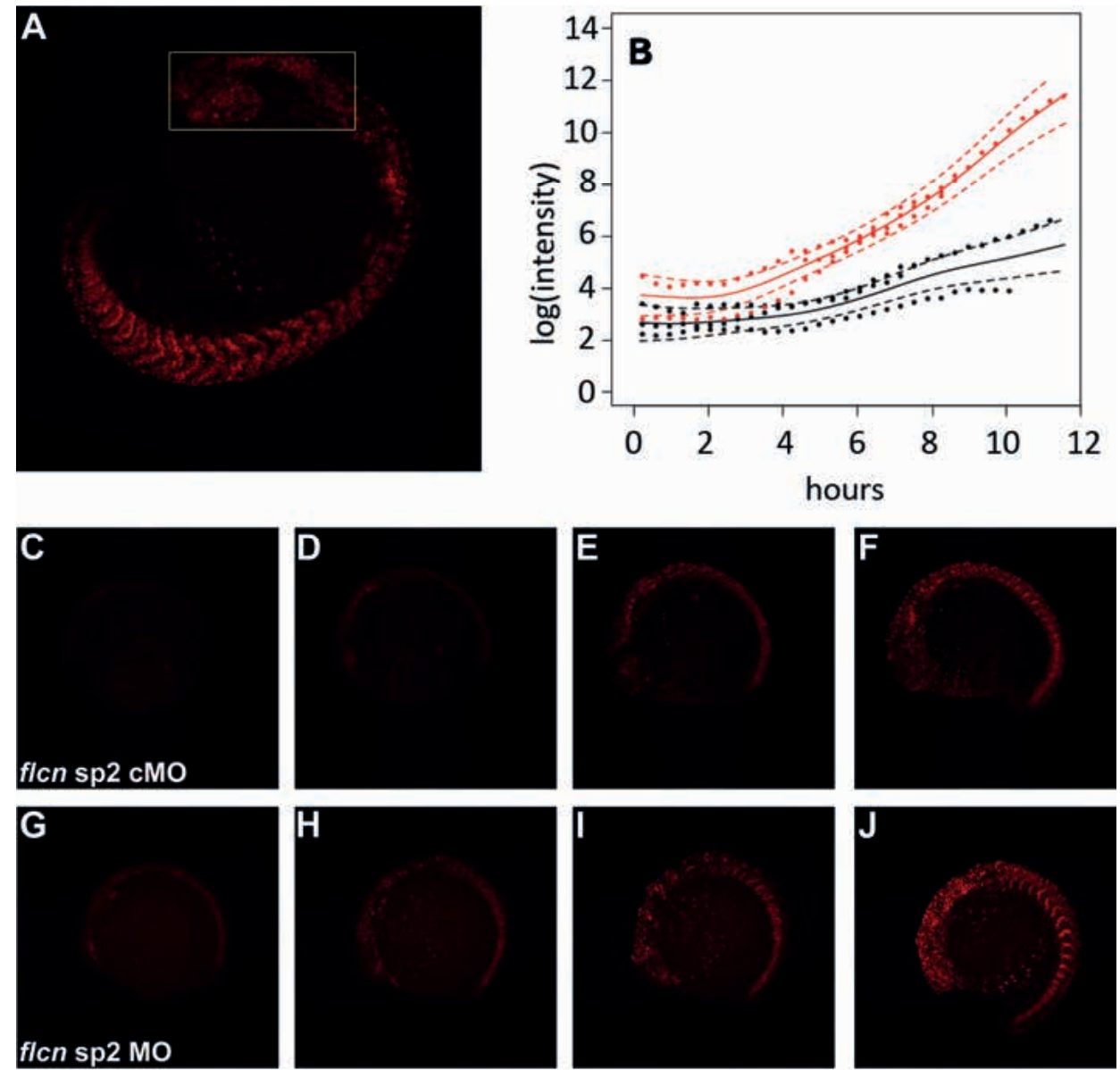

Figure 4 - Cell cycle defects in flcn morphant embryos over time.

(A) Zebrafish Fucci embryo showing fluorescence for Cdt1-Kusabira Orange2 with analysed region of the head indicated by yellow box. (B) Longitudinal time courses of $\log _{10}$ (intensity) of pixels from 3 splice 2 flcn mismatch (black) and 4 splice 2 flcn morpholino (red) injected whole embryos. Frames were taken from bud-stage for splice 2 flcn mismatch and 2 somite stage for splice 2 flcn morpholino injected embryos. Solid lines indicate estimated mean trajectories for each treatment group, with dashed lines denoting parameter 95\% confidence intervals. (C-J) Embryos injected with (C-F) mismatch or (G-J) splice 2 flcn morpholino at bud-stage (C), 2 somite stage (D\&G), 4 somite stage (E\&H), 6 somite stage (I), 10 somite stage (F) or 13 somite stage (J). 


\section{Discussion}

In this paper we show the impact of flcn knockdown on zebrafish development. In particular, we provide evidence that flcn may be required for zebrafish embryonic brain development.

Canine and murine animal models homozygous for FLCN knockout show early embryonic lethality $(8,9,24-28)$. Chen et al. showed that mice homozygous for flcn mutation die between $3.5 \mathrm{dpc}$ and $8.5 \mathrm{dpc}$ (8). Hasumi et al. narrowed down the embryonic lethality to E5.5-E6.5 (24). Most mutant mice lacked an organised epiblast and surrounding visceral endoderm. In embryos that did develop visceral endoderm and ectoderm, these layers had a disorganised structure containing cells with an enlarged cytoplasm and disorientated nuclei (24). In contrast, flcn morphant zebrafish embryos develop normally past gastrulation, with the first signs of disruption occurring when the embryos arrest at $18 \mathrm{hpf}$. By $24 \mathrm{hpf}$ the embryos exhibit cell death in the brain and trunk and by $48 \mathrm{hpf}$ they show brain oedema and problems with tail circulation, as well as a larger fragile yolk and thinner yolk extension. Injection of dyes into the fourth ventricle of the brain showed considerable brain disruption as the dye diffused throughout the brain of embryos injected with flcn morpholino while remaining in the fourth ventricle of those that were injected with the mismatch morpholino.

This phenotype is supported by whole mount in situ hybridization results showing that flcn is expressed in the tectum and telencephalon in zebrafish and the neural tube in mice (24). In mice, Flcn expression was shown at E7.5 in the neural ectoderm of the primitive streak region and in the headfold of the ectoderm. At E9.5, Flcn was expressed almost ubiquitously with a strong signal in the neural tube and optic pit, while at E10.5 Flcn was highly expressed in the branchial arch, forelimb, and hind limb, as well as in the somites. Adult mice showed expression in the heart, pancreas, and prostate with moderate expression in adult brain, kidney, liver and lung (24). This pattern is consistent with the expression of flcn during zebrafish development, where it is seen in the fin bud, somites, eye and regions of the brain. Although loss of function phenotypes in mouse and zebrafish are different, they both point towards a critical role for flcn in the development of both organisms.

In humans, we have recently described BHD as a novel ciliopathy, characterised by cyst development in organs including kidney, lung, liver and pancreas. FLCN localised to motile and non-motile cilia, centrosomes and the mitotic spindle and alteration of FLCN levels caused changes to the onset of ciliogenesis (14). In zebrafish embryos lacking genes important for ciliogenesis, one of the reported phenotypes is hydrocephalus (37), also seen in flcn morphants. We therefore considered that knockdown of flcn in zebrafish embryos could result in defects in the kidney and/or other ciliated structures. However, we have found no evidence for this when we compared the pronephros of $\mathrm{Tg}(w t 1 b: E G F P)$ embryos with and without flcn knockdown or when using immunofluorescent stainings for acetylated 
alpha tubulin (a ciliary marker). It is possible that, although cilia are present in the flcn knockdown embryos and have no obviously abnormal morphology, they may not be functional. Alternatively, FLCN's function in ciliogenesis may be specific to mammals.

Given the lack of obvious ciliary defects we next sought to determine whether the developmental arrest phenotype and brain phenotype were caused by defects in the cell cycle, as flcn is expressed in the growth zone of the brain. To this end, we knocked down flcn in zFucci transgenic embryos, in which cells in the G1 phase of the cell cycle are fluorescently labelled red and cells in S, G2 and M are labelled green (43). We found that at the 18 somite stage there was a significant drop in SM states in flcn morpholino-injected embryos with a corresponding increase in G1 cells. This was particularly evident in the brain, retina and somites of the embryo, which are all areas that have been shown to express flcn in both mice (24) and zebrafish. These data indicate that flcn positively regulates the cell cycle. Conversely, recent in vitro studies suggest that FLCN deficiency enhances cell cycle progression through late $S$ and G2/M phase (42) or through G1 to S phase via regulation of cyclin D1 (41). These discrepancies might be due to species-specific differences or, alternatively, might be related to the time of deficiency. During embryogenesis Flcn may stimulate cell cycle progression, while in differentiated cells it prevents tumour growth by inhibiting cellular proliferation through regulation of cyclin D1 $(41,42)$.

Alternatively, the cell cycle defects might be secondary to other consequences of Flcn knockdown. G1 can only progress if sufficient growth factors and nutrients are present (reviewed (44)). Lui et al. have shown that Drosophila melanogaster with a genomic deletion of the Flcn gene never survive to adulthood and show characteristics of malnutrition (45). The large, fragile yolks and thin yolk extensions in zebrafish lacking Flcn are indicative of defects in nutrient supply and digestive problems (46-48). In vitro, FLCN has been shown to regulate signalling of AMPK, a major sensor of energy status. FLCN directly interacts with AMPK (7) and loss of FLCN was shown to constitutively activate AMPK leading to mitochondrial biogenesis and HIF-driven aerobic glycolysis $(17,18)$. It is therefore possible that the phenotype we see is a result of metabolic defects causing the zebrafish to not properly utilise its yolk.

Similar to the flcn morphants, improper formation of the yolk extension is also seen in zebrafish embryos with knockdown of chimerin Rac-GAPs. These morphants show faster progression through epiboly, which is driven by the yolk syncytial layer (49). Epiboly is regulated by actin cytoskeleton remodelling (50) possibly through Rac. In vitro FLCN has been linked to actin remodelling via interaction with plakophilin-4 (p0071) $(20,21)$. Although the nature of FLCN's involvement in vitro remains unclear as both up- (20) and downregulation (21) of downstream RhoA signalling have been reported following FLCN knockdown, 
development of thin yolk extensions in the flcn morphants might be a result of defective actin remodelling.

A further possibility is the involvement of the Wnt signalling pathways. In three-dimensional culture abnormal expression of FLCN disrupts polarised growth of kidney cells and deregulates canonical Wnt signalling (14). The canonical Wnt pathway regulates cell proliferation by promoting G1 phase progression through downstream effectors including cyclin D1 (reviewed (51)). In zebrafish, overexpression of flcn resulted in an increased number of embryos showing cyclopia, a phenotype seen in embryos lacking nodal and non-canonical Wnt signalling $(52,53)$. With recent studies showing that non-canonical Wnt signalling can inhibit canonical Wnt signalling $(54,55)$ this is an interesting and complex area for further study.

In summary, we show that zebrafish flcn is important for embryonic development with high expression levels in the very early embryo. Our data suggest that flcn may be specifically involved in embryonic brain development, possibly through regulation of the cell cycle.

During the preparation of this manuscript several concerns were raised by the scientific community about the possible off-target effects resulting from morpholino injection. Novel techniques were recently developed that combine DNA recognition proteins with DNA cleavage domains (e.g. the CRISPR/Cas9 system (56-59)), thereby enabling large-scale generation of targeted zebrafish mutant lines. The majority of these lines do not recapitulate the phenotype induced by injection of a morpholino against the same gene, showing milder defects or even complete absence of developmental defects (N. Lawson, personal communication, 2014). These aggravated phenotypes in the morphants can be rescued by co-injection of morpholino and capped mRNA of the gene of interest, which suggests they might have a gene-specific component. It is possible that the microinjection generates a stress response in the zebrafish embryos, thereby worsening the effect of gene deficiency induced by the morpholino. As the developing zebrafish brain seems to be particularly sensitive to off-target effects, specificity of the flcn morphant phenotype could be examined in a zebrafish flcn mutant line generated using these novel techniques. 


\section{Materials and Methods}

Zebrafish husbandry and embryo generation

Zebrafish embryos were obtained from sibling crosses from adult $A B$ fish housed at the fish facility at Birmingham University. Zebrafish were raised and bred and embryos staged following standard protocols $(60,61)$. Stages are described as the approximate number of hours post-fertilisation (hpf) when embryos are raised at $28.5^{\circ} \mathrm{C}$. When needed to prevent pigment formation, embryos were raised in $0.003 \%$ phenylthiocarbamide in E3 medium $(1 \mathrm{mM} \mathrm{NaCl}, 0.17 \mathrm{mM} \mathrm{KCl}, 0.33 \mathrm{mM}$ $\mathrm{MgSO}_{4} .7 \mathrm{H}_{2} \mathrm{O}, 0.33 \mathrm{mM} \mathrm{CaCl}_{2} .2 \mathrm{H}_{2} \mathrm{O}$ ) from tailbud stage.

flcn cDNA cloning and mRNA production

The full open reading frame for zebrafish flcn was cloned from total RNA extracted from pooled $3 \mathrm{dpf} \mathrm{AB}$ wild type embryos using TRIZOL reagent according to manufacturers' instructions. Next, cDNA was produced using M-MLV Reverse Transcriptase, RNase H Minus, Point Mutant (Promega) and oligodT primers (Invitrogen). Zebrafish flcn cDNA was amplified using the following forward primer with BamHI restriction site: AATA GGATCC ATGAACGCTTTAGTTGCCCTG and reverse primer with Xba1 restriction site: AATA TCTAGA CCCGCTTTCAGTCTCTCTCAC and cloned into pCS2+ using BamHI (New England Biolabs) and Xbal (New England Biolabs). Plasmid was verified by sequence analysis. Capped RNA was synthesised using $5 \mu \mathrm{g}$ of NotI linearized flcn DNA using SP6 mMESSAGE mMACHINE kit (Ambion). The RNA was cleaned using GenElute ${ }^{\mathrm{TM}}$ Mammalian Total RNA Miniprep Kit (Sigma) as per manufacturer's instructions Appendix 2.

Microinjection of embryos with RNA and morpholino

Morpholino oligonucleotides (GeneTools) as follows:

FLCN ATG MO

FLCN splice1 MO

FLCN splice1 control MO

FLCN splice2 MO

FLCN splice2 control MO
AGGGCAACTAAAGCGTTCATCTGTG $(8 \mathrm{ng} / \mu \mathrm{l})$

ATGACACTCCCCTCTCGCTCACCTC

ATcACAgTCCCCTgTCGCTgACgTC

CGTTCATCTGGAGGAAACAAACATA (1 mM)

CGTTCtTgTGCAGGAtACAAAgATA (1 mM)

were diluted in MO buffer (5 mg/ml phenol red (Sigma), 4 mM HEPES pH 7.2 (Sigma), $160 \mathrm{mM} \mathrm{KCl} \mathrm{(Sigma))} \mathrm{and} 1.4 \mathrm{nl}$ of MO solution was injected into the yolk of the 1 cell stage embryo. RNA was diluted to $100-300 \mathrm{pg} / \mu \mathrm{l}$ in nuclease free water and $1.4 \mathrm{nl}$ was injected into the cell of the 1 cell stage embryo. Embryos were stage matched, anaesthetised using $0.016 \%$ MESAB in E3 embryo medium and imaged with a Nikon SMZ800 stereo microscope and Canon Eos 1100D camera and software. 
RT-PCR analysis of splicing morpholino injected embryos

Total RNA was extracted using TRIZOL according to manufactures' instructions from 50 pooled $48 \mathrm{hpf}$ AB WT embryos either uninjected or injected with either $1 \mathrm{mM}$ control splice 1 mismatch morpholino, $1 \mathrm{mM}$ flcn splice 1 morpholino, $1 \mathrm{mM}$ control splice 2 mismatch morpholino, $1 \mathrm{mM}$ flcn splice 2 morpholino. Following DNAse treatment using TURBO DNA-free ${ }^{\mathrm{TM}}$ Kit (Ambion AM1907), $1 \mu \mathrm{g}$ total RNA was subjected to RT-PCR using M-MLV Reverse Transcriptase, RNase H Minus, Point Mutant (Promega) and random hexamers (Fermentas). Specific PCR was performed on $5 \mu \mathrm{l}$ cDNA using GoTaq polymerase (Promega) with the following forward 5'TCCCATCACATGACACACAA3' and reverse 5'GAGACTGCGCACACATGC3' primers and the following parameters $94^{\circ} \mathrm{C}$ for $5 \mathrm{~min},\left(94^{\circ} \mathrm{C}\right.$ for $30 \mathrm{secs} \rightarrow 59^{\circ} \mathrm{C}$ for $40 \mathrm{sec} \rightarrow 72^{\circ} \mathrm{C}$ for $30 \mathrm{sec}$ ) $\mathrm{x} 30$ and $72^{\circ} \mathrm{C}$ for $5 \mathrm{~min}$.

\section{Whole-mount in situ hybridization and immunohistochemistry}

Whole-mount in situ hybridization was carried out as described by Thisse and Thisse (62). The flcn gene probe was transcribed directly from cloned cDNA in pCS2+, linearized with NotI (NEB) and transcribed with T7 polymerase (Promega) using Dig labelling mix (Roche). The pcna gene probe (a kind gift from Dr. F. van Eeden (University of Sheffield, United Kingdom)) was linearized with NotI (NEB) and transcribed with SP6 polymerase (Promega) using Dig labelling mix (Roche). Pre-tailbud embryos were fixed in $4 \%$ paraformaldehyde overnight and then manually dechorionated. Embryos older than $24 \mathrm{hpf}$ were manually dechorionated and then fixed in $4 \%$ paraformaldehyde overnight at $4^{\circ} \mathrm{C}$. Embryos were cleared in glycerol and imaged with a Nikon SMZ800 stereo microscope and Canon Eos $1100 \mathrm{D}$ camera.

Double fluorescent whole mount in situ hybridization was carried out as described by Manfroid et al. (63). The flcn and pcna gene probes were labelled with Dig labelling mix (Roche) and DNP-11-UTP (Perkin Elmar), respectively. Probes were detected using either Dig or DNP HRP-conjugated antibodies developed with either Cy3 mono NHS ester (Amersham) or Fluorescein NHS ester (Pierce).

Whole mount immunofluorescence of acetylated alpha tubulin was carried out as follows: embryos were injected with either flcn splice 2 morpholino or mismatch morpholino, stage-matched at $24 \mathrm{hpf}$, manually dechorionated and fixed in $4 \%$ formaldehyde in PBS overnight at $4^{\circ} \mathrm{C}$. Embryos were permeabilised using 10 $\mu \mathrm{g} / \mathrm{ml}$ proteinase $\mathrm{K}$ (Sigma) in PBS containing 0.1\% tween (PBST) for 20 minutes at room temperature, followed by re-fixation for 20 minutes. Non-specific binding sites were blocked by incubation in 10\% newborn calf serum in PBS for 1 hour at room temperature, followed by overnight incubation at $4^{\circ} \mathrm{C}$ in mouse acetylated alpha tublin antibodies (Abcam) diluted 1:200 in block buffer. The following day embryos were washed 4x $30 \mathrm{~min}$ in PBST and re-blocked for 1 hour. Embryos were incubated in sheep-anti-mouse cy3-conjugated antibodies (Sigma) diluted 
1:200 in block buffer overnight at $4^{\circ} \mathrm{C}$, followed by $4 \times 30$ min wash in PBST. Embryos were refixed in $4 \%$ formaldehyde in PBS for 20 minutes at room temperature. Embryos were mounted in Vectashield (Vector labs) and images captured using a Leica LSI TCS Zoom confocal and Leica software. Images were processed using ImageJ software (NIH).

\section{Embryo preparation and confocal imaging}

Tg (wt1b:EGFP) embryos (38) injected with flcn splice 2 morpholino or flcn splice 2 mismatch morpholino were stage matched, anaesthetised and imaged on a Leica TCS LSI confocal. Images were processed using ImageJ.

zFucci embryos (43) were injected with either $1 \mathrm{mM}$ flcn splice2 mismatch morpholino or $1 \mathrm{mM}$ flcn splice 2 morpholino. Embryos were maintained at $28^{\circ} \mathrm{C}$ in Danieaus solution ( $58 \mathrm{mM} \mathrm{NaCl}, 0.7 \mathrm{mM} \mathrm{KCl}, 0.4 \mathrm{mM} \mathrm{MgSO}_{4}, 0.6 \mathrm{mM} \mathrm{Ca}\left(\mathrm{NO}_{3}\right)_{2}, 5.0$ mM HEPES pH 7.6) until bud-stage. A 96-well plate (Greiner Bio-One) was prepared by adding $50 \mu \mathrm{l}$ of $1 \%$ agarose (Bioline) in Danieaus solution covered by $30 \mu \mathrm{l}$ of ethanol (VWR) to each well and inserting a brass 96 pin template into the plate until the agarose set. The template was removed and the plate washed thoroughly with distilled water. Embryos were dechorinated on 1\% agarose in Danieaus solution and transferred with $60 \mu \mathrm{l}$ of Danieaus in to the 96 well agarose plate. Embryos were oriented using a Microlance 3 needle outer diameter $0.51 \mathrm{~mm}$ (BD Biosciences). $40 \mu \mathrm{l}$ of mineral oil (Sigma) was added over the surface of the Danieaus solution. Leica LSI TCS Zoom confocal and software was used to record the time-lapse video. Time-lapse was conducted in xyzt mode using the $532 \mathrm{~nm}$ and $488 \mathrm{~nm}$ lasers. 40 slices were taken for each stack for 11 hours, z volume $358.395 \mu \mathrm{m}$ lens $5 \mathrm{x}$, optical zoom 1.362 step size 9.19, red gain 1200 offset -4 , frame average 4 green gain 1250, offset -5 frame average 8 . For still imaging, 3 slices were taken for each image frame average $3,2 \mathrm{x}$ lens, $\mathrm{z}$ volume $500 \mu \mathrm{m}$.

\section{Statistics - reporter activity}

Analysis of Fucci reporter activity was carried out by statistical evaluation of pixel intensities of fluorescence signals detected for red and green reporters in flcn splice 2 morpholino-injected embryos, compared to mismatch controls. Mean log pixel intensity was calculated for each imaged embryo. This was performed for red and green channels separately. We tested the hypothesis of differential intensity associated with either flcn MO or control MO using a linear mixed-effects modelling framework (64). Morpholino treatment was fitted as a categorical fixed effect and other sources of experimental variation (day, parental fish) were modelled as multilevel random effects. Treatment contrasts were tested using ttests and results were validated by generating parameter confidence distributions using MCMC with $10^{4}$ iterations. 
Statistics - time course analysis

Log-transformed intensity data from embryos injected with flcn mismatch $(n=3)$ or flcn splice 2 morpholino $(\mathrm{n}=4)$ was modelled using Generalised Additive Models, within a mixed-effects modelling framework (GAMM). Cubic smoothing splines were used to model mean trajectories for each morpholino treatment. Following initial visual inspection, biological variation was modelled with separate parameters for each morpholino. A first order autoregressive process was used to model temporal autocorrelation within each time course.

\section{Funding}

Funding for this project was provided by the Myrovlytis Trust to FM and EM and a Dutch Cancer Society (KWF) travel grant to ML.

\section{Acknowledgements}

We would like to thank Dr. Christoph Englert (Fritz Lipmann Institute, Jena, Germany) for the transgenic line $T g(w t 1 b: E G F P)$ and Dr. Atsushi Miyawaki (RIKEN, Japan) for the zFucci lines. 


\section{References}

1. Toro JR, Wei MH, Glenn GM, Weinreich M, Toure O, Vocke C, et al. BHD mutations, clinical and molecular genetic investigations of Birt-Hogg-Dube syndrome: a new series of 50 families and a review of published reports. Journal of medical genetics. 2008 Jun;45(6):321-31. PubMed PMID: 18234728. Pubmed Central PMCID: 2564862.

2. Menko FH, van Steensel MA, Giraud S, Friis-Hansen L, Richard S, Ungari S, et al. BirtHogg-Dube syndrome: diagnosis and management. The lancet oncology. 2009 Dec;10(12):1199-206. PubMed PMID: 19959076.

3. Schmidt LS, Nickerson ML, Warren MB, Glenn GM, Toro JR, Merino MJ, et al. Germline BHD-mutation spectrum and phenotype analysis of a large cohort of families with BirtHogg-Dube syndrome. American journal of human genetics. 2005 Jun;76(6):1023-33. PubMed PMID: 15852235. Pubmed Central PMCID: 1196440.

4. Nickerson ML, Warren MB, Toro JR, Matrosova V, Glenn G, Turner ML, et al. Mutations in a novel gene lead to kidney tumors, lung wall defects, and benign tumors of the hair follicle in patients with the Birt-Hogg-Dube syndrome. Cancer cell. 2002 Aug;2(2):15764. PubMed PMID: 12204536.

5. Wei MH, Blake PW, Shevchenko J, Toro JR. The folliculin mutation database: an online database of mutations associated with Birt-Hogg-Dube syndrome. Human mutation. 2009 Sep;30(9):E880-90. PubMed PMID: 19562744. Pubmed Central PMCID: 3234166.

6. Lim DH, Rehal PK, Nahorski MS, Macdonald F, Claessens T, Van Geel M, et al. A new locus-specific database (LSDB) for mutations in the folliculin (FLCN) gene. Human mutation. 2010 Jan;31(1):E1043-51. PubMed PMID: 19802896.

7. Baba M, Hong SB, Sharma N, Warren MB, Nickerson ML, Iwamatsu A, et al. Folliculin encoded by the BHD gene interacts with a binding protein, FNIP1, and AMPK, and is involved in AMPK and mTOR signaling. Proceedings of the National Academy of Sciences of the United States of America. 2006 Oct 17;103(42):15552-7. PubMed PMID: 17028174. Pubmed Central PMCID: 1592464.

8. Chen J, Futami K, Petillo D, Peng J, Wang P, Knol J, et al. Deficiency of FLCN in mouse kidney led to development of polycystic kidneys and renal neoplasia. PloS one. 2008;3(10):e3581. PubMed PMID: 18974783. Pubmed Central PMCID: 2570491.

9. Hartman TR, Nicolas E, Klein-Szanto A, Al-Saleem T, Cash TP, Simon MC, et al. The role of the Birt-Hogg-Dube protein in mTOR activation and renal tumorigenesis. Oncogene. 2009 Apr 2;28(13):1594-604. PubMed PMID: 19234517. Pubmed Central PMCID: 2664853.

10. Hudon V, Sabourin S, Dydensborg AB, Kottis V, Ghazi A, Paquet M, et al. Renal tumour suppressor function of the Birt-Hogg-Dube syndrome gene product folliculin. Journal of medical genetics. 2010 Mar;47(3):182-9. PubMed PMID: 19843504.

11. Preston RS, Philp A, Claessens T, Gijezen L, Dydensborg AB, Dunlop EA, et al. Absence of the Birt-Hogg-Dube gene product is associated with increased hypoxia-inducible factor transcriptional activity and a loss of metabolic flexibility. Oncogene. $2011 \mathrm{Mar}$ 10;30(10):1159-73. PubMed PMID: 21057536. Pubmed Central PMCID: 3787473.

12. Hong SB, Oh H, Valera VA, Baba M, Schmidt LS, Linehan WM. Inactivation of the FLCN tumor suppressor gene induces TFE3 transcriptional activity by increasing its nuclear localization. PloS one. 2010;5(12):e15793. PubMed PMID: 21209915. Pubmed Central PMCID: 3012117.

13. Betschinger J, Nichols J, Dietmann S, Corrin PD, Paddison PJ, Smith A. Exit from pluripotency is gated by intracellular redistribution of the bHLH transcription factor Tfe3. Cell. 2013 Apr 11;153(2):335-47. PubMed PMID: 23582324. Pubmed Central PMCID: 3661979. 
14. Luijten MN, Basten SG, Claessens T, Vernooij M, Scott CL, Janssen R, et al. Birt-HoggDube syndrome is a novel ciliopathy. Human molecular genetics. 2013 Nov 1;22(21):4383-97. PubMed PMID: 23784378. Pubmed Central PMCID: 3792695.

15. Petit CS, Roczniak-Ferguson A, Ferguson SM. Recruitment of folliculin to lysosomes supports the amino acid-dependent activation of Rag GTPases. The Journal of cell biology. 2013 Sep 30;202(7):1107-22. PubMed PMID: 24081491. Pubmed Central PMCID: 3787382.

16. Tsun ZY, Bar-Peled L, Chantranupong L, Zoncu R, Wang T, Kim C, et al. The folliculin tumor suppressor is a GAP for the RagC/D GTPases that signal amino acid levels to mTORC1. Molecular cell. 2013 Nov 21;52(4):495-505. PubMed PMID: 24095279. Pubmed Central PMCID: 3867817.

17. Possik E, Jalali Z, Nouet Y, Yan M, Gingras MC, Schmeisser K, et al. Folliculin regulates ampk-dependent autophagy and metabolic stress survival. PLoS genetics. 2014 Apr;10(4):e1004273. PubMed PMID: 24763318.

18. Yan M, Gingras MC, Dunlop EA, Nouet Y, Dupuy F, Jalali Z, et al. The tumor suppressor folliculin regulates AMPK-dependent metabolic transformation. The Journal of clinical investigation. 2014 Apr 24. PubMed PMID: 24762438.

19. Singh SR, Zhen W, Zheng Z, Wang H, Oh SW, Liu W, et al. The Drosophila homolog of the human tumor suppressor gene BHD interacts with the JAK-STAT and Dpp signaling pathways in regulating male germline stem cell maintenance. Oncogene. 2006 Sep 28;25(44):5933-41. PubMed PMID: 16636660.

20. Nahorski MS, Seabra L, Straatman-Iwanowska A, Wingenfeld A, Reiman A, Lu X, et al. Folliculin interacts with p0071 (plakophilin-4) and deficiency is associated with disordered RhoA signalling, epithelial polarization and cytokinesis. Human molecular genetics. 2012 Dec 15;21(24):5268-79. PubMed PMID: 22965878. Pubmed Central PMCID: 3755511.

21. Medvetz DA, Khabibullin D, Hariharan V, Ongusaha PP, Goncharova EA, Schlechter T, et al. Folliculin, the product of the Birt-Hogg-Dube tumor suppressor gene, interacts with the adherens junction protein p0071 to regulate cell-cell adhesion. PloS one. 2012;7(11):e47842. PubMed PMID: 23139756. Pubmed Central PMCID: 3490959.

22. Bastola P, Stratton Y, Kellner E, Mikhaylova O, Yi Y, Sartor MA, et al. Folliculin contributes to VHL tumor suppressing activity in renal cancer through regulation of autophagy. PloS one. 2013;8(7):e70030. PubMed PMID: 23922894. Pubmed Central PMCID: 3726479.

23. Dunlop EA, Seifan S, Claessens T, Behrends C, Kamps M, Rozycka E, et al. FLCN, a novel autophagy component, interacts with GABARAP and is regulated by ULK1 phosphorylation. Autophagy. 2014;0:0 - -1.

24. Hasumi Y, Baba M, Ajima R, Hasumi H, Valera VA, Klein ME, et al. Homozygous loss of BHD causes early embryonic lethality and kidney tumor development with activation of mTORC1 and mTORC2. Proceedings of the National Academy of Sciences of the United States of America. 2009 Nov 3;106(44):18722-7. PubMed PMID: 19850877. Pubmed Central PMCID: 2765925.

25. Baba M, Furihata M, Hong SB, Tessarollo L, Haines DC, Southon E, et al. Kidneytargeted Birt-Hogg-Dube gene inactivation in a mouse model: Erk1/2 and Akt-mTOR activation, cell hyperproliferation, and polycystic kidneys. Journal of the National Cancer Institute. 2008 Jan 16;100(2):140-54. PubMed PMID: 18182616. Pubmed Central PMCID: 2704336.

26. Cash TP, Gruber JJ, Hartman TR, Henske EP, Simon MC. Loss of the Birt-Hogg-Dube tumor suppressor results in apoptotic resistance due to aberrant TGFbeta-mediated transcription. Oncogene. 2011 Jun 2;30(22):2534-46. PubMed PMID: 21258407. Pubmed Central PMCID: 3109270. 
27. Okimoto K, Sakurai J, Kobayashi T, Mitani H, Hirayama Y, Nickerson ML, et al. A germline insertion in the Birt-Hogg-Dube (BHD) gene gives rise to the Nihon rat model of inherited renal cancer. Proceedings of the National Academy of Sciences of the United States of America. 2004 Feb 17;101(7):2023-7. PubMed PMID: 14769940. Pubmed Central PMCID: 357045.

28. Lingaas F, Comstock KE, Kirkness EF, Sorensen A, Aarskaug T, Hitte C, et al. A mutation in the canine BHD gene is associated with hereditary multifocal renal cystadenocarcinoma and nodular dermatofibrosis in the German Shepherd dog. Human molecular genetics. 2003 Dec 1;12(23):3043-53. PubMed PMID: 14532326.

29. Amatruda JF, Shepard JL, Stern HM, Zon LI. Zebrafish as a cancer model system. Cancer cell. 2002 Apr;1(3):229-31. PubMed PMID: 12086858.

30. Lam SH, Wu YL, Vega VB, Miller LD, Spitsbergen J, Tong Y, et al. Conservation of gene expression signatures between zebrafish and human liver tumors and tumor progression. Nature biotechnology. 2006 Jan;24(1):73-5. PubMed PMID: 16327811.

31. Langenau DM, Traver D, Ferrando AA, Kutok JL, Aster JC, Kanki JP, et al. Myc-induced T cell leukemia in transgenic zebrafish. Science. 2003 Feb 7;299(5608):887-90. PubMed PMID: 12574629.

32. Wodarz A, Nathke I. Cell polarity in development and cancer. Nature cell biology. 2007 Sep;9(9):1016-24. PubMed PMID: 17762893.

33. Nepal C, Hadzhiev Y, Previti C, Haberle V, Li N, Takahashi H, et al. Dynamic regulation of the transcription initiation landscape at single nucleotide resolution during vertebrate embryogenesis. Genome research. 2013 Nov;23(11):1938-50. PubMed PMID: 24002785. Pubmed Central PMCID: 3814893.

34. Sievers F, Wilm A, Dineen D, Gibson TJ, Karplus K, Li W, et al. Fast, scalable generation of high-quality protein multiple sequence alignments using Clustal Omega. Molecular systems biology. 2011;7:539. PubMed PMID: 21988835. Pubmed Central PMCID: 3261699.

35. Goujon M, McWilliam H, Li W, Valentin F, Squizzato S, Paern J, et al. A new bioinformatics analysis tools framework at EMBL-EBI. Nucleic acids research. 2010 Jul;38(Web Server issue):W695-9. PubMed PMID: 20439314. Pubmed Central PMCID: 2896090.

36. Waterhouse AM, Procter JB, Martin DM, Clamp M, Barton GJ. Jalview Version 2--a multiple sequence alignment editor and analysis workbench. Bioinformatics. 2009 May 1;25(9):1189-91. PubMed PMID: 19151095. Pubmed Central PMCID: 2672624.

37. Ferrante MI, Romio L, Castro S, Collins JE, Goulding DA, Stemple DL, et al. Convergent extension movements and ciliary function are mediated by ofd1, a zebrafish orthologue of the human oral-facial-digital type 1 syndrome gene. Human molecular genetics. 2009 Jan 15;18(2):289-303. PubMed PMID: 18971206. Pubmed Central PMCID: 2638777.

38. Perner B, Englert C, Bollig F. The Wilms tumor genes wt1a and wt1b control different steps during formation of the zebrafish pronephros. Developmental biology. $2007 \mathrm{Sep}$ 1;309(1):87-96. PubMed PMID: 17651719.

39. Pathak N, Obara T, Mangos S, Liu Y, Drummond IA. The zebrafish fleer gene encodes an essential regulator of cilia tubulin polyglutamylation. Molecular biology of the cell. 2007 Nov;18(11):4353-64. PubMed PMID: 17761526. Pubmed Central PMCID: 2043541.

40. Kramer-Zucker AG, Olale F, Haycraft CJ, Yoder BK, Schier AF, Drummond IA. Ciliadriven fluid flow in the zebrafish pronephros, brain and Kupffer's vesicle is required for normal organogenesis. Development. 2005 Apr;132(8):1907-21. PubMed PMID: 15790966. 
41. Kawai A, Kobayashi T, Hino O. Folliculin regulates cyclin D1 expression through cisacting elements in the 3' untranslated region of cyclin D1 mRNA. International journal of oncology. 2013 May;42(5):1597-604. PubMed PMID: 23525507.

42. Laviolette LA, Wilson J, Koller J, Neil C, Hulick P, Rejtar T, et al. Human folliculin delays cell cycle progression through late S and G2/M-phases: effect of phosphorylation and tumor associated mutations. PloS one. 2013;8(7):e66775. PubMed PMID: 23874397. Pubmed Central PMCID: 3708955.

43. Sugiyama M, Sakaue-Sawano A, Iimura T, Fukami K, Kitaguchi T, Kawakami K, et al. Illuminating cell-cycle progression in the developing zebrafish embryo. Proceedings of the National Academy of Sciences of the United States of America. 2009 Dec 8;106(49):20812-7. PubMed PMID: 19923430. Pubmed Central PMCID: 2779202.

44. Valk E, Loog M. Multiple Pho85-dependent mechanisms control G1 cyclin abundance in response to nutrient stress. Molecular and cellular biology. 2013 Apr;33(7):1270-2. PubMed PMID: 23382071. Pubmed Central PMCID: 3624275.

45. Liu W, Chen Z, Ma Y, Wu X, Jin Y, Hou S. Genetic characterization of the Drosophila birthogg-dube syndrome gene. PloS one. 2013;8(6):e65869. PubMed PMID: 23799055. Pubmed Central PMCID: 3684598.

46. Landsverk ML, Weiser DC, Hannibal MC, Kimelman D. Alternative splicing of sept9a and sept9b in zebrafish produces multiple mRNA transcripts expressed throughout development. PloS one. 2010;5(5):e10712. PubMed PMID: 20502708. Pubmed Central PMCID: 2873287.

47. Lumsden AL, Henshall TL, Dayan S, Lardelli MT, Richards RI. Huntingtin-deficient zebrafish exhibit defects in iron utilization and development. Human molecular genetics. 2007 Aug 15;16(16):1905-20. PubMed PMID: 17567778.

48. Raldua D, Andre M, Babin PJ. Clofibrate and gemfibrozil induce an embryonic malabsorption syndrome in zebrafish. Toxicology and applied pharmacology. 2008 May 1;228(3):301-14. PubMed PMID: 18358510.

49. Leskow FC, Holloway BA, Wang H, Mullins MC, Kazanietz MG. The zebrafish homologue of mammalian chimerin Rac-GAPs is implicated in epiboly progression during development. Proc Natl Acad Sci U S A. 2006 Apr 4;103(14):5373-8. PubMed PMID: 16569702. Pubmed Central PMCID: 1459362.

50. Cheng JC, Miller AL, Webb SE. Organization and function of microfilaments during late epiboly in zebrafish embryos. Developmental dynamics : an official publication of the American Association of Anatomists. 2004 Oct;231(2):313-23. PubMed PMID: 15366008.

51. Niehrs C, Acebron SP. Mitotic and mitogenic Wnt signalling. The EMBO journal. 2012 Jun 13;31(12):2705-13. PubMed PMID: 22617425. Pubmed Central PMCID: 3380213.

52. Heisenberg CP, Tada M, Rauch GJ, Saude L, Concha ML, Geisler R, et al. Silberblick/Wnt11 mediates convergent extension movements during zebrafish gastrulation. Nature. 2000 May 4;405(6782):76-81. PubMed PMID: 10811221.

53. Blader P, Strahle U. Casting an eye over cyclopia. Nature. 1998 Sep 10;395(6698):1123. PubMed PMID: 9744263.

54. Hayes M, Naito M, Daulat A, Angers S, Ciruna B. Ptk7 promotes non-canonical Wnt/PCP-mediated morphogenesis and inhibits Wnt/beta-catenin-dependent cell fate decisions during vertebrate development. Development. 2013 Apr;140(8):1807-18. PubMed PMID: 23533179.

55. Yuan Y, Niu CC, Deng G, Li ZQ, Pan J, Zhao C, et al. The Wnt5a/Ror2 noncanonical signaling pathway inhibits canonical Wnt signaling in K562 cells. International journal of molecular medicine. 2011 Jan;27(1):63-9. PubMed PMID: 21069266.

56. Jinek M, Chylinski K, Fonfara I, Hauer M, Doudna JA, Charpentier E. A programmable dual-RNA-guided DNA endonuclease in adaptive bacterial immunity. Science. 2012 Aug 17;337(6096):816-21. PubMed PMID: 22745249. 
57. Hwang WY, Fu Y, Reyon D, Maeder ML, Kaini P, Sander JD, et al. Heritable and precise zebrafish genome editing using a CRISPR-Cas system. PloS one. 2013;8(7):e68708. PubMed PMID: 23874735. Pubmed Central PMCID: 3706373.

58. Jao LE, Wente SR, Chen W. Efficient multiplex biallelic zebrafish genome editing using a CRISPR nuclease system. Proceedings of the National Academy of Sciences of the United States of America. 2013 Aug 20;110(34):13904-9. PubMed PMID: 23918387. Pubmed Central PMCID: 3752207.

59. Auer TO, Duroure K, De Cian A, Concordet JP, Del Bene F. Highly efficient CRISPR/Cas9-mediated knock-in in zebrafish by homology-independent DNA repair. Genome research. 2014 Jan;24(1):142-53. PubMed PMID: 24179142. Pubmed Central PMCID: 3875856.

60. Kimmel CB, Ballard WW, Kimmel SR, Ullmann B, Schilling TF. Stages of embryonic development of the zebrafish. Developmental dynamics : an official publication of the American Association of Anatomists. 1995 Jul;203(3):253-310. PubMed PMID: 8589427.

61. Westerfield M. The Zebrafish Book. A Guide for the Laboratory Use of Zebrafish (Danio rerio). 4th ed. Eugene: University of Oregon Press; 2000.

62. Thisse $\mathrm{C}$, Thisse B. High-resolution in situ hybridization to whole-mount zebrafish embryos. Nature protocols. 2008;3(1):59-69. PubMed PMID: 18193022.

63. Manfroid I, Delporte F, Baudhuin A, Motte P, Neumann CJ, Voz ML, et al. Reciprocal endoderm-mesoderm interactions mediated by fgf24 and fgf10 govern pancreas development. Development. 2007 Nov;134(22):4011-21. PubMed PMID: 17942484.

64. Pinheiro JC, Bates DM. Mixed-effects models in S and S-PLUS: New York ; [London] : Springer, c2000 (2009 printing). 



\section{CHAPTER 4}

\section{Familial multiple discoid fibromas, a Birt-Hogg-Dubé syndrome lookalike, is caused by a truncating FNIP1 mutation}

To be submitted

Monique N.H. Luijten, Theo M. Starink, Sara Seifan, Emma Kenyon, Stephen Land, Jennifer A. Easton, Arjan C. Houweling, Martijn B. van Doorn, Edward M. Leter, Elisabeth H. Jaspars, Paul C. Johannesma, Eric F.A.M. Hennekam , Johan J.P. Gille, Ferenc Müller, Barry J. Coull, Andrew Tee, Fred H. Menko, Maurice A.M. van Steensel, Quinten Waisfisz 


\section{Abstract}

We recently delineated familial multiple discoid fibromas (FMDF), an autosomal dominant disorder that mimics the cutaneous manifestations of the cancer-associated Birt-Hogg-Dubé syndrome (BHD). We showed that it is not associated with the germline FLCN (folliculin) mutations causative of BHD. Here we demonstrate that FMDF is caused by a heterozygous truncating germline mutation (c.1989delT) in the FNIP1 gene, encoding the highly conserved folliculin interacting protein 1 (FNIP1), whose function is largely unknown. The mutation causes nonsense-mediated mRNA decay, suggesting haploinsufficiency as the disease mechanism. Similar to FLCN, FNIP1 localizes to the primary cilium and centrosome in various cell types. However, in vitro and in vivo studies revealed no ciliary defects following FNIP1 knockdown, suggesting that FNIP1, unlike FLCN, is not required for ciliogenesis. Using a novel zebrafish model, we demonstrate that Fnip1 is expressed in a similar pattern to Flcn during development and is required for normal development. FMDF patients appear to not have any systemic phenotype, in contrast to patients with BHD. Our observations indicate that, while FLCN and FNIP1 interact and exhibit similar intracellular localization, FNIP1 might be dispensable for most of FLCN's functions. 


\section{Report}

Recently, we studied a series of families with familial multiple trichodiscomas (FMDF; OMIM \%190340), in which patients develop skin lesions resembling those found in Birt-Hogg-Dubé syndrome (BHD; OMIM \#135150, Figure S1A and B). BHD is an autosomal dominant disorder, characterized by benign skin tumors called fibrofolliculomas, lung cysts, pneumothorax and renal cell cancer. It is caused by germline mutations in the gene encoding folliculin (FLCN) (1-3). The BHD fibrofolliculomas are cystic tumors consisting of epithelial strands (Figure S1D) that appear after age 25. Familial trichodiscomas have a very similar clinical presentation but have three distinguishing features: they consist mostly of fibrous stroma (Figure S1C and (4)), are of childhood onset and are often localized on the ears. Recently, we confirmed that trichodiscoma development is a distinct syndrome not linked to the FLCN locus (5). We found no evidence for pulmonary or renal complications and renamed the condition familial multiple discoid fibromas (FMDF), in order to avoid any confusion with BHD (5).
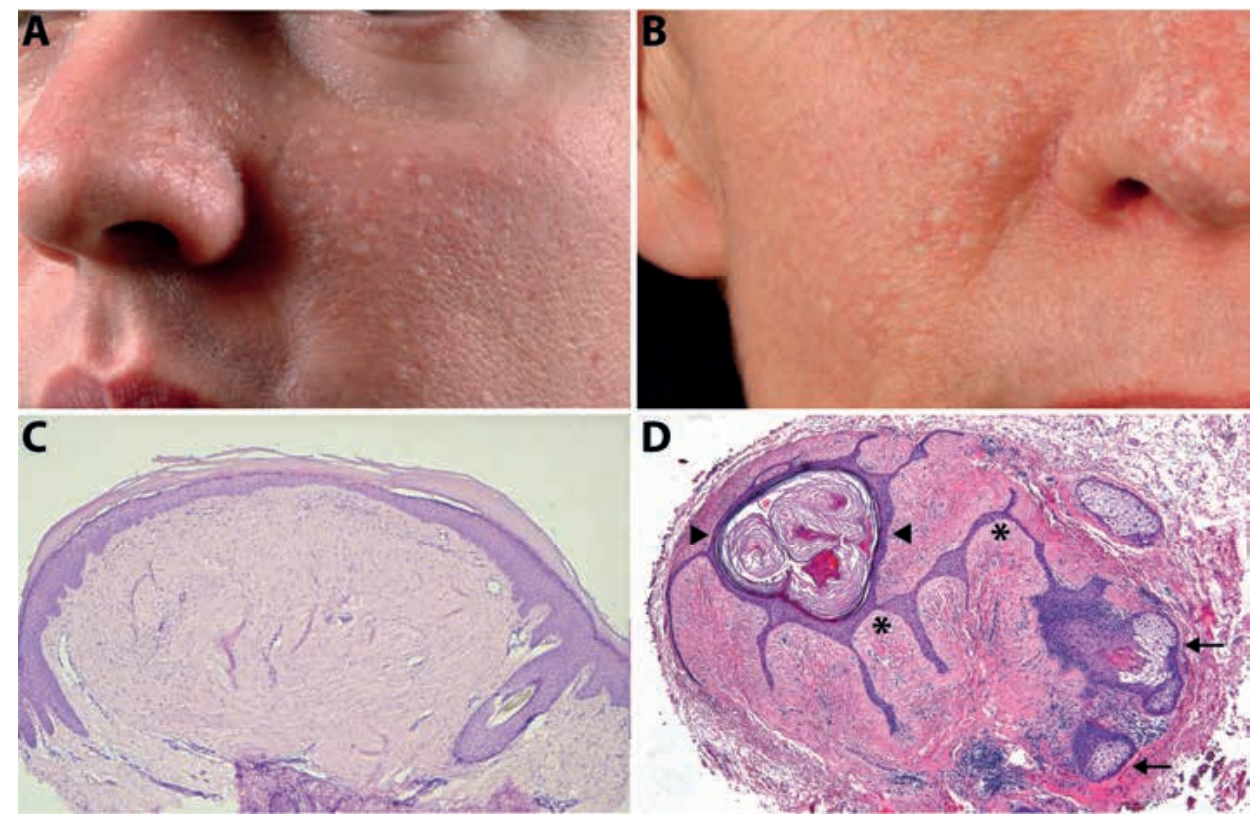

Supplement Figure S1 - Discoid fibromas and BHD fibrofolliculomas are have a similar clinical phenotype but are histologically distinct

(A) The FMDF phenotype. (B) The BHD skin phenotype. Reprinted from (6). (C) Histopathology of a discoid fibroma, which consists mainly of fibrous stroma, suggesting that it is a connective tissue tumour. (D) Histopathology (composite of 4 images) of a fibrofolliculoma. There is a clear cyst containing keratinaceous material (arrowheads) and pronounced sebaceous differentiation (arrows). Thick epithelial strands connect the sebaceous glands to the central cystic mass (asterisk). H\&E; reprinted by permission from Macmillan Publishers Ltd: Journal of Investigative Dermatology (7) copyright 2007. 


\section{CHAPTER 4}

In 2006, a novel binding partner for FLCN was identified, folliculin interacting protein 1 (FNIP1) (8). FNIP1 interacts with and is phosphorylated by AMPK and its overexpression facilitates FLCN phosphorylation, suggesting that FNIP1 has a role in cellular energy and/or nutrient sensing through AMPK (8). In 2008, two groups identified a second novel binding partner for FLCN, FNIP1-like or Folliculin interacting protein 2 (FNIPL/FNIP2/MAP01), a FNIP1 paralog (9, 10), which possibly has a role in AMPK-mediated apoptosis triggered by DNA alkylation (11). As the clinical presentation of the BHD fibrofolliculomas and the discoid fibromas is similar, we hypothesized that FMDF could be caused by germline mutations in one of these folliculin interacting proteins.

Accordingly, we found co-segregation of FMDF with the FNIP1 locus in nine Dutch families, while excluding FNIP2. In these families, all available FMDF cases (n = 27) carried a heterozygous germline mutation in FNIP1 (c.1989delT), which was not present in any of the unaffected family members (Figure 1B). Eight families originate from the same Dutch village, which is a former island and forms a small ethnic isolate. Genealogical studies confirmed common ancestry of these families, suggesting a founder effect (Figure 1A). Clinical re-evaluation of affected adult family members with a specific focus on lungs and kidneys again did not reveal any systemic abnormalities (data not shown).

The c.1989delT FNIP1 mutation predicts the frameshift p.Val663fsX6, which is a conserved residue in vertebrates (Figure 1D). To determine if FNIP1-Val663fsX6 is expressed or broken down by nonsense-mediated mRNA decay, fibroblasts were obtained from discoid fibroma biopsy material. Genomic sequencing of the FNIP1 locus confirmed presence of heterozygous c.1989delT; no somatic second hits were detected (data not shown). Sequencing of cDNA from these fibroblasts showed minimal presence of mutant mRNA, which increased after incubation with puromycin (an inhibitor of nonsense-mediated mRNA decay) (Figure 1C). In addition, no wild-type or Val663fsX6 FNIP1 was detected by Western blot in these cells (Figure 1E). Together, these data indicate that FMDF is most likely caused by FNIP1 haplo-insufficiency.

Although we did not detect FNIP1-Val663fsX6 by Western blot in tumor fibroblasts, minimal amounts of the truncated protein could still be present within the cells, below the detection level of the Western blot, interfering with the function of the wild-type protein. FNIP1 has been reported to interact with FLCN and with FNIP2 $(8,10)$. Deletion of the $237 \mathrm{C}$-terminal amino acids of FNIP1 was shown to result in decreased FLCN binding (8). Accordingly, using coimmunoprecipitation we found impaired binding of FNIP1-Val663fsX6 to both endogenous (Figure S2A) and overexpressed FLCN (Figure S2B). In keeping with previous reports showing that deletion of all five conserved domains of FNIP1 is necessary to abolish FNIP2 binding (10), we found that both wild-type and mutant FNIP1 interact with FNIP2 in co-immunoprecipitation experiments (Figure S2C). 


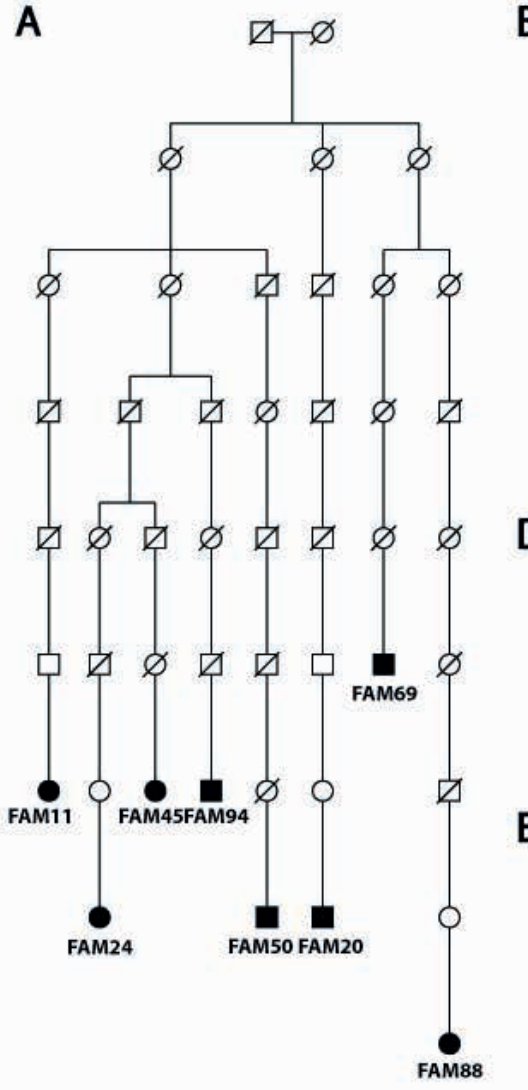

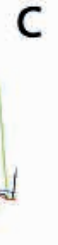

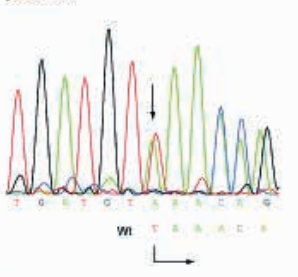

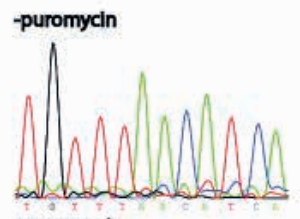

+puromycin

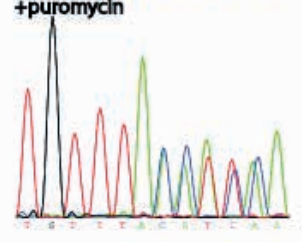

m

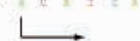

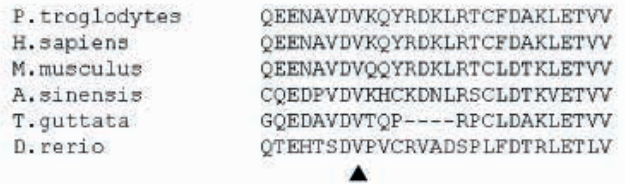

E

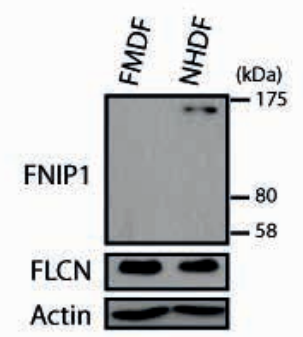

Figure 1 - FMDF is caused by a mutation in FNIP1

(A) Pedigree showing the relation between eight identified FMDF kindreds derived from a Dutch genetic isolate. (B) Electropherogram showing the heterozygous germline FNIP1 mutation c.1989delT in genomic DNA of an FMDF patient (bottom panel) versus the wild-type sequence of an unaffected family member (top panel). (C) FNIP1-c.1989delT mRNA is degraded by nonsense-mediated decay. Electropherogram (antisense orientation) of cDNA isolated from discoid fibroma primary fibroblasts incubated with (bottom panel) or without (top panel) $200 \mu \mathrm{g} / \mathrm{ml}$ puromycin for six hours (to inhibit nonsense-mediated decay). (D) Partial alignment of FNIP1 orthologs in vertebrates including residue 663 (arrowhead), showing conservation. (E) Western blot on whole cell extract from FMDF patient fibroblasts, showing decreased expression of full-length FNIP1 (128 kDa) compared to NHDF $\alpha$ control and no detection of FNIP1-Val663fsX6 (70 kDa). No difference in FLCN level was detected. Actin was used as a loading control. 
CHAPTER 4

A

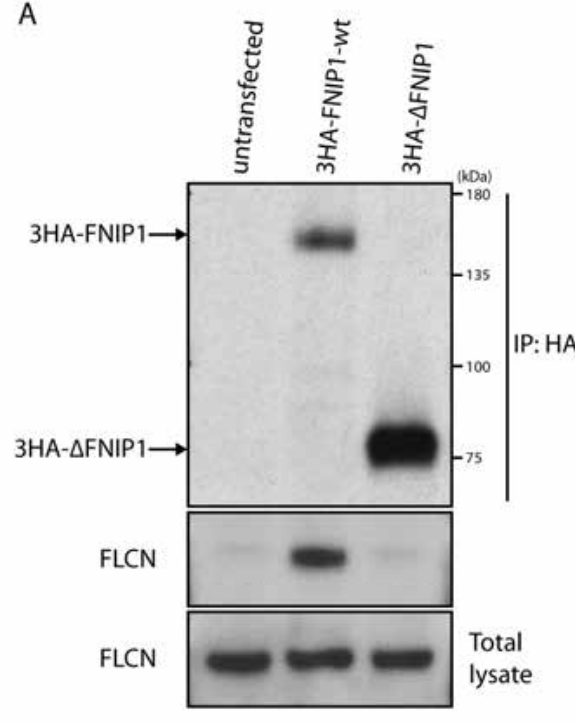

B
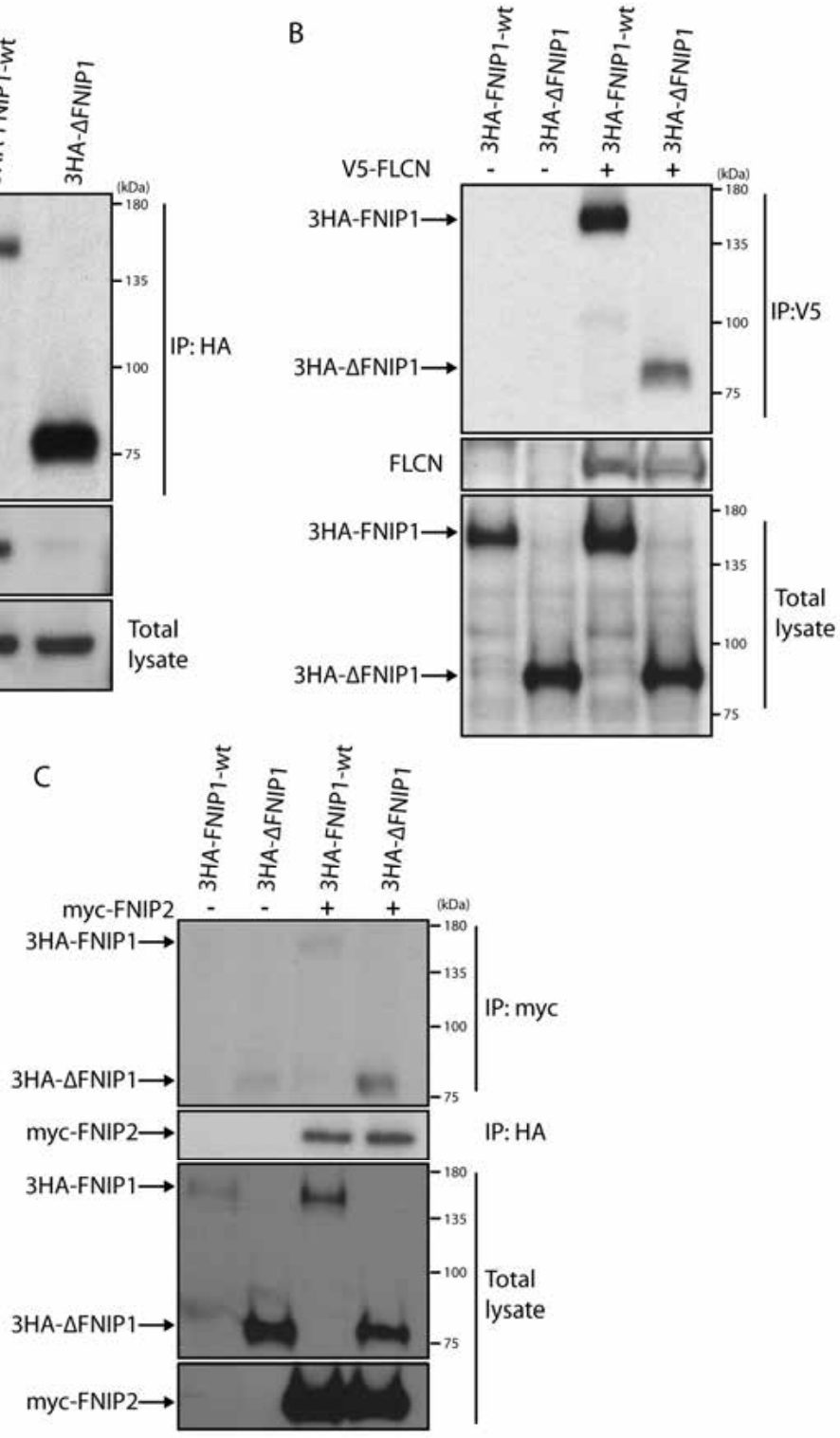

Supplement Figure S2 - FNIP1-Val663fsX6 shows impaired binding to FLCN but not to FNIP2

(A) HA immunoprecipitation on extract of HEK293 cells transiently transfected with indicated 3HAFNIP1 constructs, showing decreased binding of endogenous FLCN to FNIP1-Val663fsX6 ( $\triangle$ FNIP1) compared to FNIP1 wild-type. (B) V5 immunoprecipitation on extract of HEK293 cells transiently transfected with indicated 3HA-FNIP1 constructs with or without V5-FLCN-wt, showing decreased binding of $\triangle$ FNIP1 compared to FNIP1 wild-type. (C) Myc (top panel) or HA (second panel) immunoprecipitation on extract of HEK293 cells transiently transfected with indicated 3HA-FNIP1 constructs with or without myc-FNIP2-wt, showing binding of FNIP1 wild-type and $\triangle$ FNIP1 to FNIP2. 


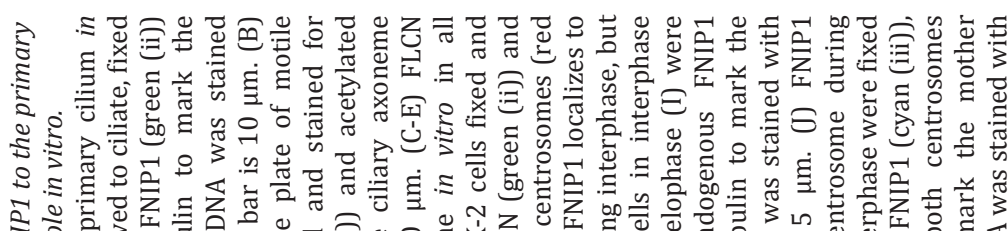

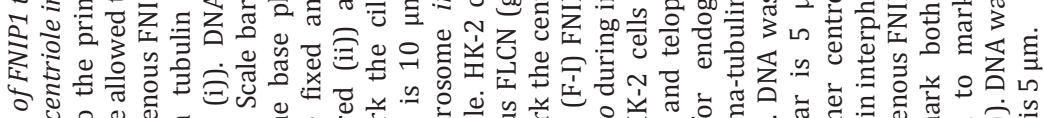

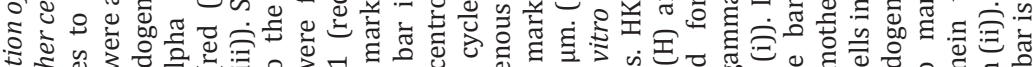

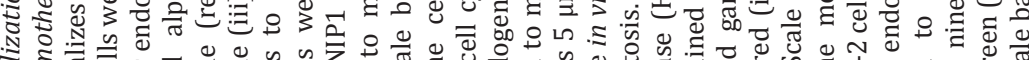

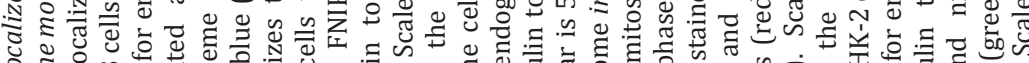

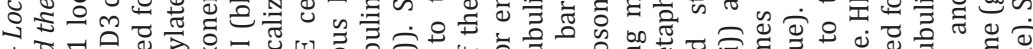

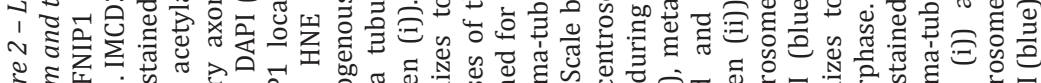

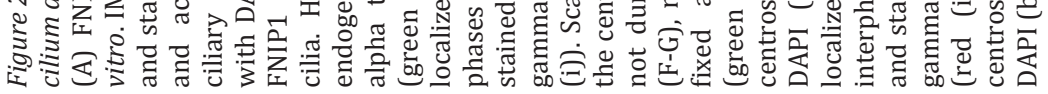

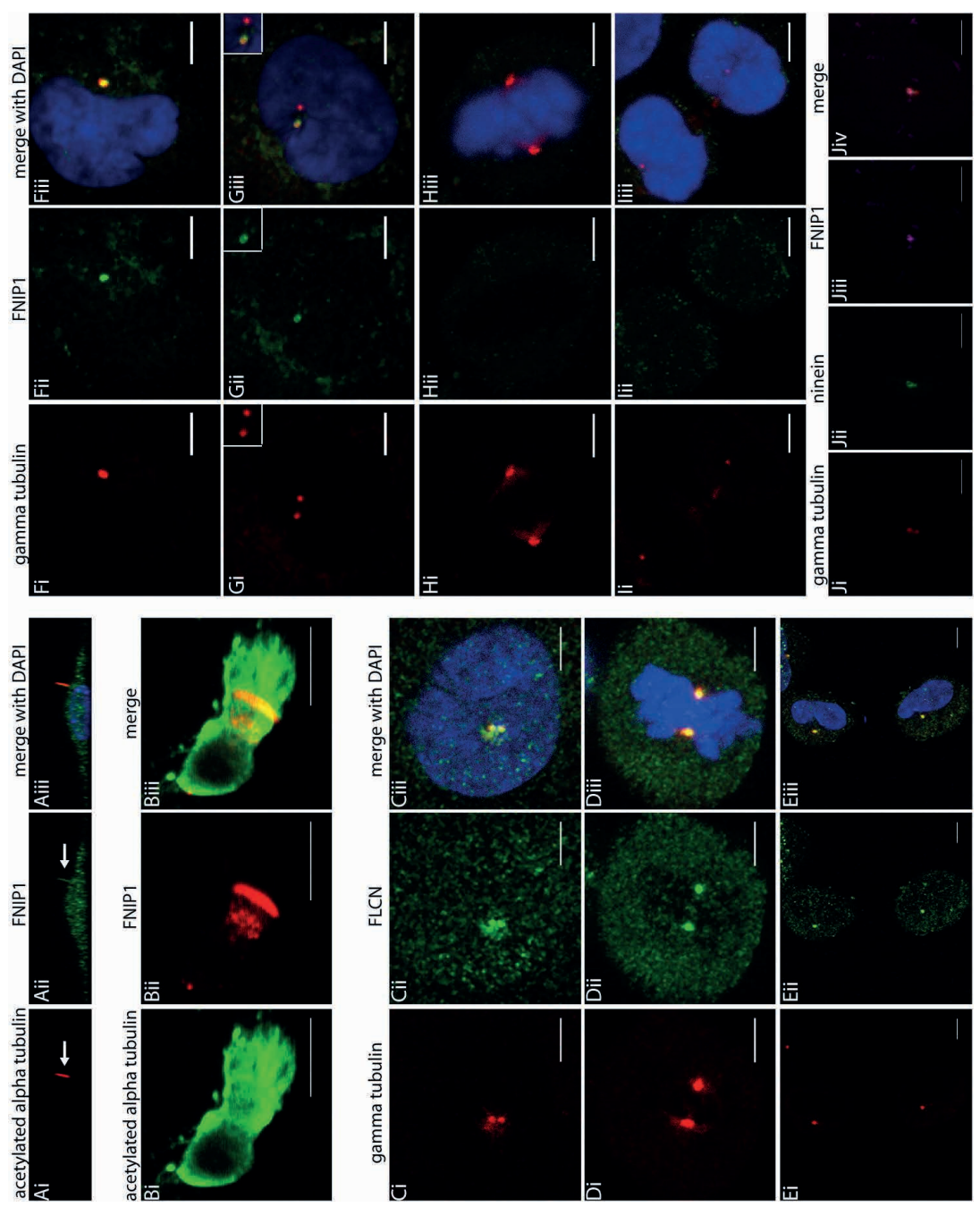


$৩$
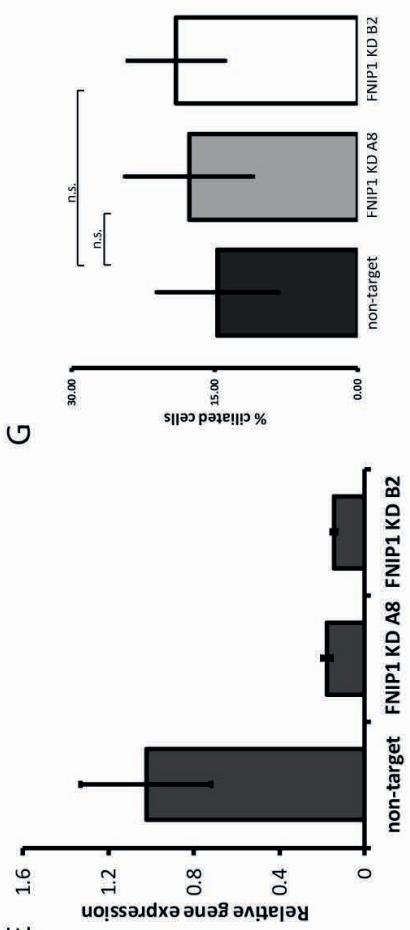

山

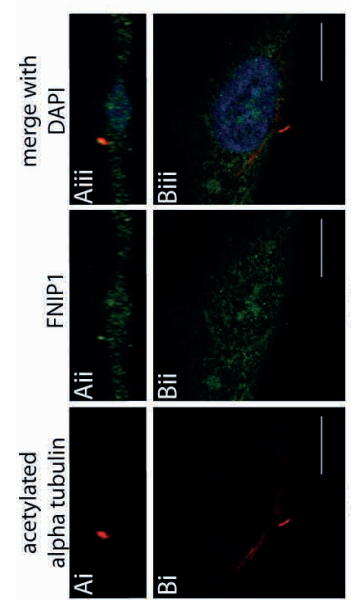

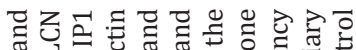

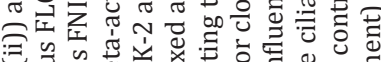

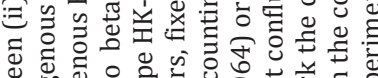

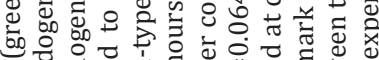

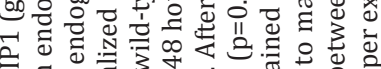

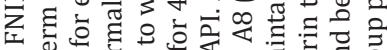

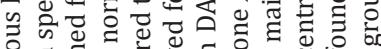

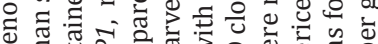

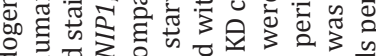

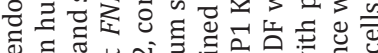
๘

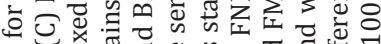

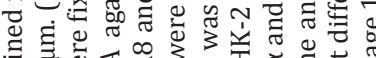

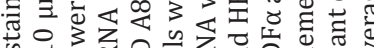

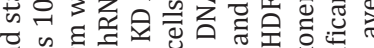

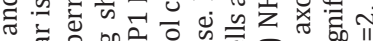
ठ कै के

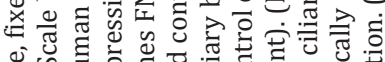

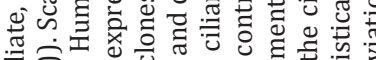

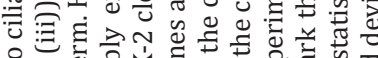

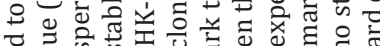

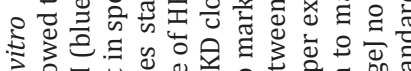

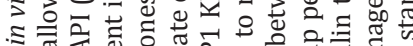

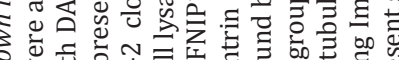
वे चี

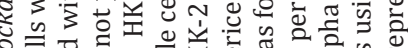

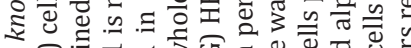

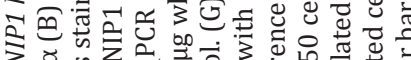

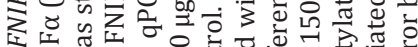

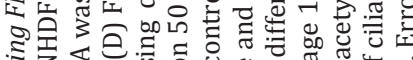

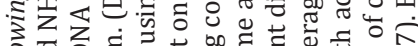

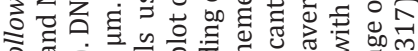
응워

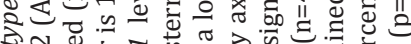

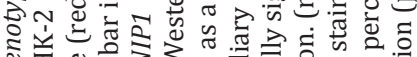

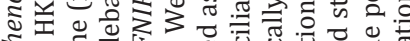
₹ 0 ह चٓ

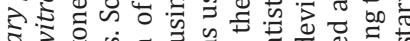

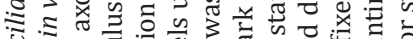

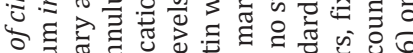

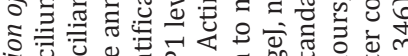
Uण

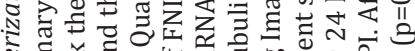
क्ष

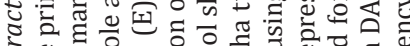

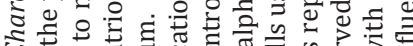

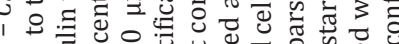
ก ผ 정

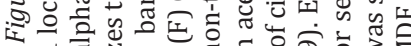

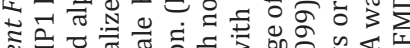

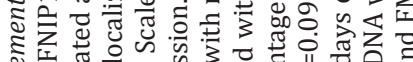

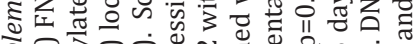

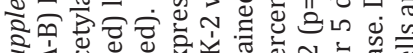

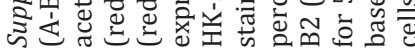


As we have previously shown that FLCN localizes to primary cilia in vitro (12), we aimed to determine FNIP1's intracellular localization. Similar to FLCN, FNIP1 clearly and specifically localizes to the cytoplasm and primary cilia in various cell lines (Figure 2A and Figure S3A and B) and to the base plate of motile cilia (Figure 2B). A ciliary role for FNIP1 would be consistent with our recent finding that BHD is a novel ciliopathy (12), a disease caused by malfunctioning of the primary cilium (13). To test whether FNIP1 has a role in ciliogenesis, we generated a stable knockdown of FNIP1 in HK-2 cells using shRNA. After verification of the knockdown by qPCR (Figure S3E) and Western blot (Figure S3F) the cells showed no gross defects in morphology or growth rate (data not shown). No difference in the percentage of ciliated cells was found in FNIP1 knockdown cells when compared to the non-target control after 48 hours of serum starvation (Figure S3G). As shown in Figure S3H, ciliation was also not affected in the tumor fibroblasts derived from a discoid fibroma when compared to NHDF $\alpha$ control fibroblasts.

In contrast to FLCN, which localizes to the centriole and annulus in human spermatozoa (Figure S3C and (12)), we could not detect any specific staining for FNIP1 in these cells (Figure S3D). Furthermore, FLCN has been shown to localize to the centrosome during all stages of the cell cycle (Figure 2C-E and (12)). FNIP1 also localizes to the centrosome (Figure 2F). However, after duplication FNIP1 only localizes to one of the centrosomes where it seems to form two distinct spots around the tubulin core (Figure 2G). It is displaced from this centrosome during mitosis (Figure $2 \mathrm{H}$ and I). To determine if this was the mother or the daughter centrosome we stained the cells for ninein, a component of the subdistal appendages which are only present on the mother centriole (14). As shown in Figure 2J, FNIP1 and ninein colocalize at the same centrosome.

To evaluate the role of FNIP1 in development we aimed to generate a zebrafish model for Fnip1 loss. Using comparative genomics we identified a zebrafish protein with 67\% identity to human FNIP1 (ENSDARP00000090222, ensembl Zv9, Figure S4A). Using genome-wide analysis of the zebrafish transcriptome from Nepal et al. (15), we determined that zebrafish fnip1 is maternally present in fertilized eggs and during the first cell cycles (Figure 3A and B). At subsequent stages fnip1 is expressed at lower levels throughout the whole embryo and is increasing from $1 \mathrm{k}$ cell stage to 48 hours post-fertilisation (hpf). We confirmed this and determined any spatial restriction by whole-mount in situ hybridization (Figure 3C and D). At $24 \mathrm{hpf}$ expression is most pronounced in the lens, the mesenchyme of the pectoral fin bud and the brain, in particular the proliferative zone of the posterior midbrain and in parts of the hindbrain (Figure 3E). In addition, fnip 1 is expressed in the myotome (Figure 3F). At 50 hpf fnip1 is also expressed in the hatching gland and the fin (Figure 3G-J). fnip1 is transcribed by the embryo at least up to larval stages (Figure 3A and B). 
CHAPTER 4
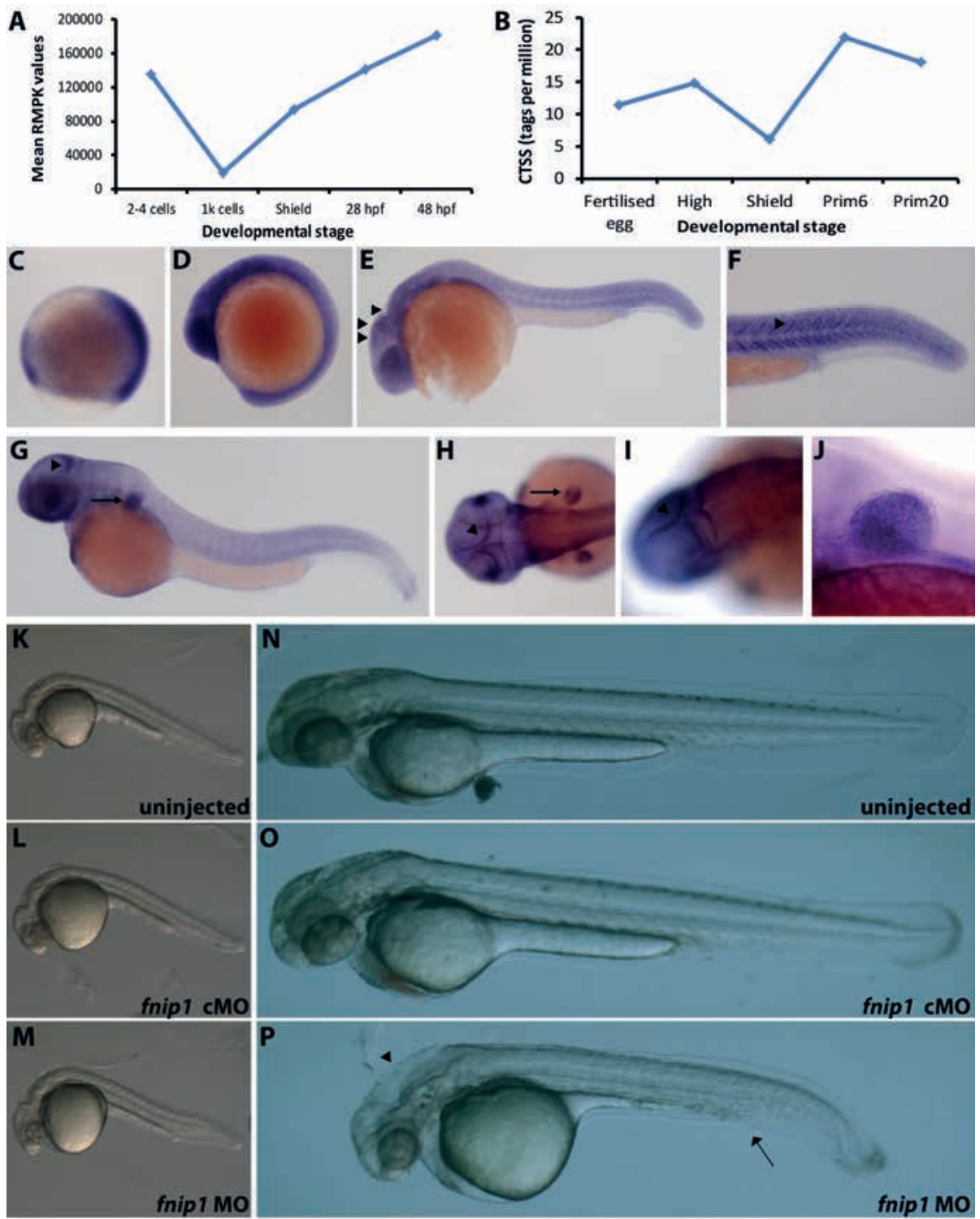

Figure 3 - fnip1 is required for zebrafish embryonic development

(A) RNA sequencing reads per kb per million (RPMK) values for zebrafish fnip1 at indicated stages extracted from our published zebrafish transcriptome database (15). (B) Graphs showing cap analysis of gene expression (CAGE) transcription start site values (CTSS) for zebrafish fnip1 at indicated stages extracted from our published zebrafish transcriptome database (15).

(C-J) Spatial and temporal expression of fnip1 in early zebrafish embryos using whole mount RNA in situ hybridization. (C) Side view of a 75\% epiboly stage ( 8 hours post fertilization (hpf) embryo with low level expression in the whole embryo $(\mathrm{n}=1$, e = 10). (D) Side view of an 11 somite stage (14.3 hpf) embryo with low level expression in the whole embryo $(n=1$, e $=10)$. (E) Side view of a 24 hpf stage embryo showing low level expression in the whole embryo with increased expression in the brain, in particular the tectum, posterior midbrain and hindbrain (indicated by arrowheads). Increased expression is also seen in the myotome $(n=2, e=20)$. (F) Detailed side view of the tail of a $24 \mathrm{hpf}$ 
As the c.1989delT mRNA is degraded by nonsense-mediated decay, we decided on a zebrafish knockdown model to examine the effects of Fnip1 loss. We used morpholino antisense oligonucleotides directed against the acceptor site of fnip1's second coding exon (Figure S4B) to induce splicing defects of the pre-mRNA as verified by RT-PCR (Figure S4C), predicted to lead to a frameshift and premature stop codon in exon 4 . An eight basepair mismatch morpholino served as a control for off-target effects. Injection of the splice-blocking morpholino into zebrafish embryos resulted in a two-hour delay in development and cell death in the brain of 24 hpf morphants (Figure 3K-M). At 48 hpf, the morphant embryos displayed a more severe phenotype, characterized by shortened body axis and curvature, cardiac edema, defects in tail circulation, larger yolk and thinner yolk extension, small eyes and hydrocephalus (Figure 3N-P). Expression of fnip1 was retained in all tissues affected in the morphants as detected by whole mount in situ hybridization. Interference with this expression in particular in the growth zone of the brain may explain the hydrocephalus. Although rescue experiments using coinjection of capped RNA made in vitro from zebrafish fnip1 cDNA were ineffective, introduction of five mismatches into the fnip1 morpholino (control cMO) completely abolished the observed defects, suggesting the observed phenotypes are the result of fnip1 morpholino injection.

BHD patients develop kidney cysts and have an increased risk of renal neoplasia (3). In addition, the hydrocephalus phenotype is frequently seen in embryos with kidney defects $(16,17)$. This prompted us to search for pronephric abnormalities by injecting the morpholino into $\mathrm{Tg}(w t 1 b$ :EGFP) zebrafish embryos, which express EGFP exclusively in the pronephros and exocrine pancreas (18). As shown in Figure S4D-E, there were no obvious pronephric defects in morphants at $48 \mathrm{hpf}$ as compared to mismatch-injected embryos. In addition, we investigated the effect of fnip1 knockdown on cilia in vivo. Whole mount immunofluorescence for acetylated alpha tubulin (a well-known ciliary marker (19)) revealed no obvious difference in cilia number or appearance in morphant embryos compared to mismatch-injected controls (Figure S4F-G). In accordance with our in vitro data this indicates that fnip1 deficiency is unlikely to lead to a ciliopathy.

embryo stage showing expression in the myotome (arrowhead). (G) Side view of a 50 hpf stage embryo showing low level expression in the whole embryo with increased expression in the posterior tectum (arrowhead), hatching gland (black arrow) and fin (white arrow, $n=2, e=20$ ). $(\mathrm{H})$ Dorsal view of a 50 hpf embryo showing expression in the posterior tectum (arrowhead) and fin (arrow). (I) Detailed dorsal view of fnip1 expression in the posterior tectum of a $50 \mathrm{hpf}$ embryo (arrow head). (J) Detailed side view of fnip1 expression in the fin of a 50 hpf embryo.

(K-P) Phenotype analysis of fnip1 morphant embryos $(\mathrm{n}=4 \mathrm{e}=80)$. (K) Uninjected, $24 \mathrm{hpf} \mathrm{AB}^{*} \mathrm{wt}$ embryo. (L) $24 \mathrm{hpf}$ embryo injected with mismatch morpholino (cMO), indistinguishable from the uninjected embryos. (M) 24 hpf embryo injected with fnip1 morpholino (MO), showing cell death in the brain and a two-hour developmental delay. (N) Uninjected, 48 hpf AB* wt embryo. (O) 48 hpf embryo injected with mismatch morpholino, indistinguishable from the uninjected embryos. (P) 48 hpf embryo injected with fnip1 morpholino, showing a delay in development, larger yolk and thinner yolk extension, curved body axis, problems in tail circulation (arrow), cardiac edema, small eyes and hydrocephalus (arrowhead). ( $\mathrm{n}=$ number of separate experiments, $\mathrm{e}=$ number of embryos) 
CHAPTER 4
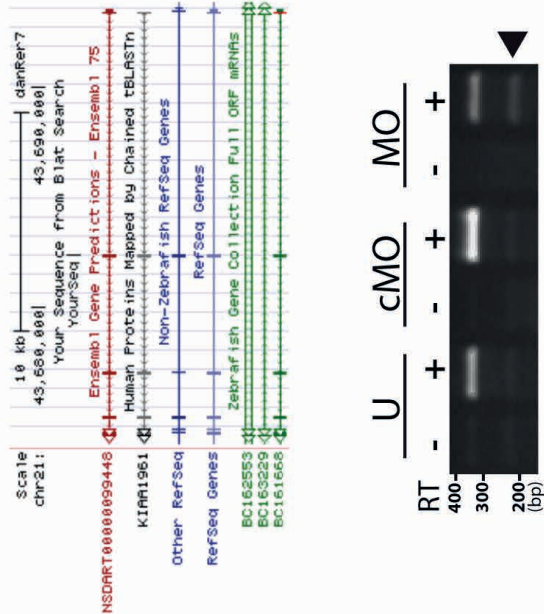

$\infty$

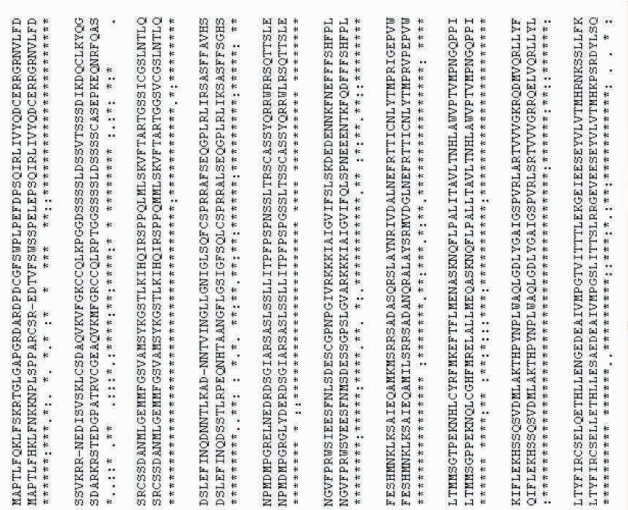

\|n \|\|\|\|\|\|\|\|$\|$
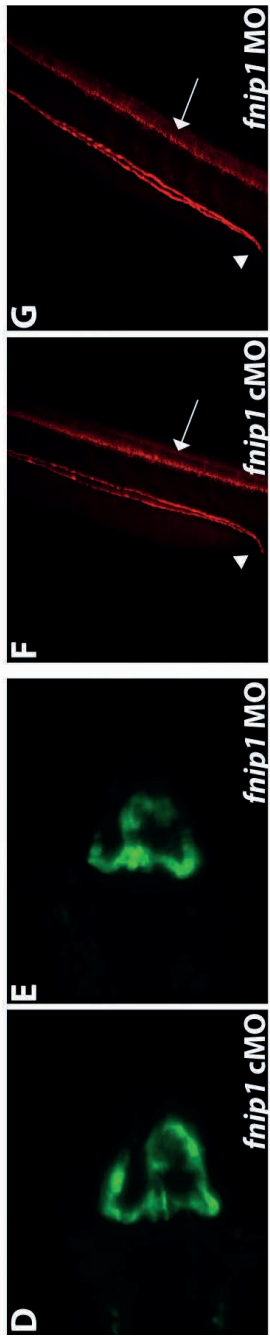

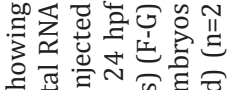

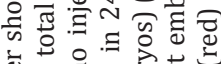

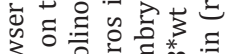

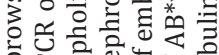

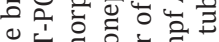

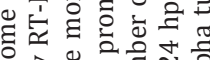

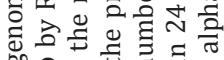

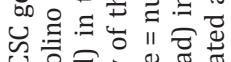

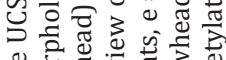
ङ

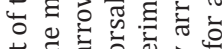

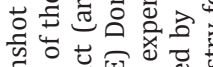

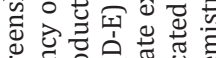

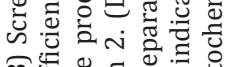

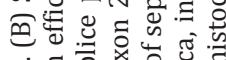
穿令

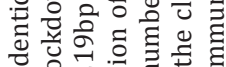
으워

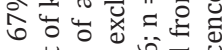
힐.

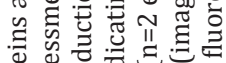

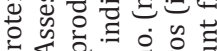

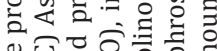

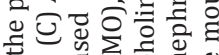

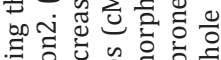

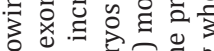

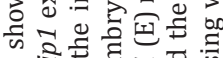

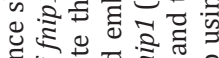

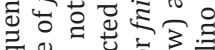
ई

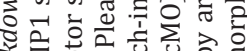
z $\overline{\text { o }}$ 的

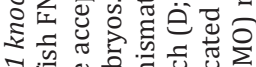

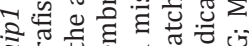

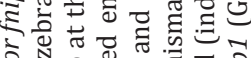

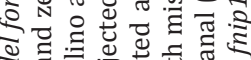
ฐँ

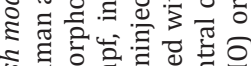

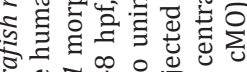

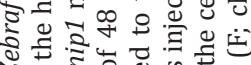
N

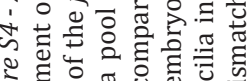
ปै छี

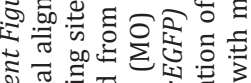

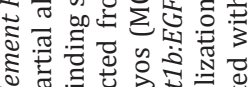

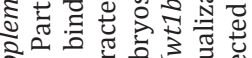

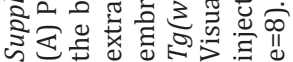


In summary, we show that a c.1989delT germline mutation in FNIP1 causes FMDF. All patients identified so far are heterozygous and the mutant mRNA is degraded by nonsense-mediated decay. This suggests that haploinsufficiency is the causative mechanism of discoid fibroma development. The disorder seems to have been introduced by a single founder and may be unique to a particular population isolate within The Netherlands.

Knockdown of fnip1 in zebrafish embryos suggests that Fnip1 may be important for development. In contrast, mouse models with homozygous knockout of FNIP1 show no defects apart from a reduction in spleen size caused by a block in B cell development $(20,21)$. Although Fnip1 deficiency causes abnormalities in these experimental animal models, they do not explain the human phenotype. The differences in severity between the human, murine and zebrafish phenotypes could be due to differences in timing of FNIP1 deficiency. The zebrafish model studies the developmental effects of reduced Fnip1 levels, while the patients progress normally through embryonic development and the discoid fibromas develop during childhood. In induced pluripotent stem cells the longer, canonical FNIP1 isoform predominates, while after differentiation of these cells into fibroblasts, a shorter isoform is expressed (22), suggesting FNIP1 might have different roles during development compared to after differentiation. Alternatively, there could be differences in FNIP1 function between species, possibly as a result of functional divergence between FNIP1 and its highly similar paralog FNIP2.

The fnip1 morphant phenotype is highly similar to the phenotype of flcn morphants (E.K. F.M., unpublished data), both showing reduced size of the embryo, brain edema and defects in tail circulation and yolk. In addition, zebrafish flcn and fnip1 have similar expression patterns throughout embryonic development (Figure 3D and E.K. F.M., unpublished data), suggesting that flcn and fnip1 share genetic pathways. Recent reports show that FLCN is a key regulator of numerous cellular processes and many of its functions have been suggested to depend on or be facilitated by FNIP1, including FLCN binding to AMPK subunits, FLCN phosphorylation at serine 62, lysosomal recruitment, Rag and GABARAP interactions and regulation of TFE3 nuclear localization (8, 23-27). In addition, both FNIP1 and FLCN were reported to contain a DENN (differentially expressed in normal versus neoplastic cells) module, which is a guanine exchange factor (GEF) for Rab GTPases $(28,29)$.

However, the in vitro data presented here does not support these functional similarities. Although we show that, similar to FLCN (12), FNIP1 is a ciliary protein, no clear defects in ciliogenesis were found after FNIP1 knockdown either in vitro or in vivo. Thus, we propose that, while BHD is a ciliopathy, the disorder caused by FNIP1 mutation is not. Furthermore, FNIP1 has a distinct centrosomal localization. FLCN is a constitutive component of the centrosome (12), while FNIP1 localizes to ninein-associated centrosomal structures only and in a temporal manner. This 
suggests FNIP1 may have a function similar to ninein. Ninein is a part of the pericentriolar material that surrounds the mother centriole, where it anchors microtubules to subdistal appendages (14). Centriole duplication during S and G2 phase of the cell cycle results in asymmetry between the centrosomes and pericentriolar material of mother and daughter (30). Localization of ninein to specific parts of the centrosome is associated with centriole maturation (31). Similarly, FNIP1 might be a novel component of particular centrosomal structures, regulating centrosome asymmetry and maturation.

These in vitro observations suggesting different functions for FLCN and FNIP1 are supported by the variation in phenotype resulting from FLCN and FNIP1 mutations in humans. Although the clinical presentation of the fibrofolliculomas and discoid fibromas is quite similar, histologically they are distinct. In addition, none of the FMDF patients show any extracutaneous manifestations, apart from one case of pneumothorax according to family history, suggesting that FNIP1 is only critical in skin. In other tissues, FNIP1 haploinsufficiency might be tolerated, or the mutant protein might be expressed at higher levels than in the skin. FNIP1 Val663fsX has impaired binding to FLCN, but not to FNIP2 (Figure S2) and might thus retain partial functionality. An alternative explanation for the lack of systemic phenotype might be compensation by FNIP2. FNIP1 has been reported to be expressed at low levels in the lung and kidney $(9,10)$, while FNIP2 is expressed at higher levels in these organs.

Together these findings represent the first human disorder caused by a mutation in one of the folliculin interacting proteins, a pathophysiology distinct from BHD. 
A FNIP1 mutation causes familial multiple discoid fibromas

\section{Materials and methods}

\section{Ascertainment of patients}

The index patient of family 24 had been referred for skin lesions possibly due to BHD, but showing a trichodiscoma aspect only and childhood onset of lesions. Comparable features were found in families 45 and 50, which originated from the same village as family 24 (5). Previously, we had investigated families 11, 17, 20 and 38 for possible BHD, found no FLCN germline mutations and observed a trichodiscoma-only aspect with early onset of skin lesions compatible with familial multiple trichodiscomas (32). We recently re-examined the index patient described by Starink et al. in 1985 (family T, now numbered family 69) and evaluated clinical data of a FMDF family investigated previously (family $\mathrm{H}$, now numbered family 94). In summary, familial multiple trichodiscomas, now renamed to familial multiple discoid fibromas, was clinically diagnosed in nine families.

\section{Segregation and haplotype analysis}

The FLCN locus was previously excluded by segregation analysis in two separate families, families 11 and 45 (5). Segregation analysis for the FNIP1 and FNIP2 loci was performed for families 11, 24, and 45 using polymorphic short tandem repeat markers distal and proximal to both genes. Short tandem repeat markers D4S2394, D4S1644, D4S1625, D4S413, D4S2997, D4S1090, D4S2368, D4S2431, D4S2417, D5S1453, D5S2501, D5S1505, D5S2098, D5S2120, D5S2057, D5S2002, D5S2117, D5S816, D5S1480, D5S820, and D5S1471 were amplified by standard procedures with one of the primers labeled with a fluorescent FAM (carboxyfluorescein) label. Fragment length was analyzed on an ABI 3130 Genetic Analyzer (Applied Biosystems, Foster City, CA). Haplotype analysis was performed by analyzing 27 FMDF cases and 5 unaffected first degree relatives using markers D5S1505, D5S2098, D5S2120, D5S2057, D5S2002, D5S2117, D5S816, and D5S1480 surrounding the FNIP1 locus.

\section{FNIP1 sequence analysis}

Polymerase chain reaction (PCR) primers were designed to cover all 18 exons and exon/intron boundaries of FNIP1 (primer sequences are available upon request). PCR products were sequenced using the BigDyeTerminator Cycle sequencing kit (Applied Biosystems) and an automated sequencer (ABI 3730 DNA analyzer). Sequencing data was analyzed using Mutation Surveyor software (www.softgenetics.com) and by visual inspection.

\section{Cell lines and cell culture}

Murine inner medullary collecting duct cells (IMCD3) were a kind gift from Dr. R. Giles (Department of Department of Nephrology and Hypertension, University Medical Centre Utrecht, The Netherlands). Human kidney cells (HK-2) were provided by prof. dr. M. van Engeland (Department of Pathology, Maastricht 


\section{CHAPTER 4}

University Medical Center). Primary skin fibroblasts with FNIP1 mutation were obtained from a skin biopsy taken from a consenting 27-year-old male subject diagnosed with FMDF. Normal human dermal fibroblasts (NHDF $\alpha$ ) purchased from Cascade Biologics (Life Technologies) were used as a control.

All cells were cultured on plastic dishes in Dulbecco's Modified Eagle Medium containing $4.5 \mathrm{~g} / \mathrm{l}$ D-glucose and L-glutamine (Lonza, Walkersville, MD, USA) supplemented with $10 \%$ FCS (Lonza) and $50 \mu \mathrm{g} / \mathrm{ml}$ gentamycin (Lonza) at $37^{\circ} \mathrm{C}$ in $5 \%$ CO2 (humidified atmosphere). Medium was changed every four days. At confluence, cells were split using trypsin-EDTA.

Human sperm was obtained from volunteer donors (healthy men with no known fertility problems randomly selected from the general public) who were recruited in accordance with the HFEA Code of Practice (version 8) under ethical approval (08/S1402/6) from the EoSRES REC1. Capacitated sperm cells were prepared using a swim-up assay (33) and were then immediately processed for immunofluorescence by spreading $10 \mathrm{ml}$ sperm solution onto Polysine slides (VWR, Leicestershire, UK). Slides were air-dried prior to fixation in Methanol:Acetone (1vol:1vol) for $20 \mathrm{~min}$ at $-20^{\circ} \mathrm{C}$ and staining was performed as described for immunocytochemistry.

\section{DNA constructs, transfections and antibodies}

Human FNIP1 wild-type was amplified by PCR using the primers in Table S1. The PCR product was cloned into pEGFP-c1 (Clontech, Mountain View, CA) using XhoI and BamHI and subsequently cloned into pQCXIB (Clontech) using NotI and PacI. EGFP-tag was exchanged for 3xHA-tag or V5-tag. Tagged versions of FNIP1 mutant Val663fs were generated by site-directed mutagenesis using the Quikchange II Site Directed Mutagenesis kit according to the manufacturer's instructions (Agilent, Santa Clara, CA, USA). For primers see Table S1.

The myc-FNIP2 plasmid in the pCAGGS backbone was a kind gift from Professor O. Hino (Juntendo University School of Medicine, Japan). Human specific FNIP1 shRNA plasmids pLKO.1-puro-shRNA9563 and pLKO.1-puro-shRNA9412 were purchased from Sigma (St. Louis, USA).

Primary antibodies used for immunofluorescence microscopy were custom made rabbit antibodies against the N-terminal peptide (RTGLGAPGRDARDPDC) of human FNIP1 (NP_588613) generated and affinity purified by Eurogentec (Seraing, Belgium [1:50]), anti-FNIP1 (rabbit monoclonal, ab134969 Abcam, Cambridge, UK [1:1000]), anti-FLCN-AP (rabbit polyclonal, a kind gift from Prof. A. Pause, [1:200]), anti-acetylated-alpha-tubulin (mouse, T7451 Sigma, [1:1000]), anti-pericentrin (rabbit polyclonal, ab4448 Abcam [1:1000]) and anti-gammatubulin (mouse monoclonal, T3559 Sigma [1:1000]). Secondary antibodies used were goat-anti-mouse Texas red (1010-07 Southern Biotech, Birmingham, AL, USA, [1:80]) and goat-anti-rabbit FITC (4050-02 Southern Biotech, [1:80]). Primary antibodies used for immunoprecipitation were anti-HA (rat monoclonal 11867423001 Roche, Basel, Switzerland), anti-V5 (mouse monoclonal, 46-0705 
Life Technologies, Paisley, UK) and anti-myc (mouse clone 9E10, M5546 SigmaAldrich). Primary antibodies used for Western blot were raised against FNIP1 (ab134969 Abcam, Cambridge, UK [1:1000]), FLCN (rabbit monoclonal, 3697 Cell Signalling, Danvers, MA, USA [1:5000]), actin (mouse monoclonal, A5441Sigma [1:30,000]). Secondary antibodies used include HRP-conjugated goat-anti-mouse and goat-anti-rabbit (Jackson ImmunoResearch Laboratories, West Grove, PA, USA, [1:10000]).

Supplement Table S1 - Primer and morpholino sequences

\begin{tabular}{ll}
\hline cloning FNIP1 fw & 5'tctcgagctatggccctacgctgttccag3' \\
cloning FNIP1 rv & 5'cagatcttaaaggagtatttgtgcaac3' \\
Mutagenesis fw & 5'tcttaatttatctctgtactgtttacatcaacagcattttcttcttgg3' \\
Mutagenesis rv & 5'caagaagaaaatgctgttgatgtaaacagtacagagataaattaaga3' \\
RT-PCR FNIP1 fw & 5'ttgcagtcatccactccttg3' \\
RT-PCR FNIP1 rv & 5'aaccactcccagctccttga3' \\
Sequencing c.1989delT & 5'ccacaaccattcctgtggat3' \\
fnip1 probe1 fw & 5'actcggatgccagaaagaga3' \\
fnip1 probe1 rv & 5'TAATACGACTCACTATAGGGaggctgtcctgcagagtgtt3' \\
fnip1 probe2 fw & 5'cggacaagagctttccagac3' \\
fnip1 probe2 rv & 5'TAATACGACTCACTATAGGGaacctccaaacaacgacctg3' \\
fnip1 morpholino & 5'agctgaggaaaacacacatgcacaa3' \\
fnip1 mismatch & 5'GgTCAGCaaaacacaGatCcacaa3' \\
RT-PCR fnip1 fw & 5'ggccccacattatttcata3' \\
RT-PCR fnip1 rv & 5'gaagcggttttgttctttgg3' \\
\hline
\end{tabular}

Transfection and generation of stable cell lines

Transient transfections were performed using Lipofectamine 2000 according to the manufacturer's protocol (Life Technologies). HK-2 cells with stable knockdown of FLCN and non-target controls have been described previously (12). To generate FNIP1 knockdown cell lines, HK-2 cells were transfected with appropriate plasmid DNA. 24 hours after transfection selection of $2 \mu \mathrm{g} / \mathrm{ml}$ puromycin (Invivogen) was added. Clonal cell populations were picked using cloning cylinders and subsequently analyzed from FNIP1 expression by Western blot and qPCR (using FNIP1 QuantiTect Primer Assay primer sets, purchased from Qiagen, who reserve the right to withhold primer information).

Transient transfections were performed using Lipofectamine 2000 according to the manufacturer's protocol (Life Technologies). HK-2 cells with stable knockdown of FLCN and non-target controls have been described previously (12). To generate FNIP1 knockdown cell lines, HK-2 cells were transfected with appropriate plasmid DNA. 24 hours after transfection, selection was added $(2 \mu \mathrm{g} / \mathrm{ml}$ puromycin (Invivogen)). Clonal cell populations were picked using cloning cylinders and subsequently analyzed by qPCR and Western blot for FNIP1 expression. 


\section{CHAPTER 4}

\section{Immunocytochemistry}

For fluorescent microscopy cells were cultured on coverslips. Cilium formation was induced by culturing the cells for at least four days after reaching confluence or by serum starving the cells for indicated amount of hours. When ready for staining, cells were fixed using either ice-cold $100 \%$ methanol or $4 \%$ paraformaldehyde at room temperature and permeabilized using $0.2 \%$ Triton X100, depending upon the primary antibody. To block non-specific binding sites, cells were incubated in 3\% BSA in TBS block buffer for 20 minutes. Next, cells were incubated overnight at $4{ }^{\circ} \mathrm{C}$ in primary antibodies, appropriately diluted in block buffer, followed by 1 hour incubation at room temperature in secondary antibody. DNA was stained with DAPI. Fluorescent samples were visualized using a Leica TCS SPE confocal laser scanning fluorescence microscope (Leica DMRBE, Mannheim, Germany). Images were further processed using ImageJ software (NIH).

The percentage of ciliated cells $(n=2$, average 100 cells per group per experiment for the fibroblasts, $n=4$ average 150 cells per group per experiment for the HK-2) was analyzed using a two-tailed Pearson's chi-square test for categorical data (significant $\mathrm{p}<0.05$ ).

\section{Co-immunoprecipitation}

HEK293 cells transiently transfected with appropriate plasmids were lysed in BHD lysis buffer (20 mM Tris, $135 \mathrm{mM} \mathrm{NaCl}, 5 \%$ (v/v) glycerol, $50 \mathrm{mM} \mathrm{NaF}$ and $0.1 \%$ (v/v) Triton X-100, pH 7.5 supplemented with protease inhibitors), centrifuged to remove particulate matter and protein quantified using Bradford reagent (SigmaAldrich). Anti-HA and anti-V5 coupled to protein G-Sepharose beads (GE Healthcare Life Sciences, Little Chalfont, UK) were used to immunoprecipitate HAand V5-tagged proteins as appropriate. Immunoprecipitates were washed three times in lysis buffer and resuspended in NuPAGE LDS sample buffer (Life Technologies, Paisley, UK).

\section{Western blotting}

Cells were harvested using typsin-EDTA and lysed in NP40 lysis buffer (150 mM $\mathrm{NaCl}, 1 \% \mathrm{NP}-40,250 \mathrm{mM}$ Tris $\mathrm{pH}$ 7.3) supplemented with protease and phosphatase inhibitors (Roche). Protein concentration was determined using the Bradford protein assay (Sigma). Equal amounts of total protein were subjected to SDS-PAGE electrophoresis before transfer to an Amersham HybondTM-PVDF membrane (GE Healthcare Life Sciences, Buckinghamshire, UK). Membranes were incubated overnight at $4{ }^{\circ} \mathrm{C}$ in primary antibodies diluted in $0.5 \%$ BSA in TBST block buffer, followed by an 1 hour incubation in HRP-conjugated secondary antibodies diluted in 5\% milk in TBST. Signal was detected using enhanced chemiluminescence (ECL) system (Thermo Scientific, Waltham, Massachusetts, USA). 
Nonsense-mediated decay assay

NHDF $\alpha$ and FMDF were cultured with or without $200 \mathrm{ug} / \mathrm{ml}$ puromycin for six hours and total RNA was isolated using High Pure RNA Isolation Kit (11828665001 Roche) according to the manufacturer's instructions. The RNA quality was verified by gel electrophoresis, followed by cDNA synthesis from $1 \mu \mathrm{g}$ total RNA using reverse transcriptase M-MLV (M368, Promega) with oligodT primers. fnip1 cDNA was amplified using primers spanning five exons to avoid genomic DNA contamination (Table S1) and the area around the mutation was sequenced.

fnip1 expression analysis in zebrafish embryos

Whole-mount in situ hybridization was carried out as described by Thisse and Thisse (34). For fnip1 gene probes PCR products were amplified from fnip1 template cDNA purchased from Source BioScience (Nottingham, UK) in pCMVSPORT6.1 using the primers in Table S1. The antisense RNA probes were transcribed using T7 mMESSAGE mMACHINE kit (Ambion, Austin, USA) with Dig labelling mix (Roche). Embryos at pre-tail bud stage were fixed in 4\% paraformaldehyde overnight and then manually dechorionated. Embryos post 24 hours post fertilisation (hpf) were manually dechorionated and then fixed in $4 \%$ paraformaldehyde overnight at $4^{\circ} \mathrm{C}$. Embryos were cleared in glycerol and imaged with a Nikon SMZ800 stereo microscope and Canon Eos 1100D camera and software.

\section{Zebrafish embryo fnip1 morphant phenotyping}

To knockdown fnip1 in zebrafish embryos a morpholino antisense oligonucleotide (Genetools, Philomath, OR, USA) was designed to target the acceptor site of the second coding exon (morpholino MO), predicted to cause splicing defects of the mRNA. As a control an eight basepair mismatch morpholino was used (control cM0). For sequences see Table S1. Wild-type $\mathrm{AB}^{*}$ and $\mathrm{Tg}(w t 1 b: E G F P)(18)$ zebrafish were maintained using standard conditions according to the UK Animals (Scientific Procedures) Act of 1986 in a flow-through system of aerated, charcoal filtered tap water with a 14 hour light/10 hour dark cycle (Tecniplast; UK). Breeding pairs were set up in breeding cages the day before collection of embryos. Embryos were obtained from crosses the following morning and injected with a solution containing $50 \mu \mathrm{M}$ morpholino or mismatch, $0.1 \%$ phenol red (Sigma) and $15 \mathrm{ng} / \mu \mathrm{l}$ CFP RNA at single-cell stage using a microinjector (Tritech research, Los Angeles, CA, USA). Embryos were maintained at $28^{\circ} \mathrm{C}$ in E3 embryo medium containing $0.003 \%$ phenylthiourea (Sigma) to prevent pigmentation. Embryos were staged according to Kimmel et. al. (35) and at $18 \mathrm{hpf}$ embryos expressing CFP were selected. For assessment of morphological phenotype the $24 \mathrm{hpf}$ and $48 \mathrm{hpf}$ fish larvae were anaesthetized in $0.3 \mathrm{mg} / \mathrm{ml}$ ethyl-3 aminobenzoate methanesulfonate (Sigma) and imaged with a Nikon SMZ 1500 stereo microscope or a Leica TCS LSI confocal. 


\section{CHAPTER 4}

Efficiency of the morpholinos was verified by RT-PCR on total RNA isolated from a pool of $48 \mathrm{hpf}$, injected embryos using Trizol reagent (Ambion) according to the manufacturer's instructions. RNA was treated with TURBO DNAse (Ambion) and cDNA was produced using reverse transcriptase M-MLV (Promega, Fitchburg, USA) with random hexamer primers (Fermentas, Vilnius, Lithuania). For detection of splice defects of exon2 specific PCR for fnip1 was performed on $5 \mu$ l cDNA using GoTaq polymerase (Promega) using a forward primer in exon 1 and reverse primer in exon 3 (Table S1) using the following parameters: $94^{\circ} \mathrm{C}$ for $5 \mathrm{~min},\left(94^{\circ} \mathrm{C}\right.$ for $30 \mathrm{sec} \rightarrow 59^{\circ} \mathrm{C}$ for $40 \mathrm{sec} \rightarrow 72^{\circ} \mathrm{C}$ for $30 \mathrm{sec}$ ) $\mathrm{x} 30$ and $72^{\circ} \mathrm{C}$ for $5 \mathrm{~min}$.

\section{Zebrafish embryo fnip1 morphant cilia analysis}

For whole mount immunohistochemistry 24 hpf embryos injected with either mismatch or morpholino were fixed in 4\% paraformaldehyde in PBS overnight at $4^{\circ} \mathrm{C}$. Embryos were then washed in PBST, permeabilized with $10 \mu \mathrm{g} / \mathrm{ml}$ proteinase $\mathrm{K}$ for 20 minutes and refixed for 20 minutes at room temperature. Following block in $10 \%$ Newborn Calf Serum (NCS) for at least 1 hour, embryos were incubated in primary anti-acetylated- $\alpha$-tubulin (mouse, ab24610 Abcam [1:200]) in block buffer overnight at $4^{\circ} \mathrm{C}$. Next, embryos were washed in PBST four times 30 minutes and incubated in sheep-anti-mouse cy3-conjugated antibodies (Sigma C2181 [1:200]) in block buffer overnight at $4^{\circ} \mathrm{C}$. After removal of secondary antibodies, embryos were washed in PBST four times 30 minutes each and transferred into Vectorshield (Vector Laboratories) at $4^{\circ} \mathrm{C}$.

\section{Supplemental data}

Supplemental Data includes four figures and one table.

\section{Acknowledgements}

ML is supported by the Dutch Cancer Society KWF, the Association for International Cancer Research AICR (11-0687) and the Annadal Foundation. MvS is supported by a grant from the Dutch Cancer Society KWF (UM2009-4352). F.M., A.R.T. and S.S are supported by the Myrovlytis Trust. 


\section{References}

1. Birt AR, Hogg GR, Dube WJ. Hereditary multiple fibrofolliculomas with trichodiscomas and acrochordons. Archives of dermatology. 1977 Dec;113(12):1674-7. PubMed PMID: 596896.

2. Menko FH, van Steensel MA, Giraud S, Friis-Hansen L, Richard S, Ungari S, et al. BirtHogg-Dube syndrome: diagnosis and management. The lancet oncology. 2009 Dec;10(12):1199-206. PubMed PMID: 19959076.

3. Nickerson ML, Warren MB, Toro JR, Matrosova V, Glenn G, Turner ML, et al. Mutations in a novel gene lead to kidney tumors, lung wall defects, and benign tumors of the hair follicle in patients with the Birt-Hogg-Dube syndrome. Cancer cell. 2002 Aug;2(2):15764. PubMed PMID: 12204536.

4. Starink TM, Kisch LS, Meijer CJ. Familial multiple trichodiscomas. A clinicopathologic study. Archives of dermatology. 1985 Jul;121(7):888-91. PubMed PMID: 4015134.

5. Starink TM, Houweling AC, van Doorn MB, Leter EM, Jaspars EH, van Moorselaar RJ, et al. Familial multiple discoid fibromas: a look-alike of Birt-Hogg-Dube syndrome not linked to the FLCN locus. Journal of the American Academy of Dermatology. 2012 Feb;66(2):259 e1-9. PubMed PMID: 21794948.

6. Gijezen LM, Vernooij M, Martens H, Oduber CE, Henquet CJ, Starink TM, et al. Topical rapamycin as a treatment for fibrofolliculomas in Birt-Hogg-Dube syndrome: a doubleblind placebo-controlled randomized split-face trial. PloS one. 2014;9(6):e99071. PubMed PMID: 24910976. Pubmed Central PMCID: 4049818.

7. van Steensel MA, Verstraeten VL, Frank J, Kelleners-Smeets NW, Poblete-Gutierrez P, Marcus-Soekarman D, et al. Novel mutations in the BHD gene and absence of loss of heterozygosity in fibrofolliculomas of Birt-Hogg-Dube patients. The Journal of investigative dermatology. 2007 Mar;127(3):588-93. PubMed PMID: 17124507.

8. Baba M, Hong SB, Sharma N, Warren MB, Nickerson ML, Iwamatsu A, et al. Folliculin encoded by the BHD gene interacts with a binding protein, FNIP1, and AMPK, and is involved in AMPK and mTOR signaling. Proceedings of the National Academy of Sciences of the United States of America. 2006 Oct 17;103(42):15552-7. PubMed PMID: 17028174. Pubmed Central PMCID: 1592464.

9. Takagi Y, Kobayashi T, Shiono M, Wang L, Piao X, Sun G, et al. Interaction of folliculin (Birt-Hogg-Dube gene product) with a novel Fnip1-like (FnipL/Fnip2) protein. Oncogene. 2008 Sep 11;27(40):5339-47. PubMed PMID: 18663353.

10. Hasumi H, Baba M, Hong SB, Hasumi Y, Huang Y, Yao M, et al. Identification and characterization of a novel folliculin-interacting protein FNIP2. Gene. 2008 May 31;415(1-2):60-7. PubMed PMID: 18403135. Pubmed Central PMCID: 2727720.

11. Lim TH, Fujikane R, Sano S, Sakagami R, Nakatsu Y, Tsuzuki T, et al. Activation of AMPactivated protein kinase by MAPO1 and FLCN induces apoptosis triggered by alkylated base mismatch in DNA. DNA repair. 2012 Mar 1;11(3):259-66. PubMed PMID: 22209521.

12. Luijten MN, Basten SG, Claessens T, Vernooij M, Scott CL, Janssen R, et al. Birt-HoggDube syndrome is a novel ciliopathy. Human molecular genetics. 2013 Nov 1;22(21):4383-97. PubMed PMID: 23784378. Pubmed Central PMCID: 3792695.

13. Hildebrandt F, Benzing T, Katsanis N. Ciliopathies. The New England journal of medicine. 2011 Apr 21;364(16):1533-43. PubMed PMID: 21506742. Pubmed Central PMCID: 3640822.

14. Mogensen MM, Malik A, Piel M, Bouckson-Castaing V, Bornens M. Microtubule minusend anchorage at centrosomal and non-centrosomal sites: the role of ninein. Journal of cell science. 2000 Sep;113 ( Pt 17):3013-23. PubMed PMID: 10934040. 


\section{CHAPTER 4}

15. Nepal C, Hadzhiev Y, Previti C, Haberle V, Li N, Takahashi H, et al. Dynamic regulation of the transcription initiation landscape at single nucleotide resolution during vertebrate embryogenesis. Genome research. 2013 Nov;23(11):1938-50. PubMed PMID: 24002785. Pubmed Central PMCID: 3814893.

16. Kramer-Zucker AG, Olale F, Haycraft CJ, Yoder BK, Schier AF, Drummond IA. Ciliadriven fluid flow in the zebrafish pronephros, brain and Kupffer's vesicle is required for normal organogenesis. Development. 2005 Apr;132(8):1907-21. PubMed PMID: 15790966.

17. Zhao C, Malicki J. Genetic defects of pronephric cilia in zebrafish. Mechanisms of development. 2007 Aug;124(7-8):605-16. PubMed PMID: 17576052.

18. Perner B, Englert C, Bollig F. The Wilms tumor genes wt1a and wt1b control different steps during formation of the zebrafish pronephros. Developmental biology. 2007 Sep 1;309(1):87-96. PubMed PMID: 17651719.

19. Piperno G, Fuller MT. Monoclonal antibodies specific for an acetylated form of alphatubulin recognize the antigen in cilia and flagella from a variety of organisms. The Journal of cell biology. 1985 Dec;101(6):2085-94. PubMed PMID: 2415535. Pubmed Central PMCID: 2114011.

20. Baba M, Keller JR, Sun HW, Resch W, Kuchen S, Suh HC, et al. The folliculin-FNIP1 pathway deleted in human Birt-Hogg-Dube syndrome is required for murine B-cell development. Blood. 2012 Aug 9;120(6):1254-61. PubMed PMID: 22709692. Pubmed Central PMCID: 3418720.

21. Park H, Staehling K, Tsang M, Appleby MW, Brunkow ME, Margineantu D, et al. Disruption of Fnip1 reveals a metabolic checkpoint controlling B lymphocyte development. Immunity. 2012 May 25;36(5):769-81. PubMed PMID: 22608497. Pubmed Central PMCID: 3361584.

22. Venables JP, Lapasset L, Gadea G, Fort P, Klinck R, Irimia M, et al. MBNL1 and RBFOX2 cooperate to establish a splicing programme involved in pluripotent stem cell differentiation. Nature communications. 2013;4:2480. PubMed PMID: 24048253.

23. Tsun ZY, Bar-Peled L, Chantranupong L, Zoncu R, Wang T, Kim C, et al. The folliculin tumor suppressor is a GAP for the RagC/D GTPases that signal amino acid levels to mTORC1. Molecular cell. 2013 Nov 21;52(4):495-505. PubMed PMID: 24095279. Pubmed Central PMCID: 3867817.

24. Petit CS, Roczniak-Ferguson A, Ferguson SM. Recruitment of folliculin to lysosomes supports the amino acid-dependent activation of Rag GTPases. The Journal of cell biology. 2013 Sep 30;202(7):1107-22. PubMed PMID: 24081491. Pubmed Central PMCID: 3787382.

25. Wang L, Kobayashi T, Piao X, Shiono M, Takagi Y, Mineki R, et al. Serine 62 is a phosphorylation site in folliculin, the Birt-Hogg-Dube gene product. FEBS letters. 2010 Jan 4;584(1):39-43. PubMed PMID: 19914239.

26. Betschinger J, Nichols J, Dietmann S, Corrin PD, Paddison PJ, Smith A. Exit from pluripotency is gated by intracellular redistribution of the bHLH transcription factor Tfe3. Cell. 2013 Apr 11;153(2):335-47. PubMed PMID: 23582324. Pubmed Central PMCID: 3661979.

27. Dunlop EA, Seifan S, Claessens T, Behrends C, Kamps M, Rozycka E, et al. FLCN, a novel autophagy component, interacts with GABARAP and is regulated by ULK1 phosphorylation. Autophagy. 2014;0:0 - -1.

28. Zhang D, Iyer LM, He F, Aravind L. Discovery of Novel DENN Proteins: Implications for the Evolution of Eukaryotic Intracellular Membrane Structures and Human Disease. Frontiers in genetics. 2012;3:283. PubMed PMID: 23248642. Pubmed Central PMCID: 3521125. 
29. Nookala RK, Langemeyer L, Pacitto A, Ochoa-Montano B, Donaldson JC, Blaszczyk BK, et al. Crystal structure of folliculin reveals a hidDENN function in genetically inherited renal cancer. Open biology. 2012 Aug;2(8):120071. PubMed PMID: 22977732. Pubmed Central PMCID: 3438538.

30. Nigg EA, Stearns T. The centrosome cycle: Centriole biogenesis, duplication and inherent asymmetries. Nature cell biology. 2011 Oct;13(10):1154-60. PubMed PMID: 21968988.

31. Ou YY, Mack GJ, Zhang M, Rattner JB. CEP110 and ninein are located in a specific domain of the centrosome associated with centrosome maturation. Journal of cell science. 2002 May 1;115(Pt 9):1825-35. PubMed PMID: 11956314.

32. Leter EM, Koopmans AK, Gille JJ, van Os TA, Vittoz GG, David EF, et al. Birt-Hogg-Dube syndrome: clinical and genetic studies of 20 families. The Journal of investigative dermatology. 2008 Jan;128(1):45-9. PubMed PMID: 17611575.

33. Lefievre L, Nash K, Mansell S, Costello S, Punt E, Correia J, et al. 2-APB-potentiated channels amplify CatSper-induced $\mathrm{Ca}(2+)$ signals in human sperm. The Biochemical journal. 2012 Dec 1;448(2):189-200. PubMed PMID: 22943284. Pubmed Central PMCID: 3492921.

34. Thisse C, Thisse B. High-resolution in situ hybridization to whole-mount zebrafish embryos. Nature protocols. 2008;3(1):59-69. PubMed PMID: 18193022.

35. Kimmel CB, Ballard WW, Kimmel SR, Ullmann B, Schilling TF. Stages of embryonic development of the zebrafish. Developmental dynamics : an official publication of the American Association of Anatomists. 1995 Jul;203(3):253-310. PubMed PMID: 8589427. 



\section{CHAPTER 5}

General discussion 
CHAPTER 5 


\section{General discussion}

The aim of this thesis is to gain more insight into the molecular functioning of FLCN, the protein mutated in Birt-Hogg-Dubé syndrome (BHD), and FLCN's binding partner FNIP1 in order to better understand the pathophysiology of BHD and related disorders.

\section{BHD genetics}

Exactly how mutation of FLCN leads to the BHD phenotype is incompletely understood, but in relation to the development of cancer it has been suggested that FLCN acts as a tumour suppressor. BHD-related tumours in heterozygous animals show a high frequency of second hit mutations or loss of heterozygosity (1-4), supporting the two hit model of tumorigenesis by Knudson (5). Furthermore, reexpression of wild-type FLCN in the UOK257 cell line, derived from a renal tumour from a BHD patient, inhibited tumour formation after subcutaneous injection of the cells into nude mice (6). However, mutations in tumour suppressor genes can also have haploinsufficient or dominant negative effects (7). Thus, FLCN may not act as a classical tumour suppressor in all tissues.

\section{Haploinsufficiency as a causative mechanism for BHD}

Neither second hits nor loss of heterozygosity were detected in either the BHD fibrofolliculomas or the skin nodules of the German Shepherd dog model for BHD $(3,8)$, suggesting that FLCN haploinsufficiency could be the causative mechanism for the uncontrolled growth in the skin. The data presented in Chapter 2 indicate that this also holds true for the initiation of BHD renal lesions. In our in vitro models, which show approximately 50\% knockdown and can therefore be considered haploinsufficiency models, we identified ciliary defects. Ciliary defects can lead to cyst formation (9) and renal cysts are also seen in transgenic mice with heterozygous Flcn knockout $(4,10)$. Similarly, lung cyst formation in BHD could also be the result of FLCN haploinsufficiency. As BHD patients develop multiple cysts at a relatively young age, a second hit may not be necessary.

However, several lines of evidence suggest that haploinsufficiency might not be causative of BHD. Mice with a kidney-specific homozygous knockout of Flcn develop polycystic kidneys and cystic renal cell carcinoma (11, 12). Animals heterozygous for targeted renal Flcn knockout had no phenotype, indicating that development of cystic kidneys requires that both alleles be affected. Additionally, in humans, Smith-Magenis syndrome (SMS; OMIM \#182290) can be caused either by a mutation in RAI1 or by a heterozygous deletion of chromosome $17 \mathrm{p} 11.2$. This region includes the FLCN locus, thus causing FLCN haploinsufficiency $(13,14)$. No increased risk of pneumothorax or renal cancer has been reported in SMS patients $(15,16)$, inconsistent with the idea that BHD is caused by haploinsufficiency. However, the majority of SMS patients described are younger than 25 years, which is before BHD skin and renal symptoms first arise (16). Even if typical skin lesions 
are present, they may not be recognised as BHD marker lesions (17). We therefore suggest that these patients should be followed carefully, with particular interest in BHD-related symptoms.

Could mutant FLCN have a dominant negative effect?

An alternative explanation for development of the cystic BHD phenotype could be a dominant negative effect of mutant FLCN. The mutant version of FLCN may interfere with functioning of the protein produced by the wild-type allele. One of the hallmarks of a dominant negative effect is that partial deletion or mutation of the gene of interest causes more severe defects compared to complete deletion (18). Several BHD patients have been described with a mutation in the translational start codon in exon 4 (c.3delG), predicted to completely inhibit translation $(19,20)$. Yet, these patients develop BHD with a frequency of symptoms similar to BHD patients with FLCN point mutations, suggesting that BHD might not be the result of a dominant negative effect. Conversely, in these patients translation may be initiated from an internal start codon downstream of the original ATG (21). This would lead to production of a truncated protein lacking Nterminal amino acids, possibly causing a dominant negative effect. However, a deletion of the non-coding exon 1 containing the putative FLCN promoter region has been reported in BHD patients (22). Deletion of this exon theoretically completely inhibits protein production (22) and cannot be (partially) rescued by translation from an internal start codon. These patients also develop the full spectrum of BHD manifestations, arguing against a dominant negative effect.

Further evidence against a dominant negative effect comes from observations that mutant FLCN proteins are less stable compared to wild-type $\operatorname{FLCN}(23,24)$. We have not been able to detect FLCN-H429 in UOK257 cells by Western blot (unpublished) and BHD tumours have been reported to have lost expression of FLCN mRNA (25). Nevertheless, using a custom-made antibody directed against the carboxyl-end of FLCN we detected mutant protein in a tumour from a patient with the germline mutation c.499C>T, encoding the truncated protein Q167X which lacks the antibody epitope (Chapter 2 Figure 1). The antibody detects the protein product of the second allele, which has a 15 base pair deletion predicted to result in the deletion of exon 6 (17), leaving the antibody epitope intact.

The intracellular localisation of FLCN mutant proteins in vitro is similar to localisation of wild-type FLCN to the centrosome, cilia and mitotic spindle (Chapter 2 Figure S3 and S4). Possibly the mutant proteins can still interact with (unknown) endogenous proteins responsible for their correct localisation. Alternatively, we could speculate that FLCN homodimerises, arguing in favour of a dominant negative effect, as the mutant protein could then interfere with the functioning of the wild-type via this interaction. These experiments were conducted in HK-2 cells which express endogenous FLCN (Chapter 2 Figure 4), possibly binding the EGFPtagged FLCN mutants, thereby leading to proper localisation. As there is currently no evidence for this theory it would be of interest to perform an in vitro binding 
assay of purified FLCN with various tags. Co-immunoprecipitation experiments can also be used to search for FLCN dimers. In this case, one would preferably use a cell line with complete loss of endogenous wild-type and/or mutant FLCN to cotransfect with multiple constructs encoding FLCN with various tags. UOK257 cells have been suggested to be FLCN-null (26), but we have shown that they still express low levels of wild-type FLCN (Chapter 2 Figure 2). Considering the embryonic lethality of complete FLCN knockout $(2,4,27)$, we would not expect a FLCN-null cell line to be viable. Alternatively, using novel techniques that combine DNA recognition proteins with DNA cleavage domains (e.g. the CRISPR/Cas9 system (28)), it is now possible to insert a monoallelic tag-encoding sequence into any desired location in the genome. After production of tagged, endogenous FLCN from one allele, co-immunoprecipitation can be performed to search for the untagged protein encoded by the remaining allele.

Even though mutant FLCN appears to be expressed in a BHD renal tumour and seems to localise normally in vitro, the mutant protein may not be functional. Our own data show that exogenous expression of wild-type FLCN in immortalised renal cells disrupts ciliogenesis in monolayer and spheroid formation in threedimensional culture, affecting both spheroid and lumen size (Chapter 2 Figure 5). Conversely, exogenous expression of FLCN-K508R does not affect ciliogenesis in monolayer and only mildly affects lumen size formation, suggesting that the mutant protein has lost a large part of its functionality.

However, it is not certain whether the c.1523A>G mutation encoding K508R is pathogenic. It has been detected in a single patient without a BHD family history. This patient has not developed fibrofolliculomas, lung cysts or pneumothorax and presented with bilateral multifocal renal oncocytomas only, which, in retrospect, is not a clinical manifestation typically associated with BHD (Chapter 2). It could therefore be argued that c.1523A $>\mathrm{G}$ is not a pathogenic BHD mutation, or could represent a low penetrance, variable phenotype. It would be of interest then to systematically examine functionality of other FLCN mutants using the spheroid assay. FLCN-K508R might not be representative for the other mutant proteins, which may affect spheroid formation.

Although K508R is of uncertain significance, various studies have shown different disease-causing FLCN mutants to be non-functional in other assays. Multiple defects resulting from FLCN deficiency, such as more rapid cell cycle progression (24) and xenograft tumour growth of UOK257 cells $(6,29)$ were corrected upon reconstitution of wild-type FLCN, but not FLCN mutants. Finally, functioning of FLCN in various pathways was shown to be supported by FNIP1 (30-34). Binding of FLCN to its interactors FNIP1 and FNIP2 requires the carboxylterminus of FLCN $(30,35,36)$. The majority of pathogenic FLCN mutations lead to a premature stop codon, deleting the protein's C-terminus. This impairs FNIP interaction, possibly disrupting FLCN function and arguing against a dominant negative effect. 


\section{CHAPTER 5}

The BHD cystic phenotype might be caused by loss of both alleles

As stated previously, heterozygous kidney-specific knockout of Flcn in mice did not result in any phenotype. Only mice homozygous for targeted renal knockout developed cystic kidneys $(11,12)$, indicating that both alleles need to be lost for the phenotype to develop. Additionally, in homozygous animals FLCN expression was still detected in some cells, possibly due to no or low Cre recombinase expression, but only in cells lining normal looking tubules and not in cysts (11). In a large percentage of the renal cystic lesions of the BHD German Shepherd dog model inactivation of the wild-type allele was detected (3). Furthermore, it was recently stated that fibrofolliculomas do show loss of heterozygosity, but only in the surrounding stromal fibroblasts and not in the epithelial cells (J. Toro, personal communication 2012), which might explain why previous studies were unable to detect it. Investigations of angiofibroma skin tumours of patients with tuberous sclerosis complex similarly report a high frequency of somatic second hit mutations (37). Due to ethical considerations it is difficult to obtain the material to investigate this in other affected tissues from BHD patients. Renal and pulmonary cystic lesions are often asymptomatic and therefore are not surgically removed. It would be interesting to sequence the Flcn locus in the preneoplastic lesions of Nihon rats heterozygous for mutant Flcn (2) or the cystic kidneys of the transgenic mice heterozygous for inactivation of Flcn $(4,10)$. In this way it could be determined if haploinsufficiency is sufficient to initiate cyst formation or whether loss of heterozygosity and/or somatic second hits are present, similar to the renal and pancreatic cysts in von Hippel Lindau disease $(38,39)$.

Although a dominant negative effect of mutant FLCN can presently not be excluded, these data suggest that the BHD cystic phenotype is most likely the result of loss of both FLCN alleles. Cysts are considered as tumour precursor lesions (40). However, cyst formation alone is not sufficient for cancer development, as polycystic kidney disease does not increase the risk of renal cancer (41). While the vast majority of BHD patients have cysts (20), only 20\%-30\% develop cancer (4244). As cancer development is a multi-step process, tumour formation most likely requires additional genetic or epigenetic events currently unknown for BHD. These additional events are possibly induced by environmental factors which could be tissue-specific, explaining why the renal cysts progress into tumours in contrast to the cutaneous and pulmonary lesions. A recent publication by Possik et al. shows how cells with FLCN deficiency are more resistant to energy-depleting stress, such as heat, anoxia, serum starvation and oxidative stress through activation of AMPK (45). Lack of FLCN was shown to influence autophagy and inhibit apoptosis (34, 45), providing a proliferative advantage for FLCN-deficient cells under metabolic stress (46). Considering the unique cellular environment in renal tissue (e.g. hyperosmolarity of the medulla), this advantage could be most pronounced there, enabling tumour formation. 
In conclusion, studies into the genetics of BHD indicate that FLCN is most likely a tumour suppressor gene and that loss of its protein product FLCN causes BHD cysts, which can progress into renal tumours, possibly through additional genetic events. Furthermore, our data show that levels of endogenous FLCN need to be tightly regulated in order for the protein to function properly. Both in vitro (Chapter 2) and in zebrafish embryos (Chapter 3) knockdown and overexpression of FLCN cause substantial phenotypes. In Chapter 2 we provide an explanation for how loss of FLCN might result in cyst formation at the cellular level.

\section{FLCN's function in ciliogenesis}

In Chapter 2 we report BHD as a novel ciliopathy, a disease caused by malfunctioning of the primary cilium. We show that FLCN localises to tubulinbased structures and that FLCN knockdown results in delayed ciliogenesis, with decreased numbers of primary cilia and reduced ciliary length after brief periods of serum starvation.

FLCN might regulate ciliogenesis through monitoring energy status or the cell cycle Ciliogenesis (Figure 1) is a complex and multistep process that is incompletely understood. One possibility is that the decreased percentage of ciliated cells observed in our experiments is an indirect effect of FLCN deficiency. One of the earliest triggers for ciliogenesis is the absence of proliferative stimuli achieved in vitro by serum deprivation (47). FLCN has been reported as a regulator of energy homeostasis and nutrient signalling through mTOR and AMPK signalling (reviewed in $(48,49))$. These pathways promote protein synthesis and cell growth after activation by growth factors and/or amino acids (50). After serum deprivation, UOK257 cells with restoration of a functional FLCN copy using Scaffold/Matrix Attachment Region (S/MAR) DNA vectors showed inhibition of mTOR via absence of phosphorylated 4E-binding protein-1 (4E-BP1) (29). However, this suppression of mTOR signalling induced by serum deprivation was absent in UOK257 cells without FLCN restoration (29). In addition, UOK257 cells have been shown to favour glycolytic rather than lipid metabolism $(46,51)$. Recently, using both Caenorhabditis elegans and mammalian cells, Possik et al. showed that loss of FLCN constitutively activates AMPK, thereby inducing autophagy and providing resistance to metabolic stress (45). Together these data indicate that FLCNdeficient cells could have a proliferative advantage over control cells under the serum starved conditions used to induce cilium formation. As a result, in the absence of serum they would be less prone to exit the cell cycle and enter G0 or G1, a prerequisite for ciliogenesis. As an alternative explanation, ciliogenesis was recently reported to be regulated by autophagy (52). In contrast to Possik et al, Dunlop et al. reported decreased rather than increased autophagic flux as a result of FLCN deficiency (34). This could lead to decreased ciliogenesis as autophagic removal of the ciliopathy protein OFD1 from the centriolar satellites promotes 
primary cilium biogenesis (52). Conversely, the decrease in autophagic flux in FLCN deficient cells might be the result rather than cause of defective ciliogenesis, as abrogation of ciliogenesis has been shown to inhibit autophagy (53).

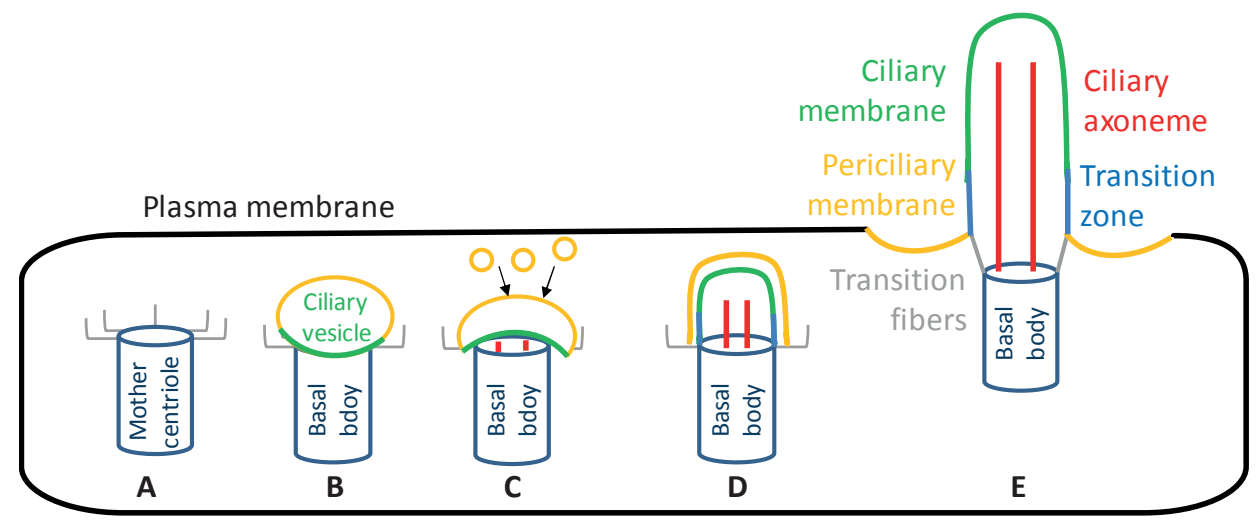

Figure 1 - Schematic representation of ciliogenesis

After the cell enters G1 phase ciliogenesis starts by (A) maturation of the mother centriole into the basal body (dark blue) by acquisition of (sub)distal appendages (grey). (B) The basal body then docks a ciliary vesicle, which (C) flattens into two layers of membrane. The membrane surface grows with the axoneme (red) through fusion with secondary vesicles (yellow). (D) After migration of the basal body/vesicle complex it fuses with the plasma membrane and the outer layer (yellow) will attach to the plasma membrane and (E) the inner layer will form the transition zone (blue) and ciliary membrane (green).

After establishment of cell polarity, ciliogenesis is continued by maturation of the centrosome into the basal body by acquisition of (sub)distal appendages (Figure $1 \mathrm{~A}$ and (54)). As a result, ciliogenesis can regulate cell cycle progression, because these are mutually exclusive processes, both requiring the centrosomes/basal body. FLCN has been implicated in cell cycle regulation. We show that FLCN localises at the centrosome during all stages of the cell cycle (Chapter 2 Figure 2 and Chapter 4 figure 2). In vivo, flcn is expressed in the proliferative zone of the brain of zebrafish embryos. Morpholino knockdown suggests that Flcn likely positively regulates cell cycle progression in certain tissues during zebrafish embryogenesis. In zFucci transgenic zebrafish flcn morphants, there is a decreased population of cells in S-M phase at 18 somite stage, particularly in the retina and the brain (Chapter 3), causing developmental defects. Laviolette et al. reported that loss of FLCN in vitro results in more rapid progression through the cell cycle, through late $\mathrm{S}$ and $\mathrm{G} 2 / \mathrm{M}$ phase (24). In addition, FLCN has been shown to negatively regulate expression of cyclin D1, a promoter of G1 to S phase progression $(51,55)$. It is therefore possible that the defects in ciliogenesis observed by us following FLCN knockdown are an indirect effect, resulting from stimulation of cell cycle progression. However, ciliary mutants which result in cilium depletion or reduction of axonemal length are prone to initiate rapid cell 
duplication when properly stimulated (47), suggesting that cell cycle defects could also be secondary to a ciliation issue in the FLCN knockdown cells.

\section{FLCN may influence ciliogenesis through regulation of small GTPases}

Yet another possible explanation for the delayed ciliogenesis is suggested by other studies of FLCN's interactions. After the centrosome has matured into the basal body, the next step of ciliogenesis is fusion of the basal body with a ciliary vesicle (Figure 1B). As the ciliary axoneme elongates from the basal body, the ciliary vesicle flattens into two layers of membrane, forming the ciliary sheath. This ciliary sheath grows by fusion with secondary ciliary vesicles (Figure 1C) (56). The complex then migrates and docks to the plasma membrane (Figure 1D and E and (56)). These events depend on the distribution of the cytoplasmic and apical actin cytoskeleton $(57,58)$. Actin cytoskeletal remodelling is regulated by activation of RhoA, a small GTPase belonging to the Ras superfamily, which has been linked to FLCN. FLCN has been shown to interact with p0071/plakophilin-4, a regulator of RhoA signalling $(59,60)$. FLCN deficiency resulted in deregulated RhoA signalling. Of interest, RhoA is a downstream effector of planar cell polarity genes (PCP), mediated by non-canonical Wnt signalling (61). In FLCN-deficient cells we observed an increase in the levels of unphosphorylated $\beta$-catenin (Chapter 2 Figure 6) suggesting increased activation of canonical Wnt signalling. This might inhibit non-canonical Wnt signalling (62) and consequently result in decreased RhoA activity, inhibiting cytoskeletal remodelling and ciliogenesis. This decreased PCP might also disrupt polarised cell division as observed in three-dimensional culture of renal cells with FLCN deficiency (Chapter 2 Figure 5).

After docking of the basal body to the plasmid membrane, growth of the ciliary axoneme is mediated by targeted vesicle transport (47). This is dependent on local actin filaments (63), also mediated by RhoA signalling, suggesting a possible role for FLCN in this process as well. In addition, this transport is mediated by small GTPases of the Rab and Arl/Raf subtypes (Figure 2) and kinesin molecular motor proteins that transport vesicles along microtubules linking the Golgi-ER to the basal body (47). The budding yeast FLCN ortholog, lethal with sec13 protein 7 (LST7), is a putative post-Golgi vesicle coat protein (64). FLCN also has a bipartite tryptophan-based kinesin light chain 1 interacting motif (WD-WQ) (65) which would support a role for FLCN in vesicular membrane trafficking. In addition, the crystal structure of human FLCN's carboxyl-terminus was presented recently, suggesting FLCN might act as a guanine exchange factor (GEF) for Rab35 (66), a small GTPase involved in endocytic recycling pathways (67). Although later studies could not confirm the Rab35 interaction (32), FLCN does bind various other Rab small G proteins involved in vesicular trafficking events linked to autophagy (E. Dunlop/A. Tee, personal communication 2014). In addition, FLCN has been localised to the surface of the lysosomes where it modulates amino acid stimulated mTORC1 recruitment $(31,32)$. This recruitment is sensitive to the guanine nucleotide bound state of the lysosome-enriched Rag GTPases. The nature of 
FLCN's involvement in this process is not quite clear. While Tsun et al. suggested that the FLCN-FNIP2 complex acts as a GTP-activating protein (GAP) for RagC and RagD (31), Petit et al. reported that FLCN preferentially binds the inactive forms of RagA/B and stimulates RagA activation, similar to GEFs (32). Although it may seem paradoxical, proteins with both GEF and GAP activity have been reported previously (68). Together, these data suggest that FLCN might regulate various GTPases of the Ras superfamily through GEF and/or GAP activity and thereby direct intracellular vesicular transport.

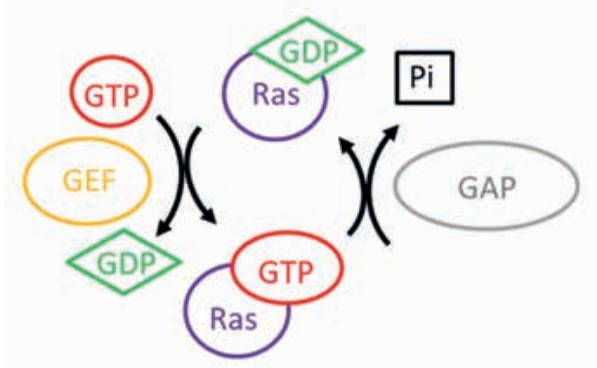

Figure 2 -Regulation of Ras small GTPases

GTP (red) bound to small GTPases of the Ras superfamily (including RhoA, Rab and Ran; purple) can be hydrolysed to GDP (green) by GTPase activating proteins (GAPs, grey). Guanine exchange factors (GEF; yellow) facilitate the release of GDP and the binding of GTP to Ras GTPases. Asymmetric distribution of GAPs and GEFs across various cellular compartments can cause concentration gradients of the GTP versus GDP bound form of the small GTPases.

FLCN could additionally influence ciliogenesis by controlling protein transport into the ciliary compartment via GTPases (Figure 2). The ciliary compartment is separated from the cytoplasm by a ciliary exclusion barrier formed by the transition zone and transition fibers (57). These structures provide a size limit for entry into the primary cilium. As there is no protein synthesis within the ciliary compartment, all proteins necessary for ciliary maintenance need to be actively transported in. This transport is dependent on a concentration gradient of the GTP versus GDP bound form of the small GTPase Ran (69), similar to nucleocytoplasmic transport (70). Analogous to the nucleus, high levels of RanGTP are present within the cilium and regulate KIF17 ciliary localisation $(69,71)$. FLCN could play a role in regulating this GTP versus GDP gradient in the both the cilium and the nucleus, through its putative GEF/GAP function.

\section{FLCN is likely not involved in intraflagellar transport}

Within the cilium proteins are directed via intraflagellar transport (IFT) along microtubules, involving kinesin-2 (anterograde transport) and dynein (retrograde transport) transport proteins. Regulation of ciliary length takes place by balancing tubulin assembly and disassembly at the distal end of the ciliary axoneme (72). Ciliary stability can also be altered via post translational modification of tubulin, via acetylation, detyrosination, polyglutamylation and polyglycylation (58). We show that in FLCN deficient cells there is a decreased percentage of ciliation and decreased ciliary length compared to controls after culture without serum for 48 hours. However, when serum deprivation is prolonged, the statistically significant differences disappear (Chapter 2 Figure 4). Therefore, it is tempting to speculate 
that FLCN is not involved in maintenance of cilia and IFT. Defects in IFT often lead to skeletal abnormalities (73), which are not seen in BHD. FLCN seems to be mainly involved in the early stages of ciliogenesis. Once ciliogenesis is underway, FLCN's role seems permissive.

\section{FLCN may control various cellular processes through regulation of small GTPase}

In conclusion, FLCN is highly conserved throughout evolution, with orthologs in Fungi and Animals, suggestive of a common role in the physiology of both Kingdoms. In Chapter 2 we show that FLCN regulates ciliogenesis, orientation of the mitotic spindle and canonical Wnt signalling. Malfunctioning or loss of primary cilia has been reported to drive cyst formation in various organs, including kidneys, liver and pancreas $(74,75)$. All organs affected in BHD patients, including hair follicles (76), are constructed of polarised epithelial cells generating cilia which protrude into a lumen. In these tissues primary cilia direct polarised cell division and control lumen formation (9), explaining how loss of FLCN leads to a cystic phenotype in BHD patients.

However, it still remains unclear how FLCN deficiency drives carcinogenesis. We suggest that FLCN is a major regulator of vesicle transport through a GAP and/or GEF function towards small GTPases of the Ras superfamily, including RhoA, Rab and Ran. This hypothesis would not only explain how FLCN affects ciliogenesis, through possible regulation of actin remodelling, vesicle transport and/or the ciliary RanGTP-RanGDP gradient, but also why FLCN deficiency has such widespread cellular consequences (Figure 3). Many processes affected by FLCN knockdown require proper vesicle transport and/or GTPase functioning. The basic-helix-loop-helix transcription factors $\operatorname{HIF} 1 \alpha(46,51)$, TFE3 $(77,78)$ and TFEB (32) all show increased nuclear localisation in cells with FLCN knockdown, suggesting nucleocytoplasmic transport regulated by Ran to be affected. RanGTP has also been suggested to play a role in assembly and orientation of the mitotic spindle, thereby influencing cell cycle progression (79). In addition, cell adhesion and polarity are established through localisation of specific proteins to particular areas of the cell membrane by targeted vesicle transport (80) and autophagy is a continuous vesicle transport process mediated by Rabs (81).

If FLCN's true function is indeed to direct intracellular vesicle transport, many of the pathways deregulated by loss of FLCN may represent indirect defects or compensatory mechanisms. This might explain why experimental outcome often seems to vary between various BHD animal models and cell lines, depending on tissue or species of origin of the cell type used, whether the cells are untransformed or have cancerous properties, the level of FLCN knockdown and/or the time-point of FLCN loss. 


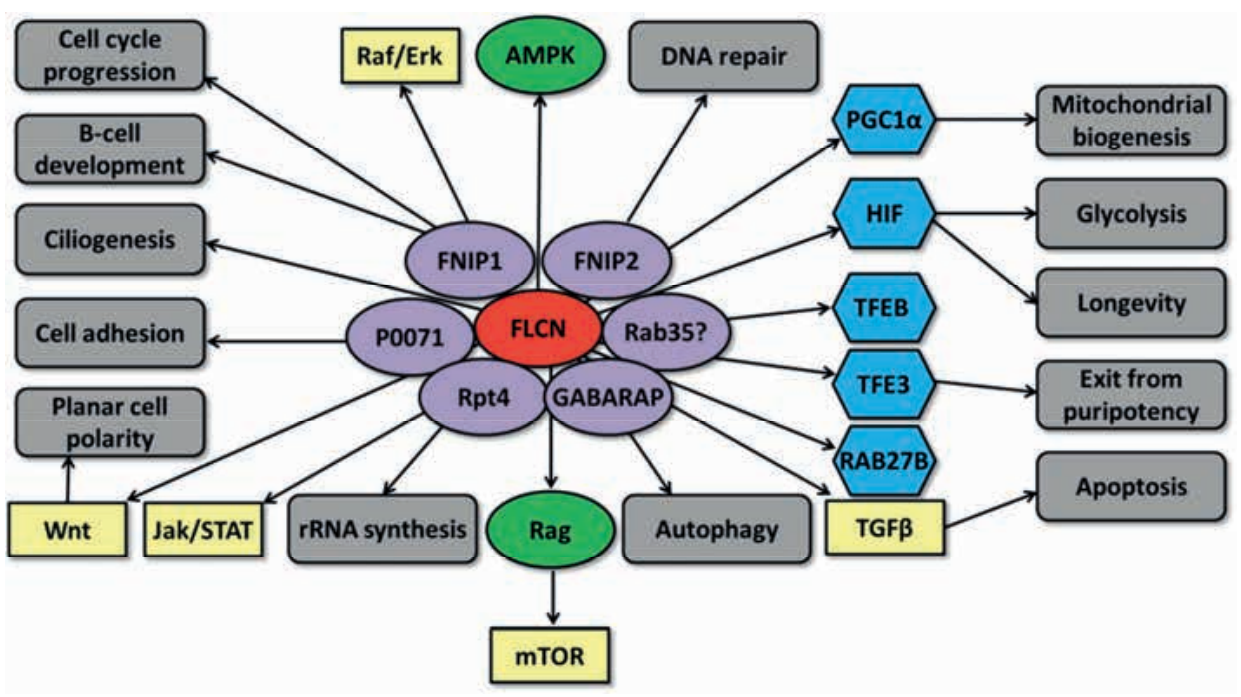

Figure 3 - FLCN signalling pathways

This figure summarises the state of current knowledge. FLCN (red) is embedded in a large number of signalling pathways. Known interacting proteins (purple) are depicted around FLCN. Pathways and targets regulated by FLCN are indicated in yellow, blue and green. Biological processes known to be affected by FLCN are indicated in grey.

\section{FNIP1 protein function}

In 2006, a novel protein was identified as an interactor of FLCN and named folliculin interacting protein 1 (FNIP1) (30). FNIP1 was shown to also interact with FNIP2 and AMPK (35), the latter being one of the major regulators of cellular energy homeostasis. AMPK is activated when the AMP:ATP ratio in the cells increases as a result of energy consumption (82). ATP-producing pathways such as autophagy and fatty acid oxidation are subsequently stimulated, while mTOR signalling is inhibited to reduce ATP-depleting processes (e.g. lipid and protein synthesis) (82). FNIP1 most likely operates downstream of AMPK, as AMPK phosphorylates FNIP1, thereby reducing FNIP1 protein levels (30). FNIP1 has also been linked to autophagy through interaction with GABARAP, an integral component of the autophagy machinery $(34,83)$. It was recently shown that Fnip1 is required for proper development of particular immune cell subtypes in mice. Park et al. report a developmental arrest of invariant natural killer T cells in Fnip1 knockout mice (84). In addition, two independent studies showed that homozygous knockout of Fnip1 in mice results in a deregulated B cell differentiation $(85,86)$. Park et al. detected a block in B cell development at the pre-B cell stage, with reduced mTOR inhibition after stimulation of AMPK with AICAR in the Fnip1-null pre-B cells (85). Phosphorylation of Akt, Raptor and AMPK itself were all normally regulated in the absence of Fnip1 (85), suggesting that Fnip1 is only required for AMPK-mediated inhibition of mTOR and not activation 
of energy production. In contrast, Baba et al. show that mTOR activity was not affected by the absence of Fnip1 (86). Conversely, they found a pro-B cell block, caused by caspase-induced cell death in pre-B cells (86), indicating that, similar to FLCN, the relationship between FNIP1 and mTOR may be complex and contextdependent.

In vitro, FNIP1 was shown to support the role of FLCN in various pathways. FNIP1 facilitates phosphorylation of FLCN and interaction with AMPK, Rag and GABARAP (30-34). Similar to FLCN deficiency, FNIP1 knockdown increased TFEB nuclear localisation (32). As FNIP1 knockdown may have an effect on FLCN levels, this might be an indirect effect. However, both FLCN (66) and FNIP1 (87) contain a DENN (differentially expressed in normal versus neoplastic cells) module and the expression patterns of flcn and fnip1 during zebrafish embryogenesis are highly similar (Chapter 3 and 4), suggesting that FLCN and FNIP1 have a similar function.

It is therefore surprising that humans with a FNIP1 mutation only have a relatively mild skin phenotype, described in Chapter 4 as familial multiple discoid fibromas (FMDF). We identify a heterozygous mutation in FNIP1 in FMDF patients and show that the mutant FNIP1 mRNA is broken down by nonsense-mediated decay. As we found no somatic second hits in primary fibroblasts derived from a discoid fibroma, we hypothesise that FMDF is caused by FNIP1 haploinsufficiency. Interestingly, in fibrofolliculomas, somatic second hits were detected in lesional fibroblasts (J. Toro, personal communication 2012), suggesting that these drive development of the BHD skin tumours upon loss of FLCN. This contrasts with FMDF, but the primary fibroblasts that we isolated from the discoid fibromas may not be a true representation of the lesions caused by FNIP1 mutation. To more definitively rule out a second hit/loss of heterozygosity, laser capture microdissection followed by sequencing could be performed.

Although the skin phenotype resulting from FNIP1 mutation is clinically similar to the BHD fibrofolliculomas, histologically the lesions are distinct. In addition, the discoid fibromas develop during childhood, in contrast to the BHD skin lesions which typically do not appear before the age of $25(20,42-44)$, which suggests they are caused by different mechanisms. How loss of FNIP1 results in the discoid fibromas remains to be determined as the exact function of FNIP1 is currently unknown. Although we detect FNIP1 at the primary cilium, similar to FLCN, FNIP1 deficiency in both HK-2 cells and primary fibroblasts does not result in changes to either ciliary number or appearance (Chapter 4 Figure S3). In addition, no obvious ciliary defects were detected in fnip1 morphant zebrafish embryos (Chapter 4 Figure S4). Thus, the patient phenotype is unlikely to reflect defective ciliogenesis, although defects in ciliary signalling cannot be ruled out.

Residues 602-929 comprise the minimum region of FNIP1 necessary for in vitro binding to AMPK (30). Even if small amounts of mutant protein would still be produced, the majority of these residues are deleted in FMDF patients, possibly disrupting AMPK signalling. Deregulation of AMPK and associated pathways has 


\section{CHAPTER 5}

been linked to uncontrolled growth and carcinogenesis $(82,88)$, possibly explaining the development of discoid fibromas after FNIP1 mutation.

As indicated, there is no evidence of systemic manifestations resulting from FNIP1 mutation. One possible explanation for this surprising finding is that there is tissue-specific compensation by FNIP2. FNIP1 and FNIP2 are paralogs and the latter seems to be more conserved throughout evolution, as the single Caenorhabditis elegans FNIP is more homologous to mammalian FNIP2 than to FNIP1. FNIP2 has higher expression levels in organs affected in BHD including lung, kidney, liver and pancreas $(35,36)$. Knockdown of FLCN in pluripotent stem cells resulted in decreased differentiation, which could only be mimicked by simultaneous knockdown of FNIP1 and FNIP2 (78), suggesting functional redundancy between the two. Alternatively, loss of a single FNIP1 allele might simply be tolerated in these tissues, possibly as a result of higher baseline FNIP1 levels compared to skin as determined by qPCR (35) and Western blot (Chapter 4).

In conclusion, we show that a heterozygous mutation in FNIP1, causing haploinsufficiency, leads to development of FMDF through an unknown cellular mechanism.

\section{Zebrafish models}

Although in vitro studies have provided clues about the function of FLCN and related proteins, this type of study has certain limitations. Various BHD research groups have generated contrasting results on the role of FLCN in certain signalling pathways, including RhoA signalling $(59,60)$, autophagy $(34,52)$ and regulation of Rag GTPases $(31,32)$. This may be partially explained by the fact that cultured cells are maintained in an artificial environment, which does not represent homeostatic or physiological conditions. Furthermore, extensive interactions between cells and tissues cannot be duplicated in in vitro models (89). Thus, we felt the need to study FLCN and FNIP1 in an animal model.

Various animal models for BHD, both naturally occurring and transgenic, have been characterised. These have shown that FLCN is required for embryonic development, as homozygous knockout of FLCN in vertebrates is embryonically lethal $(2,4,10-12,27,90)$. Mice with homozygous knockout of Flcn die before gastrulation (4), making it challenging to determine how Flcn influences development. Homozygous knockout of Fnip1 in mice is viable, resulting in an arrest of B cell and invariant natural killer T cell development (84-86). However, how this translates to the human FMDF lesions is currently unclear, suggesting that knockout mice may not be the best tools to study the function of FLCN and FNIP1.

Because of their ex vivo development enabling easy visualisation and manipulation, their large offspring production and similar tumour formation to humans, zebrafish (Danio rerio) are very well suited to study the pathophysiology of both developmental defects and carcinogenesis. In addition, many ciliopathies 
have been modelled in zebrafish (91-96) and these models show similar phenotypes. Cilia are easily visualised in zebrafish. Therefore, we decided to generate zebrafish loss of function models for Flcn and Fnip1 in order to learn more about the function of these proteins.

\section{Characterisation of Flcn and Fnip1 morphant zebrafish}

In Chapter 3 we characterise a zebrafish knockdown model for BHD and show that loss of zebrafish Flcn by injection of morpholino antisense oligonucleotides leads to a phenotype characterised by brain oedema, problems with tail circulation and a larger yolk and thinner yolk extension in 2-day old morphants. A zebrafish model for fnip1 knockdown using morpholinos suggests that Fnip1 is required for development, producing a phenotype similar to that of the flcn morphants (Chapter 4). Zebrafish flcn and fnip1 have similar expression patterns as determined by whole mount in situ hybridization. Both genes are expressed in the eye, fin bud, somites and regions of the brain, similar to the pattern of Flcn expression previously observed in the developing mouse embryo (4). We have determined that co-injection of flcn and fnip1 morpholinos does not aggravate the phenotype (unpublished), which is consistent with the suggestion that in zebrafish flch and fnip1 are epistatic.

Part of the flcn and fnip1 morphant phenotype is reminiscent of zebrafish morphants $(94,96,97)$ and mutants $(98)$ for ciliopathy genes. Zebrafish are very well suited for the study of cilia and the phenotypes associated with their disruption. Several embryonic zebrafish structures show formation of cilia, including the Kupffer's vesicle, the pronephric duct and the otic vesicle (99-101). Malfunctioning of the cilia in the Kupffer's vesicle has been reported to result in situs inversus, which is manifested early in embryonic development by randomised organisation of the heart and visceral organs $(92,101)$. Incidental cases of randomised laterality were observed in the flcn morphants, but the frequency was not high enough to detect statistically significant differences (unpublished). After extensive whole mount immunofluorescent studies using acetylated alpha tubulin antibodies in the flcn and fnip1 morphants no obvious defects were detected in the appearance of cilia in either pronephros or central canal (Chapter 3 and 4). However, the normal appearance does not rule out the possibility that the cilia that were detected in the morphants might not be functional. This could be investigated by monitoring fluid flow along the pronephros or the central canal after injection of rhodamine-conjugated dextran into the heart or the fourth ventricle, respectively $(91,92,102)$. Alternatively, there might be species-specific differences in FLCN's function in ciliogenesis.

In vitro, FLCN has been implicated in cell cycle regulation via late $\mathrm{S}$ and G2/M phase and cyclin D1 $(24,51,55)$. In addition, the flcn morphants show developmental arrest and flcn and fnip1 are expressed in the proliferative zone of the brain. Therefore, the lack of ciliary defects prompted us to examine whether the morphant phenotype was caused by cell cycle defects. Using the zFucci double 


\section{CHAPTER 5}

transgenic zebrafish line we show that flcn morphants have a decrease in the amount of cells in S, G2 and M phase and a corresponding increase in G1 cells, particularly in the retina and compartments of the brain including the tectum (Chapter 3 Figure 3 and 4). It remains to be determined if Flcn directly regulates the cell cycle in these embryos or whether these defects are secondary, possibly resulting from metabolic defect caused by Flcn deficiency.

In summary, zebrafish Flcn and Fnip1 loss of function models suggest that both proteins may be important for zebrafish embryogenesis, as their deficiency causes a severe developmental phenotype. Our data presently do not support ciliary dysfunction as a major contributing factor to the observed defects. Investigations in zFucci transgenic embryos suggest that these phenotypes may be caused by cell cycle defects.

\section{Characterisation of TILLING zebrafish flcn mutant}

The use of morpholinos has several limitations. It has become clear that managing off-target effects is an increasingly important issue in dealing with morpholino injections (103). As morpholinos induce p53-dependent side-effects $(104,105)$, co-injection with a morpholino directed against p53 is necessary to discriminate between genuine phenotypes and those resulting from p53-induced apoptosis (106). However, the definitive control for a morpholino knockdown is a mutant zebrafish line (105). Therefore, we aimed to characterise a zebrafish line with a flcn mutation.

Within three months, zebrafish larvae have developed into fertile adults (107), making generation of stable mutants or transgenic fish relatively quick compared to other animal models. To generate stable mutant fish, target-selected mutagenesis (TILLING) has been developed, which combines mutagenesis using $N$ ethyl- $N$-nitrosourea with DNA screening techniques to identify exonic mutations (108). In collaboration with the Sanger Centre a zebrafish strain was identified with a nonsense mutation in flcn, which results in a stop codon after 11 amino acids (Figure 4). Unexpectedly, both heterozygous and homozygous zebrafish showed normal embryonic development (unpublished). Heterozygous animals develop into adulthood without any obvious defects, while homozygous animals die around 1 month of age of a currently unknown cause. This contrasts with data from other available animal models for BHD, both naturally occurring $(2,27)$ and transgenic models $(4,10-12,90)$, which consistently show that homozygous knockout of Flcn is embryonically lethal. These observations are supported by our own flcn morphant data (Chapter 3).

The apparent discrepancy between the phenotypes of zebrafish flcn morphants versus mutants might due to effects of genetic background of the zebrafish. Many published articles report the use of "wild-type" zebrafish, which can refer to 
various strains. Currently, 29 different strains are listed as wild-type on the zebrafish model organism database ZFIN (https://www.zfin.org). Each of these is derived from a different founder stock, which is then maintained as a closed breeding population under selection pressure for traits suitable for laboratory purposes (e.g. removal of mutations leading to lethality). Therefore, separate strains may have a unique genetic background and when strains are maintained in separate laboratories over time genetic divergence occurs. Through epistatic gene action, genetic background has been reported to influence phenotypes expressed by various zebrafish morphants and mutants $(109,110)$. For example, Sanders and Whitlock showed that the severity of the developmental defects in zebrafish masterblind mutants decreased when the mutation was moved from the Tupfel long fin (TL) to the AB background (110). Strain-dependent effects on complex behaviours (111) and drug susceptibility $(112,113)$ have also be reported. The flcn morpholinos were injected into embryos from an $\mathrm{AB}$ background, while the flcn mutants come from the Tübingen (TU) background. To rule out any strain-specific effects that might cause a reduction in phenotype severity in the TU flcn mutants it would be interesting to inject the morpholinos into wild-type embryos from a TU background, which should reproduce the AB Flcn morphant phenotype if genetic background were not involved.

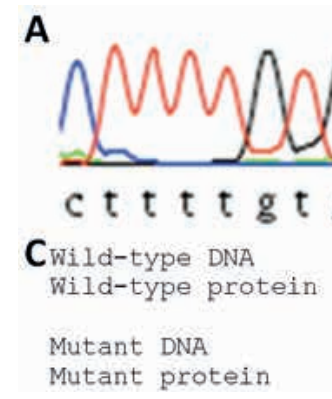

\section{B}

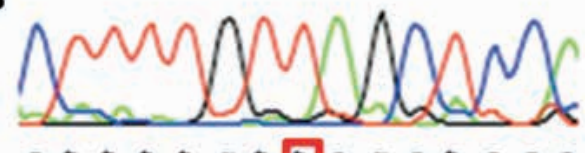
ct t t g t tagctc a

Figure 4 - Zebrafish flcn mutant

(A) Electropherogram of part of flcn genomic DNA in a wild-type Tübingen embryo. (B) Electropherogram of part of flcn genomic DNA showing homozygous mutation (red square) in Tübingen embryos with allele e36 from The Wellcome Trust Sanger Institute Zebrafish Mutation Project. (C) The c.34G $>\mathrm{T}$ mutation results in a stop codon after 11 amino acids.

Alternatively, this particular flcn mutation might not represent a true null allele. Several mechanisms have been reported that allow production of either fully or partially functional proteins from alleles with nonsense mutations (114). As efficiency of translation termination depends on the nucleotide sequence surrounding the stop codon, the premature stop codon may not be recognised as such, leading to continued protein production (115). Nonsense suppression is a well-known mechanism in eukaryotic organism such as yeast (115) and has been reported for proteins involved in human disease such as Menkes' disease and cystic fibrosis, influencing treatment outcome $(116,117)$. In the zebrafish flcn 


\section{CHAPTER 5}

mutants, nonsense suppression may lead to production of a low level of full-length Flcn. In addition, a truncated form of Flcn may be expressed when the exon containing the stop codon is removed from the mature mRNA, due to nonsenseassociated altered splicing (118). Also, for several disease-causing genes containing nonsense mutations, translation has been reported to be initiated from an internal start codon downstream of the premature stop codon $(114,119)$. The flcn gene contains six possible start codons downstream of the nonsense codon, of which an ATG in exon 2 meets the Kozak sequence requirements for efficient initiation of translation (120). Translation starting from this alternative start codon would lead to expression of a truncated protein lacking $163 \mathrm{~N}$-terminal amino acids. As the majority of the disease-causing mutations in BHD result in deletion of the C-terminus (42), it is conceivable that the protein translated from this alternative start codon may be largely functional.

Thus, low levels of full length or truncated Flcn could be expressed via (one of) these mechanisms in the flcn mutant embryos, which might result in partial rescue of the phenotype induced by loss of Flcn. Injection of morpholinos against these alternative forms of Flcn in the mutant embryos should then reproduce the morphant phenotype. Detection of endogenous Flcn in protein extract from wildtype zebrafish embryos has proven challenging using available antibodies, which are all raised against human FLCN peptide (Figure 5). Therefore, a custom made rabbit antibody against a peptide consisting of amino acids GGSNPQSSQSESVQA of zebrafish Flcn was generated and affinity purified by Eurogentec (Seraing, Belgium). Although this antibody specifically recognised zebrafish Flcn peptide expressed from bacterial and mammalian vectors, Western blots performed for detection of endogenous Flcn have not generated conclusive results (Figure 5). Alternatively, translation from alternative start codons can also be confirmed in vitro, using a series of flcn fragments with the putative alternative initiation codons intact or mutated to other amino acid coding sequences. After in vitro transcription and translation or after transfection into cells, Western blot could be used to determine which of these start codons can be used for protein production.

Lastly, the discrepancy between the phenotypes of zebrafish flcn morphants versus mutants might be caused by additional off-target effects induced by the morpholino. Development of novel techniques to manipulate the zebrafish genome provide strong indications that morphants phenotypes might not be a true representation of gene function, as outlined below.

\section{Zebrafish with mutations at targeted sites}

As TILLING does not allow control over the site of mutagenesis, alternative methods have been developed to induce specific mutations at targeted sites, using zinc finger endonucleases (ZFNs) and TALENs (121). These proteins are fusions of the DNA-cleaving domain of the endonuclease FokI either to a DNA-binding zinc finger protein or to a transcription activator-like effector (TALE). Such proteins bind genomic DNA at user-specified locations, followed by the induction of double- 
strand DNA breaks by the endonuclease. The breaks are then imprecisely repaired by non-homologous end joining, leading to introduction of small insertions or deletions at the target site (121).
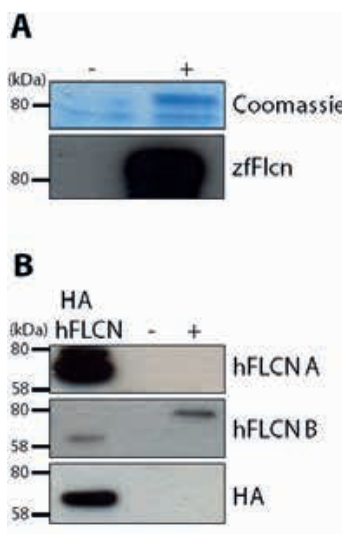

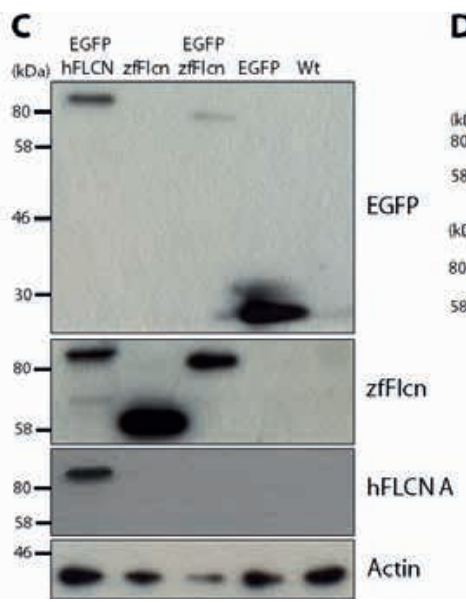

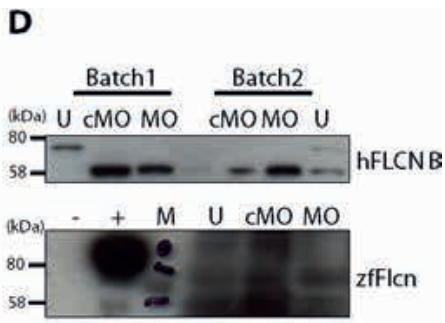

Figure 5 -Western blot test for detection of zebrafish Flcn

(A) A custom-made antibody against zebrafish Flcn peptide binds GST-6xHis-tagged zebrafish Flcn (89 $\mathrm{kDa}$ ) expressed by bacteria from pET41b-zfFlcn after induction (+)(lower panel). Production of GST6xHis-tagged zebrafish Flcn $(89 \mathrm{kDa})$ confirmed in induced $(+)$ but not uninduced $(-)$ bacteria using Coomassie (upper panel). (B) Both antibodies A and B against human FLCN bind HA-tagged human FLCN (63kDa) expressed in HEK293 cells. Only antibody B binds GST-6xHis-tagged zebrafish Flcn (89 $\mathrm{kDa}$ ) expressed by bacteria from pET41b-zfFlcn after induction (+). Extract from uninduced bacteria (-) was used as a negative control. (C) Custom-made antibody against zebrafish Flcn peptide binds EGFPtagged human FLCN (94 kDa), EGFP-tagged zebrafish Flcn (89 kDa) and zebrafish Flcn (62 kDa) expressed in HEK293 cells. Antibody A against human FLCN only binds EGFP-tagged human FLCN. Extract from wild-type HEK293 cells (Wt) or cells expressing or EGFP (23 kDa) was used as a control.

(D) Both antibodies that bind overexpressed zebrafish Flcn protein in panel A-C give inconclusive results on protein extract from 48 hpf uninjected zebrafish embryos (U) or embryos injected with flcn splice 2 morpholino (MO) or five basepair mismatch morpholino (cMO). Extract from bacteria transformed with pET41b-zfFlcn either uninduced (-) or induced (+) to express GST-6xHis-tagged zebrafish Flcn $(89 \mathrm{kDa})$ was used as a control. The third lane contains marker (M).

Recently a novel, potentially very powerful tool has emerged for genome editing: the clustered regularly interspaced short palindromic repeats (CRISPR) - CRISPRassociated proteins (Cas) system. Originally identified as part of the bacterial immune system against invading viruses and plasmids, the type II CRISPR-Cas system depends on short CRISPR RNA segments (crRNA) which anneal to transactivating crRNAs (tracrRNA), directing sequence-specific cleavage and silencing of pathogenic DNA by Cas proteins (122). Jinek et al. have been able to generate synthetic single guide RNA (sgRNA) consisting of a fusion between crRNA and tracrRNA to direct cleavage of target DNA by the endonuclease Cas9 (28). The CRISPR/Cas 9 system has successfully been used in zebrafish to generate heritable mutations and transgenic lines, with high mutagenesis and integration rates (123125). In comparison to ZFN and TALENs, which require assembly of two nucleases 


\section{CHAPTER 5}

for each target site, the CRISPR/Cas system is more straightforward as only the sgRNA of approximately 20 nucleotides needs to be designed and constructed.

Surprisingly, $80 \%$ out of 80 cases of CRISPR/Cas9 mutant zebrafish lines for which a morphant was also available did not recapitulate the morpholino-induced phenotype, showing milder defects or even complete absence of developmental defects (N. Lawson, personal communication 2014). These aggravated phenotypes in the morphants can be rescued by co-injection of morpholino and capped mRNA of the gene of interest. Therefore, they are most likely not solely due to morpholino injection alone. If this were the case, one would also expect the mismatch morpholinos to generate developmental defects and there is no notice of this in literature. Although publication bias could play a role, because scientists are likely to select a mismatch morpholino that does not give a phenotype, this may not explain the full $80 \%$ discrepancy between the morphant and mutant zebrafish.

Together these data suggest that the aggravated morphant phenotype might have gene-specific components. Possibly, the microinjection generates a stress response in the zebrafish embryos, thereby worsening the effect of gene deficiency. These defects would then not represent genuine off-target effects merely from morpholino injection, but a combination of specific targeting events and a nonspecific stress response to the morpholino injection.

To ensure specificity of the flcn and fnip1 morphant phenotypes it would be of interest to generate mutant zebrafish lines using the techniques outlined above. As the BHD protein complex consists of FLCN, FNIP1 and FNIP2, generating a fnip2 zebrafish mutant could also provide insight into the pathogenesis of BHD and related disorders. Currently, the only animal model available to study the function of FNIP2 is the Weimaraner dog, in which homozygosity for the FNIP2 mutation c.880delA (p.I294fsX296) leads to hypomyelination of the brain and spinal cord (126).

These zebrafish mutants would make it possible to investigate the long-term effects of Flcn/Fnip1/Fnip2 deficiency in adult zebrafish. BHD patients are predisposed to the development of renal cancer, but morpholinos are active only for approximately three days, too short a time frame for cancer to develop. In addition, BHD and FMDF patients are not FLCN- or FNIP1-null, but develop through embryogenesis as heterozygotes. Using these novel techniques it is possible to generate zebrafish mutant lines with flcn or fnip1 mutations at targeted locations, better resembling the human situation. BHD is a rare disorder; approximately 200 families have been diagnosed worldwide. As a result of the small number of patients and the large variety of FLCN mutations, genotypephenotype relations have been proven difficult to determine. Due to large offspring production zebrafish might provide a tool to investigate these relationships, by generation of an allelic series, that is a series of multiple mutant lines with 
different targeted flcn mutations (7). As past studies only incidentally investigated one or two variants of mutant FLCN, such a series could provide more conclusive results about the functionality of mutant Flcn versions, as true null alleles should produce the strongest phenotypes (7). Additionally, rescue experiments with mRNA encoding various mutant forms of Flcn could be used to systematically examine the hypothesis that mutant proteins are not functional. The large offspring production combined with the ease of scoring embryonic phenotypes and the simplicity of adding drugs makes implementation of large drug screens in zebrafish available (127). Similar to screens described for Dravet syndrome (128) and Duchenne muscular dystrophy (129), BHD zebrafish models could be screened for compounds that can relieve the mutant phenotype thus potentially benefitting BHD patients.

Generation of zebrafish mutants for flcn, fnip1 and fnip2 will provide valuable insight into the function of the proteins they encode and will help clarify the pathogenesis of associated disease.

\section{General conclusion}

Although its exact function is not completely understood, it has become apparent that FLCN is vital for cellular physiology. FLCN deficiency has widespread cellular consequences (Figure 3), including defective ciliogenesis and deregulated canonical Wnt signalling, which could explain why BHD patients develop cysts. However, it remains to be determined how loss of FLCN leads to cancer. During the past ten years, a growing body of data has been published on the putative function of FLCN, collectively suggesting a role for the protein in vesicular transport via regulation of small GTPases. Further research is required to confirm this hypothesis and unravel the cellular role of FLCN and its interactors, including FNIP1. This may lead to identification of novel therapeutic targets. As the exact function of FLCN and FNIP1 is not known, treatment for BHD and FMDF patients is currently empirical. Development of mutant zebrafish lines for flcn, fnip1 and fnip2 may prove valuable tools for drug screening, identifying compounds potentially benefitting BHD and FMDF patients even if complete understanding of the function of FLCN and FNIP1 is lacking. In addition, the clinical manifestations of BHD such as renal cell carcinoma and cyst formation in organs including lung, liver and kidney are common in the general population (130-133). Many of the pathways affected in BHD are also deregulated in sporadic renal cell carcinoma. Therefore, investigation of BHD pathophysiology may provide a unique opportunity to study the aetiology of these more common disorders. As FLCN is an ancient and crucial component of the cellular machinery, elucidating its functions will provide biological insights that are certain to profoundly affect other fields of inquiry. 


\section{CHAPTER 5}

\section{References}

1. Vocke CD, Yang Y, Pavlovich CP, Schmidt LS, Nickerson ML, Torres-Cabala CA, et al. High frequency of somatic frameshift BHD gene mutations in Birt-Hogg-Dubeassociated renal tumors. Journal of the National Cancer Institute. 2005 Jun 15;97(12):931-5. PubMed PMID: 15956655.

2. Okimoto K, Sakurai J, Kobayashi T, Mitani H, Hirayama Y, Nickerson ML, et al. A germline insertion in the Birt-Hogg-Dube (BHD) gene gives rise to the Nihon rat model of inherited renal cancer. Proceedings of the National Academy of Sciences of the United States of America. 2004 Feb 17;101(7):2023-7. PubMed PMID: 14769940. Pubmed Central PMCID: 357045.

3. Bonsdorff TB, Jansen JH, Lingaas F. Second hits in the FLCN gene in a hereditary renal cancer syndrome in dogs. Mammalian genome : official journal of the International Mammalian Genome Society. 2008 Feb;19(2):121-6. PubMed PMID: 18219524.

4. Hasumi Y, Baba M, Ajima R, Hasumi H, Valera VA, Klein ME, et al. Homozygous loss of BHD causes early embryonic lethality and kidney tumor development with activation of mTORC1 and mTORC2. Proceedings of the National Academy of Sciences of the United States of America. 2009 Nov 3;106(44):18722-7. PubMed PMID: 19850877. Pubmed Central PMCID: 2765925.

5. Knudson AG, Jr. Mutation and cancer: statistical study of retinoblastoma. Proceedings of the National Academy of Sciences of the United States of America. 1971 Apr;68(4):820-3. PubMed PMID: 5279523. Pubmed Central PMCID: 389051.

6. Hong SB, Oh H, Valera VA, Stull J, Ngo DT, Baba M, et al. Tumor suppressor FLCN inhibits tumorigenesis of a FLCN-null renal cancer cell line and regulates expression of key molecules in TGF-beta signaling. Molecular cancer. 2010;9:160. PubMed PMID: 20573232. Pubmed Central PMCID: 2907329.

7. Payne SR, Kemp CJ. Tumor suppressor genetics. Carcinogenesis. 2005 Dec;26(12):2031-45. PubMed PMID: 16150895.

8. van Steensel MA, Verstraeten VL, Frank J, Kelleners-Smeets NW, Poblete-Gutierrez P, Marcus-Soekarman D, et al. Novel mutations in the BHD gene and absence of loss of heterozygosity in fibrofolliculomas of Birt-Hogg-Dube patients. The Journal of investigative dermatology. 2007 Mar;127(3):588-93. PubMed PMID: 17124507.

9. McNeill H. Planar cell polarity and the kidney. Journal of the American Society of Nephrology : JASN. 2009 Oct;20(10):2104-11. PubMed PMID: 19762494.

10. Hartman TR, Nicolas E, Klein-Szanto A, Al-Saleem T, Cash TP, Simon MC, et al. The role of the Birt-Hogg-Dube protein in mTOR activation and renal tumorigenesis. Oncogene. 2009 Apr 2;28(13):1594-604. PubMed PMID: 19234517. Pubmed Central PMCID: 2664853.

11. Chen J, Futami K, Petillo D, Peng J, Wang P, Knol J, et al. Deficiency of FLCN in mouse kidney led to development of polycystic kidneys and renal neoplasia. PloS one. 2008;3(10):e3581. PubMed PMID: 18974783. Pubmed Central PMCID: 2570491.

12. Baba M, Furihata M, Hong SB, Tessarollo L, Haines DC, Southon E, et al. Kidneytargeted Birt-Hogg-Dube gene inactivation in a mouse model: Erk1/2 and Akt-mTOR activation, cell hyperproliferation, and polycystic kidneys. Journal of the National Cancer Institute. 2008 Jan 16;100(2):140-54. PubMed PMID: 18182616. Pubmed Central PMCID: 2704336.

13. Vlangos CN, Yim DK, Elsea SH. Refinement of the Smith-Magenis syndrome critical region to approximately $950 \mathrm{~kb}$ and assessment of $17 \mathrm{p} 11.2$ deletions. Are all deletions created equally? Molecular genetics and metabolism. 2003 Jun;79(2):134-41. PubMed PMID: 12809645. 
14. Slager RE, Newton TL, Vlangos CN, Finucane B, Elsea SH. Mutations in RAI1 associated with Smith-Magenis syndrome. Nature genetics. 2003 Apr;33(4):466-8. PubMed PMID: 12652298.

15. Greenberg F, Lewis RA, Potocki L, Glaze D, Parke J, Killian J, et al. Multi-disciplinary clinical study of Smith-Magenis syndrome (deletion 17p11.2). American journal of medical genetics. 1996 Mar 29;62(3):247-54. PubMed PMID: 8882782.

16. Edelman EA, Girirajan S, Finucane B, Patel PI, Lupski JR, Smith AC, et al. Gender, genotype, and phenotype differences in Smith-Magenis syndrome: a meta-analysis of 105 cases. Clinical genetics. 2007 Jun;71(6):540-50. PubMed PMID: 17539903.

17. Menko FH, Johannesma PC, van Moorselaar RJ, Reinhard R, van Waesberghe JH, Thunnissen E, et al. A de novo FLCN mutation in a patient with spontaneous pneumothorax and renal cancer; a clinical and molecular evaluation. Familial cancer. 2013 Sep;12(3):373-9. PubMed PMID: 23264078.

18. Strachan P, Read AP. Human Molecular Genetics. : New York: Wiley-Liss; 1999.

19. Bessis D, Giraud S, Richard S. A novel familial germline mutation in the initiator codon of the BHD gene in a patient with Birt-Hogg-Dube syndrome. The British journal of dermatology. 2006 Nov;155(5):1067-9. PubMed PMID: 17034545.

20. Kluger N, Giraud S, Coupier I, Avril MF, Dereure O, Guillot B, et al. Birt-Hogg-Dube syndrome: clinical and genetic studies of 10 French families. The British journal of dermatology. 2010 Mar;162(3):527-37. PubMed PMID: 19785621.

21. Bazykin GA, Kochetov AV. Alternative translation start sites are conserved in eukaryotic genomes. Nucleic acids research. 2011 Jan;39(2):567-77. PubMed PMID: 20864444. Pubmed Central PMCID: 3025576.

22. Benhammou JN, Vocke CD, Santani A, Schmidt LS, Baba M, Seyama K, et al. Identification of intragenic deletions and duplication in the FLCN gene in Birt-HoggDube syndrome. Genes, chromosomes \& cancer. 2011 Jun;50(6):466-77. PubMed PMID: 21412933. Pubmed Central PMCID: 3075348.

23. Nahorski MS, Reiman A, Lim DH, Nookala RK, Seabra L, Lu X, et al. Birt Hogg-Dube syndrome-associated FLCN mutations disrupt protein stability. Human mutation. 2011 Aug;32(8):921-9. PubMed PMID: 21538689.

24. Laviolette LA, Wilson J, Koller J, Neil C, Hulick P, Rejtar T, et al. Human folliculin delays cell cycle progression through late S and G2/M-phases: effect of phosphorylation and tumor associated mutations. PloS one. 2013;8(7):e66775. PubMed PMID: 23874397. Pubmed Central PMCID: 3708955.

25. Warren MB, Torres-Cabala CA, Turner ML, Merino MJ, Matrosova VY, Nickerson ML, et al. Expression of Birt-Hogg-Dube gene mRNA in normal and neoplastic human tissues. Modern pathology : an official journal of the United States and Canadian Academy of Pathology, Inc. 2004 Aug;17(8):998-1011. PubMed PMID: 15143337.

26. Yang Y, Padilla-Nash HM, Vira MA, Abu-Asab MS, Val D, Worrell R, et al. The UOK 257 cell line: a novel model for studies of the human Birt-Hogg-Dube gene pathway. Cancer genetics and cytogenetics. 2008 Jan 15;180(2):100-9. PubMed PMID: 18206534. Pubmed Central PMCID: 2440670.

27. Lingaas F, Comstock KE, Kirkness EF, Sorensen A, Aarskaug T, Hitte C, et al. A mutation in the canine BHD gene is associated with hereditary multifocal renal cystadenocarcinoma and nodular dermatofibrosis in the German Shepherd dog. Human molecular genetics. 2003 Dec 1;12(23):3043-53. PubMed PMID: 14532326.

28. Jinek M, Chylinski K, Fonfara I, Hauer M, Doudna JA, Charpentier E. A programmable dual-RNA-guided DNA endonuclease in adaptive bacterial immunity. Science. 2012 Aug 17;337(6096):816-21. PubMed PMID: 22745249.

29. Wong SP, Harbottle RP. Genetic modification of dividing cells using episomally maintained S/MAR DNA vectors. Molecular therapy Nucleic acids. 2013;2:e115. PubMed PMID: 23941867. Pubmed Central PMCID: 3759738. 
30. Baba M, Hong SB, Sharma N, Warren MB, Nickerson ML, Iwamatsu A, et al. Folliculin encoded by the BHD gene interacts with a binding protein, FNIP1, and AMPK, and is involved in AMPK and mTOR signaling. Proceedings of the National Academy of Sciences of the United States of America. 2006 Oct 17;103(42):15552-7. PubMed PMID: 17028174. Pubmed Central PMCID: 1592464.

31. Tsun ZY, Bar-Peled L, Chantranupong L, Zoncu R, Wang T, Kim C, et al. The folliculin tumor suppressor is a GAP for the RagC/D GTPases that signal amino acid levels to mTORC1. Molecular cell. 2013 Nov 21;52(4):495-505. PubMed PMID: 24095279. Pubmed Central PMCID: 3867817.

32. Petit CS, Roczniak-Ferguson A, Ferguson SM. Recruitment of folliculin to lysosomes supports the amino acid-dependent activation of Rag GTPases. The Journal of cell biology. 2013 Sep 30;202(7):1107-22. PubMed PMID: 24081491. Pubmed Central PMCID: 3787382.

33. Wang L, Kobayashi T, Piao X, Shiono M, Takagi Y, Mineki R, et al. Serine 62 is a phosphorylation site in folliculin, the Birt-Hogg-Dube gene product. FEBS letters. 2010 Jan 4;584(1):39-43. PubMed PMID: 19914239.

34. Dunlop EA, Seifan S, Claessens T, Behrends C, Kamps M, Rozycka E, et al. FLCN, a novel autophagy component, interacts with GABARAP and is regulated by ULK1 phosphorylation. Autophagy. 2014;0:0 - -1.

35. Hasumi H, Baba M, Hong SB, Hasumi Y, Huang Y, Yao M, et al. Identification and characterization of a novel folliculin-interacting protein FNIP2. Gene. 2008 May 31;415(1-2):60-7. PubMed PMID: 18403135. Pubmed Central PMCID: 2727720.

36. Takagi Y, Kobayashi T, Shiono M, Wang L, Piao X, Sun G, et al. Interaction of folliculin (Birt-Hogg-Dube gene product) with a novel Fnip1-like (FnipL/Fnip2) protein. Oncogene. 2008 Sep 11;27(40):5339-47. PubMed PMID: 18663353.

37. Tyburczy ME, Wang JA, Li S, Thangapazham R, Chekaluk Y, Moss J, et al. Sun exposure causes somatic second-hit mutations and angiofibroma development in tuberous sclerosis complex. Human molecular genetics. 2014 Apr 15;23(8):2023-9. PubMed PMID: 24271014. Pubmed Central PMCID: 3959815.

38. Lubensky IA, Gnarra JR, Bertheau P, Walther MM, Linehan WM, Zhuang Z. Allelic deletions of the VHL gene detected in multiple microscopic clear cell renal lesions in von Hippel-Lindau disease patients. The American journal of pathology. 1996 Dec;149(6):2089-94. PubMed PMID: 8952541. Pubmed Central PMCID: 1865348.

39. Mohr VH, Vortmeyer AO, Zhuang Z, Libutti SK, Walther MM, Choyke PL, et al. Histopathology and molecular genetics of multiple cysts and microcystic (serous) adenomas of the pancreas in von Hippel-Lindau patients. The American journal of pathology. 2000 Nov;157(5):1615-21. PubMed PMID: 11073821. Pubmed Central PMCID: 1885721.

40. Montani M, Heinimann K, von Teichman A, Rudolph T, Perren A, Moch H. VHL-gene deletion in single renal tubular epithelial cells and renal tubular cysts: further evidence for a cyst-dependent progression pathway of clear cell renal carcinoma in von Hippel-Lindau disease. The American journal of surgical pathology. 2010 Jun;34(6):806-15. PubMed PMID: 20431476.

41. Truong LD, Choi YJ, Shen SS, Ayala G, Amato R, Krishnan B. Renal cystic neoplasms and renal neoplasms associated with cystic renal diseases: pathogenetic and molecular links. Advances in anatomic pathology. 2003 May;10(3):135-59. PubMed PMID: 12717117.

42. Schmidt LS, Nickerson ML, Warren MB, Glenn GM, Toro JR, Merino MJ, et al. Germline BHD-mutation spectrum and phenotype analysis of a large cohort of families with BirtHogg-Dube syndrome. American journal of human genetics. 2005 Jun;76(6):1023-33. PubMed PMID: 15852235. Pubmed Central PMCID: 1196440. 
43. Toro JR, Wei MH, Glenn GM, Weinreich M, Toure O, Vocke C, et al. BHD mutations, clinical and molecular genetic investigations of Birt-Hogg-Dube syndrome: a new series of 50 families and a review of published reports. Journal of medical genetics. 2008 Jun;45(6):321-31. PubMed PMID: 18234728. Pubmed Central PMCID: 2564862.

44. Houweling AC, Gijezen LM, Jonker MA, van Doorn MB, Oldenburg RA, van SpaendonckZwarts KY, et al. Renal cancer and pneumothorax risk in Birt-Hogg-Dube syndrome; an analysis of 115 FLCN mutation carriers from 35 BHD families. British journal of cancer. 2011 Dec 6;105(12):1912-9. PubMed PMID: 22146830. Pubmed Central PMCID: 3251884.

45. Possik E, Jalali Z, Nouet Y, Yan M, Gingras MC, Schmeisser K, et al. Folliculin regulates ampk-dependent autophagy and metabolic stress survival. PLoS genetics. 2014 Apr;10(4):e1004273. PubMed PMID: 24763318.

46. Yan M, Gingras MC, Dunlop EA, Nouet Y, Dupuy F, Jalali Z, et al. The tumor suppressor folliculin regulates AMPK-dependent metabolic transformation. The Journal of clinical investigation. 2014 Apr 24. PubMed PMID: 24762438.

47. Basten SG, Giles RH. Functional aspects of primary cilia in signaling, cell cycle and tumorigenesis. Cilia. 2013;2(1):6. PubMed PMID: 23628112. Pubmed Central PMCID: 3662159.

48. Schmidt LS. Birt-Hogg-Dube syndrome: from gene discovery to molecularly targeted therapies. Familial cancer. 2013 Sep;12(3):357-64. PubMed PMID: 23108783. Pubmed Central PMCID: 3637987.

49. Tee AR, Pause A. Birt-Hogg-Dube: tumour suppressor function and signalling dynamics central to folliculin. Familial cancer. 2013 Sep;12(3):367-72. PubMed PMID: 23096221.

50. Laplante M, Sabatini DM. mTOR signaling at a glance. Journal of cell science. 2009 Oct 15;122(Pt 20):3589-94. PubMed PMID: 19812304. Pubmed Central PMCID: 2758797.

51. Preston RS, Philp A, Claessens T, Gijezen L, Dydensborg AB, Dunlop EA, et al. Absence of the Birt-Hogg-Dube gene product is associated with increased hypoxia-inducible factor transcriptional activity and a loss of metabolic flexibility. Oncogene. 2011 Mar 10;30(10):1159-73. PubMed PMID: 21057536. Pubmed Central PMCID: 3787473.

52. Tang Z, Lin MG, Stowe TR, Chen S, Zhu M, Stearns T, et al. Autophagy promotes primary ciliogenesis by removing OFD1 from centriolar satellites. Nature. 2013 Oct 10;502(7470):254-7. PubMed PMID: 24089205.

53. Pampliega O, Orhon I, Patel B, Sridhar S, Diaz-Carretero A, Beau I, et al. Functional interaction between autophagy and ciliogenesis. Nature. 2013 Oct 10;502(7470):194200. PubMed PMID: 24089209. Pubmed Central PMCID: 3896125.

54. Kobayashi T, Dynlacht BD. Regulating the transition from centriole to basal body. The Journal of cell biology. 2011 May 2;193(3):435-44. PubMed PMID: 21536747. Pubmed Central PMCID: 3087006.

55. Kawai A, Kobayashi T, Hino O. Folliculin regulates cyclin D1 expression through cisacting elements in the 3' untranslated region of cyclin D1 mRNA. International journal of oncology. 2013 May;42(5):1597-604. PubMed PMID: 23525507.

56. Sorokin S. Centrioles and the formation of rudimentary cilia by fibroblasts and smooth muscle cells. The Journal of cell biology. 1962 Nov;15:363-77. PubMed PMID: 13978319. Pubmed Central PMCID: 2106144.

57. Reiter JF, Blacque OE, Leroux MR. The base of the cilium: roles for transition fibres and the transition zone in ciliary formation, maintenance and compartmentalization. EMBO reports. 2012 Jul;13(7):608-18. PubMed PMID: 22653444. Pubmed Central PMCID: 3388784.

58. Avasthi P, Marshall WF. Stages of ciliogenesis and regulation of ciliary length. Differentiation; research in biological diversity. 2012 Feb;83(2):S30-42. PubMed PMID: 22178116. Pubmed Central PMCID: 3269565. 


\section{CHAPTER 5}

59. Medvetz DA, Khabibullin D, Hariharan V, Ongusaha PP, Goncharova EA, Schlechter T, et al. Folliculin, the product of the Birt-Hogg-Dube tumor suppressor gene, interacts with the adherens junction protein p0071 to regulate cell-cell adhesion. PloS one. 2012;7(11):e47842. PubMed PMID: 23139756. Pubmed Central PMCID: 3490959.

60. Nahorski MS, Seabra L, Straatman-Iwanowska A, Wingenfeld A, Reiman A, Lu X, et al. Folliculin interacts with p0071 (plakophilin-4) and deficiency is associated with disordered RhoA signalling, epithelial polarization and cytokinesis. Human molecular genetics. 2012 Dec 15;21(24):5268-79. PubMed PMID: 22965878. Pubmed Central PMCID: 3755511.

61. Komiya Y, Habas R. Wnt signal transduction pathways. Organogenesis. 2008 Apr;4(2):68-75. PubMed PMID: 19279717. Pubmed Central PMCID: 2634250.

62. Wallingford JB, Mitchell B. Strange as it may seem: the many links between Wnt signaling, planar cell polarity, and cilia. Genes \& development. 2011 Feb 1;25(3):20113. PubMed PMID: 21289065. Pubmed Central PMCID: 3034894.

63. Kim J, Lee JE, Heynen-Genel S, Suyama E, Ono K, Lee K, et al. Functional genomic screen for modulators of ciliogenesis and cilium length. Nature. 2010 Apr 15;464(7291):1048-51. PubMed PMID: 20393563. Pubmed Central PMCID: 2929961.

64. Roberg KJ, Bickel S, Rowley N, Kaiser CA. Control of amino acid permease sorting in the late secretory pathway of Saccharomyces cerevisiae by SEC13, LST4, LST7 and LST8. Genetics. 1997 Dec;147(4):1569-84. PubMed PMID: 9409822. Pubmed Central PMCID: 1208332.

65. Dodding MP, Mitter R, Humphries AC, Way M. A kinesin-1 binding motif in vaccinia virus that is widespread throughout the human genome. The EMBO journal. $2011 \mathrm{Nov}$ 16;30(22):4523-38. PubMed PMID: 21915095. Pubmed Central PMCID: 3243594.

66. Nookala RK, Langemeyer L, Pacitto A, Ochoa-Montano B, Donaldson JC, Blaszczyk BK, et al. Crystal structure of folliculin reveals a hidDENN function in genetically inherited renal cancer. Open biology. 2012 Aug;2(8):120071. PubMed PMID: 22977732. Pubmed Central PMCID: 3438538.

67. Chesneau L, Dambournet D, Machicoane M, Kouranti I, Fukuda M, Goud B, et al. An ARF6/Rab35 GTPase cascade for endocytic recycling and successful cytokinesis. Current biology : CB. 2012 Jan 24;22(2):147-53. PubMed PMID: 22226746.

68. Cho YJ, Cunnick JM, Yi SJ, Kaartinen V, Groffen J, Heisterkamp N. Abr and Bcr, two homologous Rac GTPase-activating proteins, control multiple cellular functions of murine macrophages. Molecular and cellular biology. 2007 Feb;27(3):899-911. PubMed PMID: 17116687. Pubmed Central PMCID: 1800684.

69. Dishinger JF, Kee HL, Jenkins PM, Fan S, Hurd TW, Hammond JW, et al. Ciliary entry of the kinesin-2 motor KIF17 is regulated by importin-beta2 and RanGTP. Nature cell biology. 2010 Jul;12(7):703-10. PubMed PMID: 20526328. Pubmed Central PMCID: 2896429.

70. Cook A, Bono F, Jinek M, Conti E. Structural biology of nucleocytoplasmic transport. Annual review of biochemistry. 2007;76:647-71. PubMed PMID: 17506639.

71. Fan S, Whiteman EL, Hurd TW, McIntyre JC, Dishinger JF, Liu CJ, et al. Induction of Ran GTP drives ciliogenesis. Molecular biology of the cell. 2011 Dec;22(23):4539-48. PubMed PMID: 21998203. Pubmed Central PMCID: 3226473.

72. Marshall WF, Rosenbaum JL. Intraflagellar transport balances continuous turnover of outer doublet microtubules: implications for flagellar length control. The Journal of cell biology. 2001 Oct 29;155(3):405-14. PubMed PMID: 11684707. Pubmed Central PMCID: 2150833.

73. Huber C, Cormier-Daire V. Ciliary disorder of the skeleton. American journal of medical genetics Part C, Seminars in medical genetics. 2012 Aug 15;160C(3):165-74. PubMed PMID: 22791528. 
74. Davenport JR, Yoder BK. An incredible decade for the primary cilium: a look at a onceforgotten organelle. American journal of physiology Renal physiology. 2005 Dec;289(6):F1159-69. PubMed PMID: 16275743.

75. Cano DA, Sekine S, Hebrok M. Primary cilia deletion in pancreatic epithelial cells results in cyst formation and pancreatitis. Gastroenterology. 2006 Dec;131(6):185669. PubMed PMID: 17123526.

76. Lehman JM, Laag E, Michaud EJ, Yoder BK. An essential role for dermal primary cilia in hair follicle morphogenesis. The Journal of investigative dermatology. 2009 Feb;129(2):438-48. PubMed PMID: 18987668. Pubmed Central PMCID: 2677658.

77. Hong SB, Oh H, Valera VA, Baba M, Schmidt LS, Linehan WM. Inactivation of the FLCN tumor suppressor gene induces TFE3 transcriptional activity by increasing its nuclear localization. PloS one. 2010;5(12):e15793. PubMed PMID: 21209915. Pubmed Central PMCID: 3012117.

78. Betschinger J, Nichols J, Dietmann S, Corrin PD, Paddison PJ, Smith A. Exit from pluripotency is gated by intracellular redistribution of the bHLH transcription factor Tfe3. Cell. 2013 Apr 11;153(2):335-47. PubMed PMID: 23582324. Pubmed Central PMCID: 3661979.

79. Bird SL, Heald R, Weis K. RanGTP and CLASP1 cooperate to position the mitotic spindle. Molecular biology of the cell. 2013 Aug;24(16):2506-14. PubMed PMID: 23783028. Pubmed Central PMCID: 3744954.

80. Burdick D, Le Gall AH, Rodriguez-Boulan E. Vesicular transport: implications for cell polarity. Biocell : official journal of the Sociedades Latinoamericanas de Microscopia Electronica et al. 1996 Dec;20(3):343-53. PubMed PMID: 9031604.

81. Ao X, Zou L, Wu Y. Regulation of autophagy by the Rab GTPase network. Cell death and differentiation. 2014 Mar;21(3):348-58. PubMed PMID: 24440914. Pubmed Central PMCID: 3921601.

82. Liang J, Mills GB. AMPK: a contextual oncogene or tumor suppressor? Cancer research. 2013 May 15;73(10):2929-35. PubMed PMID: 23644529. Pubmed Central PMCID: 3725287.

83. Behrends C, Sowa ME, Gygi SP, Harper JW. Network organization of the human autophagy system. Nature. 2010 Jul 1;466(7302):68-76. PubMed PMID: 20562859. Pubmed Central PMCID: 2901998.

84. Park H, Tsang M, Iritani BM, Bevan MJ. Metabolic regulator Fnip1 is crucial for iNKT lymphocyte development. Proceedings of the National Academy of Sciences of the United States of America. 2014 May 13;111(19):7066-71. PubMed PMID: 24785297. Pubmed Central PMCID: 4024901.

85. Park H, Staehling K, Tsang M, Appleby MW, Brunkow ME, Margineantu D, et al. Disruption of Fnip1 reveals a metabolic checkpoint controlling B lymphocyte development. Immunity. 2012 May 25;36(5):769-81. PubMed PMID: 22608497. Pubmed Central PMCID: 3361584.

86. Baba M, Keller JR, Sun HW, Resch W, Kuchen S, Suh HC, et al. The folliculin-FNIP1 pathway deleted in human Birt-Hogg-Dube syndrome is required for murine B-cell development. Blood. 2012 Aug 9;120(6):1254-61. PubMed PMID: 22709692. Pubmed Central PMCID: 3418720.

87. Zhang D, Iyer LM, He F, Aravind L. Discovery of Novel DENN Proteins: Implications for the Evolution of Eukaryotic Intracellular Membrane Structures and Human Disease. Frontiers in genetics. 2012;3:283. PubMed PMID: 23248642. Pubmed Central PMCID: 3521125.

88. Guertin DA, Sabatini DM. Defining the role of mTOR in cancer. Cancer cell. 2007 Jul;12(1):9-22. PubMed PMID: 17613433. 


\section{CHAPTER 5}

89. Hartung T, Daston G. Are in vitro tests suitable for regulatory use? Toxicological sciences : an official journal of the Society of Toxicology. 2009 Oct;111(2):233-7. PubMed PMID: 19617452.

90. Cash TP, Gruber JJ, Hartman TR, Henske EP, Simon MC. Loss of the Birt-Hogg-Dube tumor suppressor results in apoptotic resistance due to aberrant TGFbeta-mediated transcription. Oncogene. 2011 Jun 2;30(22):2534-46. PubMed PMID: 21258407. Pubmed Central PMCID: 3109270.

91. Ferrante MI, Romio L, Castro S, Collins JE, Goulding DA, Stemple DL, et al. Convergent extension movements and ciliary function are mediated by ofd 1 , a zebrafish orthologue of the human oral-facial-digital type 1 syndrome gene. Human molecular genetics. 2009 Jan 15;18(2):289-303. PubMed PMID: 18971206. Pubmed Central PMCID: 2638777.

92. van Rooijen E, Giles RH, Voest EE, van Rooijen C, Schulte-Merker S, van Eeden FJ. LRRC50, a conserved ciliary protein implicated in polycystic kidney disease. Journal of the American Society of Nephrology : JASN. 2008 Jun;19(6):1128-38. PubMed PMID: 18385425. Pubmed Central PMCID: 2396934.

93. Tobin JL, Beales PL. Restoration of renal function in zebrafish models of ciliopathies. Pediatric nephrology. 2008 Nov;23(11):2095-9. PubMed PMID: 18604564.

94. Simms RJ, Hynes AM, Eley L, Inglis D, Chaudhry B, Dawe HR, et al. Modelling a ciliopathy: Ahi1 knockdown in model systems reveals an essential role in brain, retinal, and renal development. Cellular and molecular life sciences : CMLS. 2012 Mar;69(6):993-1009. PubMed PMID: 21959375.

95. Sullivan-Brown J, Schottenfeld J, Okabe N, Hostetter CL, Serluca FC, Thiberge SY, et al. Zebrafish mutations affecting cilia motility share similar cystic phenotypes and suggest a mechanism of cyst formation that differs from pkd2 morphants. Developmental biology. 2008 Feb 15;314(2):261-75. PubMed PMID: 18178183. Pubmed Central PMCID: 2453220.

96. Zhou W, Dai J, Attanasio M, Hildebrandt F. Nephrocystin-3 is required for ciliary function in zebrafish embryos. American journal of physiology Renal physiology. 2010 Jul;299(1):F55-62. PubMed PMID: 20462968. Pubmed Central PMCID: 2904175.

97. Austin-Tse C, Halbritter J, Zariwala MA, Gilberti RM, Gee HY, Hellman N, et al. Zebrafish Ciliopathy Screen Plus Human Mutational Analysis Identifies C21orf59 and CCDC65 Defects as Causing Primary Ciliary Dyskinesia. American journal of human genetics. 2013 Oct 3;93(4):672-86. PubMed PMID: 24094744. Pubmed Central PMCID: 3791264.

98. Pathak N, Obara T, Mangos S, Liu Y, Drummond IA. The zebrafish fleer gene encodes an essential regulator of cilia tubulin polyglutamylation. Molecular biology of the cell. 2007 Nov;18(11):4353-64. PubMed PMID: 17761526. Pubmed Central PMCID: 2043541.

99. Neugebauer JM, Amack JD, Peterson AG, Bisgrove BW, Yost HJ. FGF signalling during embryo development regulates cilia length in diverse epithelia. Nature. 2009 Apr 2;458(7238):651-4. PubMed PMID: 19242413.

100. Jaffe KM, Thiberge SY, Bisher ME, Burdine RD. Imaging cilia in zebrafish. Methods Cell Biol.97:415-35. PubMed PMID: 20719283.

101. Zhou W, Dai J, Attanasio M, Hildebrandt F. Nephrocystin-3 is required for ciliary function in zebrafish embryos. Am J Physiol Renal Physiol. Jul;299(1):F55-62. PubMed PMID: 20462968.

102. Kramer-Zucker AG, Olale F, Haycraft CJ, Yoder BK, Schier AF, Drummond IA. Ciliadriven fluid flow in the zebrafish pronephros, brain and Kupffer's vesicle is required for normal organogenesis. Development. 2005 Apr;132(8):1907-21. PubMed PMID: 15790966. 
103. Bedell VM, Westcot SE, Ekker SC. Lessons from morpholino-based screening in zebrafish. Briefings in functional genomics. 2011 Jul;10(4):181-8. PubMed PMID: 21746693. Pubmed Central PMCID: 3144740.

104. Robu ME, Larson JD, Nasevicius A, Beiraghi S, Brenner C, Farber SA, et al. p53 activation by knockdown technologies. PLoS genetics. 2007 May 25;3(5):e78. PubMed PMID: 17530925. Pubmed Central PMCID: 1877875.

105. Gerety SS, Wilkinson DG. Morpholino artifacts provide pitfalls and reveal a novel role for pro-apoptotic genes in hindbrain boundary development. Developmental biology. 2011 Feb 15;350(2):279-89. PubMed PMID: 21145318. Pubmed Central PMCID: 3111810.

106. Eisen JS, Smith JC. Controlling morpholino experiments: don't stop making antisense. Development. 2008 May;135(10):1735-43. PubMed PMID: 18403413.

107. Amatruda JF, Patton EE. Genetic models of cancer in zebrafish. International review of cell and molecular biology. 2008;271:1-34. PubMed PMID: 19081540.

108. Stemple DL. TILLING--a high-throughput harvest for functional genomics. Nature reviews Genetics. 2004 Feb;5(2):145-50. PubMed PMID: 14726927.

109. Sheehan-Rooney K, Swartz ME, Zhao F, Liu D, Eberhart JK. Ahsa1 and Hsp90 activity confers more severe craniofacial phenotypes in a zebrafish model of hypoparathyroidism, sensorineural deafness and renal dysplasia (HDR). Disease models \& mechanisms. 2013 Sep;6(5):1285-91. PubMed PMID: 23720234. Pubmed Central PMCID: 3759348.

110. Sanders LH, Whitlock KE. Phenotype of the zebrafish masterblind (mbl) mutant is dependent on genetic background. Developmental dynamics : an official publication of the American Association of Anatomists. 2003 Jun;227(2):291-300. PubMed PMID: 12761856.

111. Vignet C, Begout ML, Pean S, Lyphout L, Leguay D, Cousin X. Systematic screening of behavioral responses in two zebrafish strains. Zebrafish. 2013 Sep;10(3):365-75. PubMed PMID: 23738739.

112. Dlugos CA, Rabin RA. Ethanol effects on three strains of zebrafish: model system for genetic investigations. Pharmacology, biochemistry, and behavior. 2003 Jan;74(2):471-80. PubMed PMID: 12479969.

113. Bretaud S, MacRaild S, Ingham PW, Bandmann O. The influence of the zebrafish genetic background on Parkinson's disease-related aspects. Zebrafish. 2011 Sep;8(3):103-8. PubMed PMID: 21745139.

114. Howard MT, Malik N, Anderson CB, Voskuil JL, Atkins JF, Gibbons RJ. Attenuation of an amino-terminal premature stop codon mutation in the ATRX gene by an alternative mode of translational initiation. Journal of medical genetics. 2004 Dec;41(12):951-6. PubMed PMID: 15591283. Pubmed Central PMCID: 1735636.

115. Bertram G, Innes S, Minella O, Richardson J, Stansfield I. Endless possibilities: translation termination and stop codon recognition. Microbiology. 2001 Feb;147(Pt 2):255-69. PubMed PMID: 11158343.

116. Kaler SG, Tang J, Donsante A, Kaneski CR. Translational read-through of a nonsense mutation in ATP7A impacts treatment outcome in Menkes disease. Annals of neurology. 2009 Jan;65(1):108-13. PubMed PMID: 19194885. Pubmed Central PMCID: 2917729.

117. Fearon K, McClendon V, Bonetti B, Bedwell DM. Premature translation termination mutations are efficiently suppressed in a highly conserved region of yeast Ste6p, a member of the ATP-binding cassette (ABC) transporter family. The Journal of biological chemistry. 1994 Jul 8;269(27):17802-8. PubMed PMID: 7517933.

118. Cartegni L, Chew SL, Krainer AR. Listening to silence and understanding nonsense: exonic mutations that affect splicing. Nature reviews Genetics. 2002 Apr;3(4):285-98. PubMed PMID: 11967553. 


\section{CHAPTER 5}

119. Heppner Goss K, Trzepacz C, Tuohy TM, Groden J. Attenuated APC alleles produce functional protein from internal translation initiation. Proceedings of the National Academy of Sciences of the United States of America. 2002 Jun 11;99(12):8161-6. PubMed PMID: 12034871. Pubmed Central PMCID: 123038.

120. Kozak M. At least six nucleotides preceding the AUG initiator codon enhance translation in mammalian cells. Journal of molecular biology. 1987 Aug 20;196(4):94750. PubMed PMID: 3681984.

121. Gaj T, Gersbach CA, Barbas CF, 3rd. ZFN, TALEN, and CRISPR/Cas-based methods for genome engineering. Trends in biotechnology. 2013 Jul;31(7):397-405. PubMed PMID: 23664777. Pubmed Central PMCID: 3694601.

122. Wiedenheft B, Sternberg SH, Doudna JA. RNA-guided genetic silencing systems in bacteria and archaea. Nature. 2012 Feb 16;482(7385):331-8. PubMed PMID: 22337052.

123. Auer TO, Duroure K, De Cian A, Concordet JP, Del Bene F. Highly efficient CRISPR/Cas9-mediated knock-in in zebrafish by homology-independent DNA repair. Genome research. 2014 Jan;24(1):142-53. PubMed PMID: 24179142. Pubmed Central PMCID: 3875856.

124. Hwang WY, Fu Y, Reyon D, Maeder ML, Kaini P, Sander JD, et al. Heritable and precise zebrafish genome editing using a CRISPR-Cas system. PloS one. 2013;8(7):e68708. PubMed PMID: 23874735. Pubmed Central PMCID: 3706373.

125. Jao LE, Wente SR, Chen W. Efficient multiplex biallelic zebrafish genome editing using a CRISPR nuclease system. Proceedings of the National Academy of Sciences of the United States of America. 2013 Aug 20;110(34):13904-9. PubMed PMID: 23918387. Pubmed Central PMCID: 3752207.

126. Pemberton TJ, Choi S, Mayer JA, Li FY, Gokey N, Svaren J, et al. A mutation in the canine gene encoding folliculin-interacting protein 2 (FNIP2) associated with a unique disruption in spinal cord myelination. Glia. 2014 Jan;62(1):39-51. PubMed PMID: 24272703.

127. Zon LI, Peterson RT. In vivo drug discovery in the zebrafish. Nat Rev Drug Discov. 2005 Jan;4(1):35-44. PubMed PMID: WOS:000226153200016. English.

128. Baraban SC, Dinday MT, Hortopan GA. Drug screening in Scn1a zebrafish mutant identifies clemizole as a potential Dravet syndrome treatment. Nature communications. 2013;4:2410. PubMed PMID: 24002024. Pubmed Central PMCID: 3891590.

129. Kawahara G, Karpf JA, Myers JA, Alexander MS, Guyon JR, Kunkel LM. Drug screening in a zebrafish model of Duchenne muscular dystrophy. Proceedings of the National Academy of Sciences of the United States of America. 2011 Mar 29;108(13):5331-6. PubMed PMID: 21402949. Pubmed Central PMCID: 3069215.

130. Ferlay J, Shin HR, Bray F, Forman D, Mathers C, Parkin DM. Estimates of worldwide burden of cancer in 2008: GLOBOCAN 2008. International journal of cancer Journal international du cancer. 2010 Dec 15;127(12):2893-917. PubMed PMID: 21351269.

131. Tobin JL, Beales PL. The nonmotile ciliopathies. Genet Med. 2009 Jun;11(6):386-402. PubMed PMID: 19421068.

132. Mortele KJ, Ros PR. Cystic focal liver lesions in the adult: differential CT and MR imaging features. Radiographics : a review publication of the Radiological Society of North America, Inc. 2001 Jul-Aug;21(4):895-910. PubMed PMID: 11452064.

133. Horton KM, Bluemke DA, Hruban RH, Soyer P, Fishman EK. CT and MR imaging of benign hepatic and biliary tumors. Radiographics : a review publication of the Radiological Society of North America, Inc. 1999 Mar-Apr;19(2):431-51. PubMed PMID: 10194789. 


\section{CHAPTER 6}

\section{Valorisation}


CHAPTER 6 


\section{Valorisation}

The aim of the current thesis is to gain more insight into the molecular functioning of folliculin (FLCN), the protein mutated in Birt-Hogg-Dubé syndrome (BHD), and FLCN's binding partner FNIP1 in order to better understand pathophysiology of BHD and related disorders. BHD is an inherited cancer syndrome, resulting in an increased risk on benign facial tumours called fibrofolliculomas, systemic cyst formation, pneumothorax and renal cancer. BHD is a rare disorder; worldwide approximately 200 families have been clinically diagnosed as such (1-4), which immediately raises the question why the scientific community should spend valuable time and money on research that could potentially benefit only few patients. Or in general: what is the value of investigating rare disorders?

\section{The value of investigating rare disorders}

Over the past few years there has been a change in research interest, away from rare, monogenic diseases towards more common multifactorial disease (5). This is partially caused by publication bias. As cloning and gene mapping are considered to be easier, these studies are less likely to be published in top journals. In addition, funding agencies are more inclined to support the study of common disease over rare orphan diseases. Development of orphan drugs to treat diseases as rare as Birt-Hogg-Dubé syndrome is economically unfeasible (6). However, there are several reasons to justify investigations of rare disorders.

Although rare disorders may affect relatively few people on their own, together they greatly impact society. Approximately 7000 rare disease (defined by the European Union as affecting fewer than 50 people per 100,000 population or by the Food and Drug Administration as affecting fewer than 200,000 people in the United States) are registered on Orphanet, the European rare disease portal. These diseases together affect about $7 \%$ of the population (7). Four out of five are genetic in origin or have a genetic component (7). Approximately half of these disorders are diagnosed during childhood, affecting the patients and their families for decades. This demonstrates an important argument for studying rare disease: for the sake of the affected patients.

Patients with rare disorders often face more difficult challenges than patients with more common health problems (7). Diagnosis of a rare disease is often not very straightforward, which can be frustrating for both patients and physicians (7). As doctors do not frequently encounter particular cases of rare disease they are less likely to recognise them. In addition, most rare diseases currently have no therapy that cures or treats the disease itself, as is the case for BHD (7). Additionally, patients can be faced with very personal and intimate decisions about marriage and childbearing, in particular when carrying a familial rare disorder.

For BHD patients, these issues are no different and the current dissertation contributes to solving these problems in several ways. Diagnosis of BHD may prove 
to be difficult as there is a large variability of phenotypic expression. Skin lesions are absent in approximately $20 \%$ of patients or may not be recognised by the untrained eye (4). In patients presenting with pneumothorax only the diagnosis BHD may also be missed (8). The current work contributes to increase the awareness of BHD, which is probably underdiagnosed due to these issues.

Currently there are no BHD-specific treatment options available. However, there is encouraging progress since the first description of BHD in 1975 (9) and the identification of the causative gene in 2002 (10). During the past ten years a growing body of evidence was published on the putative function of FLCN, to which the data in Chapter 2 has contributed. Together, these data increase our understanding of BHD aetiology and possibly lead to identification of therapeutic targets. The zebrafish models for Flcn and Fnip1 deficiency characterised in Chapter 3 and 4 may be of particular value. Zebrafish are very well suited for large drug screens, as they generate large numbers of offspring which can be exposed to drugs be merely adding them to the water (11). In addition, detection of phenotypes is easy due to ex vivo development and can be automated (11). Useful readouts for drug screening may be developed in the future, so that patients may benefit even if complete understanding of FLCN is lacking.

Because of the current lack of BHD-specific therapy and the increased risk on renal cancer, lifetime annual MRI screening for individuals with BHD and those at risk for the syndrome was suggested from the age of 20 years (4). These screening programs aid in the early detection and thereby management of renal cancer. Nevertheless, patients are exposed to lifelong uncertainty as only $20-30 \%$ of individuals affected with BHD will develop kidney cancer and its currently unclear which particular patients are at risk (1-3). Clarification of the function of FLCN may illuminate genotype-phenotype relations and might make it possible to predict what patients have increased risk on which clinical manifestation of BHD. The ultimate goal is to provide individual risk profiles for patients.

By pinpointing the causative gene of a disease, genetic counselling for patients carrying hereditary disease can be improved as patients can be better informed about their own risks and that of their potential offspring. The research in Chapter 4 of the current dissertation adds to resolving these issues, by identifying a mutation in the FNIP1 gene as the cause of familial multiple discoid fibromas (FMDF). Patients with FMDF develop skin lesions similar to the BHD fibrofolliculomas. However, current knowledge indicates that these patients do not have any systemic phenotype. Therefore, a simple, non-invasive mutation analysis can be used to exclude these patients from the increased renal cancer risk and from the accompanying screening program.

Thus, these studies aid in the diagnosis, development of therapy and genetic counselling for rare disorders, considerably improving quality of life of for affected patients. In addition, the group of people benefitting from research into rare disorder is rarely limited to affected patients, as insight into the pathophysiology 
of rare diseases can generate knowledge applicable to more common cellular processes.

\section{Understanding BHD pathogenesis may provide insight in development of sporadic renal cancer}

The classic argument promoting research on rare, monogenic disorders is that investigation of genetics and pathogenic mechanism of rare disease can generate insights into the causes and potential therapeutic targets of more common phenotypes. One classic example is Wilms' tumour, a rare form of renal cancer that occurs in approximately 1 in 10,000 children under the age of 15 worldwide (12). Studies of Wilms' tumour have greatly contributed to our understanding of genetics, epigenetics and molecular biology of childhood cancer and cancers in general (13). Another example is Fanconi's anemia, a rare disorder of chromosomal instability, with an incidence of 1 per 350,000 births. Fanconi's anemia research has illuminated general mechanisms of bone marrow failure, cancer pathogenesis and resistance to chemotherapy (14). In addition, studies of rare, monogenic disorders have revealed existence of genetic phenomena such parental imprinting, epistatic interaction and modifier genes (5). Together, these few examples show how investigation of rare disease can greatly contribute to our understanding of the aetiology of more common traits and to our understanding of cell biology and genetics in general.

Several clinical manifestations associated with BHD are also frequently detected in the general population, such as renal cancer. Kidney tumours account for approximately $2 \%$ of all adult malignancies (15) and kidney cancer is the ninth most common cancer in developed countries (16). 85\% of these are cancers of the renal parenchyma; renal cell carcinomas (16). The remaining $15 \%$ are cancers of the renal pelvis (17). Worldwide approximately 150,000 cases of renal cell carcinoma are diagnosed annually, leading to 78,000 cancer deaths (18). Incidence and mortality rates are almost twice as high for men as for women (15) and kidney cancer frequencies have been increasing during the past three decades (19), partially due to improved screening and detection but also due to increased incidence (18).

Epidemiological studies identified cigarette smoking, obesity and hypertension as risk factors for the development of renal cell carcinoma (17). Renal cell carcinoma is not a single entity but a heterogeneous group of tumours that arise from the renal tubule epithelium (18). The majority of renal cell carcinomas are of the clear cell $(75 \%)$ or papillary (10\%) histological subtypes, which are thought to arise from proximal convoluted renal tubule (20). Chromophobe renal cell carcinoma accounts for approximately $5 \%$ of renal cell carcinomas and arises from the intercalating cells of renal collecting duct (20). The remaining cases are either oncocytoma (4\%) or collecting duct carcinomas $(<1 \%)(17)$. 


\section{CHAPTER 6}

Hormonal and chemotherapy are not optimal for renal cell carcinoma due to low response rates (21). Therefore, current treatment options for renal cell carcinoma are (partial) nephrectomy or systemic treatment with immunotherapy, such as IL-2 and IFN $\alpha(22,23)$. However, these cytokine based treatments do not work for all patients and are not always effective. Thus, there is a need for the development of novel targeted therapies, which interfere with specific target molecules required for carcinogenesis and cancer cell growth. For these therapies to be generated we need to understand the pathogenesis of renal cancer at the cellular level. As development of sporadic renal cell carcinoma is the result of exposure to a combination of risk factors, determining the changes in cellular physiology leading to sporadic tumours is challenging.

Although most cases of renal cell carcinoma are sporadic, about $3 \%$ of cases are familial (18), caused by inherited, monogenic disorders predisposing to renal cell carcinoma development. The causative familial cancer genes are also frequently somatically inactivated in sporadic tumours, indicating that familial and sporadic tumours have similar pathogenesis. This particular subgroup of inherited renal cancers can therefore provide important insight into the molecular pathogenesis of sporadic renal cell carcinoma. The most common form of familial renal cell carcinoma is Von Hippel-Lindau disease (VHL) caused by mutations in the VHL gene, leading to a $70 \%$ lifetime risk on clear cell renal cell carcinoma (18). Unravelling the pathogenesis of VHL through functioning of the VHL protein has led to discovery of novel therapeutics such as receptor tyrosine kinase inhibitors targeting the VHL/HIF/VEGF pathways, for example sunitinib and sorafenib, or histone deacetylase inhibitors affecting gene expression. These targeted therapies are also applicable to treat sporadic renal cell carcinoma (24).

In contrast to other inherited kidney cancer syndromes, which predispose to one particular type of kidney cancer, the renal carcinomas in BHD are of various histological subtypes, including chromophobe, oncocytoma, clear cell, papillary and oncocytic hybrid (25). The molecular mechanisms associated with various histopathological subtypes of renal cell carcinoma can differ. Thus, in comparison to other familial renal carcinoma syndromes, BHD might be more representative for tumour formation in the general population.

Similar to VHL, FLCN mutations causative of BHD have also been found in sporadic renal cell carcinoma. Gad et al. reported FLCN mutations in a large series of sporadic chromophobe renal cell carcinoma (26). Although Nagy et al. did not detect any mutations in FLCN in chromophobe renal cell carcinomas or oncocytomas, they do report allelic loss of chromosome 17 including the FLCN locus in 8 out of 8 chromophobe tumours (27). Loss of one FLCN copy was also reported for $83 \%$ papillary and $25 \%$ clear cell renal cell carcinomas (28). In addition, in tumours lacking somatic mutations, FLCN might be inactivated through promoter methylation, which has been reported in $28 \%$ of sporadic kidney 
tumours, including renal oncocytomas, chromophobe, papillary and clear cell renal cell carcinomas (28). Together, these data show that somatic mutation of the FLCN gene in sporadic renal cancer is rare, but inactivation through chromosomal loss and/or promoter methylation seems to be involved in the entire spectrum of histological subtypes of renal tumours, suggesting a major role for FLCN in kidney cancer tumourigenesis. Therefore, examination of the cellular mechanisms involved in the development of renal tumours in BHD is bound to contribute to our understanding of the development of sporadic renal cancer, a major health problem in developed countries.

\section{The value of identification of BHD as a ciliopathy}

BHD patients are not only predisposed to renal cancer, but also to systemic cyst development in organs including kidney, liver, lungs and pancreas. Similar systemic cyst development is seen in a large of group of disorders, the so-called ciliopathies, which are caused by malfunctioning of the primary cilium (6). Although individual ciliopathies are rare, several hallmark features of ciliopathies are common in the general population. Kidney cysts, which also develop in BHD patients, are present in approximately 1 in 500 adults (6). Liver cysts are detected in $2-5 \%$ of the general population $(29,30)$ and pancreatic cysts have a prevalence of $2.4-13.5 \%$ in patients without known pancreatic disease (31). Examination of the ciliopathies may therefore provide unique insights into more common medical problems.

In Chapter 2 and 4 of this dissertation we report that BHD is a novel ciliopathy, that FLCN and one of its interactors, FNIP1, localise to primary cilia and associated structures and that changes in the levels of FLCN affect ciliogenesis and ciliary signal transduction. Preclinical studies show that inactivation of Kif3a results in loss of cilia and leads to cyst development in pancreas (32) and kidney (33). Therefore, we are the first to provide a cellular mechanism affected by FLCN deficiency that might explain key aspects of the BHD phenotype. This knowledge is valuable not only to increase our understanding of the function of FLCN and how the BHD phenotype arises, but also connects the BHD research field to a much larger scientific community investigating these disease caused by ciliary dysfunction. The BHD research could therefore benefit from the link between FLCN and cilia, also considering the efforts to investigate targeted therapy for ciliopathies (6). Likewise, the ciliopathy field may gain from identification of FLCN and FNIP1 as ciliary proteins.

In conclusion, it is the author's view that rare disorders deserve considerable attention from the scientific community. Firstly, for the sake of the affected patients and their families. Secondly, because investigating rare diseases will lead to a better understanding of the pathophysiology of more common disease and potentially to identification of novel therapeutic targets. Several clinical 
CHAPTER 6

manifestations associated with BHD are frequently detected in the general population, such as renal cancer and cyst formation in organs including the lungs, liver, pancreas and kidney. As FLCN is vital for cellular physiology, elucidating its functions will provide biological insights that are certain to profoundly affect other field of inquiry. 


\section{References}

1. Schmidt LS, Nickerson ML, Warren MB, Glenn GM, Toro JR, Merino MJ, et al. Germline BHD-mutation spectrum and phenotype analysis of a large cohort of families with BirtHogg-Dube syndrome. American journal of human genetics. 2005 Jun;76(6):1023-33. PubMed PMID: 15852235. Pubmed Central PMCID: 1196440.

2. Toro JR, Wei MH, Glenn GM, Weinreich M, Toure O, Vocke C, et al. BHD mutations, clinical and molecular genetic investigations of Birt-Hogg-Dube syndrome: a new series of 50 families and a review of published reports. Journal of medical genetics. 2008 Jun;45(6):321-31. PubMed PMID: 18234728. Pubmed Central PMCID: 2564862.

3. Houweling AC, Gijezen LM, Jonker MA, van Doorn MB, Oldenburg RA, van SpaendonckZwarts KY, et al. Renal cancer and pneumothorax risk in Birt-Hogg-Dube syndrome; an analysis of 115 FLCN mutation carriers from 35 BHD families. British journal of cancer. 2011 Dec 6;105(12):1912-9. PubMed PMID: 22146830. Pubmed Central PMCID: 3251884.

4. Menko FH, van Steensel MA, Giraud S, Friis-Hansen L, Richard S, Ungari S, et al. BirtHogg-Dube syndrome: diagnosis and management. The lancet oncology. 2009 Dec;10(12):1199-206. PubMed PMID: 19959076.

5. Antonarakis SE, Beckmann JS. Mendelian disorders deserve more attention. Nature reviews Genetics. 2006 Apr;7(4):277-82. PubMed PMID: 16534515.

6. Tobin JL, Beales PL. The nonmotile ciliopathies. Genet Med. 2009 Jun;11(6):386-402. PubMed PMID: 19421068.

7. In: Field MJ, Boat TF, editors. Rare Diseases and Orphan Products: Accelerating Research and Development. The National Academies Collection: Reports funded by National Institutes of Health. Washington (DC)2010.

8. Menko FH, Johannesma PC, van Moorselaar RJ, Reinhard R, van Waesberghe JH, Thunnissen E, et al. A de novo FLCN mutation in a patient with spontaneous pneumothorax and renal cancer; a clinical and molecular evaluation. Familial cancer. 2013 Sep;12(3):373-9. PubMed PMID: 23264078.

9. Hornstein OP, Knickenberg M. Perifollicular fibromatosis cutis with polyps of the colon--a cutaneo-intestinal syndrome sui generis. Archives for dermatological research Archiv fur dermatologische Forschung. 1975 Sep 12;253(2):161-75. PubMed PMID: 1200700.

10. Nickerson ML, Warren MB, Toro JR, Matrosova V, Glenn G, Turner ML, et al. Mutations in a novel gene lead to kidney tumors, lung wall defects, and benign tumors of the hair follicle in patients with the Birt-Hogg-Dube syndrome. Cancer cell. 2002 Aug;2(2):15764. PubMed PMID: 12204536.

11. Zon LI, Peterson RT. In vivo drug discovery in the zebrafish. Nat Rev Drug Discov. 2005 Jan;4(1):35-44. PubMed PMID: WOS:000226153200016. English.

12. Breslow N, Olshan A, Beckwith JB, Green DM. Epidemiology of Wilms tumor. Medical and pediatric oncology. 1993;21(3):172-81. PubMed PMID: 7680412.

13. Feinberg AP, Williams BR. Wilms' tumor as a model for cancer biology. Methods in molecular biology. 2003;222:239-48. PubMed PMID: 12710691.

14. D'Andrea AD. Susceptibility pathways in Fanconi's anemia and breast cancer. The New England journal of medicine. 2010 May 20;362(20):1909-19. PubMed PMID: 20484397. Pubmed Central PMCID: 3069698.

15. Ferlay J, Shin HR, Bray F, Forman D, Mathers C, Parkin DM. Estimates of worldwide burden of cancer in 2008: GLOBOCAN 2008. International journal of cancer Journal international du cancer. 2010 Dec 15;127(12):2893-917. PubMed PMID: 21351269.

16. Parkin DM, Muir CS. Cancer Incidence in Five Continents. Comparability and quality of data. IARC scientific publications. 1992 (120):45-173. PubMed PMID: 1284606. 
17. Lipworth L, Tarone RE, McLaughlin JK. The epidemiology of renal cell carcinoma. The Journal of urology. 2006 Dec;176(6 Pt 1):2353-8. PubMed PMID: 17085101.

18. Maher ER. Genetics of familial renal cancers. Nephron Experimental nephrology. 2011;118(1):e21-6. PubMed PMID: 21071978.

19. Mathew A, Devesa SS, Fraumeni JF, Jr., Chow WH. Global increases in kidney cancer incidence, 1973-1992. European journal of cancer prevention : the official journal of the European Cancer Prevention Organisation. 2002 Apr;11(2):171-8. PubMed PMID: 11984136.

20. Kovacs G, Akhtar M, Beckwith BJ, Bugert P, Cooper CS, Delahunt B, et al. The Heidelberg classification of renal cell tumours. The Journal of pathology. 1997 Oct;183(2):131-3. PubMed PMID: 9390023.

21. Yagoda A, Abi-Rached B, Petrylak D. Chemotherapy for advanced renal-cell carcinoma: 1983-1993. Seminars in oncology. 1995 Feb;22(1):42-60. PubMed PMID: 7855619.

22. Bukowski RM. Natural history and therapy of metastatic renal cell carcinoma: the role of interleukin-2. Cancer. 1997 Oct 1;80(7):1198-220. PubMed PMID: 9317170.

23. Bukowski RM, Novick AC. Clinical practice guidelines: renal cell carcinoma. Cleveland Clinic journal of medicine. 1997;64 Suppl 1:SI1-44; quiz SI5-7. PubMed PMID: 9239949.

24. Baldewijns MM, van Vlodrop IJ, Vermeulen PB, Soetekouw PM, van Engeland M, de Bruine AP. VHL and HIF signalling in renal cell carcinogenesis. The Journal of pathology. 2010 Jun;221(2):125-38. PubMed PMID: 20225241.

25. Vocke CD, Yang Y, Pavlovich CP, Schmidt LS, Nickerson ML, Torres-Cabala CA, et al. High frequency of somatic frameshift BHD gene mutations in Birt-Hogg-Dubeassociated renal tumors. Journal of the National Cancer Institute. 2005 Jun 15;97(12):931-5. PubMed PMID: 15956655.

26. Gad S, Lefevre SH, Khoo SK, Giraud S, Vieillefond A, Vasiliu V, et al. Mutations in BHD and TP53 genes, but not in HNF1beta gene, in a large series of sporadic chromophobe renal cell carcinoma. British journal of cancer. 2007 Jan 29;96(2):336-40. PubMed PMID: 17133269. Pubmed Central PMCID: 2360004.

27. Nagy A, Zoubakov D, Stupar Z, Kovacs G. Lack of mutation of the folliculin gene in sporadic chromophobe renal cell carcinoma and renal oncocytoma. International journal of cancer Journal international du cancer. 2004 Apr 10;109(3):472-5. PubMed PMID: 14961590.

28. Khoo SK, Kahnoski K, Sugimura J, Petillo D, Chen J, Shockley K, et al. Inactivation of BHD in sporadic renal tumors. Cancer research. 2003 Aug 1;63(15):4583-7. PubMed PMID: 12907635.

29. Mortele KJ, Ros PR. Cystic focal liver lesions in the adult: differential CT and MR imaging features. Radiographics : a review publication of the Radiological Society of North America, Inc. 2001 Jul-Aug;21(4):895-910. PubMed PMID: 11452064.

30. Horton KM, Bluemke DA, Hruban RH, Soyer P, Fishman EK. CT and MR imaging of benign hepatic and biliary tumors. Radiographics : a review publication of the Radiological Society of North America, Inc. 1999 Mar-Apr;19(2):431-51. PubMed PMID: 10194789.

31. van Asselt SJ, de Vries EG, van Dullemen HM, Brouwers AH, Walenkamp AM, Giles RH, et al. Pancreatic cyst development: insights from von Hippel-Lindau disease. Cilia. 2013;2(1):3. PubMed PMID: 23384121. Pubmed Central PMCID: 3579754.

32. Cano DA, Sekine S, Hebrok M. Primary cilia deletion in pancreatic epithelial cells results in cyst formation and pancreatitis. Gastroenterology. 2006 Dec;131(6):185669. PubMed PMID: 17123526. 
33. Patel V, Li L, Cobo-Stark P, Shao X, Somlo S, Lin F, et al. Acute kidney injury and aberrant planar cell polarity induce cyst formation in mice lacking renal cilia. Human molecular genetics. 2008 Jun 1;17(11):1578-90. PubMed PMID: 18263895. Pubmed Central PMCID: 3150596. 



\section{CHAPTER 7}

\section{Summary}

Samenvatting

\section{Acknowledgements / Dankwoord \\ Curriculum vitae}


CHAPTER 7 


\section{Summary}

Birt-Hogg-Dubé syndrome (BHD; OMIM \#135150) is an autosomal dominant disorder predisposing to benign facial tumours called fibrofolliculomas, lung cysts, pneumothorax and renal cancer. BHD is a rare disorder; approximately 200 families have been diagnosed worldwide. The syndrome is caused by mutations in the FLCN gene, most of which truncate its protein product, folliculin (FLCN). Current therapeutic options for BHD patients are limited and can only treat symptoms, not the underlying cause. To develop BHD-specific treatment and strategies to prevent renal cancers from developing, it is crucial to unravel BHD pathophysiology and FLCN's function in cellular physiology.

FLCN is highly conserved throughout evolution, suggesting an important biological role. The protein is thought to act as a tumour suppressor and has been implicated in the regulation of various signalling pathways and cellular processes. However, how FLCN mutations cause cellular defects that lead to the BHD phenotype remains to be determined.

An emerging aspect of the BHD phenotype is cyst formation in organs including the lungs, liver, pancreas and kidney. Development of cystic organs is one of the hallmarks of a large group of disorders, the so-called ciliopathies, caused by malfunctioning of the primary cilium. This "cellular antenna" projects from the apical cell surface of non-proliferating epithelial cells. It is involved in chemo- and mechanosensation and provides transduction of various signalling pathways, including the Hedgehog pathway, the canonical Wnt pathway and the noncanonical Wnt pathway under control of planar cell polarity (PCP). The latter is of special interest, as PCP regulates oriented cell division in the kidney, leading to lengthening of the kidney tubules without changes in tubular diameter. Disruption of PCP causes cyst formation. As the BHD phenotype is clinically similar to the ciliopathies, we investigate the role of FLCN in the primary cilium in Chapter 2. We show that FLCN localises to primary and motile cilia and associated structures. Alteration of FLCN levels causes changes in ciliogenesis, polarised growth of kidney cells and canonical Wht signalling. Expression of FLCN-K508R mutant protein does not affect ciliogenesis in monolayer and only mildly affects polarised growth, suggesting that the mutant protein has lost a large part of its functionality. Together these data show that BHD is a novel ciliopathy caused by malfunctioning of the primary cilium. As defective ciliogenesis has been reported to result in cyst formation, these data explain how loss of FLCN leads to a cystic phenotype in BHD patients.

Determining protein interaction may give an insight into the cellular functions of a protein of interest. Thus, novel interactors for FLCN were identified, including folliculin interacting protein 1 (FNIP1). In vitro, FNIP1 was shown to support the 


\section{CHAPTER 7}

role of FLCN in various pathways, suggesting that FLCN and FNIP1 have a similar function.

It is therefore surprising that humans with a FNIP1 mutation only have a relatively mild skin phenotype, described in Chapter $\mathbf{4}$ as familial multiple discoid fibromas (FMDF; OMIM \%190340). We identify a heterozygous mutation in FNIP1 in FMDF patients and show that the mutant FNIP1 mRNA is broken down by nonsense-mediated decay, suggesting that FMDF is caused by FNIP1 haploinsufficiency. FMDF patients develop skin lesions resembling those found in BHD but they have different histopathology, are often localized on the ears and are of childhood onset, while the BHD lesions typically appear after the age of 25 . Moreover, we found no evidence for pulmonary or renal complications, which could be due to tissue-specific compensation by FNIP2 or functional redundancy between FNIP1 and FNIP2. Alternatively, loss of a single FNIP1 allele might simply be tolerated in these tissues, possibly as a result of higher baseline FNIP1 levels compared to skin.

Although we show that FNIP1 localises to the primary cilium, similar to FLCN, our data presently do not support ciliary dysfunction as a major contributing factor to FMDF development. Thus, the cellular mechanism that causes FMDF remains to be determined.

In vitro studies have certain limitations, primarily because cultured cells are maintained in an artificial environment in which interactions between cells and tissues cannot be mimicked. Therefore, we aimed to study the function of FLCN and FNIP1 in an animal model. Studies using experimental animals have shown that FLCN is required for embryonic development, but the exact developmental role of the protein is difficult to examine in available animal models as homozygous FLCN knockout causes embryonic lethality in mammals. Mice with homozygous knockout of Fnip1 are viable, although development of particular immune cell subsets is affected. However, how this translates to the FMDF lesions is currently unclear. Thus, we sought a different animal model that would allow us to examine the role of FLCN and FNIP in development.

Zebrafish (Danio rerio) are very well suited to study the pathophysiology of both developmental defects and carcinogenesis, because of their ex vivo development enabling easy visualisation and manipulation, their large offspring production and their similar tumour formation to humans. In addition, many ciliopathies have been modelled in zebrafish and these models show similar phenotypes. Therefore, we characterise zebrafish loss of function models for Flcn and Fnip1 in Chapter $\mathbf{3}$ and $\mathbf{4}$ in order to learn more about the function of these proteins.

Zebrafish flcn and fnip1 have similar expression patterns throughout development as determined by whole mount in situ hybridization. Both genes are expressed in the eye, fin bud, somites and regions of the brain. Zebrafish Flcn and 
Fnip1 loss of function models show that both proteins are required for zebrafish embryogenesis, as their deficiency causes a severe developmental phenotype characterised by brain oedema, problems with tail circulation and a larger yolk and thinner yolk extension in 2-day old morphants. Although part of these morphant phenotypes is reminiscent of ciliopathy zebrafish models, we did not detect any obvious defects in the appearance of cilia in either pronephros or central canal after extensive whole mount immunofluorescent studies using acetylated alpha tubulin antibodies in the flcn and fnip1 morphants. However, the normal appearance does not rule out the possibility that the cilia that were detected in the morphants might not be functional.

Alternatively, these phenotypes may be caused by cell cycle defects. In zFucci transgenic embryos, the G1 phase of the cell cycle is fluorescently labelled red and S, G2 and M are labelled green. zFucci flcn morphants have a decreased amount of cells in S-M phase and a corresponding increase in G1 cells, particularly in the retina and compartments of the brain including the tectum. It remains to be determined if Flcn directly regulates the cell cycle in these embryos or whether these defects are secondary, possibly resulting from metabolic defect caused by Flcn deficiency.

Although its exact function is not completely understood, it has become clear that FLCN is vital for cellular physiology. FLCN regulates ciliogenesis, orientation of the mitotic spindle and canonical Wnt signalling. Malfunctioning or loss of primary cilia has been reported to drive cyst formation, explaining how loss of FLCN leads to a cystic phenotype in BHD patients. However, it remains to be determined how loss of FLCN leads to cancer. Recent studies on the crystal structure of FLCN's carboxyl-terminus and on FLCN's role in regulating lysosomal GTPases suggest that FLCN may be a major regulator of vesicle transport through a GAP and/or GEF function towards small GTPases of the Ras superfamily, including RhoA, Rab and Ran. This hypothesis would not only explain how FLCN affects ciliogenesis, but also why FLCN deficiency has such widespread cellular consequences. Further research is required to investigate this hypothesis and unravel the cellular role of FLCN and its interactors, including FNIP1. However, even if complete understanding of the function of FLCN and FNIP1 is lacking, potential therapeutic targets may be identified, using the novel zebrafish loss of function models described in this thesis. In addition, investigation of BHD pathophysiology may provide a unique opportunity to study the aetiology of clinical manifestations common in the general population, such as renal cyst formation and renal cell carcinoma. As FLCN is an ancient and crucial component of the cellular machinery, elucidating its functions will provide biological insights that are certain to profoundly affect other field of inquiry. 


\section{Samenvatting}

Birt-Hogg-Dubé syndroom (BHD) is een erfelijke ziekte, vernoemd naar de drie Canadese artsen Arthur Birt, Georgina Hogg en William Dubé. BHD is zeldzaam; wereldwijd zijn er slechts 200 families bekend met dit syndroom. Tachtig procent van de BHD patiënten ontwikkelt goedaardige tumoren in de vorm van huidkleurige bultjes op het gezicht, de nek en het bovenlichaam. Daarnaast heeft $80 \%$ van de patiënten longcysten, die kunnen leiden tot klaplongen. Het meest bedreigende voor BHD patiënten is echter een verhoogd risico van $20-30 \%$ op nierkanker.

BHD wordt veroorzaakt door veranderingen in het DNA, zogenoemde mutaties, van het FLCN gen. Een gen is een functioneel stuk DNA waar een eiwit van gemaakt wordt. Bijna alle cellen van het menselijk lichaam bevatten twee kopieën van elk gen, één kopie afkomstig van de moeder en één van de vader. BHD erft dominant over, wat inhoudt dat mensen de ziekte ontwikkelen zodra ze van hun vader óf van hun moeder een gemuteerde kopie krijgen. Bij de geboorte is dan in alle cellen van het lichaam één gemuteerde en één normale FLCN kopie aanwezig. Niet alle BHD families hebben dezelfde mutatie in het FLCN gen. Ongeveer 100 verschillende BHD-veroorzakende mutaties zijn beschreven. Van het FLCN gen wordt het eiwit folliculine (FLCN) gemaakt en de meeste mutaties leiden tot de productie van een verkort FLCN eiwit.

De behandeling van BHD patiënten bestaat momenteel uit symptoombestrijding. De huidtumoren en nierkanker kunnen operatief worden verwijderd, maar er is een grote kans dat ze terugkomen. Om specifieke behandelingen te ontwikkelen of om mogelijk de symptomen te kunnen voorkomen, is het van groot belang dat we weten wat FLCN precies doet en hoe mutaties in FLCN BHD veroorzaken.

Door eiwitten van verschillende diersoorten digitaal met elkaar te vergelijken hebben we gezien dat FLCN in de loop van de evolutionaire ontwikkeling behouden is gebleven, wat suggereert dat het een belangrijke functie heeft. Omdat in de niertumoren van BHD patiënten zowel de FLCN kopie van de moeder als van de vader een mutatie heeft, wordt aangenomen dat FLCN onder normale omstandigheden tumorvorming blokkeert (i.e. tumor suppressor). Wanneer gedurende het leven van BHD patiënten de gezonde FLCN kopie ook gemuteerd raakt, valt deze blokkering weg en kunnen tumoren ontstaan. Hoe deze blokkering precies werkt binnen cellen en waarom hij wegvalt als FLCN gemuteerd raakt proberen we te ontrafelen met het onderzoek in dit proefschrift.

In Hoofdstuk 2 van dit proefschrift wordt beschreven dat BHD patiënten niet alleen in hun longen cysten ontwikkelen, maar ook in andere organen waaronder de lever, de alvleesklier en de nieren. Een soortgelijke cystenvorming wordt gezien bij een grote groep andere ziekten bekend als de ciliopathiën, die allemaal door hetzelfde mechanisme veroorzaakt worden. Bij de ciliopathiën werken de trilharen 
(cilia) niet meer goed. Bijna alle cellen van het menselijk lichaam hebben één trilhaar, een soort cellulaire antenne, die vanaf het celmembraan uitsteekt naar de omgeving van de cel. Deze trilhaar stelt de cel in staat omgevingssignalen op te vangen en hierop te reageren door signalering in de cel aan te passen. Studies met muizen hebben aangetoond dat wanneer deze trilharen niet meer goed werken cysten kunnen ontstaan. Omdat BHD patiënten ook cysten krijgen, is in Hoofdstuk $\mathbf{2}$ onderzocht of FLCN een rol speelt bij het functioneren van deze trilharen.

Dit onderzoek heeft aangetoond dat FLCN aanwezig is in verschillende soorten trilharen. Daarnaast hebben we minder trilharen gedetecteerd op cellen waarin FLCN kunstmatig verlaagd of verhoogd is. Om te bestuderen of FLCN een rol speelt in het functioneren van de trilharen, gebruiken we een model om de communicatie tussen cellen en hun omgeving te simuleren. Daarvoor worden niercellen in collageen gekweekt. De cellen vormen dan sferoïden, balvormige structuren met in het midden een holle ruimte waar trilharen in steken. Manipulatie van FLCN in deze kweken leidt tot kleinere sferoïden met minder trilharen en kleinere holtes, waar nog cellen in zitten. Het inbrengen van mutant FLCN in deze systemen heeft geen effect op de vorming van trilharen en sferoïden, wat suggereert dat het mutante eiwit niet functioneel is.

Deze data laten zien dat BHD een nieuwe ciliopathie is, die wordt veroorzaakt door verstoring van de trilharen. Omdat eerder onderzoek heeft aangetoond dat door defecte trilharen cysten kunnen ontstaan, kan dit verklaren waarom BHD patiënten cysten krijgen.

Een alternatieve manier om de functie van het eiwit FLCN the achterhalen is te bepalen aan welke andere eiwitten FLCN bindt. Wanneer van deze zogenoemde bindingspartners de functie bekend is, kan dit mogelijk informatie geven over wat FLCN doet. De afgelopen jaren zijn verschillende bindingspartners van FLCN geïdentificeerd, waaronder folliculin interacting protein 1 (FNIP1). In experimenten met celkweek lijkt het alsof FNIP1 zich hetzelfde gedraagt als FLCN.

Het is daarom verrassend dat mensen met een mutatie in het FNIP1 gen alleen maar huidtumoren krijgen, zoals beschreven in Hoofdstuk 4. In een aantal families met familiaire multipele discoïde fibromen (FMDF), die allen van één voorouder af blijken te stammen, hebben we een mutatie in één kopie van het FNIP1 gen gevonden. Deze mutatie zorgt ervoor dat productie van het eiwit wordt verminderd. De patiënten krijgen dan huidtumoren die op het eerste gezicht lijken op de BHD tumoren. Echter, onder de microscoop zien deze tumoren er anders uit. Een ander onderscheid kan gemaakt worden in het moment van ontstaan. Mensen met FMDF ontwikkelen huidtumoren al tijdens de kindertijd, terwijl de BHD huidtumoren na het 35ste levensjaar ontstaan. Daarnaast hebben FMDF patiënten, voor zover bekend, geen afwijkingen aan de longen of nieren. Hieruit blijkt dat FLCN en FNIP1 in mensen niet dezelfde functie hebben. Mogelijk is FNIP1 niet zo 


\section{CHAPTER 7}

belangrijk in de longen en nieren, of kan verlies ervan worden opgevangen door andere eiwitten.

Net als FLCN bevindt FNIP1 zich ook in de trilharen, maar kunstmatige vermindering van FNIP1 in cellen leidt niet tot een verandering van het aantal trilharen. Hoe verminderde productie van FNIP1 leidt tot ontwikkeling van de huidtumoren is nog niet bekend.

Wetenschappelijk onderzoek met cellen (in vitro) heeft enkele beperkingen. Cellen worden onder kunstmatige omstandigheden gekweekt en de interactie tussen verschillende weefsels kan niet worden bekeken. Daarom is het nuttig FLCN en FNIP1 in een diermodel (in vivo) te bestuderen.

FLCN is belangrijk voor de embryonale ontwikkeling van zoogdieren. Muizen, ratten en honden waarbij de beide kopieën van het FLCN gen een mutatie hebben, sterven tijdens de ontwikkeling. Het is moeilijk te achterhalen waarom dit precies gebeurt, omdat de embryo's zich dan nog in de baarmoeder bevinden. Muizen waarbij beide kopieën van het FNIP1 gen gemuteerd zijn worden wel geboren, maar hebben een verstoring in bepaalde cellen van het afweersysteem. Hoe zich dit vertaalt naar FMDF bij mensen is onduidelijk.

Zebravissen zijn zeer geschikt voor onderzoek naar zowel de embryonale ontwikkeling als naar kanker. Tumorvorming in vissen lijkt op die in mensen. Daarnaast produceren zebravissen grote aantallen nakomelingen. De moeder zet de eieren af in het water (zoals kikkerdril) waarna de vader ze bevrucht. Doordat ze buiten het lichaam van de moeder groeien, is de ontwikkeling van zebravis embryo's gemakkelijk onder een microscoop te bekijken en te manipuleren. De embryo's ontwikkelen zich razendsnel; binnen twee dagen zijn de voorlopers van de belangrijkste organen al te zien. Daarnaast zijn voor een aantal van de ciliopathiën zebravismodellen gemaakt, die op elkaar lijken. Daarom worden in Hoofdstuk 3 en 4 zebravis modellen voor het verlies van FLCN en FNIP1 gekarakteriseerd.

Eerst hebben we bepaald of en waar in het zebravis embryo Flcn en Fnip1 aangemaakt worden. Tijdens de ontwikkeling worden beide eiwitten geproduceerd in dezelfde structuren, waaronder voorlopers van de vinnen, bepaalde delen van de hersenen, het oog en de somieten (voorlopers van de wervelkolom, de ribben, een deel van de huid en de rugspieren). Daarnaast hebben we gezien dat Flcn en Fnip1 allebei nodig zijn voor de embryonale ontwikkeling van de zebravis. Verstoring van de productie van Flcn of Fnip1 in bevruchte eicellen veroorzaakt problemen in twee dagen oude embryo's, waaronder vochtophoping in de hersenen, verstoorde bloedcirculatie in de staart en een vergrootte dooierzak. Een deel van deze problemen wordt ook gezien bij zebravismodellen voor ciliopathiën, maar er werden geen afwijkingen in de trilharen van deze embryos gedetecteerd. Mogelijk functioneren de trilharen niet goed, ondanks dat ze er normaal uit zien. 
Het zou ook kunnen zijn dat de verstoring van embryonale ontwikkeling als gevolg van verminderd Flcn of Fnip1 het resultaat is van een verstoorde celdeling. De Fucci transgene zebravis lijn heeft kunstmatig aangepast DNA, waardoor de cellen van deze vissen in verschillende fasen van de celcyclus een andere kleur fluorescent licht uitstralen. Fucci zebravis embryos met verminderd Flcn hebben een lager aantal delende cellen in S, G2 en M fase en een verhoogd aantal nietdelende cellen in G1 fase, voornamelijk in het oog en in delen van de hersenen. Het is momenteel niet bekend of Flcn zelf de celcyclus reguleert of dat deze defecten indirect zijn doordat Flcn bijvoorbeeld het metabolisme beïnvloedt.

Wat FLCN precies doet is niet bekend, maar het is de afgelopen jaren wel duidelijk geworden dat FLCN van groot belang is voor het normaal functioneren van cellen. Zo reguleert FLCN de trilharen en vorming van sferoïden. Eerder onderzoek heeft uitgewezen dat wanneer de trilharen niet goed werken, cysten kunnen ontstaan. Dit verklaart waarom mensen met FLCN mutaties cysten krijgen, maar niet waarom mutatie van FLCN kanker veroorzaakt. Recente studies suggereren dat FLCN het transport van andere eiwitten tussen verschillende delen van de cel verzorgt. Omdat dit transport van groot belang is voor allerlei cellulaire processen, waaronder de opbouw van de trilharen, zou deze hypothese niet alleen verklaren waarom de trilharen aangedaan zijn, maar ook waarom in cellen met verminderd FLCN een groot aantal andere processen verstoord zijn. Meer onderzoek is nodig om te kijken of deze hypothese klopt en om te achterhalen wat de echte functie is van FLCN en zijn bindinsgpartners, waaronder FNIP1. Zelfs als we niet precies weten wat FLCN en FNIP1 doen is het misschien mogelijk om nieuwe behandelingen te ontwikkelen voor BHD en FMDF met behulp van de zebravismodellen beschreven in dit proefschrift. Omdat zebravissen veel nakomelingen produceren die eenvoudig zijn bloot te stellen aan geneesmiddelen door deze slechts aan hun omgevingswater toe te voegen, zijn deze modellen zeer geschikt om op grote schaal medicijnen te testen. Hierbij worden mogelijk middelen gevonden die de problemen veroorzaakt door verlaagd FLCN of FNIP1 kunnen verhelpen. Deze medicijnen kunnen wellicht gebruikt worden om BHD/FMDF patiënten te behandelen.

Ondanks dat BHD een zeldzame ziekte is, is het van groot belang dat we erachter komen hoe BHD ontstaat. Een aantal van de symptomen van BHD, zoals cystenvorming en nierkanker, komt namelijk veel vaker voor bij de algemene bevolking. Daarom zal dit onderzoek naar alle waarschijnlijkheid ook inzicht geven in deze veelvoorkomende medische problemen. Aangezien FLCN een oeroud onderdeel is van de cel, zal het onderzoeken van zijn functie ongetwijfeld inzichten opleveren die ook voor andere vakgebieden van belang zullen zijn. 
CHAPTER 7 
Acknowledgements / dankwoord 


\section{Curriculum vitae}

Monique Nicole Helena Luijten was born in Cadier en Keer, The Netherlands on November 26, 1986. She received her secondary education degree at the Porta Mosana College in Maastricht in 2005. In the same year she enrolled in Molecular Life Sciences at Maastricht University. In 2008 she obtained her bachelors degree cum laude and enrolled in the Clinical Molecular Sciences master programme at the same university. During these studies she worked as an intern under supervision of Dr. Petra Wolffs in the Department of Medical Microbiology of the Academic Hospital in Maastricht for six months on the topic of bacterial antibiotic resistance. After a second, eight month internship in the Experimental Dermatology lab with Prof. Dr. Maurice van Steensel at Maastricht University she wrote her Master thesis on the cancer-causing Birt-Hogg-Dubé syndrome. In 2010, she graduated cum laude and successfully applied for a Kootstra talent fellowship with the data from her thesis. This allowed her to continue the Birt-Hogg-Dubé research as a $\mathrm{PhD}$ candidate. A Dutch Cancer Society (KWF) travel grant for academics enabled her to work in the lab of Prof. Dr. Ferenc Müller at the University of Birmingham in the United Kingdom as part of her PhD for one year. There she worked on the development of a zebrafish model for Birt-Hogg-Dubé syndrome.

Monique Nicole Helena Luijten werd op 26 november 1986 geboren in Cadier en Keer. In 2005 behaalde zij haar VWO diploma aan het Porta Mosana College te Maastricht, waarna ze Moleculaire Levenswetenschappen ging studeren aan de Universiteit Maastricht. In 2008 behaalde ze haar bachelor diploma cum laude en begon aan de master Clinical Molecular Sciences aan dezelfde universiteit. Tijdens deze studie werkte ze zes maanden als stagiaire in het lab van Dr. Petra Wolffs van de afdeling Medische Microbiologie in het Academisch Ziekenhuis Maastricht, waar het onderzoek zich richtte op de antibioticaresistentie van bacteriën. Na een tweede stage van acht maanden in het lab Experimentele Dermatologie bij Prof. dr. Maurice van Steensel schreef ze haar master scriptie over het erfelijke tumorsyndroom van Birt-Hogg-Dubé. In 2010 studeerde ze cum laude af en met de data van deze scriptie vroeg ze met goed gevolg een Kootstra Talent Fellowship aan, waardoor ze het onderzoek naar het Birt-Hogg-Dubé syndroom kon voortzetten als promovenda. In het kader van dit promotieonderzoek liep ze met behulp van een KWF beurs een jaar stage in het lab van Prof. dr. Ferenc Müller aan Birmingham University in het Verenigd Koninkrijk waar ze werkte aan een zebravis model voor het Birt-Hogg-Dubé syndroom. 
